SEASIDE TOWN REGENERATION AND THE INTERCONNECTIONS

BETWEEN THE PHYSICAL

ENVIRONMENT, KEY AGENCIES

AND MIDDLE-LIFE MIGRATION

ANTHONY LEONARD

PHD

2014 


\section{SEASIDE TOWN REGENERATION AND THE INTERCONNECTIONS BETWEEN THE PHYSICAL ENVIRONMENT, KEY AGENCIES AND MIDDLE-LIFE MIGRATION}

\section{ANTHONY LEONARD}

A thesis submitted in partial fulfilment of the requirements of the University of Brighton for the degree of Doctor of Philosophy

July 2014 


\section{Abstract}

Seaside resorts' fortunes have changed over the past half a century, and as a consequence many of the towns' physical environments and inhabitants have altered. Many grew in population size through in-migration, particularly as a result of retirement, which took over from the holiday industry as a process that changed the socio-economic and cultural structures of these places. Bexhill-onSea, East Sussex is among those seaside towns that have undergone such changes, fuelled by exogenous forces but also influenced by key agencies actions that have been a catalyst for altering the physical environment which encouraged the in-migration of middle-life people and the retired. This thesis analyses the effect of these changes and the role key agencies have had. In particular, it argues the changing nature of retirement in-migration of 'middlelifers' (aged 50-70), those approaching or entering retirement, has had a profound effect on the town. This thesis disputes conventional retirement migration theories identifying a new form of 'lifestyle-affirming' migration.

Based on empirical findings from semi-structured interviews with key agencies and middle-lifers, as well as desktop research, the methodology adopts a Lamarckian conceptual and theoretical perspective of socio-economic and cultural evolution that focuses on the influence on socio-economic change of the physical environment, key agency actions and middle-life migration. The thesis evidences both Darwinian and Lamarckian perspectives on evolutionary socio-economic change are useful for understanding resorts but at a local level human agency is a key component able to influence the cultural 'circonstances' which allows for changes in the physical environment and adaptation of the socio-economic, cultural and human capital of the seaside town. In Bexhill-onSea the elected officials and resident community were agencies that influenced socio-economic change and evolution.

The town attracted middle-life migrants based on their personal experiences elsewhere that they imprinted on the town and from images and labels that were attached such as 'Costa Geriatrica'. Their choice of destination was constructed on 'gut-feel' from fleeting visits and perceived images that appealed to those middle-lifers who felt they would 'fit-in' with likeminded people and the town's culture. They brought their previous habitus and life experiences into an environment that is stable, quiet, safe and unchanging and best suits their social class disposition. This 'lifestyle-affirming' migration and 'personality-fit' was a significant and key finding in the research arguing for a new conceptualisation of middle-lifer and retirement migration of wanting to 'live by the sea' in a town that reflects their 'personality' and habitus, thus revealing more complicated forms of retirement or semi-retirement mobility. As middlelifers influence the nature of migration to seaside towns the consequential impact could be significant and questions the robustness of current retirement migration typologies in the light of the changing nature of retirement.

The result of these forms of migration is a clustering of likeminded people who have resisted local authority regeneration attempts and so regeneration practitioners need to understand better the interconnections between the physical environment, key agencies and middle-life people living in the destination, as well as the images and labels attached. 
Contents

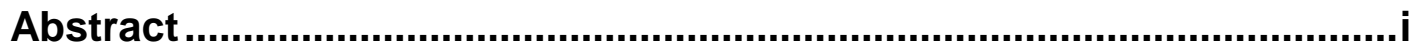

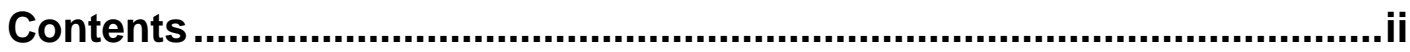

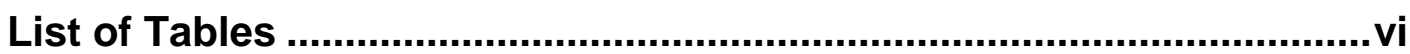

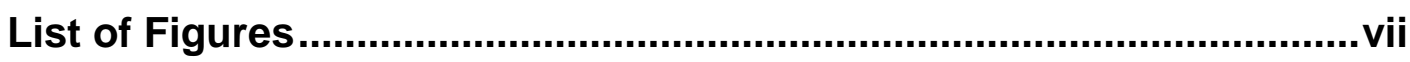

List of Appendices ..........................................................................ii

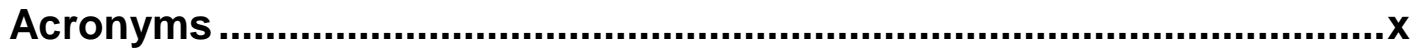

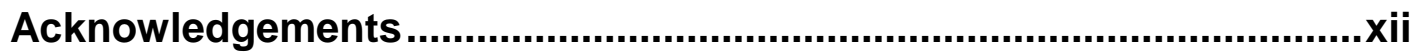

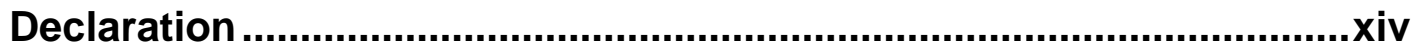

Chapter 1

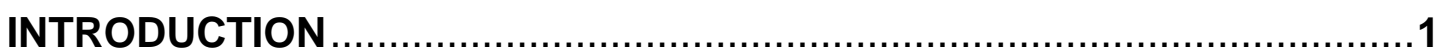

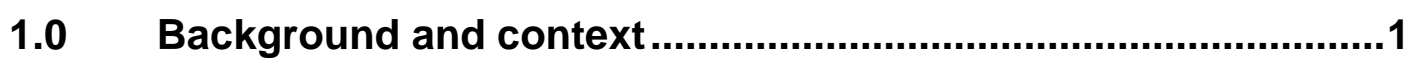

1.1 Rationale for understanding middle-life retirement migration as a process shaping seaside towns ..........................................

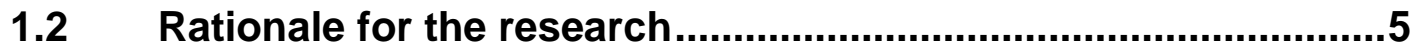

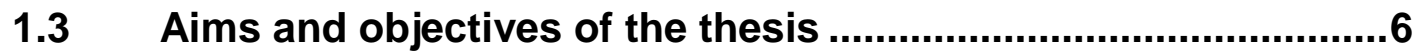

1.4 Rationale for the selection of Bexhill-on-Sea .............................

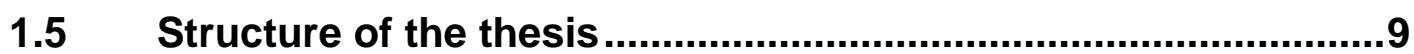

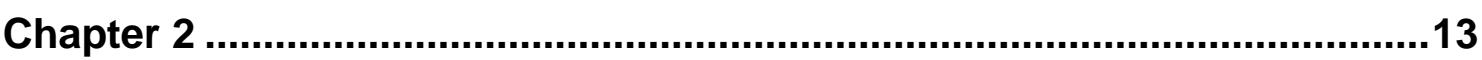

CURRENT UNDERSTANDINGS OF PRINCIPAL ENGLISH SEASIDE

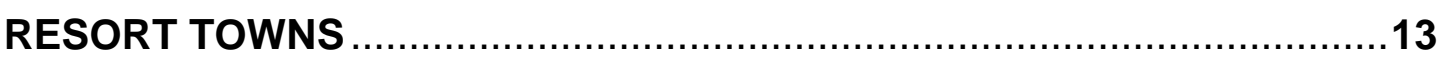

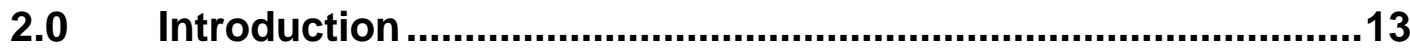

2.1 Declines in English seaside resorts ........................................15

2.2 Postmodern interpretations of seaside resort towns ...............20

2.2.1 Middle-life migration as a process shaping seaside towns .....24

2.3 Evolution and resort lifecycles: Lamarckian analysis ..............29

2.4 Regeneration and the 'New Economy' ......................................35

2.5 Key agency and enterprise-based models of analysis.............40

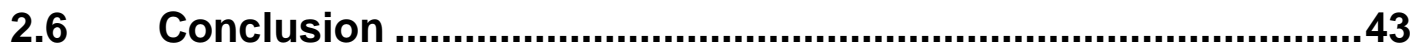

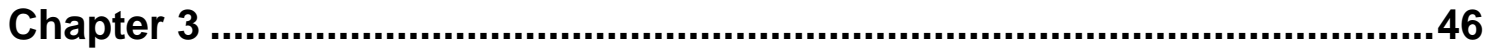

CHARACTERISTICS OF MIDDLE-LIFE MIGRATION AND SYNTHESIS

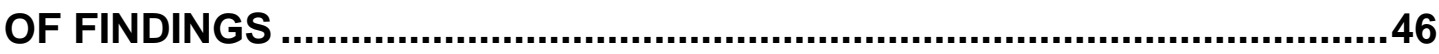

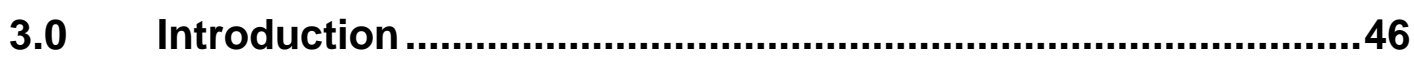

3.1 Characteristics of middle-lifers ............................................... 47 


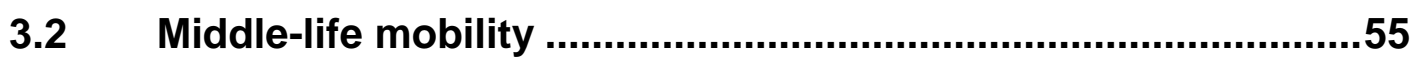

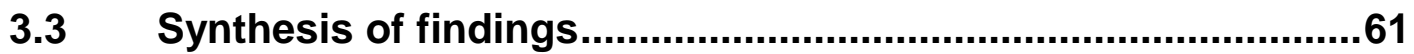

3.4 Introducing the focus of examination .......................................65

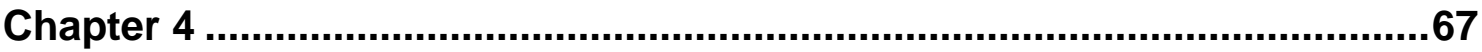

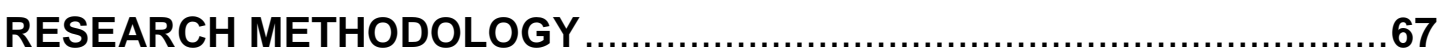

4.0 Introduction - theoretical influences and methods ..................67

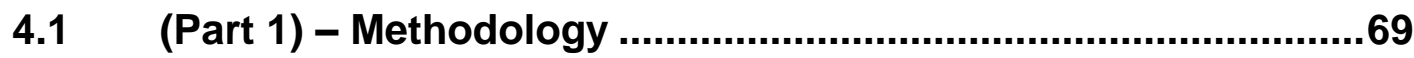

4.1.1 Theoretical and epistemological issues ..................................69

4.1.2 Research methodology and narrative inquiry ...........................73

4.1.3 The Case Study approach ..........................................................79

4.1.4 Bexhill-on-Sea as a single-case study ....................................83

4.2 (Part 2) - Research Design........................................................ 84

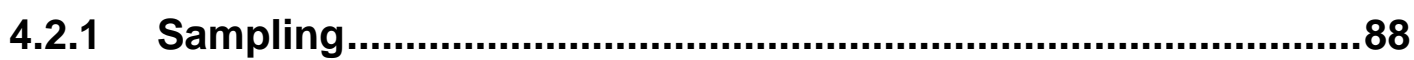

4.2.2 Interviews and questions .........................................................90

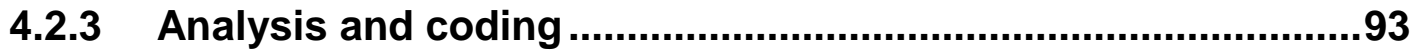

4.2.4 Validity, authenticity and credibility of the research findings .95

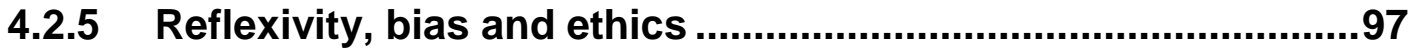

4.2.6 Self-awareness and reflexivity.............................................100

4.3 Conclusion ..........................................................................101

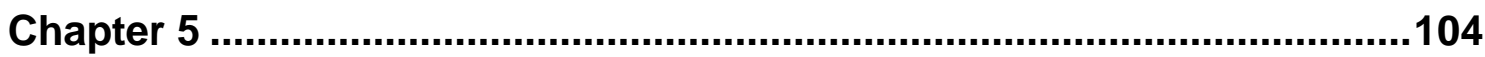

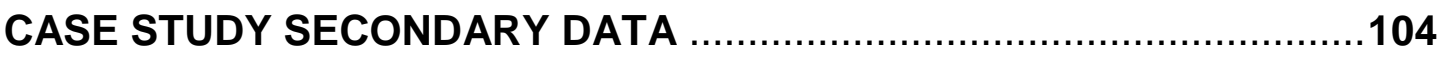

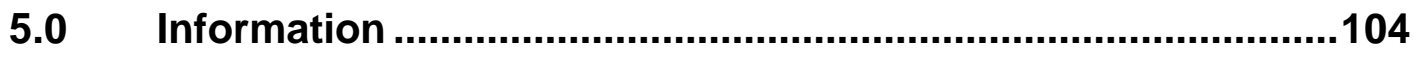

5.1 Bexhill-on-Sea profile ...........................................................104

5.2 Foundations and early development of the resort town of Bexhill-on-Sea ........................................................................107

5.3 Social divisions within the town of Bexhill-on-Sea.................109

5.4 Employment and economic status ........................................111

$5.5 \quad$ Benefit claimants ................................................................113

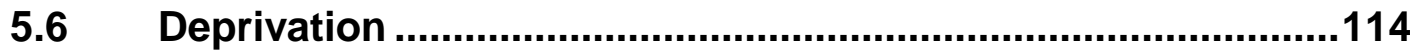

5.7 Bexhill-on-Sea indices of multiple deprivation and employment

5.8 Household migration flows: Rother District and Hastings Borough

5.9 Hastings and Bexhill-on-Sea regeneration ............................121

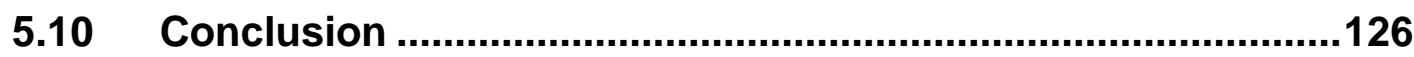


Chapter 6

ANALYSIS - PHYSICAL ENVIRONMENT AND AGENCY

6.0 Introduction

6.1 Participants' perceptions of Bexhill-on-Sea's environment...127

6.2 Perceptions of economic decline and performance 134

6.2.1 The perceptions of Bexhill-on-Sea 135

6.2.2 Migration and employment 137

6.2.3 The local economy. 141

6.3 Perceptions of the governance of Bexhill-on-Sea 143

6.3.1 Governance and urban planning

6.4 Perceptions of Bexhill-on-Sea's future socio-economic performance 151

6.5 Perceptions of the role of local leadership............................155

6.6 Conclusion - progress with research findings .......................159

Chapter 7 162

ANALYSIS - IN-MIGRATION OF MIDDLE-LIFE PEOPLE TO BEXHILL-ON-SEA 162

7.0 Introduction 162

7.1 Participant profiles 162

7.2 Middle-life in-migration motivations ....................................164

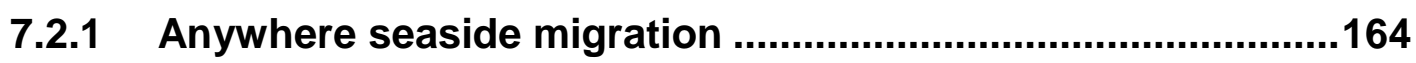

7.2.2 Counter-urbanisation and the 'seaside idyll'..........................166

7.3 Practicalities and typologies of middle-life migration ............168

7.4 'Personality' migration ...........................................................173

7.5 Image and place identity ......................................................177

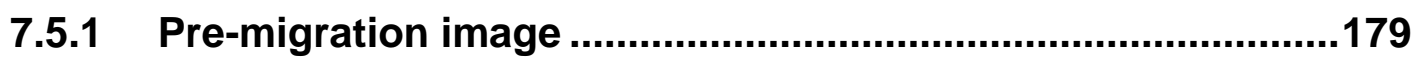

7.5.2 Post migration - positives ..................................................180

7.5.3 Post-migration - negatives and 'personality' migration

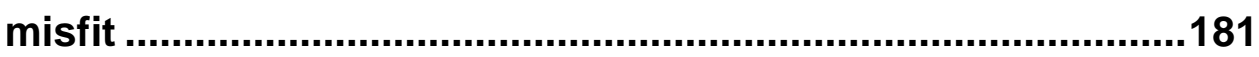

7.6 Regeneration, employment and image .................................184

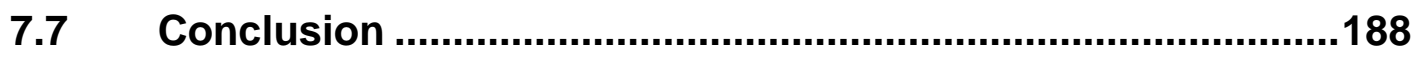

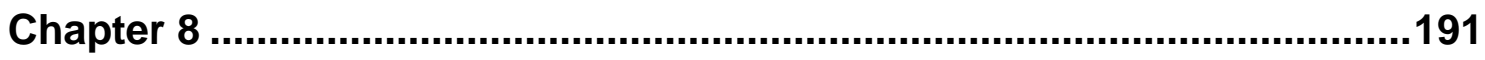

SYNTHESIS OF FINDINGS AND CONCLUSION ...................................191

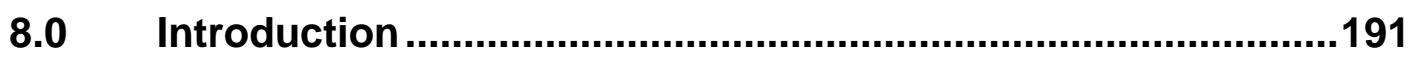

8.1 Resort structure evolution and tourism area lifecycle ...........193 
8.1.1 Resort evolution - Darwinian and Lamarckian key findings..194

8.2 Interconnections between structure and agency...................197

8.2.1 Role of key agency governance and leadership .....................202

8.2.2 Structure and agency key findings .......................................206

8.3 Perceptions, images and labels - contributory findings.........208

8.4 Middle-life migration to Bexhill-on-Sea - significant and key

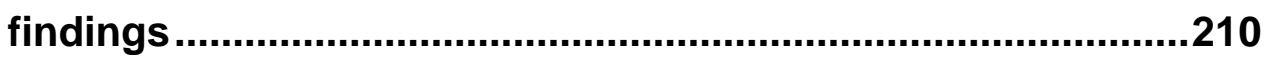

8.4.1 'Lifestyle-affirming' migration and 'personality fit' within

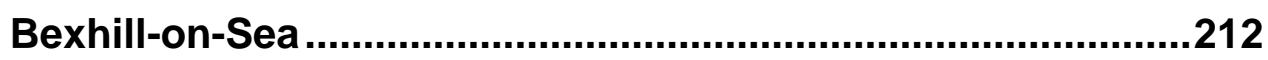

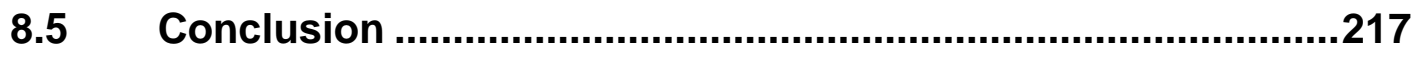

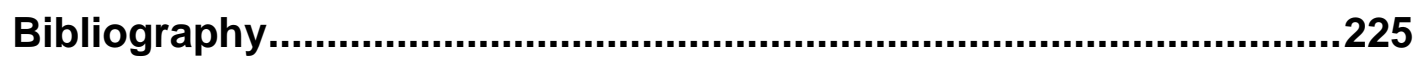

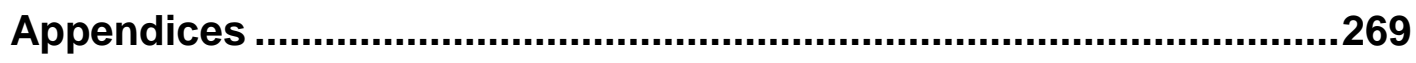




\section{List of Tables}

Table Title

Page

Chapter 3 Characteristics of Middle-Life Migration and Synthesis of Findings

Table $3.1 \quad$ Time spent in different life stages.

\section{Chapter 4 Research Methodology}

Table 4.1 List of principal key agencies researched and key actors interviewed.

Table 4.2 List of middle-life migrants to Bexhill-on-Sea in the past five years.

Table 4.3 Secondary analyses of documentary sources.

\section{Chapter 5 Case Study Secondary Data}

Table 5.1 Bexhill-on-Sea populations by age group, 2010.

Table 5.2 Population by age group - mid-term estimates 2010.

Table 5.3 Labour market accounts for total working age population, 112 English seaside towns 1971-2001.

Table 5.4 Average deprivation ranking - LSOA's in England's principal seaside towns, 2007.

Table 5.5 Indices of multiple deprivation at LSOA for Bexhill-on-Sea. 116

Table 5.6 Migration by age group in Rother district, 2001-2011. 120

Table 5.7 Dependency ratios for East Sussex.

\section{Chapter 7 Analysis - In-Migration of Middle-Life People to} Bexhill-on-Sea

Table 7.1 Participant profiles 


\section{List of Figures}

$\begin{array}{lll}\text { Figure Title } & \text { Page }\end{array}$

Chapter 2 Current Understandings of Principal English Seaside Resort Towns

Figure 2.1 England's 37 principal seaside resort towns.

Chapter 4 Research Methodology

Figure 4.1 Analytical processes.

Chapter 5 Case Study Secondary Data

Figure 5.1 Map of southeast England.

Figure 5.2 Hastings and Rother household migration flows; year preceding 2001 census. 


\section{List of Appendices}

$\begin{array}{lll}\text { Appendix Title } & \text { Page }\end{array}$

Appendix 1 Satisfaction ratings for English local authorities' - 269 categories for coastal towns' classification (2003).

Appendix 2 English local authorities identified categories for coastal 270 towns and their life expectancy rates against the English Average, 2003.

Appendix 3 Population mid-term estimates and age distribution for the principal England's seaside towns, 2006

Appendix 4 Vickers et al., classification of local authority areas, 2003.

Appendix 5 Net migrants and net migration rates by district classification, 2000-01.

Appendix 6 Net migrants and net migration rates by district classification - broad age groups, 2000-01.

Appendix 7 Top and bottom 10 in/out-migration rates between pairs of Vickers et al., classification groups, by broad age group.

Appendix 8 Employment by sector in England's principal seaside towns, 2006, (ranked by public administration).

Appendix 9 A conceptual model of elderly migration and community change.

Appendix 10 Net in-migration of working age into seaside towns by age band, 1971-1991 and labour market accounts for total working age population, seaside towns 1971-2001

Appendix 11 Employment and income status of middle-life participants.

Appendix 12 12a. Participant Consent Form.

12b. Meetings Information Sheet.

Appendix 13 Interview schedules for:

13a. Non-statutory agencies e.g. corporate business.

13b. Non-statutory agencies e.g. Chamber and SME.

13c. Statutory agencies.

Appendix 14 Interview schedule for middle-lifers. 
Appendix 15 University of Brighton, School of Environment and

Technology.

Ethics Approval Form MPhil/PhD and Staff Research

Projects.

Appendix 16 16a. Population projection for Bexhill-on-Sea for 2031.

16b. Population profile for Rother district, 2001 (against UK average).

16c. Population projection for England 2018 and 2031.

Appendix 17 Factors influencing English coastal resort capacity.

Appendix 18 Median hourly earnings in England's principal seaside towns, 2007 (ranked by male earnings).

Appendix 19 Employment in England's principal seaside towns, 2006.316

Appendix 20 Economic inactivity among working age residents of seaside towns, 1981-2001.

Appendix 21 Working age and pension credit claimant rates in England's principal seaside towns, May 2007 (working age ranked by total).

Appendix 22 Bexhill detailed locality data for some of the LSOAs, January 2012.

Appendix 23 Rother indices of multiple deprivation data 2010.

Appendix 24 Summary of participant career and income profiles. 


\section{Acronyms}

\begin{tabular}{|c|c|}
\hline AARP & American Association of Retired Persons \\
\hline ABG & Area Based Grants \\
\hline AGE UK & Formerly AGE Concern and Help the Aged \\
\hline AiF & Area Investment Framework \\
\hline BBC & British Broadcasting Corporation \\
\hline CABE & Commission for Architecture and the Built Environment \\
\hline CAQDAS & Computer Assisted Qualitative Data Analysis Software \\
\hline CIPFA & Chartered Institute of Public Finance and Accountability \\
\hline CIPFA/Solace & $\begin{array}{l}\text { Chartered Institute of Public Finance and } \\
\text { Accountancy/Society of Local Authority Chief Executives }\end{array}$ \\
\hline DCLG & Department for Communities and Local Government \\
\hline DCMS & Department of Culture, Media and Sport \\
\hline DFT & Department for Transport \\
\hline $\mathrm{DH}$ & Department for Health \\
\hline DLWP & De La Warr Pavilion \\
\hline DTZ & Property Consultants \\
\hline DTZ /Pieda & Property Consultants \\
\hline DWP & Department for Work and Pensions \\
\hline $\mathrm{EH}$ & English Heritage \\
\hline ELSA & English Longitudinal Study of Ageing \\
\hline ESCC & East Sussex County Council \\
\hline ESif & East Sussex in Figures (East Sussex County Council) \\
\hline ESRC & Economic \& Social Research Council \\
\hline ETB & English Tourist Board \\
\hline ETC & English Tourism Council \\
\hline EU & European Union \\
\hline GOSE & Government Office for the South East \\
\hline HBC & Hastings Borough Council \\
\hline HCA & Homes and Community Agency \\
\hline HM Government & Her Majesty's Government \\
\hline HM Treasury & Her Majesty's Treasury \\
\hline $\mathrm{HMO}$ & Houses of Multiple Occupations \\
\hline HMSO & Her Majesty's Stationery Office \\
\hline IMD & Indices of Multiple Deprivation \\
\hline Kippers & $\begin{array}{l}\text { Kids in Parents' Pockets Eroding Retirement Savings } \\
\text { (acronym) }\end{array}$ \\
\hline LEP & Local Enterprise Partnership \\
\hline LSOA & Lower Super Output Areas \\
\hline NEF & New Economic Foundation \\
\hline NPV & Net Present Valuations \\
\hline NRF & Neighbourhood Renewal Programmes \\
\hline ODPM & Office of the Deputy Prime Minister \\
\hline ONS & Office of National Statistics \\
\hline PFI & Private Finance Initiatives \\
\hline PLUREL & Peri-Urban Land Use Relationships Consultancy \\
\hline PPP & Public Private Partnerships \\
\hline RDA & Regional Development Agencies \\
\hline $\mathrm{RD}$ & Rother District Council \\
\hline & Return on Investments \\
\hline
\end{tabular}


SEEDA

SELEP

SHRM

SMTE

SRB

TALC

URBED
South East England Development Agency South East Local Enterprise Partnership Society of Human Research Management Small and Medium-size Tourism Enterprises Single Regeneration Budget Tourism Area Life Cycle Urban and Economic Development Group 


\section{Acknowledgements}

The completion of my $\mathrm{PhD}$ has been a challenging journey and I have found that life (whether work or personal) doesn't stand still and wait for you to finish it. After six years of studying and at times frustration I knew I had to complete my $\mathrm{PhD}$ on my own terms. Naturally, it would not have been completed without the support of people around me and so there are a number who I wish to acknowledge.

Firstly, I would like to thank my PhD advisors, Professor Andrew Church and Professor Neil Ravenscroft who guided me during these past six years. Both have been challenging and offered invaluable insight and support during the research, without which I would not have completed the work. Their encouragement and supervision was welcomed for which I thank them.

I would like to thank the following people at the university who were all part of my $\mathrm{PhD}$ progression at some point in the time. To Sarah Longstaff and Ursula O'Toole who are often the unsung heroes and who helped me with the pedantic matters of course administration. Also thanks go to the Brighton University PhD committee for their helpful advice and encouragement at each stage of the process.

I would like to acknowledge the participants in my research study who gave up their valuable time and who allowed me into their lives to undertake the interviews.

I would like to thank those who encouraged me to commence this long journey. There is my previous Chief Executive, Derek Stevens, who as a longstanding resident of Bexhill-on-Sea was enthused to know more about the dynamics and complexities of the town. To Professor Julian Crampton (as Chairman of the Hastings and Bexhill Regeneration company) who gave me the encouragement to embark on this challenge. To Dr Pav Ramewal who gave me the drive, support and the determination to finish. Without their initial belief in me I would not have ventured on this path.

Importantly, are my work colleagues not least Della Knott and Roma Olden, my Personal Assistants who have had to share and endure my journey of frustrations but who have kept me on track and assisted me with the technology and administrative support to complete the work. My thanks also go to Malcolm Johnston my colleague Executive Director who gave me the space and moral support to complete my work along with the office team Jane McCullough and Caroline Agombar. A special thanks to Brenda Mason who saw me through the final hurdle whilst going through changes in her own life that puts it all into perspective. Also thank you to Councillor Maynard as Leader of Rother District Council for his patience in waiting for the final document and as sponsor of the studies. 
To my friends who had to endure lectures on the subject whether at work, when out for social events or whilst surfing and in return they constantly pestered me about 'when are you going to finish this PhD'?

Finally, and most importantly, there is my family who were my bedrock; not least my parents who have always believed in me and always there to give me support; my three children, Jamie, Tristan and Tessa who during my time studying managed to complete their own degrees and apprenticeships. I am immensely proud of them. My thanks also go to James and Emma my stepchildren, who gave me the space to study. Last, but by no means least to my wife, Alison. We endured some bumpy times during this period of our lives but we have come through it stronger, united and have much to look forward to. It is now my turn to give you the support, love and attention you deserve. Your support, kindness and love finally got me through this for which I am eternally grateful.

I thank each and every one of you - now time for a proper surf trip and to spoil my wife and family! 


\section{Declaration}

I declare that the research contained in this thesis, unless otherwise formally indicated within the text, is the original work of the author. The thesis has not been previously submitted to this or any other university for a degree, and does not incorporate any material already submitted for a degree.

Signed:

Dated: 


\section{Chapter 1}

\section{INTRODUCTION}

\subsection{Background and context}

Most principal English seaside resorts have seen a decline in their fortunes over the past half a century, due to a number of factors, not least global changes in the tourism market (Shaw and Williams, 1997; Agarwal and Shaw, 2007). As a result, many of the towns' physical environments, populations and images have altered, affecting the socio-economic performance and characteristics of these places (Walton and Browne, 2010). This complex interaction between their physical environment, the people both visiting and living and the associated labels and images has altered the seaside town original structure and purpose and created problems that require detailed investigation.

Despite our historical fondness and emotion for seaside resorts, our understanding of both the causes of decline and the effective responses by those responsible for economic and social well-being is lacking. There exists a complex series of intricate and interlocking exogenous and endogenous drivers that have led to variations in principal English seaside resort towns' socioeconomic and cultural characteristics and distinctiveness (Cooper, 1997; Williams and Shaw, 1997; Urry, 1997; Walton, 2000; Agarwal, 2005) and these require further examination. Those resorts in decline have, over the past few decades, presented an image of physical and social 'deprivation' to attract government grants, which in some cases have further compounded the decline by discouraging private sector investment and those clusters of people who bring with them the innovation and creativity necessary to regenerate seaside towns. For some statutory and non-statutory agencies this is perceived as a problem, while for others it is perceived differently, in that seaside resort decline and renewal are particular problems for the local authority, current residents and local businesses (Cooper, 2006; Walton and Browne, 2010).

An evolutionary and resort lifecycle perspective can help in understanding these changes by seeing them as being the result of a complex series of exogenous 
forces and endogenous actions and interactions between the physical environment, key agency decisions, migration patterns and the image and perceptions these notions present. The interplay between structure and agency is examined, however, the decline of seaside resorts varies and so understanding these place-specific outcomes requires an understanding of local-level changes. Some resorts have used local infrastructure to their advantage by reusing buildings as new attractions, while others have seen their buildings converted to less popular and perhaps more damaging uses such as houses of multiple occupation (HMOs), retirement flats or care homes. In some seaside resort towns the impact of decline has been profound, with some of this degeneration and deprivation being attributed to the in-migration of particular groups of people, such as the elderly, which has changed the resort structure (Benedict and Houghton, 2009). Changes in the physical environment, with signifiers in the landscape such as old people's homes and retirement flats, have altered the structure of resorts, as well as the images and perceptions of seaside destinations, by inheriting descriptors such as the 'Costa Geriatrica' and 'God's Waiting Room'. As resort tourism transformed many seaside towns' grew in population through in-migration, particularly from retirement, that took over from the holiday industry that has altered the socio-economic and cultural characteristics of these destinations. With retirement a key driver of migration to seaside resorts, the actions of agencies have altered the structure of the physical environment, in order to accommodate retirement living. These actions have also altered the socio-cultural and economic vibrancy of seaside resorts and contributed to their decline, thus leading to questions regarding whether many of these towns even function as resorts. However, with an ageing Western population, increasing ill-preparedness for extended old age and retirement life course patterns and behaviours becoming progressively complex, retirement lifestyle migration requires rigorous examination in the understanding of seaside towns' socio-economic and cultural regeneration.

The structural changes and the people who visit and live in a destination influence the shaping of the social ecology and 'personality' of a place, which in turn contribute to the changing fortunes of many seaside resorts (Coles, Duval and Hall, 2004; Benedict and Houghton, 2009). By analysing the effect of these changes and the actions of key agencies that have allowed this to happen, in 
particular, the changing nature of the retirement in-migration of the 'babyboomer' generation, known throughout this thesis as 'middle-lifers' (aged 50-70) entering retirement or semi-retirement, provides new knowledge to the understanding of seaside resorts. These interrelations and interconnections between changes in the physical environment and key agency actions to support retirement in-migration and the socio-economic performance of these destinations are not currently well understood. Furthermore, they are perceived differently by varying agencies and those visiting and living in these places, which in turn may conflict with the destinations' regeneration objectives - an issue which requires detailed examination.

This thesis therefore contributes to a better understanding of retirement migration motivations and the interconnections between the physical environment and key agencies, which may assist regeneration practitioners and policymakers in improving the socio-economic performance of seaside resort towns.

\subsection{Rationale for understanding middle-life retirement migration as a process shaping seaside towns}

When middle-lifers prepare for and enter retirement, they will have a profound effect on current popular seaside retirement migration patterns. Whereas the size of retirement cohorts can be projected in advance (Keyfitz, 1982), middlelifers' motivations and decision-making in retirement are less predictable (Bailey and Livingstone, 2007) and add to the uncertainty and likely impact on current seaside retirement destinations. With retirement expected to become far more multifaceted as the traditional sharp divide between work and retirement begins to dissolve, the consequences for resorts and seaside retirement towns could be significant.

There is growing evidence that retirement migration motivations and behaviours are changing and becoming increasingly diverse, and to where middle-lifers choose to migrate as they enter a new life phase could become an important feature for future seaside economies. The over 50 s also account for half the nation's spending and three quarters of its wealth (ELSA, 2003, 2010), though 
there is evidence that shows growing diversity amongst the increasing older population - whereas many are more healthy and wealthy than ever before, some have low incomes and experience poor health (Hirsch, 2003; Jefferies, 2005). This diversity is important in the context of ageing and retirement behaviours and never more so than in English seaside towns. Whether seaside towns remain as appealing to middle-life 'semi-retirees' or 'retirees' as they have been to previous cohorts of retirees over the past five or so decades, is unclear. Life course patterns have become progressively more diverse, and the changeover into retirement is not always clear cut, which may lead to the redistribution of people in uneven ways and instigate distinct socio-spatial migration patterns. Societal predictions suggest that we are moving towards a more 'have' and 'have-not' society and a polarisation between those with wealth, good health, education and income and those who will be dependent on the welfare state for support (Scharf et al., 2002; Bailey et al., 2006). In previous years as people aged, so they were less likely to work, principally as a consequence of policies that encouraged early retirement (European Policies Research Centre, 2006). However, early retirement is becoming less common than it was in previous decades, and middle-lifers are looking to remain economically active for longer as demographic ageing takes effect (Atterton, 2006). This may have important impacts on current fashionable seaside retirement towns and their future actions.

Therefore the aim of this research is to examine socio-economic and cultural change and regeneration in a seaside town to understand the interconnections between processes of change in the physical (human-made built and urban) environment, key agencies that influence change and the effects of middle-lifer migration (aged 50-70 years of age).

The thesis considers emerging themes on middle-life migration, unravelling some of the established labels and categories attached to migrants and how individuals perceive and value destinations. The complex interaction between their prior experiences of a location, their perceptions created through wider cultural imaginings (through media labels and images), certain historical conditions and their distinct situations at the stage of migration (including economic, educational and cultural capital) are explored. Furthermore, this 
research study identifies under what theme retirement migration falls, whether it may be constrained by the reflexive habitus (Bourdieu, 1984, 1990; Sweetman, 2003) of individuals and so their class disposition, lifetime experiences, intertwined with their contemporary consumer living may take on greater meaning in future migration decisions. Therefore, while the selection of a retirement destination may be an intended choice about how to live, it may also be the result of structural constraints, individual agency and reflexive habitus. These migration motivations are closely aligned to core sociological variables such as class and ethnicity, but they become increasingly fragile as this standardisation is dismantled through consumerism and how people go about constructing their own identities and clustering with likeminded people and cultures (Coles and Timothy, 2004; Florida, 2008). By understanding the causes of retirement in-migration decisions to a seaside resort town will thereby assist future regeneration policymaking decisions.

The literature review identified that middle-lifers have had different life experiences and therefore have more diverse attitudes to those of earlier retirees. Their relationship with consumption and the physical environment is complex, and for many this will involve how they go about finding a seaside location that best suits their 'individuality' and allows them to maintain their consumer lifestyle. The thesis therefore analyses middle-lifers' reasons for migrating and their habitus and reflexivity, which are critical components of emerging middle-life, motivation-led migration.

\subsection{Rationale for the research}

The significance of exogenous forces and endogenous actions that have affected resorts' performance requires local investigation, as it appears that local responses to and interaction with broader external activity have produced 'place-specific' outcomes that influence the socio-economic and cultural characteristics of the locality. The assertion that 'place' makes a difference to a resort destination is therefore examined in this thesis. This recognition of understanding local-level change supports the need for locality studies that analyse the courses that underlie evolution and development. The concept of resort lifecycle, as presented by Butler (1980), is brought into question by an 
understanding of the processes of interaction between structure and agency and how place characteristics operate and develop over time. Martin (2006) suggests that resort destinations are not only passive victims of global forces but are also affected by the impact of local politics and subsequent decisions and actions. Whether disrupting and shaping the physical environment through physiological interventions by key agencies creates the conditions for adapting and modifying the nature of the people living in and visiting a destination to improve socio-economic conditions, is considered in the research. The research literature and an analysis thereof therefore identifies these structural changes and how certain agencies' actions are able to influence structures and the physical environment and in turn affect the demographic make-up of those both visiting and living in a seaside town and influence the shaping of the image and personality of the place, thereby altering its socio-economic characteristics.

Therefore, the ability of key agencies to improve the physical environment seems to be an important consideration. Cooper (2006) discusses how destinations adapt to suit new markets, and this thesis examines these interdependencies between the policies of key agencies that can alter the physical environment, the adaptation and migration of human social and cultural capital (in this case middle-lifers) and the images and perceptions that are created. For many seaside towns, their physical environment, as currently presented, may need to alter if they are to appeal to middle-lifers whose attitudes towards retirement change and as the need or desire to work alters.

The following summarises the aims of the thesis and the areas identified for further research through which to develop a better understanding of seaside town regeneration.

\subsection{Aims and objectives of the thesis}

There is insufficient research on interplay between key agencies, the physical environment (which includes the human-made built and urban environment) and on the motivations and behaviours of retirement migration to seaside resort towns and their impact that requires detailed examination. The aim of this research is to examine socio-economic and cultural change and regeneration in 
a seaside town to understand the interconnections between processes of change in the physical environment, key agencies that influence change and the effects of middle-lifer migration (aged 50-70 years of age). The thesis examines these issues through the case study of Bexhill-on-Sea.

The conceptual and theoretical content of the thesis relates to debates in the literature over middle-life and retirement migration taking an evolutionary perspective. In particular, the research explores not only the actions of key agencies but also the role of middle-lifer in-migrants as potential key actors shaping the town.

Furthermore, the thesis examines the conventional conceptualisation of retirement migration and identifies this as problematic by highlighting more complex forms of retirement or semi-retirement mobility. The research challenges the robustness of current retirement migration typologies to seaside towns and reveals other motives that are of equal importance, such as psychosocial fit (Erikson, 1987; Sweetman, 2003) and the changing outlook on retirement as retirement patterns become increasingly diverse. It therefore aims at developing a better understanding of middle-life migration motivations to resorts, including not only the influence of economic factors but also the effect of lifestyle patterns and aspirations that could affect their destination of choice and clustering. Related to this are the perceptions and images of a place held by middle-lifers when making migration decisions and the interconnections between the physical environment, key agencies and middle-life migration.

The thesis exposes the varied and multifarious forces at work in a seaside resort town and concludes on the leadership role and actions of key agencies on the physical environment, the image on offer and the unfolding of a more complex form of retirement in-migration. In addition, the thesis presents the dynamics at work in Bexhill-on-Sea, showing the fluidity and dynamism of socioeconomic and cultural changes. Based on empirical findings from semistructured interviews with key agencies and middle-lifers, as well as desktop research, this thesis demonstrates the role of middle-lifers, in order to refocus attention onto retirement migration and the consequential impact this may have on seaside resort retirement towns. 


\subsection{Rationale for the selection of Bexhill-on-Sea}

The southeast coast of England offers a useful setting for the case study location, as a number of seaside resort towns along this coastline display higher than average retirement populations. The research will focus on Bexhill-onSea, which statistically has one of the oldest populations and greatest longevity in England, reinforced by its label of 'Costa Geriatrica'. The transition from a traditional seaside resort to a seaside retirement town has changed the complexion of the town and the loss of its seaside tourism market share and the consequent impact on related infrastructure, image and economic performance. The once fashionable, up-market Edwardian resort, now in slow decline, follows neatly the Butler (1980) TALC empirical model. However, in recognition of this decline, the local authority is seeking to reverse the deprivation by intervening in the physical environment through regeneration projects that will adapt and attract a more varied demographic mix and thus 'improve' the town's image and socio-cultural and economic vitality. This aspect of 'complexity' exemplifies and focuses the characteristics that this thesis wishes to examine from an evolutionary and lifecycle perspective and plays a major role in the selection of units of analysis. The complexity of seaside towns as dynamic and multifarious environments, shaped by exogenous and endogenous socio-economic forces, key agency actions, retirement migrant populations and image, meant Bexhillon-Sea was considered an appropriate seaside town in the southeast of England for further investigation.

The author of this thesis has also worked for the past twenty years as a Director for the local authority that embraces Bexhill-on-Sea. This offered valuable insights and provided the 'convenience' and opportunity to overcome 'accessibility' constraints, access to information, political nuances and individuals to support the research findings that otherwise may have been more problematic. This enabled the researcher to become closely involved within the field, as this created human conditions that generated 'enough trust' for the participants to disclose their views on the subject under investigation. Also, the language used by the key agencies was already familiar to the researcher in his capacity as Executive Director, thus reducing barriers to understanding between both parties during interviews and secondary data analysis. 
While sponsored by the local authority, the work that the researcher did for this thesis was entirely independent, and Rother District Council did not influence the specific nature of the aims or objectives. To position the researcher as undertaking academic research the study was carried out in his own time and in his own surroundings. Also the methods used and the clear audit trail developed allowed the researcher to reflect on his role as a key agent and his knowledge of the case study location. The researcher's position meant that he needed to be particularly self-aware of and reflective on two particular aspects. The first was the possibility that interviewees would provide narratives that they felt the researcher wanted to hear, due to the authority and position that the researcher carried in his employment capacity. However, all the statutory agencies, and some non-statutory agencies, interviewed held senior authoritative positions, and whilst a number were known to the researcher, they were given guidance on the purpose of the interview and the role of the researcher as a research student. Therefore, dress code, language (body and verbal), presentation of the student identity card, use of university-headed paper to introduce the 'meetings information sheet' and 'consent forms' all helped to reposition the researcher in his role as a student investigator as opposed to the Executive Director of a local authority. The second aspect that required attention was that the researcher might construct his own meaning from the narratives based on his prior knowledge. This was avoided through the interviews being recorded, transcribed, coded and indexed and referenced back to field notes, as well as corroboration using secondary data.

\subsection{Structure of the thesis}

Chapter 1 provides a brief overview, history and background of seaside resort towns and retirement in-migration and sets out the problems they face and the aims and objectives of this thesis.

Chapter 2 reviews the literature on change in seaside resorts, including poststructural and postmodern perspectives, by drawing on their histories, distinctiveness, socio-economic perspectives and transitions through to their current day functions. The chapter explores the resort lifecycle using a 
Lamarckian socio-economic and cultural evolutionary analysis and by addressing the role of agency and structure. The chapter also examines the literature on urban regeneration policies affecting England's seaside towns and the socio-economic and cultural ecologies that shape a destination, which are observed from the perspective of the current recession and an evolving 'New Economy' (Florida, 2010) in the context of structure and agency. Further, it considers how the rejuvenation (Butler 2006b), abandonment, adaptation (Cooper, 1997; Baum, 1998, 2006) or co-existence (SeaSpace, 2003a, 2003b) of tourism may impact on resorts, and in particular the influence of the Lamarckian evolutionary theory in relation to the physical environment, its people and images that are created. It also looks at the physiological interventions made by various key agencies that can alter the course of seaside towns' TALC through the role of entrepreneurialism and leadership.

Chapter 3 reviews middle-life geographic mobility motivations from the point of view of previous retirement research and retirement migration typologies. It explores the concept that people, places and personalities concentrate into areas where the clustering of likeminded people can generate place personalities and the people who occupy these places fundamentally make the location through the images they create and the messages they give out.

The chapter also examines how the pension changes, working patterns, people living longer and the consumer lifestyle in which this cohort has grown up (Hirsch, 2003; Baker, 2005; European Policies Research Centre, 2006; Cetron and Davies, 2010) have all impacted retirement migration to seaside towns. However, a lifestyle migration search may be constrained by the reflexive habitus (Bourdieu, 1984, 1990; Hetherington, 1998; Sweetman 1999, 2003) of individuals, and so their class disposition within the physical environment may take on greater meaning to places such as seaside retirement towns. The chapter synthesises the preceding chapters and identifies the gap in the research, to explore a discourse of middle-life migration on local economies in seaside towns, and in particular Bexhill-on-Sea, and implications for regeneration. It also identifies that agency and structure both have influence over the demographics in seaside towns and that people shape the personality of places as well as their physical, social, economic and cultural tone. The 
research problem is discussed and addressed through a set of research aims and objectives.

Chapter 4 sets out the chosen research methodology and approach that will be used to reveal a complex set of related processes which will deliver the aims of the research. The chapter is set out in two distinct parts. Part one focuses on theoretical and epistemological influences on the choice of methodology and contains three subsections that discuss the theoretical perspectives underlining the research design, the related epistemological issues shaping the choice of research methods and discourse analysis and the choices concerning the adoption of a single-case study as part of the research design. Part two considers the details of the research design and methods used, and it also presents arguments concerning reflexivity, ethics and bias that highlight how research design ensures the validity, authenticity and credibility of the findings.

Chapter 5 examines secondary data on Bexhill-on-Sea. It presents its historical legacy and the structure of the town's socio-economic and demographic profile and examines migration movements and regeneration activity within and around the locality. While Bexhill-on-Sea is often analysed together with the neighbouring seaside resort of Hastings, the town is treated separately, as the resort's characteristics are distinctly different from Hastings.

Chapters 6 and 7 examine the research findings for the Bexhill-on-Sea case study and analyses the complex interrelations and interconnections between processes of change in the physical environment and the key agencies of change and how these key agencies perceive their attempts to influence the physical environment and socio-economic characteristics. The chapters scrutinise the town's governance (past and present) and the outcomes of these actions. It also examines the migration motivations of middle-lifers (aged 50-70 years) and their perceptions of the place and how these may best reflect their personality and the environment they seek when making migration decisions.

Chapter 8 synthesises the key arguments and addresses the knowledge gap by contextualising the over-arching problem. The chapter highlights the major contributions of the thesis and determines how its findings fulfil the original 
research aims and objectives. It also discusses how mobility flows can reshape the social and cultural ecology and structure of resorts, to support Florida's (2008) suggestion that places, like people, have personalities that may result from the concentration of particular personality types. Alterations in the physical environment and human capital influence are also understood in relation to the socio-economic vitality of the resort. The chapter also relates how the current understanding of resorts' spatial structures and lifecycle evolutionary constructs are both exogenously and endogenously determined (Agarwal, 1997) and are responsive (Darwinian) and adaptable (Lamarckian), whereby the two evolutionary constructs operate in tandem, albeit at different levels and at different times.

The thesis concludes by offering findings which promotes agency being in charge of the environment where the structure is affected by the socioeconomic and cultural climate caused by external and internal forces. The agency influence at a local level is thereby critical in determining the meme (an element of a culture or system of behaviour passed from one individual to another by imitation or other non-genetic means) (Dawkins, 1986) that produces the place-specific outcomes. As Bexhill-on-Sea's readjustment to being a retirement seaside town may have worked well in compensating for the loss of tourism and education, secondary data and statutory agency representatives raise questions as to whether the town in its current form will be sustainable in the future. Middle-life retirees have brought with them their own personalities, which reflect the town as a quiet, safe, non-vibrant and limited economy labelled a 'Costa Geriatric' seaside town. This image and presentation of the physical environment and the human capital occupying the town are perceived differently by key agencies and middle-lifers, depending on one's personality and reflexive habitus, while where one positions oneself is a key finding in the thesis. The examination of perspectives on the physical environment, key agencies and middle-life migration as well as associated perceptions, images and labels has added knowledge to understanding resorts and their socio-economic and cultural evolution, which may assist in the future regeneration and modelling of principal English seaside resort towns. 


\section{Chapter 2}

\section{CURRENT UNDERSTANDINGS OF PRINCIPAL ENGLISH SEASIDE RESORT TOWNS}

\subsection{Introduction}

This chapter seeks to examine critically the literature analysing the problems that have affected England's principal seaside resort towns (Figure 2.1), determine what is known about them and construct the gaps in this knowledge. It establishes the boundaries of current understanding and examines the complex and multifarious arguments on these places. Furthermore, the chapter analyses the evolutionary and regeneration landscape within seaside resort towns as well as the issues facing these destinations and their transformation into other roles, in particular as dormitories or retirement centres.

Agarwal and Shaw $(2007,1)$ describe seaside resorts as 'old forms of tourism, catering for Fordist forms of consumption based around mass tourism'. Urry's (2002) discourse on the decline of the seaside resort embodies this notion, claiming that resorts have not shared in the recent growth of tourism and the decline has occurred in contrast to other destinations which have 'become the new leisure spaces, re-fixing the tourist gaze so that resorts are no longer extraordinary' (Urry, 2002, 36). Agarwal (2005) and Beatty et al., (2008), however, make the point that not all principal English seaside resort towns are in decline, while Walton $(2000,196)$ argues that 'instead of discussing resort decline, we should be trying to explain the British seaside survival', highlighting the spatial unevenness of resorts' economic performance, indices of multiple deprivations and changing structures. Agarwal (2005) questions why some principal seaside resort locations succeed and others fail, as they all are subject, more or less, to the same set of exogenous challenges as presented by authors such as Shaw and Williams (1997), Brodie and Winter (2007) and Benedict and Houghton (2009), and also emphasises the impact of endogenous actions. 


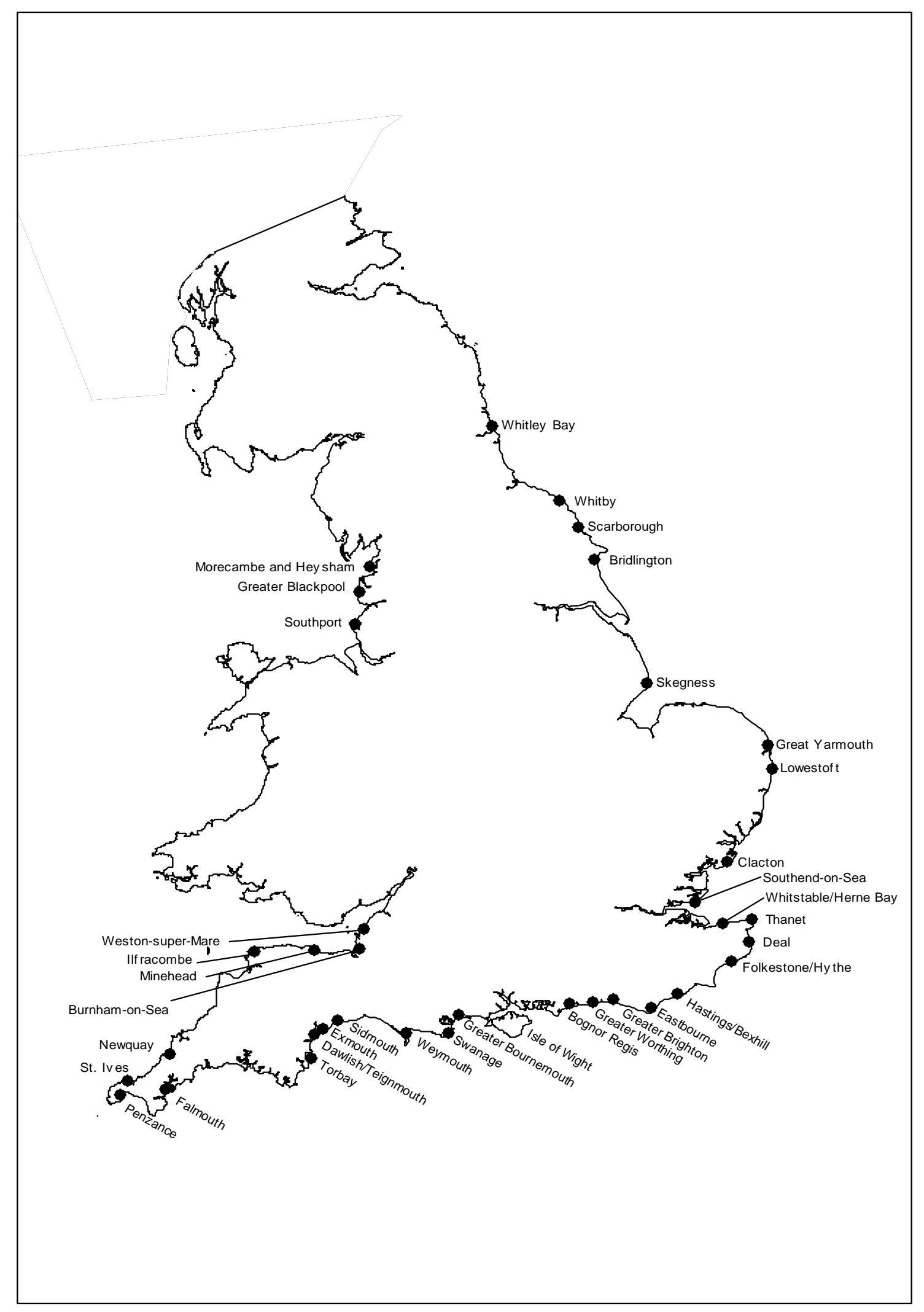

Figure: 2.1 England's 37 Principal seaside resort towns

'Source: Beatty et al., (2008)'

For those seaside resorts reaching maturity and consolidation, Butler's (1980) observed Tourism Area Life Cycle (TALC) destination model suggests that 
resort destinations will follow a path of stagnation and decline. Subsequent studies that have attempted to support this view have been undertaken from relatively narrow economic and historical perspectives (Walton, 2000; Urry, 2002; Beatty and Fothergill, 2004) and have neglected the socio-cultural aspects of seaside resorts and their development (Agarwal and Shaw, 2007). Other studies, by Agarwal (2002, 2006), Cooper (2006), Ravenscroft and Hadjihambi (2006), Russell (2006a) and Agarwal and Shaw (2007), have challenged the observed inevitability of the lifecycle model and contend that the resort destination lifecycle can be interrupted and managed to avoid decline, through intervening in the environment, key agency actions, leadership and entrepreneurial qualities or the migration of populations to a destination. Shaw and Agarwal (2007) maintain that academic and political interest in seaside resorts has only recently emerged and begun to explore and understand the socio-economic forces in detail, thus reinforcing this neglect of socio-cultural dimensions and the impact of migration to seaside resort towns.

\subsection{Declines in English seaside resorts}

The main body of literature contends that England's principal seaside resort towns have seen a gradual socio-economic decline (Walton, 2000; Brodie and Winter, 2007), due to a range of complex issues (Shaw and Coles, 2007) such as growth in car ownership, mobility factors, cheap overseas flights and generally more choice in spending the leisure/retail pound (Shaw and Williams, 1997). Traditional resorts, with their out-dated tourism offerings, can no longer rely on previous captive markets to sustain their economy and an everincreasing number of holidaymakers and day visitors with the time and independence to explore destinations without being fixed to particular places (Walton, 2000). In addition, 180 degree catchments, weather dependency, higher maintenance requirements of the public realm and a reduction in rail networks have further compounded these problems (Shaw and Williams, 1997; Walton, 2000; Watten, 2000; Brodie and Winter, 2007). Negative perceptions, pockets of severe deprivation and urban design conflicts have, amongst other factors, contributed to structural change in seaside towns (Beatty et al, 2008; Shaw and Coles, 2007; Agarwal, 1999; Walton and Browne, 2010), while the advent of cheaper housing in a poor state of repair within seaside towns has 
attracted a less well-off and economically inactive ageing population (Beatty et al., 2008; Margate Renewal Partnership, 2008; Government Select Committee, 2009).

In the 1990s, the decline of many seaside towns was very stark. Urry's (1987, 1997) view on the declining popularity of the English seaside resort was that the attributes of the seaside were no longer sufficiently special, and better quality experiences were available closer to home and also overseas. Considered to be at the end of their tourism area lifecycle (Butler, 1980), English resorts were transforming into dormitories and retirement centres (Walton, 2000).

However, even while visitor numbers shrank, the towns themselves grew in population numbers (Walton, 2000) as retirement migration took over from the holiday industry as the main driver of growth (Stallibrass, 1980). And whilst seaside towns have long been popular places as retirement destinations (Shaw and Williams, 1997), there is a recognition that concentrations of ageing populations has a significant impact for wider economic and social policymaking (European Policies Research Centre, 2006). For instance, the Lincolnshire Coastal Action Zone (CAZ) Partnership $(2006,23)$ argues that 'seaside towns suffer from 'rurban' problems - the worst aspects of rural and urban deprivation, created by such factors as in-migrating elderly people'. This can be compounded by transitory youth populations (seeking accessible housing opportunities) while at the same time losing those moving on to higher education and not returning. This can create frail and stagnant economic conditions mainly as a product of employment that is seasonal and low income service sector jobs, compounded by detachment from major settlements (Beatty et al., 2006; DCLG, 2007c; Walton and Browne, 2010).

The research literature, however, identifies not only these exogenous forces at work but also the endogenous actions of certain key agencies which influence structures and the physical environment. In turn, these affect people both visiting and living in seaside towns. Human social, cultural and economic capital shapes the personality of a place through migrants in search of work, living on benefits or moving for retirement purposes (Agarwal, 1997, 1999, 2002, 2007; Walton, 2000, 2009; Beatty and Fothergill, 2003, 2004, 2007; 
Beatty et al., 2003, 2006, 2008; Shaw and Williams, 2004; Thomas and Dorling, 2007). Only recently has there been a greater insight into seaside towns' economic performances, with a notable number of papers presenting some interesting statistical findings on change, not least 'The Seaside Economy' report (Beatty and Fothergill, 2003), 'England's Coastal Towns, A short review of the issues' (Fothergill, 2007) and 'The Coastal Communities of Southeast England' (Fothergill, 2012). Nevertheless, these findings tend to offer only a statistical analysis of change and not an interpretation of the socio-cultural and economic dynamics that occur in seaside resort towns and which require further investigation and understanding.

It is widely accepted that many of the principal English seaside resort towns are at the point of stagnation and decline and are unable to kick-start new growth. In this respect, Butler's (1980, 2005a, 2005b) TALC empirical model 'exploration, development, maturity, stagnation and decline' - observes the dynamics of resorts by working through a lifecycle. However, recent studies have moved away from the empirical TALC model towards exploring connections between the physical (human-made, built and urban) environment, migration and policy process involved in seaside resort change (Agarwal, 2002, 2006; Ravenscroft and Hadjihambi, 2006; Benedict and Houghton, 2009). Thus, adjustments to the physical urban environment appear to be an important factor in shaping seaside towns (Smith, 2002; Beatty and Fothergill, 2003; Smith and Denholm, 2006; Florida, 2008). A good example of this is the university status awarded to Bournemouth in 1992, attracting 17,500 students a year and changing the demographic profile of the town. Adaptations to the resort's physical environment can be facilitated by way of government intervention (national and local), through community leaders, by entrepreneurs or by corporate commercial companies (Taylor, 2000, 2003, 2007; Grimshaw and Smith, 2007; Tallon, 2010).

Agarwal's $(1997,72)$ study of Torbay concluded that 'the life-to-death concept outlined in the resort model is extremely problematic. No evidence was found to indicate the occurrence of irreversible decline that the model suggests'. In addition, post-stagnation phases seemed to be characterised by attempts at reorientation and rejuvenation to provide for altering markets. Therefore, despite 
the limited analysis of the three resort towns in Torbay (Torquay, Paignton and Brixham), Agarwal $(1997,72)$ concluded that 'regeneration and rejuvenation are an on-going process; resorts must continually re-orientate and rejuvenate their product, in order to keep abreast with competing destinations and constant market changes'.

The Margate Renewal Partnership study (2008), however, suggested that the complex interlocking problems of a weak labour market, an unbalanced housing market, high levels of deprivation and population transience have created very unstable communities and made it extremely hard for local service providers and regeneration practitioners to put Margate on a more sustainable and prosperous path (Benedict and Houghton, 2009). Thus, Urry's (1995) argument that some seaside resorts are at the end of their natural lifecycle may be justifiable in certain cases, in that many have reached such a point in the reduction of their facilities, both absolutely and in comparison to their competitors that they have little chance to compete and rejuvenate. He goes on to suggest that a factor contributing to the decline of the resorts is partly cultural. Changes in fashion, style and taste and people's values, beliefs and identities have had a significant effect, and seaside towns have become places to avoid rather than visit. Their competitive disadvantage is not just the result of the quality of investment, accommodation or the number of attractions - it is because there have been certain socio-cultural changes that have left seaside towns less valued within the English culture than they once were and that they are now largely visited by comparatively less wealthy people, the elderly and those groups who it seems cannot afford trips elsewhere. In contrast, the countryside has been rebranded as a 'good taste' destination (Urry, 1994, 1997). These changes, in turn, have occurred alongside, but are not reducible to, a shift in the regime of accumulation in advanced capitalist societies, from Fordism (characterised by mass production and consumption, standardisation and high-volume sales with attendant economies of scale) to post-Fordism (characterised by flexible/smaller-scale production, increased market segmentation and more individualised consumption) (Jayne, 2006). In short, the tourism area life cycle model (Butler, 1980) overlooks the structural changes or 'universal processes' that are ultimately responsible for the rise and fall of destination areas, primarily through economic restructuring and socio-cultural 
change (Agarwal, 1994, 1997, 1999a, 1999b, 2002; Berry, 2006; Agarwal and Shaw, 2007). It is this socio-cultural change in seaside towns that needs further examination and exploration.

Agarwal $(1999,2007)$ showed the importance of key statutory agencies in shaping, both positively and negatively, seaside resort towns through their actions. The first element in this respect is the failure of the public sector to recognise what has happened or is currently happening and then responding too slowly and putting in measures that have little or no effect. For instance, they have allowed planning applications for large numbers of flats, retirement homes or bungalow retirement estates. In addition, they have not promoted regeneration on the basis of political expediency, i.e. in order not to upset the local electorate who voted them into power (Shaw and Williams, 1997; Porter, 2004; Atterton, 2006; Walton and Browne, 2010). Secondly, other causes have been partly induced by private sector operators such as small- and mediumsized tourism enterprises (SMTEs) - 'phase-changing' entrepreneurs who, unable to make profits in the tourism sector, have re-orientated their businesses through local authorities allowing them to convert tourist accommodation, in order to attract in-migrants on incapacity benefits or pensions, thus damaging the very image of the destination (Agarwal, 1994, 1999; Demetriadi, 1995; Benedict and Houghton, 2009; Walton and Browne, 2010). Thirdly, larger corporations have taken their investments elsewhere inland or overseas (Urry, 1994; Russell and Faulkner, 2004). Moreover, the emotive English attachment to the seaside has made it difficult for these negative messages to penetrate the layers of affection (Walton, 2000), and so the response by national and regional government bodies has been slow. More recent actions by statutory agencies have seen the initiation of a number of socio-economic deprivation and regeneration programmes in selected seaside towns, but these seem to have had mixed results (SeaSpace, 2003a, 2003b; Margate Renewal Partnership, 2008; Smith, M.K., 2007; Tallon, 2010). Therefore, the constructive and/or damaging adaptation of the physical environment by key agencies appears pivotal in the subsequent life and evolution of resorts. 


\subsection{Postmodern interpretations of seaside resort towns}

As English seaside resort towns developed, so they came to 'specialise' in the provision of services to distinct social groupings. Some resorts were 'collective gaze' (mass) in nature, while others wanted a more 'romantic gaze' (individual) (Urry, 2002). Perkin (1976) argues in Urry $(2002,22)$ that 'the effect of the local elites on the respective 'social tones' of different resorts resulted from the way in which land and buildings were locally controlled and owned'. This produced

a complex 'resort hierarchy' which, by the twentieth century, 'satisfied a wide range of aesthetic preferences and catered for almost a complete cross section of society' (Walton, 2000, 27). However, the contrast between the 1950s and the twenty-first century seaside resort was that in the 1950s, tourism in Britain was organised around a resort hierarchy in which different seaside towns were visited by different social groups (Brodie et al., 2005, 2007). The situation now is that there is a 'consumption spaces hierarchy' that includes some resorts but also includes inland leisure sites (Alton Towers), towns and cities (Oxford), shopping centres (Blue Water), country areas (1066 Country) and heritage centres (Wigan Pier), as well as the ability to stretch across into Europe (Disneyland Paris) and the rest of the world. These are all new consumption spaces - spaces that compete to sell both themselves and numerous other forms of consumption (Zukin, 1998). Also, resorts such as Bournemouth have repositioned themselves, attracting a more youthful market by becoming a university town and changing their image. Some resorts in the 1990s made clear decisions to focus more on market segments, for instance Blackpool with its hen and stag night adult venues, Newquay targeting the niche surfing markets, Eastbourne with its retirement coach travel trade and St. Ives on art and culture.

Urry (1994) argues, resorts are weakly placed, since they flounder at the bottom of the consumption spaces hierarchy. Urry $(2002,85)$ goes on to suggest that 'resorts are no longer 'extraordinary', almost everywhere has become a centre of 'spectacle and display' and as a result resorts have relatively little to distinguish them from anywhere else' (other than perhaps being by the seaside). The division is now less clear-cut, as pleasure can be enjoyed in very many places with a proliferation of objects on which to gaze, and not all are 
concentrated at the seaside (Zukin, 1995; Urry, 2002). What seems to be presented by authors such as Agarwal and Shaw (2007) and Shaw and Coles (2007) is that seaside resort experiences are extremely diverse.

Whilst seaside historians have placed great emphasis on the history of the physical infrastructure and natural environment as determining resort lifecycles (Walton, 1983, 2000; Shaw and Williams, 1997; Watten, 2000), it is in the past four to five decades of decline that have shown that resorts are also shaped by the people who visit, migrate to (for business, lifestyle and housing reasons) and live in them (Demetriadi, 1995; Agarwal, 1999, 2002, 2007; Agarwal and Brunt, 2006; Margate Renewal Partnership, 2008). A review of the regeneration literature identifies that the migration of populations, whether the elderly, benefit claimants or commuters (Fielding, 1988, 1992; Findley et al., 1992; Beatty and Fothergill, 2003; Beatty et al., 2006, 2008; Margate Renewal Partnership, 2008) can have both positive and negative effects on seaside towns. For example, in the process of migration, housing in the area may undergo significant price adjustment, either upwards or downwards, dependent on the nature of migration. This transition also normally involves an element of tenure conversion whether from rental to ownership or vice versa and concurrently along with a physical change there is an economic, social and cultural phenomenon (Hamnett, 1991).

Florida's $(2008,13)$ observations on the importance of place-based dynamics are important here: the author states that 'today's economic factors - talent, innovation and creativity - are not distributed evenly. They concentrate in specific locations, in what is called 'clustering'. New ideas are generated and productivity increases when we locate close to one another'. This clustering force, therefore, makes some places more dynamic and industrious and so generates increased outputs and prosperity (Florida, 2007). The location in which people decide to live can have, as a result, enormous effects, in that it can determine the people one meets, the friends one makes, the choice of partners, the level of income earned and the options available to children and families. It is recognised that some places offer a better quality of life than others and that people are not equally happy everywhere. These places may offer, or give access to, better career opportunities, more vibrant labour markets 
and earnings opportunities, greater house price appreciation and promising relationship markets, or better environments for families (Nickell, and Quintini, 2003; ELSA, 2003, 2006, 2008; Florida, 2008; DCLG, 2006a, 2006b, 2006c, 2007b, 2008a, 2008b, 2009a, 2009b, 2009e).

People can also shape the personality of places, and the towns within which people live can be affected by social, economic and political conditions and how they interact and interrelate with the personality traits of the region (Agarwal, 1999, 2005, 2007; Agarwal and Brunt, 2006; Florida, 2008). Seaside town leaders and key agencies, therefore, need to be aware of this and what Florida $(2008,213)$ describes as 'how their collective personalities shape the kinds of economic activities and the kinds of people they can attract, satisfy and retain' and that 'people seek out places to live that reinforce and reflect aspects of who they are and who they want to become' (Florida, 2008, 204). Therefore, understanding the reasons for migration is important, but never more so than in the twenty-first century, as geographical location will determine personalities and at the same time separate out the 'haves' and 'have-nots' (Bailey et al., 2006; Bailey and Livingstone, 2007). This changing physical environment and the 'New Economy' (Perrons et al., 2005; Florida, 2010) may therefore focus leaders' thinking on structures and agencies and on building opportunities for a middle-life workforce.

Florida (2004, 2005, 2007, 2008; Florida and Mellander, 2007) also attaches importance to the 'personality of place' concept and its relationship with and connection to human life courses. When young, a stimulating environment with a great nightlife and vibrant 'mating market' is important, along with good job opportunities. With marriage and children these priorities change, and so a safe environment, good schools and better family facilities take precedence. When children leave the house the parents' 'empty nester' needs and interests change yet again (Florida, 2007, 2008; Thomas and Dorling, 2007; Dennett and Stillwell, 2009). Place seems to matter even more today than it did before. Despite all those predictions about new technologies - the car, the cell phone and internet - freeing us from our locations, allowing us to work all over the world and enabling us to live pretty much anywhere, location remains a key factor in the global economy. The clustering force has resulted in the 
geographic concentration of personality types, where different kinds of places suit distinct types of people (Florida, 2008). A study by Thomas and Dorling (2007) shows the powerful role location plays in our class position, health status, education options and economic mobility. The study found that people born in disadvantaged locations tended to carry that initial disadvantage across subsequent life stages. They concluded that this new geography of class regarding where you live can limit or assist your life chances, from cradle to grave. Today, location constitutes an additional divisive line that separates the 'haves' from the 'have-nots', alongside race, education, occupation and income (Bailey et al., 2006; Bailey and Livingstone, 2007).

What appears to matter today, therefore, is not where most people settle, as was the case in agricultural and industrial economies (Champion, 2005), but where the largest numbers of the most skilled people locate, because highincome earners bid up the price of housing and land (Florida, 2007). It is generally not the local labour market generating too much demand but a combination of desirability and increased mobility and in-migration that drive house prices upwards. In areas where housing values are already high, these high earners make regions that were already ripe for growth even more desirable - and to a greater number of people (Gyourko and Sinai, 2001, 2006). Along with this desirability, so the aesthetics of the location matter a great deal, and so the higher the aesthetic value, the higher the level of overall community satisfaction, as evidenced in the UK government's 'Place Surveys' undertaken by Ipsos/MORI for all local authorities (Appendix 1). As Coles and Hall (2006, 289) state, 'matters of location and place cannot be isolated from the relations and linkages between spaces and hence the associated mobilities of capital, people, ideas and images'.

Understanding the motivations behind middle-life migration is therefore extremely important, as these people perhaps seek to downsize and use their wealth to locate to a desired location (Rowles and Watkins, 2001; Godfrey et al., 2004; Gurran, 2008). 'Means' migration, where migration involves the pursuit of work (Champion, 2005), severs the long-held connection between population and economic growth, and so what appears to matter is to where the greatest numbers of the most skilled people locate (Florida, 2008). Middle-lifers 
have shown a desire, or need, not to retire, and therefore they may seek to locate where there is access to employment opportunities and clusters of likeminded people, personality types and places that offer amenities and quality of life (Rowles and Watkins, 2001; Florida, 2004, 2005, 2007, 2008; Longino and Warnes, 2005). However, some of these moves have not been completely positive, and places have become labelled through the types of people and personalities imposed on a location, for instance Margate's 'Costa del Dole' (Margate Renewal Partnership, 2008), Bexhill-on-Sea's 'Costa Geriatrica' or Eastbourne's 'God's waiting room' (Shaw and Williams, 1997; McHugh, 2003; Argus, 2005, 2009). As Shaw and Coles $(2007,47)$ comment, 'such mobility flows are reshaping the social ecology of resorts and creating much more divided communities as well as a series of difficulties. These relate to an increase in social care problems, a transient, low-skilled population and housing difficulties, along with a growing elderly population'.

With life expectancy in England at 78 for men and 82 for women (Appendix 2), and with a third of the population in England now aged 50 or over and forecast to grow exponentially to 41 per cent by 2031 (ONS, 2008, 2009), this has implications for services and the political environment in which the seasidebased local authorities operate. Appendix 3 shows the mid-term estimates and age distribution for England's principal seaside towns - other than Brighton, the share of population over state pension age in most cases is considerably higher in the southeast of England (34 per cent for Bexhill-on-Sea) compared to other English seaside resort towns (24 per cent), England as a whole (19 per cent) and the southeast generally (19 per cent) (Beatty et al., 2008).

\subsubsection{Middle-life migration as a process shaping seaside towns}

Understanding retirement migration motivations appears to be a central theme in improving seaside resort towns. Nostalgia, notions of the rural idyll and images of travel and tourism (Urry, 2002; Van Dam et al., 2002; O'Reilly, 2007a) are the underpinning motivations for migration. Therefore, understanding how to encapsulate and present these elements in the physical and natural environment, and stimulating the local population to take on board the regeneration and resurgence of seaside resort towns, may be the key to 
unlocking the stagnation and decline in these locations. However, retirement or semi-retirement will take on greater significance and meaning, while evident connections between those people with better education, better health and more wealth, who are seeking continuation in employment, and those who are less educated, healthy or wealthy taking early or forced retirement and not reentering employment (Hirsch, 2003; ELSA, 2008; Gurran, 2008) will be an important factor in determining where middle-lifers choose to locate, as parttime, flexible employment opportunities may become factors in their migration decision-making. Therefore, images of seaside resort towns beyond tourism, retirement and benefit claimants may need to embrace and intertwine lifestyle and means migration, if they are to experience rejuvenation or reinvention.

Florida (2008) and Thomas and Dorling (2007) suggest that specific age cohorts reflect different traits, such as the current elderly (over 70 years of age) with their 'agreeable and conscientious' personalities and cautious, thrifty spending who, according to seaside historians have downsized and retired to the seaside based on nostalgia and a status quo mentality (Walton, 1983, 2000; Shaw and Williams, 1997; Atterton, 2006). Sweetman (2003) also presents that within the age cohorts their class disposition, education and economic capital contributes to how they perceive a place. However, the baby-boomer generation that is now moving into middle-life (50-70 years old) and 'retirement' has very different attitudes and life experiences to past retirees (Evandrou, 1996; Barnes et al., 2004; Evandrou and Falkingham, 2006). According to Bauman (1998) they are not defined by broad social economic demographic structures but by consumer lifestyles and a 'work ethic' (Scott, J., 2000; Clarke, 2003; Lancaster and Stillman, 2003). Furthermore, they now live in socioeconomic conditions that will require continuation in employment beyond the traditional state pension years (ELSA, 2003, 2006, 2008, 2010; Blow et al., 2004; DWP, 2005, 2009a; Longino Jr. and Warnes, 2005; Miles and Miles, 2004; Evandrou and Falkingham, 2006; Florida, 2008; Twenge et al., 2010).

Many seaside resort towns' population growth is driven by in-migration (internal migration) rather than by natural increases through births. To understand these net migration movements in more detail, Dennett and Stillwell (2009) used Vickers et al.'s (2003) National Classification of Local Authority areas (Appendix 
4) to provide a summary (Appendix 5) revealing that different age groups differ markedly (Appendix 6). Appendix 7 places the top 10 in-migration and outmigration rates between pairs of classification groups in which coastal Britain (B2) ranks at the top of in-migration rates in the '45-state pension age' and 'state pension age and above' and, that in-migration is predominantly from rural Britain (B1) to coastal Britain (B2). It may well be that migration patterns to coastal Britain or from/to rural Britain are, in part, linked to short, cross-political boundary moves. This will be considered later in the thesis when migration patterns in Bexhill-on-Sea, East Sussex are reviewed. Fotheringham et al., (2004) note that a contributory factor to the relationship between out-migration rates concerns local economic conditions, in that out-migration rates are relatively low from economically depressed regions and relatively high from economically prosperous areas. In prosperous regions with low unemployment rates, job increases have to be met through in-migration of labour from other regions. Conversely, in depressed regions, with high unemployment rates, job increases can be met internally. Hence, there is this seemingly counterintuitive trend whereby areas with high scores on indicators of deprivation have low volumes of out-migration. Relatively deprived areas have lower out-migration rates than more prosperous areas, because of a low turnover effect.

Research by Dennett and Stillwell (2009), Vickers et al., (2003), Champion (2001), DTZ/Pieda (2006) and DTZ (2010) evidenced that middle-lifers tend to migrate to rural areas, but as they get older they migrate to seaside towns, with most moves undertaken after careful and extended thought and planning, and frequently lower housing and living costs as important factors. However, with a bulge in the demographic of middle-lifers and housing demands in rural areas, especially in south and southeast England (RDC, 2007a; GOSE, 2009), this may create pressures, or opportunities, for middle-lifers to consider migrating to seaside locations sooner than they may have originally envisaged. The literature review identified that middle-lifers' migration motivations may well change as 'means' and 'amenity' migration to support their accustomed lifestyles, could play a greater part in their decision-making. Amenity migration is a complex mix tied up in motivations, but it is generally perceived as happening when 'migrants move for lifestyle, rather than jobs, choosing places with natural amenity, climate, recreation, and affordable housing' (Gurran, 2008, 
391). Therefore, amenity migration can often be perceived as a place where people have a greater environmental quality, differential culture and higher quality of life (Rowles and Watkins, 2001; Gurran, 2008). Whereas, if 'means' migration, where 'migration is for work' (Champion, 2005), is to become an important motivation for middle-lifers in the future, then the traditional retirement migration flows for this age group may look distinctly different and migration decisions may take on greater and wider significance. This geographical mobility is however becoming increasingly complex, and 'lifestyle' migration, described by Benson and O'Reilly $(2009,1)$ as 'where the search for personal fulfilment by the affluent, geographically mobile, through an individualist concept is fashioned by the global rather than the personal,' may become increasingly important in influencing how destinations present themselves and the perceptions generated externally.

The work by Florida $(2008,2010)$ implies that 'means' migration motivations and decisions are partly influenced by being well-connected to opportunities for employment as well as by the clustering of creative skills and innovative people. Thus, the longer-run issues facing these places have as much to do with their 'psycho-social environment' as with their business climate and economic structure (Florida, 2008). People seek out places to live that reinforce and reflect aspects of who they are and who they want to become (Florida, 2007, 2008). Therefore, middle-lifers' migration patterns may shape a destination, but, importantly, they may also need forces that will attract them; their location choices may be shaped by their perceptions of a place, its housing, employment and consumption opportunities, amenities and aesthetics and a suitable psycho-social environment to meet their demands and expectations as they move into a new period of their life. Most people can no longer expect a single location to fit their evolving needs as they move from one life phase to another (Urry, 2002; Florida, 2007, 2008). Just as places have come to specialise in the kind of job opportunities they offer and the amenities they provide (Gordon and Goodall, 2000), most have also come to specialise in the stage of life they best fit (Florida, 2008; Dennett and Stillwell, 2009). Such mobility flows have shaped and continue to reshape the social ecology of seaside resort towns, and in some locations these mobilities have created much more divided communities and ongoing difficulties (Swarbrooke, 2000; Palmer, 
2002; Shaw and Williams, 2002; Garcia, 2004; Miles, 2005; Agarwal \& Brunt, 2006; Smith, M. K., 2007).

Florida (2008) contends that while opposites sometimes attract, most people are happier and more fulfilled in places that in some sense match their personalities (Thomas and Dorling, 2007; Florida, 2008). For instance, industrial areas tend to attract 'agreeable and conscientious' people, capable of working in mass production routine roles, whereas 'extroverted' types tend to locate where there are sales and service businesses, and 'open-to-experience' types are clustered in larger cities which are home to diverse economic structures. This overlay between personality type and economic structure has been distorted by globalisation (Florida, 2004, 2005, 2007, 2008; Thomas and Dorling, 2007) and the status quo orientation of many seaside resort towns that may have encouraged the out-migration of 'open-types' who tend to be a source of creative energy and innovation (Walton, 2000; Russell and Faulkner, 2004; Florida, 2008; Walton and Browne, 2010).

Town leaders and key agencies must acknowledge how their collective actions shape the kinds of economic activities and people they can attract, satisfy and retain (Russell and Faulkner, 2004; Florida, 2008). For many seaside towns, it appears their early history had a prevalence toward 'open-to-experience' personality types (Thomas and Dorling, 2007), bringing entrepreneurship into these locations. However, it seems that these personality types have since moved or passed on, and they have been supplanted instead by the elderly and retired. This cohort has shown more industrial traits of 'agreeable and conscientious' personalities (Thomas and Dorling, 2007) who came through the 1930s Depression and war years and carry with them life experiences of cautious and thrifty spending (Boaz et al., 1999; Blow et al., 2004; Florida, 2008; Twenge et al., 2010), retiring from their industrial and manufacturing backgrounds to the seaside based on nostalgia with an underlying pervasiveness for stability and the status quo (Shaw and Williams, 1997; Florida, 2008; Benedict and Houghton, 2009). Some seaside resorts, such as Brighton, appear to have recaptured the 'open-to-experience' types bringing innovation and creativity to the area (English Tourism Council, 2001; CABE, 2003; English Heritage, 2007), whereas others remain labelled with very 
different personalities, attracting 'agreeable and conscientious' types who are capable of working in mass production or in routine roles, such as in the care industry and public sector. Consequently, in recent decades, interventions by political and key agencies in urban regeneration and the physical environment have more frequently been met by this elderly retired population with opposition to change (Walton and Browne, 2010), which has led to a gradual decline in some resorts. These cultural personality traits can take years, decades and in some cases generations to alter, and therefore they need careful consideration by key agencies and require further examination in relation to seaside towns.

Resorts are therefore dynamic places that change over time, and when viewed at the macro level they exhibit the characteristic life/death pattern of the TALC model (Butler, 2006a). This trait is evident in Bexhill-on-Sea, but when focusing on the evolution of a destination it can be observed that there are many variations (Haywood, 2006a), and Bexhill-on-Sea is one such example. Agarwal (1997, 1999, 2002, 2006), Coles et al., (2004) and Cooper (1995, 2006) have shown that the extent to which resorts are viewed depends on the complex dominating forces, such as organisational, political and economic, that are at work. The impacts that these forces have on a resort destination may not be readily apparent, and that insufficient time has been spent learning how the interplay of structure and agency affect these forces that causes a destination to decline (Haywood, 2006a; Milne, 1998; Williams and Shaw, 1998).

Thus, the actions of key agencies are notably important in determining a destinations success or failure insofar as they alter the environment or attract socio-economic and cultural groups that impact on the economy of the resort. Zimon (2000) suggests that rather than a deterministic 'lifecycle', the TALC is viewed as part of a larger evolutionary epistemology where tourist systems alter according to active changes in the environment.

\subsection{Evolution and resort lifecycles: Lamarckian analysis}

English seaside resort development took place predominantly in the nineteenth century through entrepreneurial landed gentry who invested in creative and innovative projects around the coast - some successful, others not. Local 
authorities followed in the twentieth century by investing in the public realm, which was driven by elected members 'acting-out' their opportunity to be 'municipal capitalists', or entrepreneurs 'infiltrating' the council and influencing the spending of taxpayers' money to help supplement the destination industry provided by the private sector (Walvin, 1978; Walton, 1983; Shaw and Williams, 1997; Russell and Faulkner, 2004; Brodie and Winter, 2007; Walton and Browne, 2010). In a number of case studies it appears that the 'revitalising' entrepreneurs that followed in the latter half of the twentieth century seemed to set the next direction, both positive and negative, of the seaside resort towards either new growth or decline (Agarwal, 1999, 2002, 2007; Russell and Faulkner, 2004; Agarwal and Brunt, 2006; Beatty et al., 2006; Agarwal and Shaw, 2007; Margate Renewal Partnership, 2008). However, in today's world, 'corporate' entrepreneurship now dominates, and as a result some of the creativity, innovation and vibrancy of the past may have been lost (Elliott, 1997; Russell and Faulkner, 2004; CABE, 2010; Hearn, 2010) and so individual entrepreneurs find it more challenging to instigate a change in the direction of development than perhaps in the past (Russell and Faulkner, 2004). Studies have shown that for entrepreneurs to succeed, a supportive culture in the destination must be in place, in order to create the right conditions (Mueller and Thomas, 2000; Russell, 2006b) whereby a local champion is seen as the single most important success factor (Russell, 2006b).

Hernández et al., (2007) assumed that resort destinations can be determined by both physical and natural capital. Physical capital includes aspects such as the transport, infrastructure and services provided by the resort, while natural capital can be found in the environment. Therefore, they approach the destination as being shaped by the physical and natural environment rather than necessarily human content. Their lifecycle model, adapted from Butler (1980), follows a predator-prey concept, whereby physical capital (predator) requires natural capital (prey) to be sufficiently attractive to exert a pull on visitors, though the natural capital of the area can be spoiled by growth in infrastructure and excess visitors. The authors go on to say that 'if the natural capital maintains its initial value then the evolution of the physical capital would be explosive. An initial level of infrastructures would attract tourists to the location, bringing in revenues that would be reinvested in physical capital 
attracting more tourists and so on. Physical capital would only decrease through its depreciation rate' (Hernández et al., 2007, 9). However, they also state that 'the natural capital can/will be affected by the development of the physical infrastructure potentially damping the explosive behaviour of the physical capital' (Hernández et al., 2007, 10). Thereby, they suggest that the 'tourism impact' is determined by both physical and natural capital.

Some authors present a picture of intervention in the physical environment. Lamarckian studies suggest that changes in the environment cause changes in the needs of organisms living in that environment, in turn causing change in behaviour (Ravenscroft and Hadjihambi, 2006). Some seaside towns have shown traits of rejuvenation and new phases of growth (Urry, 1995; Agarwal and Shaw, 2007), such as in the south of England in Bournemouth and Brighton, while others have shown signs of decline, such as Blackpool and Torbay (Beatty et al., 2008), through alterations to their physical environments which attract different human social, cultural and economic capital. Therefore, the Lamarckian interventionist perspective, according to Ravenscroft and Hadjihambi $(2006,159)$, is 'more a result of a complex series of external environment planning and development decisions' by key agencies, rather than natural evolution. This contradicts the temporal inevitability of the lifecycle model, in favour of an open-ended approach that links resort survival to adaptation (Ravenscroft and Hadjihambi, 2006). This approach is predicated on the ability of key agencies to develop a series of improvements to the physical environment that allow it to adapt to and alter the nature of local demographics. This seems to be a critical point of examination.

Butler's (1980) TALC has been discussed as a conceptualised evolutionary cycle. In examining this further, in relation to urban environment regeneration and people, Ravenscroft and Hadjihambi (2006) make the argument that Butler's life cycle is Darwinian in character, whereas Lamarck's evolutionary perspective about learning and adapting to the environment may be better applied to understanding and managing socio-economic and cultural regeneration. Lamarck's work embraces environmental influences at a local level as one of the principal catalysts for change and adaptation. Lamarck offers a model for the survival of the best adapted, and as such he proposed 
that survival is a direct result of physiological intervention. So, for seaside town resorts, rather than the inevitable time-mediated life/death lifecycle process, as observed by Butler (1980, 2004), Ravenscroft and Hadjihambi (2006) suggest that development might be the result of a complex series of planning and development decisions analogous to environmental and physiological interventions, whereby specific interventions have the potential to subvert the Darwinian natural evolutionary model. It therefore appears that resorts might manage their lifecycle so that when they reach the maturity phase they are best placed to avoid stagnation and decline by adapting and intervening in the physical environment, to influence the lifecycle model. This management of the lifecycle has been identified as the product of a number of different agencies intervening at different stages of the lifecycle, for example the landed gentry, municipalities, innovative entrepreneurs or large corporate businesses. Understanding these key agency actions and their consequences requires a good deal of further examination.

Theoretical arguments, supported by some limited empirical data, suggest that the environment and human cultural activities have influenced human genetic evolution by adapting the foundations of natural selection and changing genotype frequencies in some human populations (Feldman and Laland, 1996; Martin and Sunley, 2007). The suggestion that acquired characteristics of an individual or institution can be passed on to or intimated by others has both its academic supporters and detractors and channels energies towards phenotypegenotype distinctions (Hodgson and Knudsen, 2006). The relationship between cultural and genetic evolution raises two connecting issues. The first is to what extent are present-day human cultures directed or inhibited by our biological evolutionary heritage, while the second concerns whether and to what extent human genetic evolution has itself been influenced by the environment and cultural activities. Authors, such as Laland et al., (2000), assert that these notions are inextricably linked. As Stankiewicz (2000) has observed, it is far from clear just how this process happens in technological development, or at what level. In contrast to biological inheritance, where it is the genome that varies internally, the resort development process is largely externally initiated and mediated. While Dawkins $(1986,1989)$ has proposed the 'meme' (an element of a culture or system of behaviour passed from one individual to 
another by imitation or other non-genetic means) as a 'technological genome,' it does not have the same characteristics or functions as the biological genome. Objectors to Lamarckian theorisation argue that while socio-economic and cultural transmission occur in human societies, this is not an 'inheritance of acquired characteristics' but merely a 'passing-on' and does not transcend to a genotype but purely a phenotype (Feldman and Laland, 1996; Laland et al., 2000).

Lamarck's work suggests that as the 'circonstances' (Butler, 1914) of their habitat, and exposure to their surroundings and mode of living changes the character, form, proportion, consistency and industry change proportionately (Packard, 2007). Packard (2007), in citing Dr J. A. Allen's (1871) works on North American species, showed that variations may occur through the actions of specific environments and that they become likeminded in manner to the conditions of the environment. There are connotations in this respect within the Margate Renewal Study, in that the changing environment of tourism-related infrastructure has perhaps altered the resort's character. So, in this context, if a resort's physical environment alters, then human social, cultural and economic capital become conditioned to that environment. As such, a common misconception about Lamarck's original works is that it was thought he believed in the environment being a direct cause of hereditary changes in organisms. However, in Philosophie Zoologique, Lamarck proposes the principal use or disuse of a certain part of an organism's body causing it to enlarge or shrink, i.e. 'the first law' (Butler, 1914; Packard, 2007). Lamarck maintains these changes are caused by the force of habits, so that conditions induce species to modify themselves accordingly (Butler, 1914). In addition, Lamarck maintains that change is caused by force of habit, so that, according to him, species are not modified by the conditions of their existence, but only induced by these conditions to set about modifying themselves (Butler, 1914; Packard, 2007). Thus, according to Lamarckian thought, socio-economic and cultural evolution are critical as agencies of change facilitated by changes in the physical environment, where structures affected by the environment alter and adjust the resident population (Packard, 2007; Ravenscroft and Hadjihambi, 2006). If regeneration practitioners and community leaders were to apply a Lamarckian evolutionary perspective to socio-economic and cultural theory on destinations, 
it should be possible for key agencies to devise strategies to prevent resorts from falling into decline and to kick-start new growth by intervening in the physical environment and subsequently attracting new people to the area.

With work appearing to become a fundamental part of middle-lifers' semiretirement plans (DWP, 2005), some seaside resort economies are developing year-round, mixed-use economies (Coupland, 1997; SeaSpace, 2003a) that may support this demand. The Hastings and Bexhill-on-Sea Task Force has adopted a 'top-down' strategy (SeaSpace, 2003a, 2003b) that promotes alterations to the physical environment through commercial land and property, in order to regenerate the area, and, so it appears, a Lamarckian view, by choosing the physical environment as one of the principal catalysts for change. Inherited Lamarckian traits involved in shaping seaside towns by changing the physical environment were evident in the case of the entrepreneurs the Bryant Brothers, who worked with Restormel Borough Council to redevelop parts of Newquay into a 'surfers' capital' (CABE, 2003; Brodie and Winter, 2007; English Heritage and Urban Practitioners, 2007). For Margate, the impact was different, in that the physical environment offered cheap accommodation for rehousing benefit claimants by London authorities; this led to a change in local demographics and a decline in the economic and social performance of the town (Margate Renewal Partnership, 2008; Beatty et al., 2009; Inside Housing, 2010). This gives substance to a Lamarckian socio-economic and cultural interpretation where agency is in charge of the environment and where structure is affected by the socio-economic and cultural climate (external and internal forces). Thus, Ravenscroft and Hadjihambi's (2006) Lamarckian socioeconomic and cultural perspective on their research of Ayia Napa, Cyprus, argues for a requirement to further examine these connections between the physical environment, migration and the policies of key agencies.

It is the resemblance to and processes of Lamarckian socio-economic and cultural evolutionary interpretation that challenge Butler's (1980) TALC observed inevitability and offer opportunities for regeneration practitioners whereby, affecting the physical environment they may be able to improve the socio-economic performance of the seaside resort town. 


\subsection{Regeneration and the 'New Economy'}

It took until 2006 for a government select committee to recognise a number of inherent socio-economic problems in seaside towns (Beatty and Fothergill, 2003; Beatty et al., 2003; DCLG 2007c, 2007d). With the current deep-rooted recession (Florida, 2010) it is likely the problems will become even more acute for these locations, just at a time when there are significant cuts in public expenditure and regeneration programmes (DCLG, 2010f). However, a few public sector regeneration programmes have targeted some seaside towns over the past couple of decades, for instance Margate, Blackpool, Torquay, Hastings and Bexhill-on-Sea (Watten, 1998; SeaSpace, 2003a; Agarwal and Brunt, 2006; Agarwal, 2007; Margate Renewal Partnership, 2008). However, in order for these towns to attract government regeneration grants, they had to present the worst of their area using indices of multiple deprivation (IMD) (DCLG, 2007b; Beatty et al., 2008; Government Select Committee, 2009), which conflicted with their need to promote positive images to attract visitors, new residential migrants and businesses (Government Select Committee, 2009; Walton and Browne, 2010).

For some seaside resort town regeneration practitioners, as stated at a seaside conference at Hastings, there is a buoyant view: 'The future looks bright, the future looks coastal' (Gaventa, 2009). This optimism may have come about because of the work by the government select committee (2009) on 'Future Policy Thinking', which made three recommendations for seaside towns' regeneration. Firstly, the Treasury and the Department for Communities and Local Government (DCLG) should support areas which contain disproportionately large numbers of elderly, vulnerable and transient households through reforms to existing government formulae and grant mechanisms. However, this has not materialised, and government grant settlements for 2010/11 and 2011/12 for seaside districts and boroughs was extremely poor, with cuts in grant funding, for many, of around 28 per cent (HMT, 2010; DCLG 2010f; Halcrow Group, 2010; RDC, 2011a). Secondly, it was recommended to implement seaside housing market renewal interventions on a scale that would alter underlying housing market dynamics. This is now unlikely to happen, as a number of quangos have been disbanded or 
downsized, thus making far fewer resources available (CIPFA, 2009; CIPFA/Solace, 2009; Boyle and Harris, 2009; DCLG, 2010c). Thirdly, investment in intensive government and voluntary outreach and support programmes was targeted at the most vulnerable 'high cost, high harm' families (Future Policy Thinking, 2009).

Whether urban regeneration happens through the gentrification of housing (Hamnett, 1991), migration and populations (Champion, 2005; Dennett and Stillwell, 2009), 'culture-led' flagship projects (Evans and Shaw, 2004), 'cultural regeneration' through mega events (Chaney, 1994; Tiesdell et al., 1996) or neighbourhood renewal (Taylor, 2007), there appears to be tension in socioeconomic and cultural studies, as some of the literature identifies that these approaches contribute to cultural inclusion, the celebration of diversity, the empowerment of community and increases in the quality of life (Booth and Boyle, 1993; Smith, M. K, 2003, 2007), while others believe that these approaches foster social polarisation, gentrification, social inequality and exclusion and the uneven distribution of costs and benefits (Swarbrooke, 2000; Palmer, 2002; Garcia, 2004; Miles, 2005; Smith, M. K, 2007). However, Florida (2010) simply feels this is a price that dynamic economies have to pay, and so this leaves key questions relating to social exclusion and polarisation that are relevant to urban regeneration and pertain to whether certain urban areas are becoming more unequal places, despite urban policy and regeneration (Tallon, 2010).

A chief objective of regeneration programmes appears to be the recreation of a sense of place (Smith, A, 2007), that promotes distinctiveness, quality of life, employment opportunities and community spirit. Government literature (DCMS, 2004; DCLG, 2006a, 2006b, 2008b, 2009b) claims that the community should be at the forefront of socio-economic development and more central to regeneration (Grimshaw and Smith, 2007; Smith, M. K, 2007). As Martin (2006) describes, the consistency with which the resident community supports or rejects regeneration partly depends on the nature of the people living in the town itself. His analysis of research at Hilton Head, South Carolina, evidenced that the nature of the retirees, most from well-educated and successful business backgrounds, led to an understanding of the importance of 'not killing the goose 
that laid the golden egg' (Martin, 2006, 246), and thus the community supported 'managed tourism', which went against the previous mayor's policy on abandoning tourism. Amongst all this complexity there also appears to be considerable mistrust of local government amongst communities, and while this can be overcome, it does take time (Purdue et al., 2000). Tallon $(2010,148)$ described how Taylor (2007) identified that many 'communities feel marginalised and unable to influence the decisions that affect their areas'. Taylor $(2003,2007)$ and Hovinen (2006) cite issues such as obstructions in the form of local power holders and government cultures, whereby communities are usually expected to adapt to the 'rules of the game' and often find themselves in an arena with which they are not familiar and do not understand. Decisions seem to be taken 'somewhere else', often within pre-existing authorities and structures. However, despite this criticism there is little evidence to suggest that community interest and participation do actually make a difference (Grimshaw and Smith, 2007). Capabilities, competencies and understanding of the bigger issues are also misunderstood and can vary amongst communities.

However, with the new Coalition government significantly reducing regeneration grants (DCLG, 2010a, 2010c, 2010g), their approach to regeneration is by way of private sector-led physical investment through Local Enterprise Partnerships (LEP's) (DCLG, 2010a) and through the agency debate of the 'Big Society' (Cameron, 2009), whereby government is encouraging individuals to be more active agents in their own lives. This interaction between the physical environment and human social, cultural and economic capital could possibly set the tone for the regeneration of resorts. The literature review identifies that regeneration programmes that promote these community aspects frequently rely on key public agencies, such as local authorities, to intervene if they are to affect the direction of the town and its tourism lifecycle (Lees, 2003b, 2008; Butler, 2004, 2005a, 2005b; Baum, 2006; Berry, 2006; Church and Coles 2007; Smith M. K., 2007).

The literature review identifies a number of seaside resort towns that are in severe decline and are seeking ways to halt and reverse this trend (Lever, 2007). Florida's $(2008,2010)$ observation is that locations are more likely to succeed if they are part of (or have easy access to) a successful economic 
'mega region' (Florida, 2008), and the southeast of England is recognised as one such area. The south coast of England is accessible to work, leisure amenities and consumption activities, and it is where seaside resort tourism began, founded upon health, well-being and serendipitous entrepreneurialism (Shaw and Williams, 1997; Russell and Faulkner, 2004; Agarwal and Shaw, 2007; Brodie and Winter, 2007; Beatty et al., 2008; Argus 2009). Agarwal and Brunt (2006) argued that seaside resorts located in the southwest of England are distinctly different to those in the southeast, as in the southeast they have more diverse economies, tourism is no longer the main foundation of employment and the resorts perform different economic functions such as acting as commuter settlements. It could be debated that the Hastings and Bexhill Regeneration Taskforce recognised this feature and identified approaches to revitalise the towns away from or complementing their tourism histories through a shared vision of co-existence: 'The securing of economic prosperity through the creation of a new urban fabric accommodating a vibrant resident, business and education community where the economic benefits can be directed to the most deprived wards in the communities of Hastings and Bexhill' (SeaSpace, 2003a, 6). Cooper (1997) and Baum $(1998,2006)$ argue that in some failing destinations it may be more appropriate to abandon tourism and how this may be influenced by communities forcing the political governance of the town to support that tourism no longer offers the best option socially, economically, culturally or environmentally or, perhaps even in 'lifestyle terms'. The options for a failing destination like Bexhill-on-Sea could be to exit tourism consciously, a move which Baum (1998) terms 'abandonment', though he stresses that emotionally this is a difficult decision for destinations. The second option, taken by many destinations, is a conscious decision to rejuvenate and adapt - and thus extend - their tourism lifecycle.

While it has been shown that quality of life and local cultural and amenity offerings are important factors in urban regeneration (Montgomery, 1995; Smith, M.K., 2003, 2007; Evans and Shaw, 2004; Miles et al., 2004; Atterton, 2006; DCLG 2009e; Ipsos MORI, 2009), a growing factor for middle-lifers' migration decisions is employment opportunities (Peterson, 2000; Heet, 2003; DWP, 2005, 2009b; Cetron and Davies, 2010; Whiteleaf, 2010). The view that the traditional extrinsic rewards of pay, prestige and material possessions motivate 
humans to work is being challenged (Brett and Stroh, 2003). A job that is stimulating, challenging and has an important impact on others is characterised as intrinsically motivating and is more in alignment with modern organisational theory. Working for work's sake in a job that is motivating and provides responsibility and variety appears to be a growing driver, rather than the traditional driver, to obtaining material or extrinsic rewards (Brett and Stroh, 2003; Lancaster and Stillman, 2003; Twenge et al., 2010). Work has even broader implications for society and communities, in that the workforce involved in service jobs, which are extensive in seaside towns, are less amenable to global competition and outsourcing, as it is hard to outsource the person who cuts your hair, mows your lawn or takes care of children or ageing relatives. These kinds of jobs are rooted firmly in locations, contribute significantly to the economy and give a positive self-image and identity and that is challenging, exciting and creative (Phillips, 2004; Moen, 2005; Powe and Hart, 2009). With the emergent 'New Economy', the future may react differently to the past as the nature of work changes (Peterson, 2000; Heet, 2003; Cetron and Davies, 2010; Whiteleaf, 2010). Workers are making less daily commutes, working flexible hours, working from home and telecommuting (Barnes et al., 2004; Phillips, 2004). With work set to become the norm for middle-lifers (DWP, 2003), migration and location decisions may take on greater significance for this cohort as they seek the converging of 'means' migration for work (Champion, 2005) with 'amenity' migration for lifestyle and quality of life (Gurran, 2008). This may well be important in determining where middle-lifers choose to locate, as parttime, flexible employment and entrepreneurial opportunities may become factors in their migration decision-making, as some seaside towns' economic analyses have identified a propensity of part-time, service-orientated work and entrepreneurial spirit (Agarwal, 1999, 2002; Russell and Faulkner, 2004; Russell, 2006b; Beatty et al., 2008; Tallon, 2010). Many companies, particularly those in the service sector, have also begun to recognise that a coastal location is not such a hindrance in light of the provision of modern technologies. This could be good news for seaside towns, where service industries exist second only to public services (Appendix 8). Also, just as places have come to specialise in the kind of job opportunities they offer and the amenities they provide, most have also come to specialise in the stage of life they best fit (Champion 2001, 2005; Agarwal, 2005; Aguiló et al., 2005; Agarwal and Shaw, 
2007; Florida, 2008). This 'New Economy' and socio-economic change (Perrons et al., 2005; Florida, 2010) could see the refocus of human energy towards enabling people to do work that gives their lives real meaning. Locations which can build economic and social systems that can harness full creative capabilities and entrepreneurship could see their urban environments successfully regenerated (Florida, 2010).

\subsection{Key agency and enterprise-based models of analysis}

In the original life cycle model, Butler (1980) acknowledged the importance of the role of entrepreneurs in the 'exploration' stage, albeit their role needs to be given greater recognition as a shaper throughout the lifecycle. Russell and Faulkner $(2004,557)$ recognised this physiological role, where 'one of the main agents of change has been the numerous entrepreneurs who, through a combination of perceptiveness, creativity and the fortuitous confluence of events, are constantly identifying opportunities as they arise and create the organisations to pursue them'. Therefore, while the TALC model provides an overview of resort development and decline stages, it does not capture the feel or reasoning of the destination. It should also be noted, however, that various types of entrepreneurial activity are the result of environmental factors, economic conditions and the individual nature of entrepreneurs. Russell and Faulkner $(2004,563)$ suggest that 'as destinations evolve, so the types of entrepreneurs can also alter'. For instance, 'phase-changing' entrepreneurship can either have a positive or a negative influence on a resort (Agarwal, 1997, 2002; Warnken et al., 2003; Coles and Shaw, 2006; Benedict and Houghton, 2009). This problem can be seen in a number of seaside towns, where business owners have changed the use of or sold their businesses (guesthouses to HMOs, or hotels demolished to make way for retirement homes). If these changes overwhelm infrastructure and statutory arrangements, then the resort's appeal will most likely diminish as a result (Warnken et al., 2003; Russell and Faulkner, 2004). Sometimes entrepreneurial activity, which is portrayed as 'serendipitous entrepreneurship' and is seen as fortuitous, can be the consequence of good timing or just being in the right place at the right time. Russell and Faulkner (2004) recognise that this type of unstructured action happens more frequently in the earlier stages of the 
lifecycle model, whereas 'grand-scale' entrepreneurship tends to involve more decisive actions by an individual or a group that are calculated and more expensive where smaller, less significant independent entrepreneurs sit alongside, providing attractions that are complementary. 'Revitalising' entrepreneurship, on the other hand, transpires when a resort has reached maturity or entered stagnation and occurs when 'a community decides to implement strategies that attract vibrant entrepreneurs who have the vision, the drive, and the cash to revolutionize the market' (Russell and Faulkner, 2004, 564). It is these 'revitalising' entrepreneurs, corporations or key agencies that may be crucial to the reorientation of the English resort. However, for entrepreneurial activity and regeneration to succeed, a supportive culture must be present in the location such that an entrepreneur is motivated to act and feel sufficiently supported to face the challenges (Muller \& Thomas, 2000).

Russell and Faulkner (2004) combined their chaos theory and complexity perspective with Butler's (1980) tourism area life cycle model as an alternative framework, due to its applicability to entrepreneurial behaviour, by illustrating two destinations on Australia's Gold Coast. But those times have changed, with the magnitude of the tourism industry making it more difficult for entrepreneurs acting in isolation to instigate and bring about transformation, as 'corporate' entrepreneurship has come to dominate. Russell and Faulkner $(2004,574)$ recognised that 'with the more professional and planning oriented approach associated with this trend some of the vibrancy and unpredictability of the past has been lost'. They also identified that whereas in the past entrepreneurs managed to circumvent or influence regulating bodies, now corporate entrepreneurship has taken a more measured approach by working with regulators 'to provide a new layer of chaos-dampening influences' (Russell and Faulkner, 2004, 574).

Mueller and Thomas (2000), Russell and Faulkner (2004), Russell (2006b) and Cooper (2006) claim strong leadership and political will are crucial for the reinvention of seaside resorts to take effect. For these authors a local champion is the single, most important success factor. However, the ability of a destination to attract entrepreneurs from outside is often dependent upon the level of entrepreneurship already present in the destination (Russell, 2006b), 
and therefore the statutory key agency leadership role may become ever more important in driving rejuvenation in resorts, if they are to attract entrepreneurs and a regeneration culture.

Ravenscroft and Hadjihambi (2006) use Lamarckian evolutionary theory in socio-economic and cultural regeneration to draw out the importance of planning, development and elected and paid officials' leadership decisions in shaping the environment. They use the framework of Lamarck's theories, in their study of Ayia Napa, Cyprus, to contend that the environment causes changes in the needs of organisms living within that environment. There appears to be tension between the Lamarckian adaptation to the environment (Ravenscroft and Hadjihambi, 2006) along with the predator (physical capital) and prey (natural capital) that shapes the environment (Hernández et al., 2007) and the people that label a destination (either through the indigenous population or in-migration). Human social, cultural and economic capital can shape the personality of places - whether that be through SMTE entrepreneurs who may have changed not only the physical tone but also the social tone of many seaside towns, or migrants in search of work, cheap housing and a place where they can live in retirement; each group can set a direction that may influence the lifecycle curve. Thus, the longer-run issues facing these places have as much to do with their 'psycho-social environment' as with the local business climate and economic structure (Florida, 2008). Seaside town leaders and key agencies must therefore be aware of how their collective personalities can structure the kinds of people and economic activities they can attract, satisfy and retain as people seek out places to live that reflect the characteristics of who they are and who they want to become (Florida, 2008). Furthermore, they need to recognise how their actions can influence the 'meme' within the destination. Migration may well be psychologically motivated in the first place (Rentfrow et al., 2008), so understanding the motives for migration and the destination chosen is a critical part of examination, especially as the twenty-first century suggests a separating out of the 'haves' and 'have-nots' (Bailey et al., 2006; Bailey and Livingstone, 2007; Florida, 2008; Rentfrow et al., 2008), which may ultimately impact on seaside destinations. 


\subsection{Conclusion}

The studies referred to throughout this chapter have highlighted the extent of variations in principal English seaside resort towns. This tends to make any comprehensive analysis of their multifarious interlocking problems of weak labour markets, unbalanced housing provision, high levels of deprivation, population transience, ageing and unstable communities complex and difficult to understand (Beatty et al., 2008; Beatty et al., 2009; Benedict and Houghton, 2009). Authors of studies on seaside resorts, such as Lindley (1973), Walvin (1978) and Walton (1983), evaluated that because urban infrastructure such as promenades, piers, parks, cliff-lifts, etc. were created primarily as the result of investment in Victorian times, this built environment was at the heart of the resorts' problems. Their design and infrastructure has therefore constrained and shaped the paths they have followed. The quantitative statistical analysis, historical legacies and patterns of resort destination lifecycle reveal differing outcomes for resorts, but it is cultural and social phenomena, structural changes and certain key agencies' behaviours that impact these towns equally as hard, though they are far less understood.

Current understandings of seaside resorts reveal they are exposed to many interacting forces that vary in intensity by location: economic and employment choices, housing availability, social, political and cultural forces, historical and physical environments, forces of inertia and the images and labelling of places (Benedict and Houghton, 2009; Walton and Browne, 2010) - all of which render the regeneration strategies of these resorts highly complex.

The meta-structural changes that tend to be viewed as causing the decline of resort destinations resemble a natural evolution in which Butler's (1980) tourism area life cycle (TALC) empirical model has effectively been observed in many seaside resort towns. However, studies by Agarwal (2002, 2007), Agarwal \& Brunt (2006), Coles (2006), Lincolnshire Coastal Action Zone (2006), Shaw and Coles (2007) and the Margate Renewal Partnership (2008) have shown that endogenous factors, such as the behaviour of key agencies and patterns of migration, can reshape the social ecology of resorts and also have a significant impact on resort lifecycles. Although the TALC model emphasises structure 
over agency (Cooper, 1992, 2006), it does not sufficiently consider interdependencies between the exogenous forces that are driving resort evolution and internal agency interaction. Agarwal (2006) argues that resort socio-economic performance is dependent upon the extent and context that key agency interaction has over structure.

While Butler (1980) has never made any claims that his observed TALC model was a predictive model, he nevertheless noted empirically that many resort destinations seem to follow a life pattern. This discussion, as a conceptualised evolutionary model, renders the model as Darwinian in character. The examination of the literature, however, suggests that Lamarck's evolutionary perspective may be better-suited to understanding and managing socioeconomic and cultural regeneration, so much so that the inevitable life/death lifecycle process of seaside resort towns can be managed to avoid this issue, by intervening in and adapting the environment. This management of the lifecycle by key agencies' leadership actions in altering the physical environment and adapting the socio-economic and cultural characteristics of the seaside resort is not well-understood and requires further research. Signifiers in the physical environment, population and image in many seaside resort towns towards retirement purposes have affected the socio-economic and cultural characteristics of these places, though these interconnections are unclear.

This chapter analysed the literature on change in and understanding of principal English seaside resort towns and identified the main strands of theorisation and emerging knowledge gaps. Seaside studies, particularly by those in the 1970s, such as the British Tourist Authority (1975) and Walvin (1978), argued primarily that the Victorian-built environment was at the heart of the problems of seaside decline. Other authors, such as Agarwal $(1995,1997,2002)$ and Cooper (1992, 1997) have examined the action of key agencies and identified them as significant contributors to the direction resorts have taken in the past. However, more latterly, Florida (2004, 2007, 2008), Beatty and Fothergill (2003), the Margate Renewal Study (2008) and Benedict and Houghton (2009) illustrate the role of population in-migration and clustering, particularly retirement migration, and their influence on seaside resort towns, which require further examination. 
The literature review presents a view that over the past few decades, the inmigration of the retired has altered the structure, socio-economic and cultural characteristics and perceptions of many seaside resort towns and contributed to their decline.

The origins, physical and natural environments and history of seaside resorts established a personality, but exogenous and endogenous socio-economic forces, in many instances, have altered them. The historical physical infrastructure and natural environment of seaside resorts can offer both opportunities and constraints and do in fact shape the presentation of seaside towns. However, what does come through a number of seaside studies is that it is the people who shape a town's performance and status. Margate's 'Costa del Dole' and Bexhill-on-Sea's 'Costa Geriatrica' labels are the result of the culture and make-up of the populations who have been attracted to, or reside within, these towns. The literature broadly illustrates this role and contribution of the people in seaside resorts and their influence on a place's personality and socio-economic dynamics. The indigenous population, those who migrate to the location and those who visit all have a powerful influence in this respect (Urry, 1995, 1997, 2002, 2003; Agarwal and Shaw, 2007). It is this issue of middle-life people and places in the context of postmodern society and where they choose to locate or not locate which seem to be a critical ingredient and an area needing examination in the making or undoing of seaside resort towns. 


\section{Chapter 3}

\section{CHARACTERISTICS OF MIDDLE-LIFE MIGRATION AND SYNTHESIS OF FINDINGS}

\subsection{Introduction}

The conventional conceptualisation of retirement migration is problematic, as the literature reveals that retirement migration is becoming increasingly more complex and diverse. Middle-lifers have had life experiences different to those of earlier retirees, as they have lived within a consumer society (Bauman, 1998). Also, with the nature of retirement changing and as work becomes a fundamental part of 'semi-retirement' plans, understanding the motivations of middle-life migration within the context of seaside towns regeneration and the 'New Economy' requires consideration.

Whilst seaside towns have long been recognised as retirement destinations, it has been over the past few decades that retirement migration has supplanted the holiday industry (Stallibrass, 1980; Shaw and Williams, 1997). However, there is recognition that concentrations of ageing populations have had a significant socio-economic impact (European Policies Research Centre, 2006). As Baines et al., $(2004,2006)$ noted, 'the ageing population trend in seaside towns has frequently been regarded as a problem, an increasing burden of decline and dependency for society to cope with; stereotypes of older people as 'takers' rather than 'givers'; social exclusion; and 'isolation' and has led to labels such as Eastbourne's 'God's waiting room” (The Argus, 2005).

The literature review has shown there is gap in knowledge on the interplay between the physical environment, key agency actions and the motivations and behaviours of retirement migration to seaside towns and their impact on the socio-economic and cultural characteristics and the tourism lifecycle. Florida (2008) promotes that people shape the personality of places and through clustering of their collective personalities the socio-economic and cultural characteristics are formed but this is not well understood. 
This chapter therefore analyses conventional retirement migration typologies and highlights the more complex geographical retirement or semi-retirement migration motivations that may influence the future of principal English seaside resorts. From these analyses the chapter concludes by synthesising the findings and knowledge gaps which emerge from this and the previous chapters and addresses the knowledge gaps.

\subsection{Characteristics of middle-lifers}

The twenty-first century British model of lifetime income security is under pressure from a number of sources. The social security, labour and pension policies of the twentieth century became embedded in social structures and became fully institutionalised through contracts (DWP, 2005, 2006; Moen, 2005). However, job security, generous pensions and subsidised private health care are disappearing. Middle-lifers are finding themselves in a changing and challenging world, and it appears that many now do not want to move immediately into permanent retirement; rather, they would like to retire from one job and then shift into something less demanding but which is more meaningful and enjoyable or, as Moen (2005) describes, 'a second act'. Many are seeking to take retirement from high-pressure, time-consuming jobs and take up second, third or fourth careers (Moen, 2003, 2005). Some talk about public service, some shift to less demanding skills that still provide routine and some form of structure, while others may look to start their own business as a way of gaining control over the hours that they work and the effort they put in (AARP, 2003; ELSA, 2008; DWP, 2009a). Phillips (2004) states baby-boomers will not retire on time, as many may need, for financial reasons, to keep working due to health care costs, pension deficits or in order to maintain their standard of living. Studies have shown that 70 per cent of workers born in the baby-boom years expect to work after they retire (AARP, 2002; Hamilton, 2003), and many analysts assume a required income level of about $70-80$ per cent of preretirement income to sustain a lifestyle, which suggests that most will not achieve anywhere near that level, unless they drastically lower their living standards (Philips, 2004; Shackleton, 2004). 
Caro et al., (2000) and Hewlett et al., (2009) argue that the pattern of the human lifecycle is becoming less rigid, and the occupational aspects of the lifecycle will follow suit. Working beyond statutory retirement age is recognised as a fundamental strategy for the UK to reduce social expenditure and financial burden (DWP, 2006). It seems essential that governments make the options for early retirement increasingly more difficult, with more stringent approaches to disability and unemployment (Cameron, 2009; DWP, 2010a, 2010b; Direct Gov, 2010). Accordingly, significant adjustment in the culture and mind-set of retirees and early retirees are required if there is to be support for the implementation of state and management policies. A good example of where these polices are progressing can be seen in Sweden, where retirement is more of a gradual process and revolves around a good partnership between government and employers that has developed over the last two decades. The reason for its success appears to be the availability of part-time jobs, with about a third of workers between 60 and 65 in gradual retirement while benefiting from a partial pension (Delsen and Reday-Mulvey, 1996).

An important equation of this 'demographic ageing' (Dunnell, 2001; Atterton, 2006) is a country's 'total dependency ratio', which considers resources required for the upkeep of living standards for the whole population and is calculated through the ratio of those aged under 16 and those over state pension age, to those aged 16 up to state pension age (DWP, 2009a; ESiF, 2010b). However, the 'economic dependency ratio' is the preferred ratio, as it excludes students aged over 16 , the early retired and the unemployed, and so it takes into account those strictly in work, thereby providing a more meaningful calculation of the country's productive capacity (DWP, 2009a). The 'economic dependency ratio' is also affected by employment rates as well as by numbers in the population of each age throughout the life course. Table 3.1 depicts how there has been an increase in the amount of time spent both in education and in retirement, thus shrinking the time spent in work. 


\section{Time spent in different life stages}

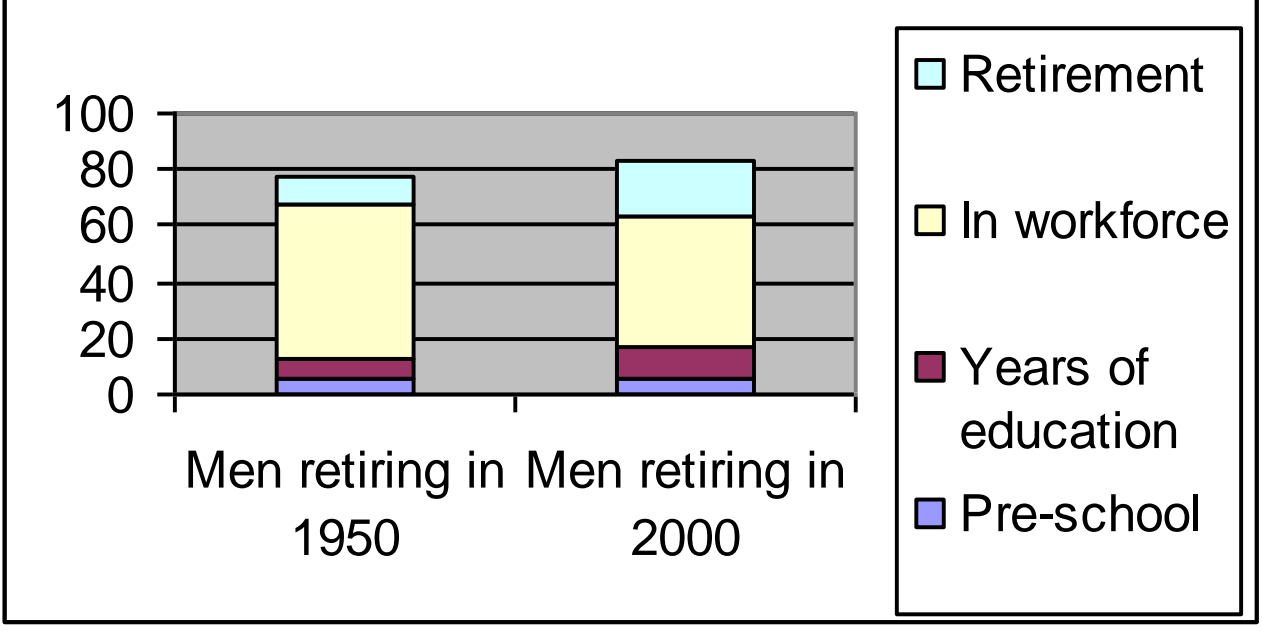

Table: 3.1 Time spent in different life stages

'Source: Department of Work and Pensions (2009a)' (Original in colour)

However, as retirement age becomes less pronounced (DWP, 2009a), previous pre-retirement and retirement models may become less clear (Delsen and Reday-Mulvey, 1996; Atterton, 2006). Also, when middle-lifers leave the labour market, they do not see themselves as entering 'old age' (Evandrou and Falkingham, 2006). The correct age for the onset of 'later life' is considered as 68 for men and 71 for women, whereas a century ago it was 55 and 58 , respectively (ONS, 2010b, 2010c). Many middle-lifers (50-70), above and below pensionable age, wish to remain economically active, whether employed full or part-time, starting their own business, working flexibly or engaging in learning opportunities (DWP, 2005; Hewlett et al., 2009). Middle-lifers also make a significant contribution to the economic well-being of the state through their spending patterns, as 85 per cent of the country's wealth derived from accumulated savings and inheritance (Atterton, 2006) is owned by the over 50s (DWP, 2005), which in the main reaches a pinnacle when people get to their mid-60s, before their wealth begins to decline (Baker and Speakman, 2006). Middle-lifers will have more available combined assets than any previous generation with which to retire, and a considerable number have access to these assets or some form of wealth. Those in the centre of middle-life (aged 60-64), according to DWP (2005), had median financial wealth of $£ 25,000$ in 2003/04 and some of these people will also gain further financial benefits in the form of inheritances (Gunter, 1998; Parry et al., 2004; DWP, 2005; ONS, 2007). 
The DWP (2005) calculate that about three-quarters of those aged 70 and older own their homes, with 83 per cent of these homes being mortgage-free; therefore, middle-lifers are most likely to inherit these properties and consequent wealth. This inheritance may be an important aspect in supporting and sustaining middle-lifers' affluence and might even create a rising dynamic consumer group offering new opportunities for spending, which in turn should help the economy (Johnson et al., 2005; ONS, 2008) and perhaps even help to develop regional economies (European Policies Research Centre, 2006). However, a number of authors in the literature, concentrating on ageing, evidence that pensioner income and distribution are highly imbalanced, and they caution against this view of affluent middle-lifers (Barnes et al., 2004; Parry et al., 2004), as this unevenness is expected to become more pronounced in the future with the emergence of the 'haves' and 'have-nots' in society (Bailey and Livingstone, 2007). This inequality in society is not new, but it could be argued that it is becoming more pronounced and extreme (Bailey et al., 2006; Cetron and Davies, 2010). Put in its most simplistic terms, the shape of socioeconomic profiles has moved from a pyramid shape (upper class at the top working class at the bottom) as we entered the twentieth century to an oval shape (growth of middle class) throughout most of the twentieth century and is now shaping up in the twenty-first century to look more like an egg-timer, with extremes of 'haves' and 'have-nots'.

A study for the Joseph Rowntree Foundation, by Hirsch (2003), concluded that transitions after the age of 50 often represent a crossroads for middle-lifers, from which these individuals will choose or will suit different paths. Whilst the state is unable to control what people at this age will do, they do have at their disposal a number of levers. For instance, the study recommended that to help improve opportunities for those entering middle-life and retirement, employers and society in general should regard middle-life people as a vital resource rather than a growing and inconvenient burden (Hayden et al., 1999). A further study for the Joseph Rowntree Foundation, this time by Godfrey et al., (2004), reinforced the concept of 'duality of ageing'. This characterises ageing as offering new experiences and opportunities at the same time as change and loss. The adaptation that came with changes from moving from work into retirement, and the adjustment that came with ageing, showed considerable 
differences between people and was neither linear nor smooth. These variations were frequently associated with health, resources and capabilities that could be drawn upon in managing the changes (Goldman et al., 1995; Baltes and Carstensen, 1996; Godfrey et al., 2004). Recognition of these differences is important in relation to economic-development and planning strategies undertaken to attract middle-life migrants. Fundamentally, the issue for these communities is that of balancing the positive features of in-migration with the potential negative outcomes, given that many current middle-life migrants are likely to be pre-retirees or retirees seeking changes in lifestyle which are likely to be different from other types of migrants (Rowles and Watkins, 2001; Baines et al., 2004; Bailey et al., 2006). Rowles and Watkins (2001) advocate that middle-life migration can be extensively influenced by an amalgamation of particular migrant types and cohorts entering a location, and that the way in which these migrants' status evolves over their remaining life course can affect the nature of transformations experienced. Therefore, in essence, a more successful economic development strategy may be to attract educated, wealthy middle-lifers in good health, on the basis that they will remain economically active. However, those more reliant on health and social services, and whose health is not so good and who have less wealth, may stimulate economic activity in other ways by increasing demand for services, for which jobs and facilities have to be created (Bauman, 1998; Barnes et al., 2004; Bailey and Livingstone, 2007).

A study by Beatty and Fothergill (2003) of non-employed residents in four seaside towns established that those who took early retirement were more likely to have qualifications such as degrees gained through their life course; this feature of early-retired, middle-life in-migration represented an encouraging aspect for seaside towns, in that this demographic age group could be of use for future economic development. However, Atterton $(2006,71)$ commented that the promotion of middle-life workers needs to be carefully balanced against the needs of younger workers and the need to curtail youth out-migration'. Outmigration may also have an effect on the vitality of the area, and so the retention of young people and the attraction of middle-lifers should form complementary strategies (Rowles and Watkins, 2001; Atterton, 2006). Appendix 9 presents a conceptual model by Rowles and Watkins (2001) that 
summarises the shape of population change and distinctive political, economic and social infrastructure and environmental conditions as seaside towns respond to an influx of middle-life and more elderly migrants. This model shows that the established resident population may be concerned about the impact of the invasion of in-migrants, who may bring with them different views, particularly those of status quo pervasiveness, and so the emergence phase needs to be coupled with a greater need for political involvement. The result of in-migration may be political adjustment as new groups' surface within the local political scene and become more persistent. This changing political environment may result in possible divergence between in-migrants and lifelong residents (Rowles and Watkins, 2001), which has been partially presented in a few studies of seaside towns where the in-migration of different groups and cohorts appears to have led to population and environmental changes (Atterton, 2006; Margate Renewal Partnership, 2008; Benedict and Houghton, 2009; Walton and Browne, 2010).

In times gone by, ageing and retirement were considered to be a period of respectful withdrawal and gradual physical decline, in order to make way for the next generation to take the world forward (Haber and Gratton, 1994). However, evidence is mounting that this expectation of a graceful withdrawal for tomorrow's middle-lifers is that they will probably want to follow a different path (Andrews, 1999; ELSA, 2003, 2006, 2008; Telegraph, 2007, 2010a). As a consequence, market behaviour and policy issues accompanying middle-lifers into old age will be diverse when compared to those faced by the elderly today (Caro et al., 2000; Miles and Miles, 2004). Perceptions seem to matter whereby those who see themselves as younger than their real age are healthier than those who view themselves as being older. Hirsch (2003) also suggested that understanding which comes first - better health or attitude to age - will only be settled as data are gathered from longitudinal studies on this question (ELSA, $2006,2008,2010)$. Also, the consumption lifestyle of baby-boomers and the increasing importance of the relationship between consumption and the urban environment is a complex interaction (Clarke, 2003). For many middle-lifers this may be about finding a location that enables them to 'slowly age' and to maintain their consumer and amenity lifestyle, thus creating a place that is 
young in style but caters for all ages and provides for flexible work opportunities.

These factors are important in determining where middle-lifers choose to locate and cluster - whether with likeminded people (Florida, 2008), to keep them young (Evandrou, 1996; Oc and Tiesdell, 1997; Parry et al., 2004; ODPM, 2006b), or in areas which allow them to maintain their consumer lifestyle through part-time, flexible or self-employment opportunities (Moen, 2005; Hewlett et al., 2009). The migration experience of the middle-lifer, therefore, needs to be viewed within the wider social, cultural, political and economic context of people's entire lives and post-Fordist markets. These post-Fordist markets are not defined by broad social, economic and demographic structures, such as class, age, gender and ethnicity, but by 'lifestyles', 'niche markets' and 'target consumer groups' (Bauman, 1998; Hall, 1998). Jayne (2006) supported Lash and Urry's $(1994,19)$ description that 'consumption is intrinsically linked to quality of life'; hence, as urban places strive (with varying degrees of success) to be consumption places (Lash and Urry, 1994; Miles and Miles, 2004; Miles et al., 2004), the relationships that exist between people and places need to be considered (Featherstone, 1991; Gordon and Goodall, 2000; Jayne, 2006; Mark, 2007). Importantly, consumption can be seen as the link between the individual and the urban environment (Lash and Urry, 1994). The increasing importance of the relationship between consumption and the urban environment is a complex interaction between socio-economic, cultural, political and spatial practices and processes (Clarke, 2003). Bauman (1998) distinguishes between the 'seduced' and the 'repressed' in consumer culture and, in essence, is making a distinction between those who can and those who are less able to enter consumer culture. The 'seduced' are those people in society for whom consumption is in itself liberating, while the 'repressed' are those who do not have access to the required level of resources to the extent that they would like, in order to participate in all that the consumer society has to offer (Bauman, 1998; Miles et al., 2004; Jayne, 2006). This section of the population therefore becomes dependent upon welfare and other state support services and institutions. He concludes that a key feature of consumerism and the contemporary consumer society is division, and not only does the consumer 
society not offer the same opportunities to all, but it also protects those with resources from those without.

Current middle-lifers and future retirees have grown up in this consumer society (Featherstone, 1991; Jayne, 2006) and will have diverse experiences and different attitudes to those of previous retirees (Atterton, 2006). There is now considerable evidence that social class and material factors such as income and occupation affect the health of this cohort and the ability to function in middle-life and into old age (Sweetman, 2003; Parry et al., 2004). Also, people's responses to the changes that have taken effect over time were shaped by their experiences, class disposition and values formed over their life course (Sweetman, 2003; ELSA, 2008). Consequently, as a result of these distinctions and this previous sharp division between 'retirement' and 'work' progressively dissolving, it may be a prerequisite that in order to maintain or improve standards of living in later life, retirement will likely lead to the need to increasingly involve 'income generation'. If this is to be achieved in worthwhile and fulfilling ways, then it may be important for middle-lifers to remain in the region where they have the greatest opportunities and employment-related connections (Florida, 2008). With an ever-increasing amount of middle-lifers beginning to think about 'semi-retirement', this may provide opportunities for seaside towns to enhance economic development. There is also evidence that some social trends could exert more influence over the coming cohorts, for instance partnering arrangements and parent-child relations that may sway local place ties in contrast to the 'conventional' retiree household of the last half century (Longino Jr. and Warnes, 2005). These new types of living arrangements and re-partnering among the middle-life and older age groups are being carefully documented to better understand these changes (ELSA, 2003, 2006, 2008, 2010). For instance, it has been noted that when divorced and widowed older people re-partner, they are progressively more likely to want to retain their own homes. As Johnson $(2005,544)$ describes, "living apart together' may not reduce the months spent in high-amenity retirement areas, but suggests that straightforward migrations could be replaced by seasonal, shuttling and multi-location residential strategies'. Therefore, considering the complex lifestyles of people and living arrangements, the twenty-first century is likely to see more radical developments in pre-retirement and retirement 
migration location preferences and choices than witnessed in the past (Longino Jr. and Warnes, 2005), and it is likely to be evermore difficult to track and illustrate them sufficiently (Johnson, 2005).

This one-way sequence of education, work and then retirement, which appears to be becoming obsolete, once supported a culture and society which no longer exists or is in rapid transformation (Twenge et al., 2010). This is leading to a reappraisal of the life course that may create more opportunities as the Western world experiences more discontinuities than continuities. Changes in family structures and personal lives and in careers and work structures mean that middle-lifers' sense of security and safety, and possibilities for their future, will be challenged (Scott, J., 2000; ELSA, 2008). This is not an environment to which the current elderly retired have been accustomed, as they have enjoyed relative stability over the past five or more decades, so managing change, in whatever manner, may be a real challenge for the leaders and key agencies of seaside resort towns to create the right environment and image and thus attract a change in human social, cultural and economic capital. Therefore, previous regeneration strategies may not be appropriate or will need to adapt to accommodate the shifting nature of the workforce and semi-retired (Cetron and Davies, 2010) and the migration motivations of this cohort.

\subsection{Middle-life mobility}

Retirement migration motivations and geographical mobility are becoming increasingly more varied and complex (Champion, 2001; Longino Jr. and Warnes, 2005; Gurran, 2008; Benson and O'Reilly, 2009a). As identified in the previous chapter, while migration may involve changes in the numbers of people, it also has implications in the composition of the social and cultural structure, which in turn can have important impacts on the economic stability of a local area (Smith and Denholm, 2006), crime and social cohesion (Bailey and Livingston, 2007) and the type of service provision (Travers et al., 2007). Beatty and Fothergill (2004) identified that for seaside towns the single largest flow in the labour market is net in-migration, but that this in-migration is not spread evenly between men and women or across age bands (Appendix 10). Beatty et al., $(2003,39)$ suggest that 'excess in-migration like this would over a 
number of years create imbalances in the local labour market, and in particular lead to unemployment in seaside towns exceeding that in surrounding areas'. The regional location of a seaside resort rather than its size has had some impact on successful adaptation. Seaside resort towns in Wales, the northwest and on the east coast have fared less well in terms of in-migration and employment than the southwest and the southeast. In the southwest this appears to be facilitated by the more robust holiday trade and in the southeast to the wider economic success of the region that spills over into seaside resort towns (Beatty et al., 2008).

Based on their awareness of retirement migration destinations, aside from permanent migration, Longino Jr and Warnes (2005) identified three cyclical patterns of migration. Firstly, 'amenity/seasonal' migration involves middle-lifers who, in general terms, are married, have high levels of previous mobility, tend to be well-educated, affluent and healthy and put less strain on local services. They search for a leisure-orientated lifestyle in locations that have both recreational and service resources (McHugh, 1990; Gurran, 2008). Furthermore, they generally do not relocate permanently but vary their visits, which only end when ill-health or reduced income force a change. Amenity/seasonal migration engender its own types of lifestyle and culture (Williams and Hall, 2002). In contrast is the second cyclical pattern, 'return' migration, of which there are two types: 'provincial' and 'counter-stream'. This involves returning to a location that was previously lived in as part of a life course-related cycle of migration. Provincial return migration tends to involve individuals returning to the communities from which they left when they were younger. These migrants are often pre-retirees in middle-life returning in search of work and in anticipation of retirement. Although they are often less affluent than their amenity migrant counterpart, they tend to be relatively independent, both residentially and financially, and so they are attracted by a lower cost of living (Ackers and Dwyer, 2002). Counter-stream migration refers to the opposite of return flows that develop alongside nearly all major migration streams. It involves retirees who have moved previously to a retirement destination, but later return from whence they came (Rowles and Watkins, 2001). Thirdly, the authors identified 'migrants for assistance'. These are the very old, who move in order to access long-term care as they become frail and 
so require increasing levels of assistance, whether that be closer to family or to service accommodation (Longino Jr and Warnes, 2005).

While permanent retirement migration trends and various other categorisations have emerged under particular historical conditions, such as greater wealth, increased mobility and globalisation, they seem driven by generally established themes encompassed in the descriptions of the rural idyll associated with tourism and travel (Urry, 2002; Baker and Speakman, 2006). The motivations and push-and-pull factors for retirement and middle-life migration fall roughly into four category models (O'Reilly, 2007a): firstly, the 'life course model', where migrants tend to seek climatic and lifestyle amenities, though it is emphasised that age and cohort are important features missing from this model; secondly, the 'migration decision model', which includes adjustment in order to move away from environmental issues such as crime areas, and cost of living or personal resources; thirdly, 'the housing disequilibrium model', where financial motivations related to housing assets dominate the migration decision, and fourthly, 'the place identity model', where moving physically to another location does not always align with moving emotionally - in this instance, Cuba and Hammond (1993) suggest that there are some migrants who remain emotionally tied to their former communities and are unable to put down roots.

Conversely, there are others in search of locations that best represent who they are (Florida, 2004; Florida, 2008; Benson and O'Reilly, 2009a). This point may become more influential, as middle-lifers' migration motivations may alter to include more means migration that reflects their life course and work opportunities. These positive assessments may differ in the years to come as newer middle-lifers' external socio-economic environment alters. As O'Reilly (2007a, 8) states, 'when migrants' experiences fail to match their idyllic expectations they will alter their images and hence the way these places are represented to others. They then try to move on to newer places, in the continuing search for the idyllic lifestyle'. Research shows that most moves are undertaken after careful planning and consideration, with frequently lower housing and living costs as important factors. Whilst initially such benefits are realised, these attractive retirement areas then see the cost of living escalate as 
living expenses and the cost of housing rise while at the same time most of the retired see their income fall (Warnes et al., 1999).

Middle-lifers approaching retirement or in early retirement may choose a particular investment strategy (Rowles and Watkins, 2001) such as 'downsizing', to release capital and thus provide interest-earnings to support their income, or they may seek to avoid various capital gains taxes and so elect to acquire a more expensive home. Whichever investment strategies are adopted, retirement migration is forecast to continue growing as a consequence of advances in telecommunications, as people can keep connected, increased affluence and home ownership, increased longevity, transport and a change from a family-oriented to an individualistic lifestyle (Rees et al., 1996; Rodriguez et al., 1998; Rowles and Watkins, 2001; Fotheringham et al., 2004; Jefferies, 2005). More controversially, it can be argued that predicted growing income inequalities will be a driver for migration in later life, which may also encourage the selection of 'gated' or 'secure' retirement communities (Longino Jr. and Warnes, 2005; Bailey and Livingstone, 2007).

O'Reilly (2007a) and Benson and O'Reilly (2009a, 2009b) suggest that a new form of migration, 'lifestyle migration', is emerging in terms of motivations and moving for a 'better way of life'. O'Reilly (2007a, 2007c) talks about lifestyle migrants, who are identified as being relatively affluent individuals who move temporarily or permanently to locations where the price of property and/or the cost of living is cheaper: 'places which, for various reasons, signify something loosely defined as quality of life' (O'Reilly, 2007a, 1). Where researchers have endeavoured to align their work to wider phenomena by embracing migration concepts such as counter-urbanisation, retirement migration, seasonal migration and amenity migration, King (2002) suggests that moving for a 'better way of life' is uncommon and not covered by these standard migration typologies. It appears that none of these conceptualisations fully grasps the complexity of lifestyle migration in seeking a better way of life. Migration research highlights respondents searching for a better quality of life, in order to make their lives more meaningful - a notion which underlies their migration motivations (Cortazzi, 2001; Benson and O'Reilly, 2009a, 2009b). It is therefore argued by Benson and O'Reilly $(2009 a, 2)$ that 'despite the peculiarity of each 
case, these common lifestyle concerns demonstrate that these different migrations can be considered as a single phenomenon - 'lifestyle migration'.

Benson and O'Reilly (2009b) make an important point, in that the places selected by lifestyle migrants can reveal much about the lives that they aspire to lead and which reflects apparently individual preferences and aspirations (Benson and O'Reilly, 2009b). The idea of a flexible or reflexive habitus (Sweetman, 2003) would suggest that people are now able to select their destinations and the image they wish to reflect in what others have referred to as the 'supermarket of style' (Polhemus, 1995, 91). The value that migrants put on places and how they perceive destinations is subject to a complex interface between previous experiences they may have had of the location and their wider interpretation of the cultural imaginings gained especially through media and images. Seaside resort towns are no exception, and so importantly, the invention and labelling of a place is often based on experience and nostalgia (Shaw and Williams, 1997; Baudrillard, 1998; Scharf et al., 2002; Stevenson, 2003; Godfrey et al., 2004). However, in the absence of any personal experience of a place, a dominant impression is imposed, and for a number of south coast resorts they are perceived or referred to as 'Costa Geriatrica'. This process is a significant factor contributing to the development of positive or negative images of a place, including stereotyping and stigmatising (Stevenson, 2003). As such, when a place acquires a 'name', this 'name' unifies and sets it apart (Jayne, 2006). Also, middle-lifers' individual circumstances, including their education, class disposition and economic capital, as well as certain historical and material conditions at the point of migration, all contribute to how they perceive a place. Thus, middle-life migration and future employment and amenity decisions will likely require locations offering job opportunities, thereby encouraging the clustering of cohorts of like-minded people and providing amenities that presents 'a young town for all ages'. Coles and Timothy (2004, 3) state through their work on conceptualising diasporas that 'groups of people are scattered across the world but drawn together as a community by their actual (and in some cases perceived) common bonds of ethnicity, culture, religion, national identity and sometimes, race'. Therefore, the selection of a place is the result of both individual agency and structural constraints and their reflexive habitus (Hochschild, 1983; Bourdieu, 1984, 1990; Hetherington, 1998; 
Sweetman, 2003; Coles and Timothy, 2004) closely associated with variables such as class and ethnicity but becoming increasingly fragile as this standardisation is dismantled through consumerism and how people go about constructing their own identities (Giddens, 1991; Maffesoli, 1996; Sweetman, 2003). Certain forms of habitus may therefore be inherently reflexive, while the 'flexible or reflexive habitus may be both increasingly common and increasingly significant due to various social and cultural shifts' (Sweetman 2003: 529).

Recognition of the diverse nature of this retirement migration has important implications for local authorities with regards to the economic development strategies they may adopt (Rowles and Watkins, 2001; Gurran, 2008). However, with the government changing pension arrangements and the retirement age (if in future a retirement age exists) (DWP, 2003b, 2006, 2010a, 2010b; Pensions Commission 2004; Directgov, 2010), the behaviours and attitudes of middle-lifers towards the traditional retirement ethos is shifting (Champion, 2005). Therefore, location is becoming even more important in the future as baby-boomers enter middle life and seek a form of retirement that enables them to maintain their consumer and amenity lifestyles while at the same time being provided with opportunities for work or the chance to start a new business. This has important implications for seaside resorts that currently have high numbers of the retired and elderly living in their area and where work has not been a requirement for previous retirement living arrangements.

The literature suggests that retirement will be far more complex than the current situation (Longino Jr. and Warnes, 2005; DWP, 2009a; Cetron and Davies, 2010) and require more vibrant and dynamic economic places to reside, which seaside retirement towns do not currently offer. What the literature shows is if key agencies and administrations can attract wealth creators, this appears to be a way of reversing decline and stagnation (Smith, N., 2000, 2002; Smith, M.K., 2003, 2007; Lees, 2003b, 2008; Smith and Denholm, 2006; Lees et al., 2008; Tallon, 2010). However, not all middle-lifers are healthy or wealthy (Glaser and Grundy, 2002), and so the task may be to identify the clustering of the 'right' people and understand what motivates them, in order to inform key agencies how the physical environment could be altered. However, further research is required into migration motivations in order to understand what might need to 
change in the physical environment to attract wealthier, healthier middle-lifers (Scott, A. J., 2000; Clarke, 2003; Lancaster and Stillman, 2003; Powe and Shaw, 2004).

\subsection{Synthesis of findings}

The analysis of the principal English seaside resorts' have shown their fortunes and tourism structures changed over the past half a century, that has altered the towns' physical environments and inhabitants. The in-migration of the retired that took over from the holiday industry in many of these places changed their socio-economic and cultural structures. Created by exogenous forces, where it has previously been argued that the 'TALC refers to resorts that carry with them the potential seeds of their own destruction, as they allow themselves to lose their original qualities which attracted visitors' (Plog 1974, 58), the changes (as discussed in this and the early chapters) have also been fuelled by endogenous actions influenced by key agencies decisions. Interventions in the physical environment by key agencies have been a catalyst for change and have encouraged the in-migration of groups of people including the retired. So, as far as seaside town resort development is concerned, it may be argued that the inevitable time-mediated life/death lifecycle process is more the result of a complex series of key agency decisions, thus resembling a Lamarckian evolutionary construct where physiological interventions changes the socioeconomic and cultural conditions (Ravenscroft and Hadjihambi, 2006; Hodgson, 2001).

For many seaside town historians Shaw and Williams (1997), Walton (2000), Watten (2000), however, they have presented a picture resembling a Darwinian evolutionary perspective, whereby they follow Butler's TALC model of innovation, growth, maturity and decline but where some resorts survive and others fail depicting 'survival of the fittest'. For Darwin the evolutionary development was a function of two complimentary constructs; random variation and random selection. Darwin argued that improvements in species occur randomly with only the most suitably adapted being able to survive (Packard, 2007). The Darwinian evolutionary construct involves detailed, causal, step by step, understanding of evolution based on the features of variation, inheritance 
and selection whereby, the inheritance of genotypic instructions by individual units, a variation of genotypes, and a process of selection of the consistent phenotypes according to their fitness to their environment (Hodgson, 2001). However, the development of many resorts appears to be usually regulated by plan to ensure selection is anything but random. The ability of planners to influence development of resorts suggests an interventionist's non-random evolutionary model typical of Lamarck's ideas on species change (Ravenscroft and Hadjihambi, 2006; Mehmood, 2010). Thus some literature argues that just as a decision to develop a resort is neither random nor the result of natural selection, so its subsequent life and eventual decline is similarly a result of key agency intervention (Cooper 1992; Hayward 1998) that is more akin to Lamarckian evolutionary perspectives.

Authors such as, Metcalfe (1993), Laland et al., (2000) support theories of social and biological evolution where a version of Lamarckism at the social sphere can be consistent with Darwinian principles in which they argue that they must be dependable with each other but do not have to be identical. The Lamarckian socio-economic and cultural evolutionary constructs notes the importance of 'the influence of the environment'. Essentially, Lamarck's notion of evolution is driven by environmental changes rather than by genetic variety within a population. In Lamarckism, organisms adjust continuously as if attempting to reach harmony with their environment. Lamarckism is primarily associated with the proposition that acquired characteristics can be inherited and influenced by the environment (Hodgson, 2001, 2005). On this point the contrast with Darwin is clear. Lamarck argued that variation was a function of the environment, but for Darwin 'variation was present first, and the ordering activity of the environment (natural selection) followed afterwards' (Mayr, 1982, 354). For Lamarck, the environment was the key agent of change. In contrast, Darwin developed a view that intergenerational change resulted from a combination of renewed variation and environmental selection. For Darwin, variety is the evolutionary fuel.

There is considerable debate as to whether socio-economic and cultural evolution is therefore, Lamarckian or Darwinian in character. However, the literature presents a view that socio-economic and cultural evolution can be 
both Darwinian and Lamarckian and that they may dovetail together (Hodgson, 2001). However, in Lamarck's evolutionary construct there appears to be a propensity to inherit improvements rather than impairments but there is nothing that in principle rules out the imitation of detrimental behaviours and this may also contribute to the decline of resorts. There are echoes of both Darwinian and Lamarckian theory evident in the literature of evolution in which Gould (1990) and Steele et al (1998) suggest this position is defensible in which the external environment is understood in relation to Darwinian selection, while changes to the local built environment are mediated according to Lamarckian inherited traits where variations occur through adaptations to the local environment.

The research literature suggests that as the physical environment changes, so the in-migration of populations that are attracted to this changing environment influence the character of the seaside town and in turn its socio-economic performance (Beatty et al., 2003; Beatty and Fothergill, 2007; Shaw and Coles, 2007; Beatty et al., 2009). It is evident from the literature review that the exogenous forces at work have had a profound effect on resorts, though equally, endogenous actions and the agency influence at a local level are critical in determining the meme that produces place-specific outcomes that help adapt a resort's purpose and image. The Lamarckian interventionist perspective, according to Ravenscroft and Hadjihambi (2006), is therefore created by key agencies' actions rather than natural evolution. This disputes the historical predictability of the lifecycle model and instead situates resort survival to adaptation (Ravenscroft and Hadjihambi, 2006). This outlook is grounded in key agencies' leadership capabilities and the ability to cultivate support for physical regeneration and improvements to the human-made and built environment, which allows it to adapt to and alter human social, cultural and economic capital. This seems to be a critical point of examination.

This and the previous chapters have identified that both agency and structure have an influence over the human, social, cultural and economic capital in seaside towns and that the people have influenced the personality of places, their physical and social tone and associated images and labels (Agarwal, 1999, 2002, 2007; Zimon, 2000; Hernandez et al., 2007; Florida, 2008). For 
instance, seaside towns with a high preponderance of the elderly are prevented from regenerating as a result of their residents' apparent resistance to change (Walton and Browne, 2010), which in turn creates an image of 'pensioner ghettos and a grim future for younger workers' (Telegraph, 2010b), whereas those neighbourhoods that have been the focus of modification and gentrification experience a transformation in their socio-economic, cultural and environmental character (Pacione, 2005), where social displacement and/or social upgrading occurs (Phillips, 2002).

Coles and Hall (2006) suggest that place, people, image and mobility cannot be separated; therefore, some view seaside town deprivation as a problem, while others may see such areas of decline as an opportunity (Smith, 1996; Cameron, 2003; Clarke, 2003; Lancaster and Stillman, 2003; Powe and Hart, 2009). The 'New Economy' (Florida, 2010) presents how people will reconfigure their lives and where the baby-boomer generation has dominated for most of the past four decades (Shackelton, 2004; Evandrou and Falkingham, 2006; Cetron and Davies, 2010) and is where change in seaside towns needs further examination. Crewe (2000) and Champion (2001) suggest that places are not just being presented as centres of economic activity but equally importantly as culturally vibrant places where contemporary consumption is intrinsically linked to quality of life and as places in which to live and work - a feature which may be an important factor in middle-lifers' migration motivations. The work by Ravenscroft and Gilchrist (2009), looking at artists living in Hastings, implies that the cultural ecological draw to Hastings of Bohemians and artists may be more to do with location and their 'address'. Therefore, images and labels that define places are equally strong in setting the direction of a location.

Middle-lifers are finding themselves in a changing world and authors on the ageing population predict that many baby-boomers will continue working into retirement age as occupational lifecycle becomes less rigid. Middle-lifers of today are distinctly different from those of the past (Godfrey et al., 2004), having grown up with different life experiences and being the generation that drove the consumption society (Clarke, 2003), so their location choices will be influenced by their perceptions, employment opportunities, amenities, consumption, housing, transport, aesthetics and clustering opportunities, in order to meet their 
demands and expectations as they move into their next life stage (Atterton, 2006; Croucher, 2006). However, evidence is mounting that there will be a growing disparity in pensioner income and distribution that according to Florida (2010) and Champion (2005) will influence where the most skilled and educated live. It may be important for middle-lifers to remain in the region where they have the greatest opportunities and employment-related connections but there is insufficient understanding of the motivations behind the choice of seaside retirement living that may have future consequences for seaside towns.

\subsection{Introducing the focus of examination}

Early seaside studies argued that it was the Victorian-built environment that was a contributory cause of decline of resorts. More latterly other authors cited key agencies as significant contributors to the direction of the seaside town. However, others are illustrating the role of population in-migration and clustering as an influence on seaside resorts. The literature review in this and the previous chapters has identified a gap in knowledge about seaside retirement towns and the in-migration of the retired, the interconnections between the physical environment, key agency actions and subsequent impact on the socioeconomic and cultural outcomes. The area identified for further research, through which to develop a better understanding of seaside regeneration, requires analysing the past actions of key agencies that have contributed to the changes and how they perceive their collective actions shape the physical environment and the kinds of economic activities and people they can attract that influences the effects of these changes in a seaside town.

The research problem identified from the gap in the literature is that there is insufficient understanding of retirement migration into principal English seaside resort towns. Where O'Reilly and Benson (2009a) suggest that 'lifestyle migration' as an emerging theme in terms of motivations for moving that entails a search for a 'better way of life' and to 'lead out a new life' in order to make their lives more meaningful there is a gap in knowledge and a need for a better understanding of the migration motivations of middle-lifers resort retirement. The selections of a seaside town in which middle-lifers may migrate involve identifying not only the influence of economic factors but also the effect of 
lifestyle patterns and aspirations that could affect this destination of choice and clustering. Florida (2008) contends while opposites do attract, most people are more content with places that match, but this requires further examination.

Related to this are the perceptions and images of a place held by middle-lifers when making migration decisions and the interconnections between the physical environment, key agencies and middle-life migration.

The literature analysis of the principal English seaside resorts has revealed that a detailed study of the behaviours, values and motivations of middle-lifers entering retirement and key agency actions in a typical seaside town will shed light on the interrelations and interconnections between firstly the processes of change in the physical environment (human-made built and urban), secondly the key agencies that seek to affect change and finally the effects of middle-life migration (aged 50-70 years) and the perceptions generated from these changes. The literature suggests retirement migration is becoming increasingly complex. Seaside retirement resort towns today may need to develop into more vibrant and dynamic places if they are to attract baby-boomers who have lived through a consumer age and who have different life experiences and attitudes to previous retirees. This proposition demands better understanding.

The next chapter seeks to identify what research strategy, methodology and methods will best suit this empirical study. 


\section{Chapter 4}

\section{RESEARCH METHODOLOGY}

\subsection{Introduction - theoretical influences and methods}

The aim of this research is to examine socio-economic and cultural change and regeneration in a seaside town to understand the interconnections between processes of change in the physical (human-made built and urban) environment, key agencies that influence change and the effects of middle-lifer migration (aged 50-70 years of age).

The thesis addresses the context of middle-lifer retirement migration and takes an evolutionary perspective. In particular, the research explores not only the actions of key agencies but also the role of middle-lifer in-migrants as potential key actors shaping the town

The literature review in Chapters 2 and 3 presented a discussion of an application of Lamarckian theory, whereby changes in the economic and social structures of seaside resorts were conceived as resulting from a complex series of changes in the physical environment linked to planning and development decisions by key agencies rather than as a life cycle process (Ravenscroft and Hadjihambi, 2006), as outlined in Butler's TALC model of change in resorts (Butler, 2005a, 2005b; Berry, 2006). By taking a Lamarckian socio-economic and cultural evolutionary approach, if key agencies intervene in a strategic way in the physical environment, then this may adapt the nature of the populations living and visiting seaside resort towns which may address some of the stagnation and decline. The ability of key agencies to develop a series of improvements to the physical environment (the built and urban environment), and to alter the nature of the demographics, has been identified as a critical focus for examination in understanding change in local economies (Russell and Faulkner, 2004; Tallon, 2010).

The literature review identified a gap in knowledge that as the physical environment changes, so the in-migration of retired populations that are 
attracted to this changing environment alters, which ultimately can influence the character and personality of a seaside town and in turn its demographics and socio-economic performance (Agarwal, 1995, 1997, 1999a, 1999b, 2002, 2007; Beatty and Fothergill, 2004, 2007; Beatty et al., 2008). However, a better understanding of migration motivations of middle-life retirees might be acquired through an analysis of physical changes and economic factors, but also the effect of lifestyle patterns and aspirations that could affect their destination of choice. Related to these issues is the need to explore the perceptions and images of a place held by middle-lifers and how these elements might reflect their personality and the 'psycho-social environment' (Erikson, 1987) they are seeking when making migration decisions.

A mix of research methods will achieve a better understanding of these issues, revealing a complex set of related processes. Crotty (2003) sets out two key issues in the research design process: the choice of methodology and methods and the arguments that justify this choice. He proposed four questions that touch upon what he claimed to be the four basic elements of research: firstly, what method is proposed; secondly, what methodology oversees the selection and use of methods; thirdly, what theoretical perspective determines the methodology in question and fourthly, what epistemology guides the theoretical perspective. These four questions are used to underpin the structure of arguments in this chapter, which outlines how the epistemology, theory and choice of methods are related to the research undertaken in Bexhill-on-Sea.

The research methodology is therefore set out in two parts. Part one discusses interactions between the methodology and the theoretical perspective and contains three subsections. The first discusses the theoretical perspectives underlining the research design, the second includes a discussion on related epistemological issues shaping the choice of research methods and discourse analysis and the third subsection outlines choices concerning the adoption of a single-case study as part of the research design. Part two considers the details of the research design and the method used, and it also discusses the nature of the respondents and their recruitment. The second part of the chapter also presents arguments concerning reflexivity, ethics and bias, thereby highlighting 
how the research design ensures the validity, authenticity and credibility of the findings.

\section{1 (Part 1) - Methodology}

\subsubsection{Theoretical and epistemological issues}

For the purposes of formulating the choice of an appropriate research design, methods and methodology, a theoretical perspective is discussed.

Quantitative research usually entails taking a deductive approach where the intent is on testing theories in which practices found in the natural sciences and of positivism are incorporated (Brewer, 2000; Potter, 2006). It represents a view of social reality as an external, objective reality (Silverman, 2010). By comparison, qualitative research and post-structuralism usually accentuate words in the collection and analysis of data, rather than quantification. It principally accentuates an inductive approach, in which the emphasis is on the production of theories, and it precludes approaches of the natural scientific model and of positivism in particular, in favour of the prominence with which individuals understand and interpret their social world (Silverman, 2010). Qualitative research also expresses a view of social reality as a continuously fluctuating emergent property of an individual's creation (Silverman, 2005, 2006). Therefore, the study of social sciences may require a different research procedure, one that portrays the uniqueness of humans as opposed to a supposed natural order (Von Wright, 1971).

The qualitative research literature expresses the essence of the qualitative and quantitative divide by claiming that quantitative research differs from its counterpart, in that it is essentially nomothetic (Bryman, 2004). In short, it displays a deterministic outlook entailing, to a greater extent, 'measurement and analysis of causal relationships between variables' (Denzin and Lincoln, 2000, 8). Quantitative research, it is argued, privileges the quantification, prediction, formulaic precision, verification and falsification of a priori hypotheses or theories, in the place of analysing complex social qualities, meanings and processes (Flick, 2007). Most qualitative researchers, however, accentuate a 
preference for the theory as something which in part surfaces from the collection and analysis of data (Bryman, 2004).

Qualitative research has also been influenced by postmodern perspectives of the social world, which is observed as a context from which various interpretations can be made. Therefore, 'knowledge' of the social world is relative, and any account is just one of many possible ways of rendering social reality, so 'postmodernists offer 'readings' not 'observations'; 'interpretations' not "findings" (Rosenau, 1992, 8). Postmodern writers are cautious about the knowledge claims made when findings are reported, and they adopt an approach of exploring the bases and forms of these knowledge claims (Denzin and Lincoln, 2000; Hammersley and Atkinson, 2007). Furthermore, postmodernists recognise such ethnographic accounts of their texts and their authority to represent a reality as problematic, because 'there can never be a final, accurate representation of what was meant or said, only different textual representations of different experiences' (Denzin and Lincoln, 2000, 267). Such a view, however, can render as challenging the very idea of what social scientific knowledge is or in fact comprises.

There are, however, conflicting views regarding the social sciences underpinning debates on quantitative and qualitative methods. The positivist view is that natural and social sciences are essentially the same and each is concerned with discovering natural and universal laws that regulate and determine individual behaviour (Dwyer and Limb, 2001; Bryman, 2006; Creswell, 2007). Another view, while sharing the same desire for rigour, accentuates how individuals differ from each other and from inanimate natural phenomena, in which case the justification of laws is not appropriate (Denzin and Lincoln; 2005 Bryman, 2006). These two views of social science are underpinned by different explicit and implicit assumptions. The first concern assumptions of an ontological kind (Cohen and Manion, 2004) in relation to the very nature or essence of the social phenomena being investigated and whether social reality is external to individuals or the product of an individual's consciousness (Crotty, 2003). The second set of assumptions is epistemological in nature. These concern the basis of knowledge - its nature and forms, how it can be acquired and how it is communicated to other human 
beings (Cohen and Mansion, 2007). Cohen and Mansion $(2007,6)$ also suggest that there are views of 'knowledge as 'hard', real, objective and capable of transmission but also arguments that knowledge is 'soft', more subjective, spiritual or even transcendental being based on experience and insight of a unique and essentially personal nature'. Where the researcher positions themselves in this particular debate will affect how uncovering knowledge on social behaviour is undertaken. The interpretation that knowledge is 'hard' will require researchers to align more with the methods of natural science, whereas seeing knowledge as 'softer' implies a rejection of the ways of natural science.

Bryman (2004) argues that qualitative research involves an interpretive and a humanist approach to the world and that the researcher should immerse themselves in the study of things in their natural settings, thereby attempting to make sense of, or interpret, phenomena. Qualitative research tends to dispel methodological tenets that entail the isolation of phenomena from their natural spatial and temporal settings and promotes depth of inquiry through contextual specificity and situational complexities (Denzin and Lincoln, 2008). However, Charmaz $(2000,511)$ argues that 'qualitative data can and should have an important role in relation to testing of theories and there is no reason why qualitative research cannot be employed to test theories that are specified in advance of data collected'. The testing of theories in the course of the research process between testing emerging theories and collecting data is predominately a feature of the grounded theory approach (Charmaz, 2000). Qualitative research therefore contains various potentially conflicting models and illustrates the quantitative/qualitative dichotomy. As such, it is argued that polarisation in social sciences should be avoided and instead qualitative research must be rigorous and authentic and critical standards maintained (Bryman, 2004; Silverman, 2009, 2010)

Many qualitative researchers are opposed to research that entails the imposition of predetermined formats on the social world, but by adopting a structured method of data collection, it is argued that the researcher has already made certain judgments about the nature of the social reality that is to be encountered (Silverman, 2009). Therefore, the researcher is constrained in the way in which they can genuinely adopt an impartial view of those being studied. 
As a result, qualitative research tends to be a strategy that tries not to delimit areas of inquiry too much and sets out to ask general rather than specific research questions (Bryman, 2006; Silverman, 2009). Therefore, the validity of qualitative research is usually evaluated by the criteria and principles of 'trustworthiness', 'credibility' and 'authenticity' (Flick, 2006) as opposed to evaluation principles that are traditionally associated with the quantitative paradigm (i.e. reliability and generalisability) (Denzin and Lincoln, 2005). 'Authenticity' and 'credibility' can be achieved by recording a thorough and detailed account of the research process (Silverman, 2001; Guba and Lincoln, 2004; Denzin and Lincoln, 2005) and should include using appropriate research methods, clearly outlined criteria, sensitivity of the methods used to address the research question and subject, accepted procedures, systematic analysis, adequate discussion and a clear distinction between data and interpretation (Silverman, 2006). Consequently, 'authenticity' is often a key criterion for evaluating qualitative research rather than 'reliability', as the aim in qualitative research is usually to gather an authentic understanding of people's experiences (Silverman, 2001; Denzin and Lincoln, 2005).

This thesis emphasises the effect on local socio-economic and cultural structures of the physical environment, key agencies, individual experience and perceptions, which subsequently influenced the choice of research methods. Understanding middle-life migration motivations, and the search for a particular lifestyle and the impact this may or may not have on the environment of the destination, required a research approach that understood the migrants' emotions and motivations. The research aims also require an understanding of values, relationships, practices and norms in the public, business and community sectors - issues well-suited to qualitative research methods, especially those involving narrative inquiry (Clandinin, 2007: Holstein and Gubrium, 2008) where 'experience is understood narratively' (Clandinin and Huber, 2010, 1). As a result, and as discussed in more detail below, an emotionalist narrative-based research design was adopted that was influenced by post-structuralism and related theorisations of discourse analysis. In particular, this research design was adapted to the specific exploration in this thesis of discourses concerning the effect on local economic, cultural and social change of the interconnections between alterations in the physical environment 
(in this instance meaning the urban and built environment as opposed to the natural environment), actions by key agencies and individuals and the nature of middle-life migration to a seaside town. The next subsection explains the reason for adopting this focus on a narrative discourse-based qualitative research design and how this was then influenced by certain epistemological considerations.

\subsubsection{Research methodology and narrative inquiry}

This section provides the rationale behind the choice of the research methods that underpin the use of narrative inquiry. The two research methods adopted, primarily interviews but also documentary analysis to inform the findings, will be discussed later in the section. Narrative inquiry is seen as the most appropriate overall approach for generating and analysing data from which to gain an understanding of the complexities of this research rather than discourse analysis, but the data collection and analysis are also influenced by certain key features of discourse analysis. While these two methodologies have overlapping similarities and are appropriate for this research, narrative inquiry is favoured. Both approaches analyse texts in an attempt to uncover meanings in what people say (in this case, interviews) and which in a way seek to take account of the social, political and cultural contexts. However, discourse analysis tends to either focus on language used or, in the case of Foucauldian discourse analysis, on the analysis of power and power relations (Willig, 2001). Discourse analysis is not simply a research methodology - it provides an alternative way of conceptualising language and involves a theoretical way of understanding the nature of discourse and the nature of psychological phenomena (Willig, 2001). Discourse analysts 'argue that when people state a belief or express an opinion, they are taking part in a conversation which has a purpose and in which all participants have a stake', whereby 'to make sense of what people say, we need to take into account the social context in which they speak' (Willig, 2003, 161). Also, Willig $(2001,89)$ claims 'that the world can be read in a number of ways and that, far from giving rise to mental representations, objects and events are, in fact, constructed through language itself... If language constructs, rather than represents, social reality, it follows that there can be no objective perception of reality'. Thus, what differentiate 
people are not their attitudes or explanations concerning an object or event, but rather the way in which they construct the object or event (Potter, 1997; Willig, 2001).

Narrative inquiry, by contrast, focuses on understanding people's lives through their stories and less on power. This research does not seek to analyse either language or power; rather, it analyses people's stories. Narrative inquiry follows a 'recursive, reflexive process of moving from field (with starting points in telling or living of stories) to field texts (data) to interim and final research texts' (Clandinin and Huber, 2010, 1). In this study, two sets of 'stories' are analysed. The first are key agencies' narratives of how they perceive Bexhill-on-Sea has changed and how they believe regeneration has affected this change. Then there are the middle-lifer stories regarding their lives and why they moved to Bexhill-on-Sea. As such, this narrative inquiry research adopts a thematic analysis that tends to draw on theory and previous research to identify themes, as opposed to grounded theory, which allows themes to emerge from the data (Charmaz, 2000). Therefore, the purpose of approaching an understanding of the research aims and objectives through a narrative inquiry perspective is to develop a view of social reality from the point of view of people's verbal expressions of their beliefs, emotions and attitudes about cognitions that reside in their minds. To make sense of what people say, the social context in which they speak also needs to be taken into account, and in this sense the methodology is influenced by discourse analysis (Willig, 2001; Clandinin, 2007). Narrative inquiry, however, is not simply a research methodology - it provides a theoretical way of understanding the nature of discourse, emotion and psychological phenomena that are the focus of the interviews with key actors and middle-lifer migrants (Potter, 1997; Willig, 2001; Clandinin, 2007).

Proponents of narrative inquiry also argue that account must be taken of the experiences of the researcher in the process of designing, studying and engaging in enquiries (Clandinin, 2007). There are a number of ways to study the world, and the interactions of people and narrative inquiry recognises the interactive quality of the researcher-researched relationship, primarily using stories as sources for data analysis, and then understanding the way in which what is known is embedded in a particular context and that narrative knowing is 
essential to the inquiry (Clandinin and Connelly, 2000; Clandinin, 2007). For the purposes of this research, the researcher adopted a narrative inquiry that also included taking account of the relationship between researchers and researched, using words as data and with a focus not on the universal and general but more so on the local and specific (Clandinin and Connelly, 2000).

Narrative inquiry also requires a shift away from the objective researcherresearched relationship, which has been influential in physical and social sciences, towards a more relational view involving a reconceptualisation of the status of the researched in the relationship (Clandinin, 2007). It was recognised by the researcher, therefore, that the research subjects in Bexhill-on-Sea were not bound, static or decontextualised. Humans and their interactions were embedded in context, and the approach adopted to analyse the data discussed below sought to take account of how this might affect the findings in the long run (Polkinghorne, 1988; Silverman, 2006; Clandinin, 2007).

In choosing an appropriate research method for a narrative inquiry approach, it is recognised that there are no right or wrong methods; there are only methods that are appropriate for the research topic (Gubrium and Holstein, 1997). Four major methods are commonly used by qualitative researchers to examine narratives and discourses (Silverman, 2001): observational studies, which are often chosen as the method for understanding another culture; texts and documents, where researchers analyse material through categorisation, in order to enable coders to arrive sufficiently at similar results; video recordings, which are becoming an increasingly important part of qualitative research, as compared to field notes they can provide a record of 'naturally occurring' interactions, and interviews, which can involve 'fixed-choice' or 'open-ended' questions.

The interview process was the prominent method for gathering empirical data, and the specific details of sampling and respondent characteristics will be discussed in more detail later in the chapter. The use of documentary analysis was also adopted in the research, albeit not for purposes of triangulation, where case study facts or events are reinforced by other sources of evidence and then converge (Yin, 2009). In this thesis, documentary evidence was used for 
informing the findings taken from the in-depth interviews, especially with key actors. A third area considered as a means of informing the findings was that of observational studies through the researcher being present at key agency meetings. However, this approach was discounted, as the researcher was constrained in this respect due to their employment role within the local authority and their close participation in some of the meetings.

The participant interviews took place largely within their own settings, such as home or office, rather than in systemised, unnatural and fixed timeframe circumstances, in order to avoid imposing artificial structures upon the participants and the data. Their chosen settings and the in-depth interviews were used to try to gain an insight into complex narratives, stories and emotions that needed to be understood as part of the narrative inquiry approach. Other qualitative methodologies such as observation or documentary analysis were unlikely to gain an insight into the emotions that underpinned the narratives (Hammersley and Atkinson, 2007). Furthermore, it is argued that an understanding of the impact of social and cultural context upon representations can be better investigated through research into the participant's own setting, as entailed in this study (Creswell, 2007).

The knowledge gaps required an epistemological approach that considered understanding the values, practices and norms of key agencies as well as the nature of middle-lifers' migration emotions and motivations. This best suited the adoption of interviews requiring a 'softer', more subjective angle, thus allowing an insight into issues of a unique and personal nature. The interviews also enabled the researcher to consider through interview reflections and field notes the influence of the researcher-researched relationship.

Once interview data has been collected, there are three main approaches adopted by social scientists to analyse said data. First, following a more positivist perspective, is the approach that takes the view that the data collected from interviews give access to 'facts' and 'truths' about the world (Silverman, 2009), with the primary goal being to generate data that are both valid and reliable (Creswell, 2007; Silverman 2009). The main ways to achieve this are through the random selection of interview samples and the administration of 
standardised questions with multiple-choice answers which can be organised and tabulated (Silverman, 2001). The second approach, argued for by those advocating a constructionist approach, sees interviewers and interviewees as always actively engaged in constructing meaning (Webster and Mertova, 2007). Silverman $(2006,118)$ states that 'rather than treat this as standing in the way of accurate depictions of 'facts' or 'experiences', how meaning is mutually constructed becomes the researcher's topic'. What distinguishes constructionalists is 'focused interaction' as a topic in its own right, but criticisms of this approach are that there is a danger of focusing on conversational skills rather than on the content of what interviewees are saying and its relation to the world outside of the interview (Silverman, 2001, 2009, 2010; Webster and Mertova, 2007). The third approach, according to those coming from an emotionalist perspective, sees interviewees as 'experiencing' subjects where generating data aims to give an authentic insight into people's experiences and how they actively construct their social world. The main research method employed to achieve this is through unstructured, open-ended interviews sometimes based upon prior participant observation (Creswell, 2007; Silverman 2009). As Silverman $(2006,123)$ stated, 'Emotionalists' concern is not with obtaining 'objective facts', but with eliciting authentic accounts of subjective experience', and so in order to achieve this aim, emotionalists believe that interviewers should try to formulate questions and provide an atmosphere conducive to open and undistorted communication (Holstein and Gubrium, 2008). However, there are issues that need to be addressed with this type of approach, in that interviewees - if they have a lack of commitment to the issues being researched - may fabricate 'facts' or interact in a different way (Silverman, 2006; Webster and Mertova, 2007). The difficultly involved in penetrating private worlds means that researchers need to consider the relative status of the interviewer and the interviewee and the context of the interview (e.g. at home, work or somewhere selected), to avoid misunderstandings between the interviewer and the interviewee (Silverman, 2001).

Much of the data gathering and analysis processes will adopt an emotionalist narrative inquiry approach where, as Webster and Mertova $(2007,3)$ comment, 'Narrative is not an objective reconstruction of life; it is a rendition of how life is perceived'. As such, it is based on the respondent's life experiences and 
involves chosen parts of their lives. As Webster and Mertova $(2007,4)$ go on to explain, 'It requires going beyond the use of narrative as rhetorical structure, to an analytical examination of the underlying insights and assumptions that the story illustrates'. As the story unfolds, the approach to data analysis has to allow the complexities of characters, relationships and settings to be explored (Webster and Mertova, 2007). Narrative research, therefore, does not endeavour to create conclusions of certainty; rather, its findings aim to be 'wellgrounded' and 'supportable', retaining emphasis on the linguistic reality of human experience (Clandinin and Connelly, 2000; Clandinin, 2007). As Webster and Mertova $(2007,4)$ comment, 'Narrative research does not claim to represent the 'exact' truth but rather aims for the 'verisimilitude' - that the results have appearance of truth and reality'. Thus, conclusions within narrative research generally remain open-ended (Webster and Mertova, 2007). The collection of a set of human experiences through the construction and reconstruction of personal stories is, as Webster and Mertova $(2007,1)$ promote, 'also well-suited to addressing issues of complexity and cultural and human centredness because of its capacity to record and retell those events that have been of most influence on us'.

Narrative inquiry is therefore seen as the most appropriate method for gathering data from key actors and middle-lifers as well as for understanding the complex socio-economic and cultural structures in a seaside resort. Of interest to narrative enquirers is not what happens so much as what meaning people attribute to what happens (Connelly and Clandinin, 2000). Connelly and Clandinin $(2000,2)$ note that 'humans are storytelling organisms who, individually and collectively, lead storied lives'. Thus, the study of narrative is the study of the ways humans experience the world. Understanding middle-life migration decisions and the influence of the 'psycho-social environment' (Florida 2008) requires an understanding of emotions and the complexity of characters, so narrative inquiry (Silverman, 2001; Webster and Mertova, 2007; Holstein and Gubrium, 2008) can be viewed as an appropriate methodology.

The primary objective, through a series of interviews, is to gather information on people's experiences and emotions, thus providing an insight into their motivations and perceptions. For middle-lifers this will relate to their reasons 
and motivations for migrating to a seaside resort, and their experiences both before and after the migration. The key agency interviews will seek to gather data on the interviewees' insights into the environment, the people and the image of the resort and their perceptions of the place, its governance and past, current and future regeneration. Having set out the epistemological reason for adopting qualitative research methods, in particular narrative inquiry and interviews, it is now argued that a single-case study is the most suitable approach to tackling the subject under investigation in its full complexity and revealing the contextual specificity demanded by the adoption of narrative inquiry.

\subsubsection{The Case Study approach}

Previous studies argue that a case study is an effective method for carrying out an extensive and comprehensive examination of a particular setting whilst also understanding the distinctive features of a case, or a set of cases, and associated complex social phenomena (Stake, 1995; Bryman, 2004; Hyde et al., 2012; Woodside, 2010). This approach enables this research to focus on both the narratives of recent middle-life in-migrants to a seaside town and those of the political and business actors seeking to address, or otherwise, the changing physical environment in the town. This approach engages with the social-political and cultural context that impacts on the regeneration landscape and the resulting governance practices that shape policy implementation at a local scale. Focussing on a single-case study acknowledges, in keeping with the Lamarckian perspective, that the key agencies and the middle-life migrants are part of the co-production of the physical environment, cultural norms, traditions, and practices which in turn shape the space they occupy and the social activities they implement.

There are two key justifications for the use of case studies in this research. The first justification is that the scope of a case study investigates a contemporary phenomenon, in depth and within a real-life context (Yin, 1981a, 1981b, 2009). Case study methods are used to understand real-life phenomena in detail, and such understanding encompasses important forms as 'contextual conditions, because they are rightly pertinent to the phenomenon of inquiry' (Yin, 2004, 13). 
People's life choices and perceptions are the focus of this research, and hence a case study provides the depth and insight necessary to understand these phenomena. Nonetheless, because phenomena and context are not always easily discernible in real-life situations, this provides a second justification for the use of case studies, 'in that the case study inquiry copes with the technically distinctive situation in which there will be many more variables of interest than data points' (Yin 2009, 18). This twofold justification illustrates that a case study methodology is suitable for understanding real-life situations, choices and perceptions (Patton, 2000; Yin, 2009; Woodside, 2010; Hyde et al., 2012). Furthermore, alongside contextuality, the current research aims to examine the influence of place-based factors such as the physical environment and people. As Agarwal (2002) noted from Cooke (1989, 40), 'it is impossible to understand universal processes without appreciating small scale local changes since localities are actively involved in their own transformation as they internally articulate to wider socio-economic processes'. Agarwal (2002) looked at both global and local factors conducive to coastal resort change in her studies of Torbay, and she recognised that it was not useful to look at national and international forces of social and economic change alone. Instead, thorough investigations focusing upon small-scale, sub-national localities were also necessary, in order to evaluate specific changes on the ground. Accordingly, a range of arguments is developed below favouring one case study type which is suitable for understanding local factors and spatial specificity whilst being in keeping with the theoretical, epistemological and methodological approaches of this thesis outlined earlier in the chapter.

Case study designs can either be single or multiple. Single-case studies use either a holistic design or embedded units of analysis (Stake, 2000; Yin, 2003a, 2003b, 2009). Overall, the single-case study design is eminently justifiable under certain conditions (Yin, 2003b) - where the case represents either/or a critical test of theory, a rare or unique circumstance, a representative or typical case, is revelatory, or is longitudinal in purpose. Within the chosen case study it is considered that the categories applicable fit the rare or unique group, though it may also be revelatory, depending on the outcomes of the research. Later in this chapter, greater detail is provided as to why Bexhill-on-Sea is 'rare' because of its distinctive and high level of in-migration of an elderly population 
and seaside deprivation. Within the single-case study there may be incorporated sub-units of analysis, so that a more complex embedded design is developed (Yin 2003b). This is not a case study within a case study; rather, the sub-units may offer opportunities for further insights into the single-case study. However, care must be taken when analysing these sub-units, as the larger holistic aspects of the case may be ignored, and so the case study itself may change and shift its orientation by moving away from the research aims and into a more specific line of inquiry (Yin, 2009). The sub-units in relation to the Bexhill-on-Sea case study relate to the Lamarckian perspective and the analysis of socio-economic changes in the town linked to in-migration. These sub-units of analysis are the physical environment, key agencies' role in the regeneration of seaside towns (and their governance and leadership), middlelifers' migration motivations and behaviours and images and perceptions of the town. These sub-units were the focus of this research, and so from this theoretical standpoint their interconnections can be used to develop a fuller analysis of the case study as a whole. The case study therefore allowed the analysis and interpretation of the key agencies of change, and how they perceive their attempts to influence the physical environment, and the effects of these changes. It also enabled a better understanding of in-migration motivations of middle-lifers seeking resort retirement.

Both Yin (2009) and Bryman (2004) consider the limitations of the single-case study strategy when aiming at generalising findings beyond the immediate case study. Yin (2003a, 61) claimed that 'most criticisms about single-case studies reflect fears about uniqueness or artifactual condition surrounding the case,' which may eventually result in uncertainty concerning the quality of the empirical research being conducted. Promoters of the multiple-case study strategy claim that this form is more likely to be stronger than the single-case study, as it is believed to produce a stronger effect because the evidence from multiple-case studies is often considered more compelling (Clandinin, 2007; Woodside, 2010; Hyde et al., 2012). Despite emphasis on the multiple-case study strategy, however, there are strong arguments for a single-case study approach, especially given the researcher's aims and objectives and the theoretical perspectives of this thesis. 
Stake (2006) proposed a narrative of the 'contextuality' of each 'particular' individual case study, as each has important features, happenings, relationships and situations. Stake (2006) argues that researching the 'contextuality' of a 'particular' case actually competes with the search for 'generalisability' pursued by some social scientists. The argument for drawing 'generalisations' from case study findings is neither the goal of every case of qualitative research, nor should it be emphasised in all research (Stake, 2006). Hence, multiple-case studies, it has been argued, can be one of the epistemological strategies that obscure case study knowledge and paradoxically fail to 'facilitate comparison' (Stake, 2006; Woodside, 2010; Hyde et al., 2012). It has been argued, therefore, that the focus of the case study should be the specific case itself, as a comparison actually competes with learning from the particular case - and so uniqueness and complexities are glossed over (Flick 2006). In this respect, Flick (2006) advocated that in a multiple-case study it is not necessary to observe the case as a whole but rather only a particular excerpt. Accordingly, a single embedded case study design aligns better with the aim and objectives of this research than multiple-case studies manage to do.

The literature review identified that endogenous factors, such as the behaviour of local governance, political decision-making, planning influences and leadership in the business or political community, can play a significant part that can set the tone or direction of a resort (Agarwal, 1995, 1997, 1999a, 1999b; Shaw and Williams, 1997; Russell and Faulkner, 2004; Agarwal \& Brunt, 2006; Shaw and Coles, 2007; Margate Renewal Partnership, 2008). These in themselves can be as influential, if not more so, than past and concurrent exogenous forces and are seen as the main challenge for regeneration practitioners. There are those who believe that understanding these complex characteristics of endogenous forces will in itself help unlock seaside resorts' future potential (English Heritage and Urban Practitioners, 2007; Future Policy Thinking, 2009), while Marsden $(2003,54)$ argues that 'one size fits all is a motto you apply at your peril to seaside resorts'. The single-case study is therefore a suitable approach to take, since its depth will allow an understanding of the complex forces that have changed seaside towns whilst also achieving the depth of understanding that Agarwal (1996) argues is 
required to analyse the local specificity of processes of change in coastal locations.

Besides the theoretical and methodological justifications supporting the choice of a single-case study strategy, 'convenience' and 'accessibility' criteria need to be acknowledged as well. Silverman $(2005,129)$ claimed that very often a case is chosen simply because 'convenience or accessibility guides selection' which will allow access, and so thereafter feasibility is the basis of the decision to undertake a single-case study. Bryman (2004) and Flick (2006) discussed 'accessibility' as a crucial factor, due to the need to become closely involved in the field and so generate human conditions to create enough trust for the participants, especially key informants, to disclose their views on the subject under consideration. This may lead to a snowballing approach (Bryman, 2004), where the researcher makes initial contact with people who are relevant to the research subject and then uses these people to establish contacts with others. These key informants are often critical to the success of a case study (Bryman, 2004; Silverman, 2005; Flick, 2006). Access to key informants in Bexhill-onSea was a particular strength the researcher could bring to this case study and is discussed later in the chapter. Convenience and accessibility, therefore, were further rationales for the single-case study, along with theoretical and methodological justifications. The next section further justifies the choice of using a single-case study, by providing empirical detail on the 'rare' social and economic characteristics of Bexhill-on-Sea.

\subsubsection{Bexhill-on-Sea as a single-case study}

Given the existing social structure of Bexhill-on-Sea, as presented in earlier chapters but further expanded on in the next chapter, it was considered a suitable case study, in that it is a resort that has undergone socio-economic changes that have been influenced by key actors and the migration of middlelifers and the retired. It therefore exemplifies the social characteristics that this thesis wishes to examine, as it has a particularly high concentration of elderly and middle-lifer residents (see next chapter). Furthermore, as also discussed in Chapter 5, a complex set of forces has historically influenced the physical environment and has had profound implications on the town's demographics. In 
addition, recently an increased emphasis on regeneration amongst key agencies has also affected the physical environment of the town. The combination of changes to the socio-economic structure, linked to the 'retirement' migration of middle-lifers and changes to the physical environment makes Bexhill-on-Sea, on the Sussex seaside coast, a useful case study in line with the theoretical aims of the research.

Overall, Bexhill-on-Sea currently has the distinct social and demographic characteristics that make it a suitably 'rare' single-case study. A case study approach and the related methods of data collection are not independent of one another; they are indeed mutually supportive and related. The research design outlined in the first part of this chapter therefore needs to be viewed as the framework for the specific collection and analysis of data in the case study outlined below in part two of the chapter (Huberman and Miles 1994; Yin 2009). The sections that follow in part two not only consider the detail of the methods adopted in the case study but also discuss issues such as validity, authenticity and credibility. The next section therefore provides details regarding the research design and methods used for the case study of Bexhill-on-Sea. Continuing to draw on an epistemological approach that stresses the situated nature of knowledge, the single-case study approach is usefully adopted here, in order not to identify the truth but rather to offer interpretations of realities and to situate critically those interpretations in the local context (Hyde et al., 2012; Woodside, 2010). The research aims to involve the understanding of 'lived experience' (Yin, 2009; Silverman, 2010) of middle-lifers and key informants, and this is explored through open-ended interviews which are discussed in more detail below.

\section{2 (Part 2) - Research Design}

The previous section explained the narrative inquiry methodological framework and the use of interviews and documentary evidence as the most appropriate methods of gathering data. This section now provides details on how these methods were utilised and the key challenges encountered and addressed in this respect. 
Firstly, the research design included semi-structured, in-depth interviews with key actors from key agencies (statutory and non-statutory) (Table 4.1) and with middle-lifers who had moved to Bexhill-on-Sea in the past five years (Table 4.2).

\begin{tabular}{|c|c|c|}
\hline \multicolumn{3}{|l|}{ Key Agencies Interviewed } \\
\hline $\begin{array}{l}\text { Statutory Agencies } \\
\text { (number code starts with 2) }\end{array}$ & Who & Date \\
\hline Rother District Council & Senior Member of the Council & Oct 2011 \\
\hline Rother District Council & Senior Officer of the Council & June 2011 \\
\hline Rother District Council & Planning strategist & July 2011 \\
\hline Rother District Council & Regeneration practitioner & June 2011 \\
\hline East Sussex County Council & Regeneration practitioner & July 2011 \\
\hline $\begin{array}{l}\text { Hastings and Bexhill Regeneration } \\
\text { Company (SeaSpace) }\end{array}$ & Senior Officer & July 2011 \\
\hline \multicolumn{3}{|l|}{$\begin{array}{l}\text { Non-statutory Agencies } \\
\text { Interviewed } \\
\text { (number code starts with 3) }\end{array}$} \\
\hline Sainsbury's & Regional Store Manager & Nov 2011 \\
\hline $\begin{array}{l}\text { Land Securities } \\
\text { (Ravenside Retail Park) }\end{array}$ & Regional Property Advisor & Nov 2011 \\
\hline $\begin{array}{l}\text { Hastings Direct } \\
\text { (Insurance Broker) }\end{array}$ & Director & July 2011 \\
\hline $\begin{array}{l}\text { Furness Controls } \\
\text { (High tech manufacturing) }\end{array}$ & Senior Director & Sept 2011 \\
\hline De La Warr Pavilion Charitable Trust Ltd & Senior Officer & July 2011 \\
\hline $\begin{array}{l}\text { GabyHardwicke } \\
\text { (Solicitors) }\end{array}$ & $\begin{array}{l}\text { Senior Partner } \\
\text { (past President of Chamber of } \\
\text { Commerce) }\end{array}$ & Oct 2011 \\
\hline Peterhouse retirement home & Senior Staff Member & Nov 2011 \\
\hline $\begin{array}{l}\text { Independent Retailer } \\
\text { (Picture frame shop) }\end{array}$ & Owner & Sept 2011 \\
\hline $\begin{array}{l}\text { Independent Retailer } \\
\text { (Newsagent) }\end{array}$ & Owner & Sept 2011 \\
\hline $\begin{array}{l}\text { Independent Retailer } \\
\text { (Antiques shop) }\end{array}$ & Owner & Oct 2011 \\
\hline
\end{tabular}

Table: 4.1 List of principal key agencies researched and key actors interviewed

The purpose of the interviews with key actors was to understand the context in which they currently operate, how these actors consider their role in the regeneration process and how they might impact upon this in the context of a declining socio-economic base and an ageing population. 16 semi-structured interviews were conducted with key actors from local agencies. Six of these 
were with representatives from statutory agencies (these were the local authorities and a government grant-funded regeneration company), commencing with the interview code number (2), and ten were with representatives from non-statutory agencies (these being businesses and third sector organisations), commencing with the interview code number (3) (Table 4.1). This selection of non-statutory and statutory sector interviewees ensures that the key actors interviewed included a mix of those who were formally involved in the planning and regeneration process through their job and those who were informally involved, usually through their role in a non-statutory body. A further reason for choosing both non-statutory and statutory agencies was to gain an understanding of the town, its residents and image from different perspectives of governance and business.

The interviews with recent migrant middle-lifers (Table 4.2) sought to understand their motivations for migration to a seaside town and whether the physical environment, the people, the town's image and the 'personality' of the place, as discussed in previous chapters, had had an influence on their motivations.

\begin{tabular}{|l|l|l|c|}
\hline \multicolumn{4}{|l|}{ Middle-life Migrant Interviews } \\
\hline $\begin{array}{l}\text { Middle-life migrants to Bexhill- } \\
\text { on-Sea age categories } \\
\text { (number code starts with 1) }\end{array}$ & $\begin{array}{l}\text { 21 semi-structured } \\
\text { interview codes }\end{array}$ & $\begin{array}{l}\text { Invitation via } \\
\text { District-wide } \\
\text { magazine and RDC } \\
\text { citizen panel }\end{array}$ & $\begin{array}{l}\text { Date of } \\
\text { interviews } \\
\text { Sept-Oct } \\
2011\end{array}$ \\
\hline $50-55$ years of age & Coded (a) & $\checkmark$ & $\checkmark$ \\
\hline $56-60$ years of age & Coded (b) & $\checkmark$ & $\checkmark$ \\
\hline $61-65$ years of age & Coded (c) & $\checkmark$ & $\checkmark$ \\
\hline $66-70$ years of age & Coded (d) & $\checkmark$ & $\checkmark$ \\
\hline
\end{tabular}

Table: 4.2 List of middle-life migrants to Bexhill-on-Sea in the past five years

There were 21 semi-structured interviews with middle-lifers, commencing with the interview code number (1) (Table 4.2), who had migrated to Bexhill-on-Sea in the past five years, with an even spread of ages between 50 and 70 years of age (ending in letters 'a' 50-55; 'b' 56-60; 'c' 61-65; 'd' 66-70). Appendix 11 provides further background information on these middle-life participants. The choice of methods for data gathering through semi-structured interviews reflects 
the qualitative methodology set out in the previous sections. The approach to undertaking the semi-structured interviews is discussed below, but more generally they maintained the exploratory attitude needed to uncover aspects of Bexhill-on-Sea's regeneration omitted from available official literature, such as the complex relationship between image, the physical environment and people's choices.

The second aspect of the research design is documentary analysis of secondary sources (Table 4.3), organised accordingly to illustrate meaning. This focused on understanding the nature of deprivation within the town, governance issues and decision-making, which required reading through previous committee reports and minutes, reviewing strategic documentation from planning and regeneration departments, evaluating documentation from the non-constitutional Bexhill Town Forum and reading past and current local newspapers.

\begin{tabular}{|c|c|c|c|}
\hline \multicolumn{4}{|l|}{ Secondary Data Analysis } \\
\hline Analysis & Sources & $\begin{array}{l}\text { Research } \\
\text { approach }\end{array}$ & Date \\
\hline $\begin{array}{l}\text { Indices of multiple deprivation, } \\
\text { neighbourhood demographics } \\
\text { and employment data }\end{array}$ & $\begin{array}{l}\text { East Sussex in Figures } \\
\text { (ESiF) Office of National } \\
\text { Statistics (ONS) }\end{array}$ & Desktop & $\begin{array}{l}\text { July } 2011 \text { - } \\
\text { March } 2012\end{array}$ \\
\hline $\begin{array}{l}\text { Cabinet, scrutiny and working } \\
\text { party reports and minutes }\end{array}$ & Rother District Council & Desktop & $\begin{array}{l}\text { January } 2000 \\
\text { - June } 2012\end{array}$ \\
\hline $\begin{array}{l}\text { Corporate strategies, } \\
\text { regeneration plans and planning } \\
\text { approvals relevant to Bexhill-on- } \\
\text { Sea }\end{array}$ & Rother District Council & Desktop & $\begin{array}{l}\text { January } 2000 \\
\text { - June } 2012\end{array}$ \\
\hline $\begin{array}{l}\text { Bexhill Town Forum agendas and } \\
\text { minutes }\end{array}$ & Bexhill Town Forum & Desktop & $\begin{array}{l}\text { January } 2006 \\
\text { - June } 2012\end{array}$ \\
\hline $\begin{array}{l}\text { Hastings and Bexhill Taskforce } \\
\text { and SeaSpace agendas and } \\
\text { minutes }\end{array}$ & SeaSpace & Desktop & $\begin{array}{l}\text { January } 2003 \\
\text { - Sept } 2011\end{array}$ \\
\hline The Bexhill Observer & $\begin{array}{l}\text { The Observer group of } \\
\text { newspapers }\end{array}$ & Desktop & $2004-2013$ \\
\hline
\end{tabular}

Table: 4.3 Secondary analyses of documentary sources

Justifications for the selection of these sources, and the approach taken to their analysis, are discussed in the sections below. 


\subsubsection{Sampling}

As stated earlier in the chapter, aside from a theoretical and methodological perspective, the 'convenience' and 'accessibility' justifications supporting the choice of single-case study strategy were equally relevant. The position of the researcher as Executive Director at Rother District Council gave the 'convenience' and the opportunity to overcome 'accessibility' constraints. This enabled the researcher to follow the arguments of Flick (2006) and to become closely involved in the field, as this created human conditions that generated 'enough trust' for the participants to disclose their views on the subject under investigation. In the case of the key actors who were interviewed, some were known to the researcher in their professional capacity. However, with the middle-lifers, none was known to the researcher, and so this involved negotiating the role and position, as the researcher had to gain trust and confidence as a professional stranger (Agar, 1986).

The key statutory agencies, their officers and elected officials were all known to the researcher. Purposive sampling was adopted in this instance, in that the organisation was selected based on the researcher's knowledge of their strategic corporate aims and objectives and those departments that would best respond to the purpose of the study. Through personal knowledge and documentary sources, a direct approach was made to the District and County Council's Chief Executive Officer and leader and their regeneration and planning departments, seeking guidance on the most suitable interviewee for the purposes of this research. Also, a direct approach was made to the Chief Executive of the local arms-length regeneration company SeaSpace (now Sea Change), and in all cases it was the more senior officials and elected members that chose to participate in the research interviews. The non-statutory agencies (Table 4.1) were sampled through a mixture of purposive sampling through personal knowledge and documentary sources. In the instance of the De La Warr Pavilion, this was selected because it was cited within the District Council's Corporate Plan and Regeneration Strategy as being central to the regeneration agenda for Bexhill-on-Sea. Hastings Direct and Sainsbury's were purposely selected as the main employers within the town, while Land Securities had been largest investor in the town over the past four decades. 
These organisations were contacted directly by the researcher, and senior local officers agreed to be interviewed. With the other non-statutory agencies, such as retailers and SMEs, these were approached through networking forums such as the Chamber of Commerce, street traders' associations and documentary sources working closely with the local authority's regeneration department. There was only one instance of snowballing (Flick, 2000; Silverman, 2005) in the retailing sector.

Apart from two, all the interviews were conducted on the respondents' work premises at an agreed time and at their convenience. The two agencies that were unable to accommodate the interviews were offered an alternative venue by the researcher that was acceptable and convenient to them. Direct approaches by telephone were followed up with an e-mail confirming the date and the time of the meetings. The e-mail incorporated attachments, including an information sheet on the researcher, what the research was about and details regarding confidentiality. It also included a participant consent form, which would need to be signed before the interviews commenced (Appendices $12 a$ and $12 b)$.

With regards to the middle-lifers that were interviewed, these were approached using Rother Council's citizen panel, with qualifying criteria that they had moved to Bexhill-on-Sea within the past five years and were within the ages of 50-70. This had full ethical consent from Rother District Council's statutory 'Monitoring Officer'. The citizen panel is a database of residents across Rother who have registered to participate in surveys and interviews and which has over 1,800 registrations. In addition, the researcher had the Council's permission to include an approved recruitment advert in its quarterly newsletter that also goes out to every household in Rother. The quarterly newsletter incorporated a feature on regeneration in Bexhill-on-Sea, whereby residents were invited to take part in further research on the subject. In replying they were asked to identify age range, gender, problems that may restrict their participation, the area in which they lived and how long they had lived in the town. The responses to these two approaches provided the researcher with over 233 interested participants. The researcher then worked with the Council's database administrator to identify 20 middle-life willing participants in five-year 
age bands with a mix of genders, disabilities, postcode variables and with five potential respondents kept in reserve in case of participants dropping out. Taking account of respondents' geographical postcodes and any disabilities was considered important, as the literature review identified marked variations in the characteristics of neighbourhoods within seaside towns, such as HMOs in the past tourism accommodation stock, and also higher than average disability claimants in seaside towns (Agarwal, 2002, 2007; Beatty et al., 2003, 2008; Margate Renewal Partnership, 2008). The 21 middle-life interviews, along with the 16 statutory and non-statutory interviews, were sufficient for the purposes of gathering adequate data on the case study location and addressing the research question, as suggested by writers on the case study approach and narrative inquiry, who all recommend keeping research sampling to a manageable size (Crotty, 2003; Clandinin, 2007, Yin 2009, Silverman, 2010). In the event, none of those 20 middle-lifers approached withdrew, but the researcher also used one of the pilot middle-life interviews, as this was conducted using the final draft of the interview schedule and held useful data. In total, 37 one-hour interviews were conducted that delivered a wealth of data that provided a sample size that was more than sufficient. This was a manageable size, as from the time of the first interview, transcribing the tapes, accounting for field notes, data analysis and theme generation took nine months to action.

\subsubsection{Interviews and questions}

Bryman (2004) argues that interviews are probably the most widely employed method in qualitative research. However, key determinants of the choice of the most suitable interview type concerned the negotiation between 'openness' and 'focus'. 'Openness' was preferred to 'focus', although 'focus' was needed for obtaining data on certain key social interviewee characteristics, such as age and gender. The choice of 'openness' as an approach to the semi-structured interviews for the middle-lifers was to maintain the exploratory attitude needed to uncover their migration motivations while at the same time triggering followup and probing questions that maintained a focus that avoided the interview being taken away from the researcher's aim and objectives (Bryman, 1988, 2004; Mason, 2002; Silverman, 2005). 
The more flexible semi-structured interview method described also changes the balance of researcher/interviewee control over the direction of discussion, and so it facilitates a more open type of questioning that goes some way to redressing the power imbalance of a structured interview while still addressing topic(s) of interest to the researcher (Brewer, 2000; Mason, 2002). This open, relaxed and more equitable research scenario was desirable from the epistemological position of the researcher discussed above, as this open structure offers participants a greater say in the interview and the direction of the research. It also offers the researcher an opportunity to better address complex social processes and helps moderate distortions to replies caused by the 'interviewer effect' (Brewer, 2000; Mason, 2002; Bryman, 2004, Silverman, 2006,2010 ) in an effort to elicit an honest and natural voicing of opinions and representations by the participant. An identical approach was adopted for the key agency actors, as this openness gave the power to the 'professional' officer or politician to inform the researcher, in a semi-structured controlled interview method, of their understanding and perceptions of the seaside resort's complexities.

The questions were influenced and informed by the research aims, the theoretical context and previous research. They were therefore devised under certain headings, but in such a way as to allow for 'openness' while maintaining 'focus'. The key actor questions explored their perceptions of Bexhill-on-Sea's past and present socio-economic performance, as well as the cultural and built environment. Their views on the influences and roles that had shaped the town in the context of the environment, the people and its image were also explored. The interview questions also sought to investigate their future views on how regeneration may be taken forward and what required adapting in terms of the physical environment, demographics and the image of the town (Appendices $13 a, 13 b$ and 13c). By conducting interviews in their chosen environment, the researcher positioned themselves as a research student. Moreover, facilitating an introductory chat about them and their organisation, before the interview commenced, gave them a feeling of being in control and the chance to put forward their views and perceptions in an open and transparent manner. 
The structure of the interview questions for the middle-lifer interviews was slightly different to that of the key agencies, to take account of their migration motivations along with their perceptions of the physical environment, the residents of Bexhill-on-Sea and its image, both before and after they migrated. The interviews were piloted on four participants (two middle-lifers and two statutory agencies), to inform adjustments to the final interview questions. Three of these interviews were discarded and treated as confidential, but one middle-lifer interview was retained, as the data gathered were the same as in the final interview question structure. Such adjustments to the questions included clarification on the environment question pertaining to the urban and natural settings, adjustment to the sequencing of the questions and, in the case of the middle-lifers, simplification of the language used. The early questions in the final question schedule allowed participants to introduce themselves and describe their personal history, which in turn helped them to relax and engage with the researcher. This then flowed into questions investigating their reasons for migrating to Bexhill-on-Sea. The interview then explored their perceptions of the place before they arrived, and why they had chosen this particular town. It also examined whether they felt their perceptions lived up to their 'experience' and their views on the environment, the socio-economic structure and the personality of the place once they had lived in the town for a while. The questions also explored how they perceived the future of the town and the role of statutory, non-statutory agencies and residents have in regeneration and what (if anything) needs adapting. The final part of the interview was not recorded but captured some background information on paper concerning how many people made up the household, their relationships, the property type, ownership and household income. The interview guides for middle-lifers are provided in (Appendix 14).

Recorded interviews that would be viewed as text from which to draw 'units of data' (Saunders et al., 2000), and that could be used to conduct an accurately contextualised analysis later on, after the end of the data collection stage, was the approach adopted in all interviews. Every participant was agreeable to being recorded, which enabled the interviewer not to have to make copious notes but rather concentrate on the interview and more effectively utilise the semi-structured interview to elicit data. 


\subsubsection{Analysis and coding}

This section details the process of data analysis and coding and provides a justification of this element of the research design in satisfying the research aims. To ensure robustness of the research, the data were analysed using techniques and practical procedures such as constant comparative analysis, reflective notes, respondent validation, corroboration of sources and methods and a clear audit trail, and it also used analysis processes involving 'coding' and 'indexing', as described by Strauss and Corbin (2008).

Analysis techniques adopted an inductive thematic approach (Brewer, 2000) that was in keeping with the overall narrative inquiry, in order to view the interview texts as 'stories'. Thematic analysis was used to identify themes coming through the data about the environment, people, the town's image and its governance. Once these were established, relationships between the themes were identified with reference to the research focus on the socioeconomic and cultural structure of the seaside town and the in-migration of middle-life people. Thematic analysis was employed, by drawing on a process outlined by Mason (2002) and Strauss and Corbin (2008). This was combined with elements of discourse analysis, to give a fuller exploration of the nature of perceptions and stories identified during thematic analysis. This analytical approach was consistent with the richness and depth of data captured and was used to address the research questions.

The analytical process for the interviews, and how it links to the documentary analysis, is detailed in (Figure 4.1.). The analysis process first involved a coding and indexing exercise. This is described also as a mechanism for thinking about the meaning of data collected (Huberman and Miles, 1994), when the emerging categories, according to Dey (1993), must be meaningful in relation to the data and 'to the other categories' as well. Accordingly, Strauss and Corbin $(1998,2008)$ suggested that the names and labels to be employed for this coding and categorisation exercise can draw on terms that emerge from the data as well as terms used in existing theory and literature 


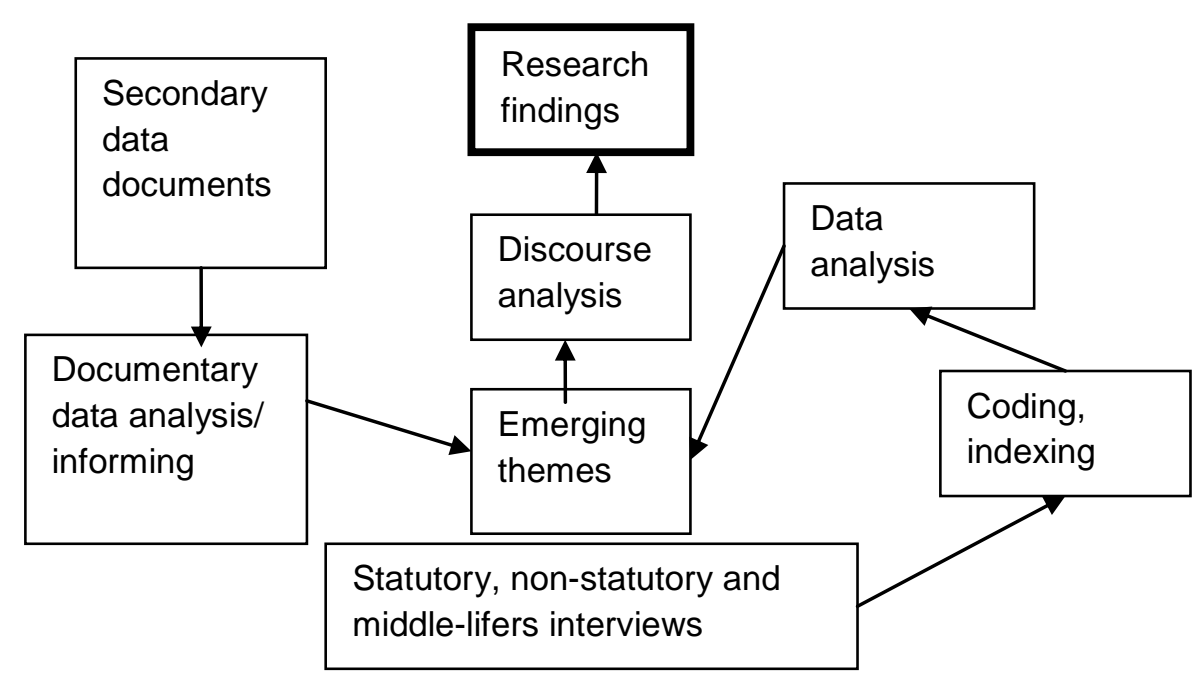

Figure: 4.1 Analytical processes

'Source: Adapted from Strauss and Corbin (2008)'

Thematic analysis is not a linear process, and so many steps were repeated or fed back into earlier steps over the course of the analysis, which is in keeping with in-depth narrative inquiry. This is particularly true of the coding steps that often involved returning to the data and redefining, subdividing, removing, merging or creating new codes. In the most part, analysis was done once each set of interviews was completed, though there was of course initial interpretative analysis during transcription after each meeting, reflections on field notes and text reading. This iterative approach allowed the researcher to check back against older data as interpretations developed, as well as re-directing the data capture process accordingly. The nature of coding involved identifying codes as they emerge from the data, but, in keeping with the epistemology and methodology outlined earlier, the coding responded to the research aims and the existing literature detailed in previous chapters.

The coding and indexing process was conducted using Computer Assisted Qualitative Data Analysis Software (CAQDAS), in this case NVivo9 software, to assist in managing the lengthy, complex and discursive profile of the data. Using constant comparative analysis, reflective notes and analytical processes, four parent headings were identified: Environment, People, Image and Governance. Under these parent themes, 50 nodes were identified, which are reflected in Chapters 6 and 7 that seek to offer this analysis in a systematic and presentable way. When demonstrating these themes and patterns in the 
analysis chapters, qualitative descriptions are provided with supporting evidence, in the form of data quotations from interviews, and references back to field note extracts, with the intent of providing 'thick descriptions' that improve the reliability of the narrative inquiry and findings. The steps that were followed during the coding process included, firstly, familiarisation with the texts, i.e. reading and re-reading, and secondly, open coding or literal reading. This involves looking at the data for recurring and outlying literal meanings, structures and forms (i.e. indexing the data into categories) and does not at this stage include interpretation. Thirdly were interpretative indexing categories, with the identification of broad groups of categories drawn from within the open codes through the initial interpretation of themes. This is dominated by a process of synthesis and a review of multiple open codes, and it places interpretation within the context of the research question (Mason, 2002)

By collating the open codes into the four parent themes, more nuanced indexing was possible when recoding for interpretative codes. For instance, under the Environment theme there were originally 27 indexing codes, such as 'tiredness' and 'blockages to regeneration', with 336 individual sources. Under the Image theme there were originally 17 indexing codes, such as 'granny-ville' and 'quaint, genteel, old fashioned', with a 185 individual sources supporting the Image theme. This enabled the process of analysis to advance beyond the literal observation to a narrative and interpretive level that situated the findings within the broader academic debates. The data analysis software facilitated the identification of connections, overlaps and exceptions within the codes already identified (Mason, 2002). These codes were then re-situated (elevated, demoted, recoded) within the new interpretative code structures.

The next subsection provides a discussion of the procedures employed to help secure the validity, authenticity and credibility of the research design, methods and findings, as discussed earlier in the chapter.

\subsubsection{Validity, authenticity and credibility of the research findings}

There are a number of different validity, authenticity and credibility criteria the academic community uses to evaluate the quality of empirical qualitative 
research in terms of research design, practice and conclusions (Flowerdew and Martin, 2005). Mason (2002) suggests that demonstrating this can be achieved by showing that the research design provides appropriate tools for addressing the research question and specifically generating the data needed to answer this question. Mason (2002) also proposes that for a test of authenticity and credibility, the researcher must be able to demonstrate the methodological, theoretical, ethical and practical steps taken, in order to convince the reader of the careful design, the appropriateness of methods and accuracy of data collection.

The research design has evolved from the author's emotionalist epistemology, which in turn is compatible with a narrative inquiry methodology and the qualitative methods of interviews and documentary analysis. Analytical procedures were employed to understand the meaning of the validity and authenticity of research design and findings, which included reflective practice (May 2001, Hay, 2005), respondent validation (Yin, 2009), a clear audit trail (Brewer, 2000), systemisation of the process (Strauss and Corbin, 2008), a dissemination strategy (Hay, 2005) and adherence to formal universitysanctioned ethics procedures.

Steps taken to improve the validity and authenticity of findings were reinforced by efforts to achieve 'subjective adequacy' and systemisation of the process, by utilising the six specific indices designed by May (2001). First, a prolonged period of time was spent in the town, in order to gain a greater understanding of its culture. The research involved six months in the field and three months advance scoping, although the researcher had been working in the environment for the past 20 years. Second, concentration on the physical setting of Bexhillon-Sea was designed to improve consistency, relevance and understanding. Third, diverse and multiple people, with different experiences of the local culture, were included in the research, which, according to May (2001), can improve depth of understanding and findings. Points of interaction with the participants included attending the same meetings as well as the interview over a long period of time, which did improve insight into the multifaceted nature of representations expressed in the interviews. Fourth, the language used by the key agencies was already familiar to the researcher as Executive Director at 
Rother District Council, and this familiarity consequently reduced barriers to understanding between both parties during the interviews. Fifth, developing relationships with participants, both the key agencies and the middle-lifers improved the researcher's understanding of meaning - inviting the middle-life participants to introduce themselves and describe their personal history smoothed the process in this relationship. Sixth, the methods were designed to promote a shared understanding between the researcher and the participants, in order to improve the validity and authenticity of the interpretations. By following an honest and comprehensive ethical procedure (including respondent validation, clearly informed consent and research dissemination to the participants), the researcher sought to break down any misunderstandings and ensure clarity for all parties involved.

\subsubsection{Reflexivity, bias and ethics}

The interview transcripts were stored electronically on a password-protected computer and also on an encrypted USB stick. Hard copies are stored in a secure location, with researcher access only. Ethical checks were presented to the University of Brighton's School of Environment and Technology's ethics committee and discussed with supervisors. The researcher also agreed with Rother District Council's statutory 'Monitoring Officer' in regard to the researcher's approach to securing data, its confidentiality, respondent validation, informed consent and the coding of interview data, to ensure anonymity. The transcripts attributed to the participants were only viewed by the researcher and university supervisors.

There are two reasons for including reflective practice in this research design. Firstly, in terms of the research findings, it is necessary to position the researcher's knowledge-making practice within the personal, institutional and cultural contexts in which the research is conducted (Ibarra and Kusenbach, 2001; May 2001), as this contributes to the increased validity of the work. Secondly, by developing a research skill that means reviewing the projects from a reflexive standpoint, this hopefully allowed the researcher to identify an opening for the research in areas and perspectives previously not considered (Mason, 2002). Reflective practice and diary notes were employed, in an 
attempt to make explicit any bias or ethical issues that arose from the researcher's relationship with other key agencies (Ibarra and Kusenbach, 2001; McGill et al., 2002; Jasper, 2003).

Those key agency individuals known to the researcher were all interviewed on their own premises and were receptive to a recorded interview. At the commencement of each interview the researcher emphasised the points provided in the 'meetings information sheet' (Appendix 12b) and their role as a researcher and not as an employee of Rother District Council, as well as the importance of confidentiality. Field notes were kept, before, during and after the interviews, and these were placed with the transcripts to enable the researcher to reflect on issues of bias. However, the researcher's position in Rother District Council did allow immersion in the subject area and was seen as useful for achieving authenticity (May 2001 and Jacobs 1993). The research design therefore addressed this potential bias through respondent validation, by getting them to agree to and sign the meeting information sheet and confidentiality form and at the end of each interview, thereby ensuring the participant understood what actions to follow if they believed I had misled or mismanaged the interview, and how to complain. Also, the need for a clear audit trail where the research material (interview tapes, transcripts, field notes) and the analysis using CAQDAS (NVivo9) and sign-off with the participants is all auditable, either on the computer-protected software or in file notes held in a secure location, was addressed. On no occasion since the interviews have any complaints been received or concerns raised with the researcher, the university or the researcher's sponsor. Rother District Council is the sponsor of the research, on the proviso that the final thesis is submitted to the Council for consideration alongside their regeneration strategy and corporate plan. There has been no influence by the sponsor on the outcome of the thesis, though the executive position held by the researcher has ensured that the outcomes of the findings are keenly anticipated.

The key ethical issue for the researcher concerned the statutory and nonstatutory interviews, where the researcher was known to the participant and with whom the researcher was familiar through their position at the local authority. This issue was addressed in early verbal and written communications 
by being honest and explicit about the sponsorship and also the independence of the work in any communication or contact. The 'meetings information sheet' was discussed and the 'consent form' signed ahead of any interview commencing. The interviewer also ensured the supply of high-quality documentation and communication for all interviewees concerning the nature of the project.

The researcher did not identify any ethical issues that arose from using the citizen panel or the Council newsletter. As stated earlier, the use of the panel to recruit respondents was approved by Rother District Council's statutory 'Monitoring Officer'. More importantly for the researcher were the ethical issues surrounding the interviews with the middle-lifer's. All interviews were held in the participants' own home, to ensure safety for both the researcher and the participants. The researcher also adopted the Council's own 'Dignity at Work Act guide', 'Equalities Scheme' and the 'Safe Working Practices Guide for Travelling Officers'. Prior to the interview the participants were sent the consent form and information sheet (Appendices 12a and 12b), and upon arrival at their home and before entry, the researcher introduced himself and produced his student identity card. The researcher took into consideration aspects such as dress code and care within the home, such as laying a soft cloth on their table on which to support the recording machine, thus making the participant feel valued. The researcher would then start by introducing himself, as set out in the 'meetings information sheet', explaining the interview procedure and getting the form signed prior to commencement. All interviews ran smoothly, although at the end of one interview a participant suddenly felt unwell and the researcher had to call a neighbour who was an occasional carer to give them some support. Within two days after completing each interview, all participants received a letter/e-mail of gratitude from the researcher.

The researcher also followed all procedures as per submission to the university's ethical committee (Appendix 15). The University of Brighton's School of Environment and Technology's ethics committee application and approval also informed the research design and was a formal process designed to ensure the quality of the research project and its findings. The ethics application discussed measures integrated into the research design, to ensure 
the highest possible quality of the research process and data collection. These measures included: respondent validation, informed consent, project information sheets, reflective practice, research dissemination to participants and coding data to achieve anonymity. The scoping and piloting aspects of the project were also approved by the ethics committee, and the research did not commence until full ethics committee ethical approval had been given.

\subsubsection{Self-awareness and reflexivity}

Self-awareness and reflexivity issues in qualitative research are bound up with the research process as a whole and, in turn, with the aspect of research evaluation. This debate stems from the assertion that no matter how neutral, objective and detached the qualitative researcher is during the process of data gathering and analysis, the product of any research is a representation of the reality that is under scrutiny (Flick, 2007). According to Janesick (1998), qualitative researchers accept that there is no 'value-free' or 'bias-free' research design. As such, the qualitative researcher has to identify in the early stages his or her biases and articulate the ideology or conceptual framework for the study. Central to this argument is the idea that the researcher's identity is the main driver of research and that the research literature conveys the importance of understanding how research is shaped by the actions and the values of the researcher and the many negotiations within this context. Qualitative research requires an engagement in which the researcher's values and subjectivity are part of the construction of knowledge (Dwyer and Limb, 2001). The key assertions from this notion, in postmodern and post-structural research of neutrality, are unattainable 'because the researcher and the research cannot be meaningfully separated and that the researcher acts on the world and the world acts on the researcher, on a loop' (Wetherell et al., 2001, 54). These issues are important, as they can determine the quality of the research outcomes, the rigour of the research process and the objectivity of the findings.

These issues were discussed with the supervisors and the ethical approval panel, and as this chapter has shown, they were carefully managed and monitored throughout the research process and interpretations. The researcher, for instance, had previous training in equality issues, works in a 
highly confidential environment with sensitive data, has had training on database and records management, attended social, media and presentational methods and therefore had a sound grasp of how to adapt to and work with different audiences and people and how to manage and report any ethical matters back to the university and relevant statutory bodies. This foundation and self-awareness gave the researcher a sound basis on which to manage the collection, coding, indexing and interpretation of the research data, whilst simultaneously recognising the potential influence of values and bias on research design and analysis (Janesick, 1998) and that bias and value influences must be reflected on and hopefully minimised.

\subsection{Conclusion}

This chapter has sought to rationalise and contextualise the research's design, methodologies and methods. As discussed, the epistemological approach, the narrative inquiry methodology and the methods used were all central to addressing the knowledge gaps, which required gaining an understanding of the motivations of in-migrants and the perceptions of key actors. These research methods allowed the researcher to identify the key elements in the case study and to unpack the complex interactions between the physical environment, human social, cultural and economic capital and the town's image and governance landscape. This enabled the researcher to apply Lamarck's conceptualisation that changes to the physical environment should, in theory, cause people to learn about and adapt to that new environment, which in turn causes changes in the behaviour, 'personality' and image of the seaside town and its consequent regeneration or degeneration. This required an examination of these connections between the physical environment, middle-life migration and the policies of key agencies through the adopted research methods.

It is recognised, however, that narrative research does not strive to produce any conclusions of certainty but aims for its findings to be 'well-grounded' and 'supportable' and does not claim to represent the 'exact' truth but rather garner interpretations where elements of truth and reality exist. The validity of the findings was generated through sound and robust data collection. Flick (2006) emphasised that in qualitative research validity is underpinned from 
trustworthiness, credibility and authenticity of the data and this was achieved by the researcher using appropriate research methods, clearly outlined criteria, accepted procedures, systematic analysis, adequate discussion and a clear distinction between data and interpretation. The researcher having spent a considerable amount of time in the locality also gained a greater understanding of the culture and was able to facilitate participant involvement in the research from across the town and different backgrounds. This offered valuable insights and provided the 'convenience' and opportunity to overcome 'accessibility' constraints, access to information and political nuances. It also meant that individual participants were confident of the research findings that otherwise may have been viewed as problematic. It also enabled the researcher to become closely involved with the data collection, as this created human conditions that generated 'enough trust' for the participants to disclose their views on the subject under investigation. Questionnaires were piloted, language adapted, dress-code and behaviour were modified to suit the audience. Training in aspects of ethics and equalities facilitated transcripts of a high quality with all the participants having engaged fully. All interviews were recorded and were customarily of an hour duration in the participants own chosen setting that enabled them to feel relaxed and willing to participate. The longest interview was 76 minutes and on average interviews were 63 minutes. Every participant signed a meetings information sheet and confidentiality form prior to the commencement of the interview that positioned both the researcher and participant into a trusting engagement. The in-depth interviews were used to gain an insight into complex narratives, stories and emotions that needed to be understood as part of the narrative inquiry approach, where the researcher was able to guide the interview through the adoption of interview questions requiring a softer, subjective angle, allowing insight into issues of a unique and personal nature. Reflective practice and researcher diary notes were employed in an attempt to make explicit any issues of bias, deception or ethical concerns. Field notes were kept before, during and after the interviews and these were placed with the signed confidentiality forms, transcripts and interview tapes to enable the researcher to reflect on issues and which offered a clear audit trail. This validity of data through trustworthiness, credibility and authenticity was at the core of the research findings. The research design, methodologies and methods followed the approaches as promoted by Silverman (2001), Guba and 
Lincoln (2004), Flick (2006) of a thorough detailed account of the research process but understanding that narrative research conclusions generally remain open-ended (Webster and Mertova, 2007), which is the case for the findings of the research considered within Chapters 6, 7 and 8 . Also, the application of single case study research while offering in-depth, real-life context and phenomena in detail (Yin, 2009) also has limitations about making generalisations of the findings beyond the case study area. 


\section{Chapter 5}

\section{CASE STUDY SECONDARY DATA}

\subsection{Information}

The purpose of this chapter is to present the key findings from the documentary analysis of Bexhill-on-Sea, which will provide both the context to the analysis in Chapters 6 and 7 and also inform this analysis.

\subsection{Bexhill-on-Sea profile}

Bexhill-on-Sea is located in East Sussex on the southeast coast of England. The town is 55 miles from central London, 30 miles east of Brighton and five miles west of Hastings (Figure 5.1). The main issues in relation to transport are journey times and accessibility to jobs. These poor transport links are frequently cited as one of the main reasons contributing to economic disadvantage in this area (East Sussex County Council, 2008; RDC, 2011c). However, alongside poor transport links, other strategic documentation for Bexhill-on-Sea and Rother District Council (RDC) (the local authority area containing Bexhill-on-Sea) cites labour skills shortages as a key reason for the relatively poor economic performance for an area of southeast England (RDC, 2006a, 2011b, 2011c; SeaSpace, 2003a, 2003b; East Sussex County Council 2012).

Bexhill-on-Sea is the largest settlement in the local authority area of RDC, with a population of 43,531 (ESiF, 2010c) - equivalent to some 48 per cent of the district as a whole. The town has a high proportion of older people, with 36.5 per cent of the population aged over 65 years (RDC, 2011c), which compares with 23.5 per cent for East Sussex and 17.5 per cent for the southeast. Adding in the percentage of those under the age of 16 living in the town ( 14.5 per cent), this then equates to 51 per cent of Bexhill-on-Sea's population being outside of working age (ESiF, 2010c). Migration information outlined below confirms an increasing trend in older couples (aged 50+) and retirees moving to the town (DTZ/Pieda, 2006). 

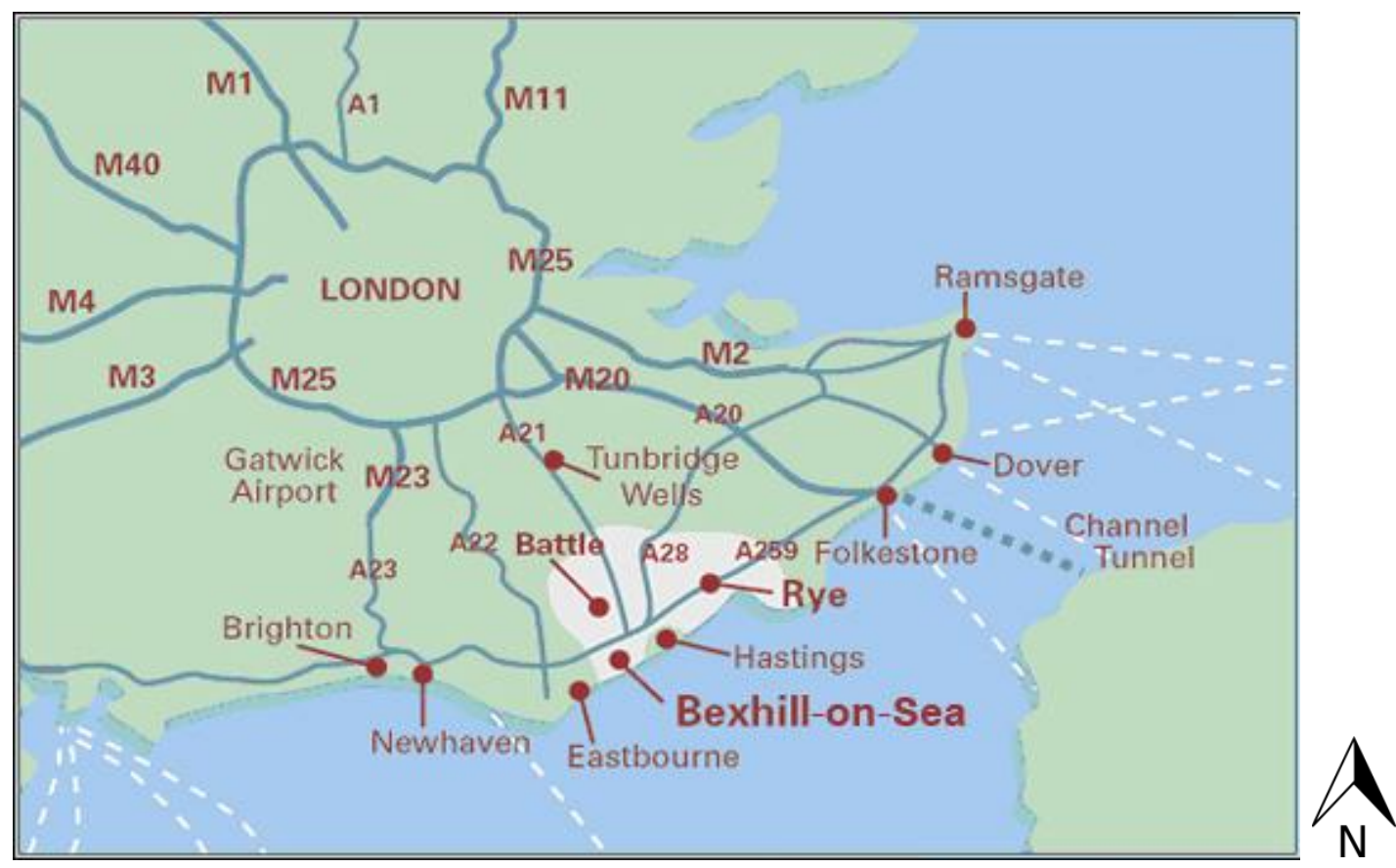

Figure: 5.1 Map of southeast England

Scale: $1 \mathrm{~cm}=14$ kilometres

'Source: Rother District Council, (2012)' (Original in colour)

Bexhill-on-Sea is often analysed together with its neighbouring seaside resort town of Hastings, but the documentary analysis revealed that the two towns are so distinctly different in their socio-economic, cultural and physical characteristics that they cannot be treated as a single resort destination. As Beatty and Fothergill (2003) explained in their report on the state of the British seaside economy, Bexhill-on-Sea is among those seaside towns that have significantly above average middle-lifers and retired populations and have changed their complexion from the traditional seaside resort as a result of the loss of seaside tourism market share with consequent negative impacts on the related infrastructure and image.

Bexhill-on-Sea, with its 'Costa Geriatrica' image (Shaw and Williams, 1997; McHugh, 2003; Argus, 2005, 2009), is rare, in that it has one of the highest concentrations of elderly people in England and is forecasted to continue to hold this position (Appendix 16a). Population data (defined by the urban census output areas aligned with local authority ward boundaries) show a significant spike in the later middle-life population (aged 60-69), which is further emphasised in the elderly categories aged $70+$ (Table 5.1). 


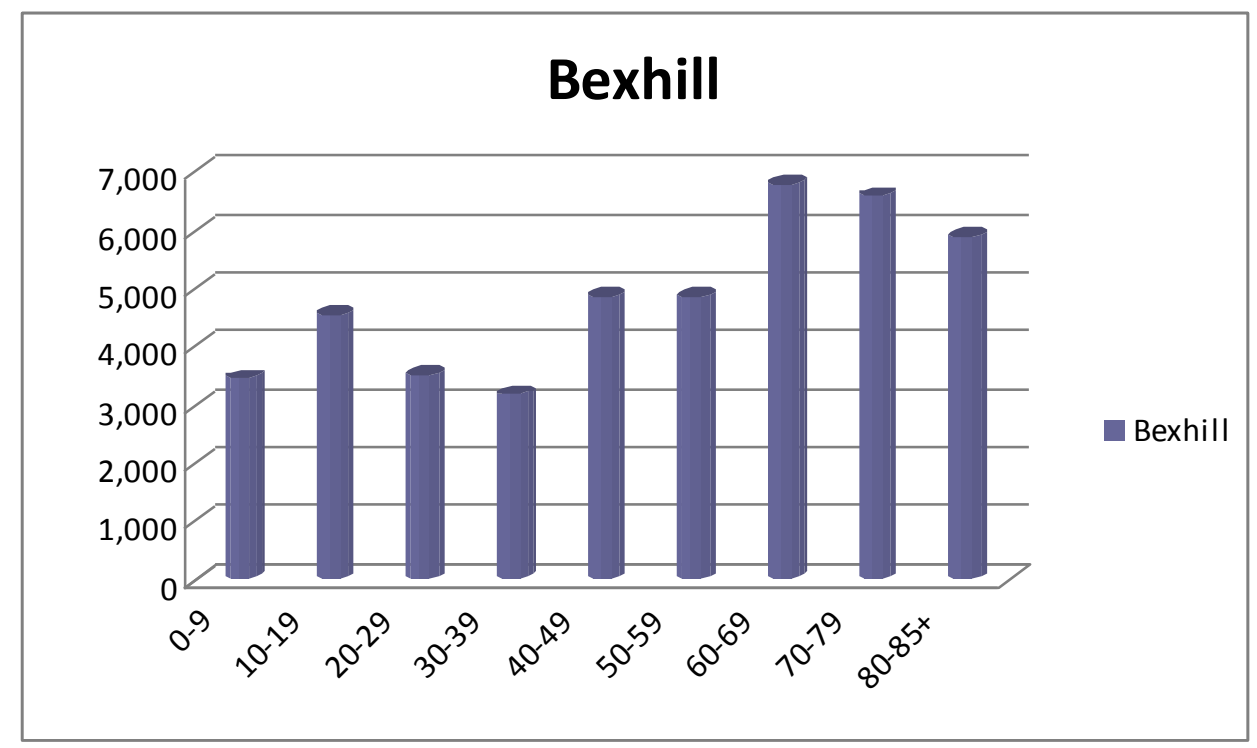

Table: 5.1 Bexhill-on-Sea populations by age group, 2010

'Source: East Sussex in Figures' (Original in colour)

When these population figures are converted to percentages (Table 5.2) and compared to the district of Rother, East Sussex (the contextual county), the southeast region of England and England as a whole, then Bexhill-on-Sea shows distinctive and rare social characteristics. The proportion of the population in the 'younger' middle-life age group (50-59) is similar to the county but is one percentage point below the average when compared to England and the southeast. For the later middle-life age group (60-69) the difference is more marked, with the proportion four to five percentage points above the regional and national averages. These higher proportions for later middle-lifers are also found for the percentage of the elderly population (70+), which is eight to nine percentage points above the regional and national averages. Linked to these data, Bexhill-on-Sea has a relatively small proportion of its population in the 2040 working age categories compared to the southeast and England.

With forecasted growth in an ageing population (Dunnell, 2001; ESif, 2010c; ONS 2010b, 2010c), due to people living longer, and the reasons as discussed in Chapter 3, this will most likely have further implications for the levels of middle-lifers and elderly in Bexhill-on-Sea. 


\begin{tabular}{|c|c|c|c|c|c|}
\hline \multicolumn{6}{|c|}{$\begin{array}{l}\text { Population by Age Group - mid-term estimates } 2010 \text { (actual and \% } \\
\text { age) }\end{array}$} \\
\hline Age Group & Bexhill & Rother & East Sussex & Southeast & England \\
\hline $0-9$ & 3,433 & 8,227 & 54,109 & 996,695 & $7,057,518$ \\
\hline $10-19$ & 4,523 & 10,398 & 61,699 & $1,039,007$ & $7,219,209$ \\
\hline $20-29$ & 3,493 & 6,475 & 47,211 & $1,067,080$ & $8,359,604$ \\
\hline $30-39$ & 3,155 & 6,943 & 50,235 & $1,088,451$ & $7,882,392$ \\
\hline $40-49$ & 4,835 & 12,055 & 74,967 & $1,283,858$ & $8,941,723$ \\
\hline $50-59$ & 4,854 & 12,130 & 68,840 & $1,044,017$ & $7,333,805$ \\
\hline $60-69$ & 6,771 & 14,513 & 70,727 & 934,470 & $6,496,051$ \\
\hline $70-79$ & 6,576 & 10,780 & 50,368 & 621,170 & $4,359,144$ \\
\hline $80-85+$ & 5,891 & 8,466 & 38,884 & 434,587 & $2,829,311$ \\
\hline Total & 43,531 & 89,987 & 517,040 & $8,509,335$ & $60,478,757$ \\
\hline Age Group & Bexhill (\%) & Rother (\%) & $\begin{array}{c}\text { East Sussex } \\
(\%)\end{array}$ & $\begin{array}{c}\text { Southeast } \\
(\%)\end{array}$ & England (\%) \\
\hline $0-9$ & 7.9 & 9.1 & 10.5 & 11.7 & 11.7 \\
\hline $10-19$ & 10.4 & 11.6 & 11.9 & 12.2 & 11.9 \\
\hline $20-29$ & 8.0 & 7.2 & 9.1 & 12.5 & 13.8 \\
\hline $30-39$ & 7.2 & 7.7 & 9.7 & 12.8 & 13.0 \\
\hline $40-49$ & 11.1 & 13.4 & 14.5 & 15.1 & 14.8 \\
\hline $50-59$ & 11.2 & 13.5 & 13.3 & 12.3 & 12.1 \\
\hline $60-69$ & 15.6 & 16.1 & 13.7 & 11.0 & 10.7 \\
\hline $70-79$ & 15.1 & 12.0 & 9.7 & 7.3 & 7.2 \\
\hline $80-85+$ & 13.5 & 9.4 & 7.5 & 5.1 & 4.7 \\
\hline Total & 100.0 & 100.0 & 100.0 & 100.0 & 100.0 \\
\hline
\end{tabular}

Table: 5.2 Population by age group - mid-term estimates 2010

'Source: ONS, (2001), mid-year estimates, East Sussex in Figures, (2010)'

\subsection{Foundations and early development of the resort town of Bexhill-on-Sea}

Bexhill-on-Sea has a number of similarities to many seaside resort towns, yet the town's past is not well-known compared to other, more popular seaside resorts. This is probably because its major development as a resort occurred very rapidly at the end of the nineteenth century, when it became the youngest borough in Sussex at its incorporation in 1902 (Porter, 2004). 
While there were occasional references to sea bathing throughout the $1700 \mathrm{~s}$, it was not until the eighteenth century that seaside resorts really began to develop (Walton, 1983). Most resort development was around established towns, but by the early nineteenth century resorts were being created in a number of ways, importantly initially led by entrepreneurs and landed gentry who were prepared to invest (Shaw and Williams, 1997; Brodie et al., 2005; Brodie and Winter, 2007). Resort functions were grafted on to existing towns, others were prepared to invest in entirely new developments and a third approach involved engulfing a pre-existing small coastal village, such as in the cases of Blackpool or Torquay. A fourth type of resort development, similar to the third type, was the creation of a new settlement built on coastlines, with small villages one or two miles in land. These were originally agricultural settlements aligned with the countryside rather than the coast, and they had established names that were often not adopted by the new resorts that began to engulf them (Brodie and Winter, 2007). Bexhill-on-Sea, East Sussex, is an example of this type of resort development.

'Bexelei' was a small, mile-inland farming community in which the De La Warr estate owned much of the surrounding land. In 1846, the railway came to, or rather past, the settlement, being some distance north of the line, which later sowed the seed for Bexhill's development from a village to a town (Bartley, 1971; Porter, 2004; Bexhill Museum, 2010b). It was the $7^{\text {th }}$ Earl (1817-1896) who was responsible for creating the new resort of Bexhill-on-Sea further down the hill from the Old Town into much of what we know today (Fairley, 2006). The $7^{\text {th }}$ Earl employed the building contractor John Webb to develop much of the town, and in part payment for his services he transferred to Webb a considerable portion of the land, much of what would be considered today as central Bexhill-on-Sea. Webb's section of Bexhill-on-Sea would include the majority of shops and services, whereas the De La Warr Estate, to the east, was to be made up of large houses and fine hotels. Between 1870 and 1900, Bexhill-on-Sea was transformed from a small farming village into a seaside resort town in which growth was neither natural nor gradual and which encouraged a rapid influx of new people into the area (Porter, 2004). 
While the $7^{\text {th }}$ Earl had started the development at the end of his life, the $8^{\text {th }}$ Earl (1869-1915) took over and extended the development. In 1902, Bexhill's seafront became the first place in Britain to host motor races, and by the turn of the century the foundations had been laid for Bexhill-on-Sea as an upmarket seaside resort for the fashionable, well-heeled society that the $7^{\text {th }}$ and $8^{\text {th }}$ Earls had wanted to attract (Bexhill Museum, 2010a; Fairley, 2006). The new resort of Bexhill-on-Sea gained a new railway station in 1902, appropriate to its growing size and ambitions. The station was remarkable for the length of its platforms, and it still stands as a testament to the vast numbers of people who came to the town during its heyday; thousands of well-to-do visitors would stay for weeks or months during the summer season and, as they were leaving, thousands of school children would be arriving to start the new term (Gray, 1994). Education had an important role in the development and character of Bexhill-on-Sea, with large numbers of young people attending independent schools (over 300 since the development of the resort) contributing to the town's economy (Gray, 1994; Porter, 2004). Many of the pupils came from families in colonial administration and the armed forces (Searson, 1998), who found the town a safe and healthy place for their children to be educated. Highclass tourism and independent education were the main sources of the town's prosperity (Porter, 2004).

\subsection{Social divisions within the town of Bexhill-on-Sea}

As in many communities there were social divisions, but historically within Bexhill-on-Sea these were particularly striking. Residents living on independent means seemed to find visitors to the resort an intrusion and, more importantly, these residents were not prepared to pay higher rates for facilities to attract visitors; anything that would cost the resident ratepayers money was viewed with the deepest suspicion (Stevens and Willis, 1990; Gray, 1994; Fairley, 2001). Tourism, of course, was as important to the shopkeepers and publicans on the Webb's estate as it was for the hoteliers on the De La Warr Parade, both cohorts being dependent on the rich visitors coming to the town and spending freely (Porter, 2004). 
In 1933, the $9^{\text {th }}$ Earl De La Warr, as mayor of Bexhill-on-Sea, acting as a 'municipal capitalist' (Russell and Faulkner, 2004), proposed a new plan for an entertainment centre (Fairley, 2001). The spectre of ratepayers' money being spent ignited opposition to the project. Hostility over the nationality of the architects, Mendelsohn (German) and Chermayeff (Russian), and the aesthetics of the design took second place to the underlying fear of the council spending public money on a facility for the use of visitors (Stevens and Willis, 1990). Nevertheless, the De La Warr Pavilion was completed in 1935, and whilst it is not Bexhill's only distinct building, it is certainly the best known (Brook, 1997). The national and international significance of the now Grade I listed building cannot be doubted, but it remains as controversial today as when it was first built (Brook, 1997; Fairley, 2006). However, it was quickly eclipsed by the Second World War, and whilst there were boom times for tourism in many resorts in the 1950s, for Bexhill-on-Sea many of the independent schools, on which the town was heavily reliant, did not return after the war, while those that did so gradually declined. It was probably the end of the British colonies and the independence of India in 1947 that led to the decline of the private schools, as many of the pupils had been left as boarders by parents working overseas (Searson, 1998; Porter, 2004). As advertising campaigns promoted the region's climate, its facilities and its particular brand of the 1930s idyll to visitors, so what Fairley (2006) calls the emergent 'Wealth of Health' coast developed a retirement belt, and it was towards Bexhill-on-Sea that some of this new migration headed (Fairley, 2006). Many who moved to the town were retired and on fixed incomes, and so, it appears, they had to be cautious with their spending (Porter, 2004; Fairley, 2006; Waite, 2009). The proportion of those residents of independent means began to outnumber those who worked in the tourist economy, and so it became politically more difficult for ratepayers' money to be used on facilities for visitors. Gradually the town declined as a resort and became more residential in character (Porter, 2004; Waite, 2009). This was characterised when, in 1960, Bexhill Corporation commissioned a promotional film, 'A Summer Memory of Bexhill' (Bexhill Museum, 2010a), where the last few minutes of the film addressed relocating to the town and not just visiting the town, as if admitting that it was no longer classed as a resort. 
These changing socio-economic conditions for Bexhill-on-Sea exhibit, on the surface, a number of similarities to other principal seaside resorts in England, where Shaw and Williams (1997), Walton (2000), Agarwal and Shaw (2007), Benedict and Houghton (2009), Walton and Browne (2010) identify a whole range of complex exogenous forces influencing English seaside resort capacity (Appendix 17). Understanding these multifaceted socio-economic factors that shaped the town's development is therefore explored in more detail next.

\subsection{Employment and economic status}

Beatty et al., (2008) note that employment in seaside towns is predominantly led by two sectors (Appendix 8), with 'distribution, hotels and restaurants' being the largest with a third of all jobs, comprising most tourist-related occupations. This sector, however, also includes all retailing, which is not necessarily contingent on tourism. The second most dominant type of employer in seaside towns, accounting for nearly a third of all jobs, relates to public administration, education and health. Both these sectors are well above the English average, with the other employment sectors falling well beneath the average. This high reliance on public sector jobs in seaside towns will be of major concern to community leaders over this next decade, as the government has committed to addressing the public deficit by cutting back on public services by 20-45 per cent (Cameron, 2009; CIPFA, 2009; CIPFA/Solace, 2009). In Hastings and Bexhill-on-Sea combined, the proportion of jobs in the public sector (43 per cent) is almost twice the national ( 26 per cent) and southeast ( 25 per cent) averages (Beatty et al., 2008). Furthermore, seaside economies are mostly low-wage, low-productivity economies, and while this is not well-documented in any systematic way, an examination of earnings statistics (Appendix 18) backs up this point. None of this is surprising; the tourist and public sectors are mostly lower paid sectors and seaside towns lack many of the well-paid jobs in, for example, banking and finances (Fothergill, 2007). However, a note of caution is required, as the earnings figures are for seaside town residents whereas commuting flows mean that the earnings from employment in the town itself will in some cases differ substantially (Shaw and Williams, 1997; Beatty et al., 2008). Furthermore, in England's principal seaside town employment grew prior to the 2008 downturn and is shown to be up by 7.5 per cent between 1998 
and 2006 compared to 6.9 per cent in England as a whole (Beatty and Fothergill, 2003), although much of the job growth in seaside towns is part-time in nature. However, while employment in these towns is growing, so too is the labour supply, which has been caused by in-migration and labour market imbalance to persist in some of the towns (Fothergill, 2007).

Table 5.3 reveals that seaside towns' working age populations would be in decline if it were not for in-migration.

\begin{tabular}{|c|c|c|c|c|}
\hline \multicolumn{5}{|c|}{$\begin{array}{l}\text { Labour market accounts for total working age population, English } \\
\text { seaside towns 1971-2001 }\end{array}$} \\
\hline & & & Numbers & $\begin{array}{l}\% 1971 \text { working } \\
\text { age population }\end{array}$ \\
\hline *1 & & Natural increase in workforce & $-90,000$ & -6.2 \\
\hline$\star^{2}$ & Plus & Net in-migration & 360,000 & 24.9 \\
\hline$\star^{3}$ & Plus & Increase in net in-commuting & $-21,000$ & -1.5 \\
\hline$\star^{4}$ & Plus & Increase in labour force participation & 88,000 & 6.1 \\
\hline$\star^{5}$ & Minus & Increase in employment & 317,000 & 22.0 \\
\hline$\star^{66}$ & Equals & Increase in recorded unemployment & 19,000 & 1.3 \\
\hline
\end{tabular}

Table: 5.3 Labour market accounts for total working age population, English seaside towns 1971-2001

'Source: Beatty and Fothergill, (2004)'

${ }^{*} 1$ in the absence of migration the population of working age in seaside towns is in decline

${ }^{*}$ net in-migration of men and women of working age has been substantial

${ }^{*} 3$ this figure is the net sum of the numbers in-commuting and out-commuting. Over the period as a whole, net in-commuting is estimated to have declined by 21,000

${ }^{*}$ this is the impact of the changing share of men and women of working age who are either in employment or recorded unemployment

${ }^{* 5}$ this shows the increase in the number of jobs located in seaside towns. A reasonable assumption might have been a reduction in line with the erosion of the traditional tourist base ${ }^{*}$ this figure accounts for the modest increase of 19,000 in recorded unemployment over the period as a whole

The first line shows that in the period 1971-2001 the decline of the working age population would have been about 6 per cent, or 90,000 (Beatty and Fothergill, 2004). Whereas net in-migration for seaside towns is the single largest flow in the labour market accounts (Beatty and Fothergill, 2004), this in-migration is not spread evenly across age bands, as illustrated in Appendix 10. Therefore, as 
Beatty and Fothergill $(2003,34)$ comment, 'small changes in recorded unemployment can hide a much bigger labour market flow and that changes in recorded unemployment are an unreliable guide to the relative economic strength of areas'.

The employment rate (which is the share of the working age population including self-employment - in work) in seaside towns is below the English average (72 per cent compared to 74 per cent in 2006), but there are considerable disparities between them, as presented in Appendix 19. However, whilst employment rates offer a useful indicator, employment rates alone give a false impression of the health of the seaside economy, as economically inactive students, who can be numerous and concentrated, can distort the picture (Appendix 20). With a few exceptions, notably Brighton and Bournemouth, seaside towns have few universities or higher educational institutions, which have the effect of boosting the employment rate compared to areas where students attend (Fothergill, 2007). There is also considered to be a substantial informal economy within seaside resorts that is not captured in this data (Ambrose, 2010). It is therefore possible that overall employment in seaside towns could mask a dependence on part-time jobs, whereby 38 per cent of jobs in seaside towns are part-time compared to 30 per cent across England as a whole (Beatty et al., 2008), as residents supplement their income through the informal economy.

\subsection{Benefit claimants}

Within the UK the principal group of non-employed working age benefit claimants are on incapacity benefits (Appendix 21). However, it appears that job losses, rather than deteriorating health, underpin much of the increase in incapacity benefit claimants since the 1970s (Alcock, 2003; Autor and Duggan, 2003; Beatty and Fothergill, 2007). Therefore, recent changes from incapacity benefit (DWP, 2010a) to what is now referred to as 'Employment and Support Allowance' means that the government is seeking to change this situation (DWP, 2001) and has introduced a 'Work Capability Assessment' of those on incapacity benefit (DWP, 2010b). The view of the government is that incapacity benefit claimant rates, which are disproportionately high in some parts of the 
country, have nothing to do with deficient demand for labour (DWP, 2003b). Their view is that 'there are plenty of jobs everywhere and that the lack of skills or motivation is the root cause' (Beatty and Fothergill, 2007, 138). A further benefit claimant category includes those claiming pension credits - around a quarter of seaside town residents over 60/65 draw on pension credits, which is much the same as the English average, but there are important differences between individual towns. Some of these differences are likely to reflect the scale of the influx of affluent retirees (Appendix 21). This is noticeable in the Hastings and Bexhill-on-Sea figures, as they are beneath the average for seaside towns and England as a whole, and yet Bexhill-on-Sea has proportionally one of the highest concentrations of elderly people (Beatty et al., 2008).

\subsection{Deprivation}

Of the 37 principal seaside resort towns, 26 have greater overall deprivation than the English average (i.e. a ranking below 16,242) per the criteria for the lower layer super output areas (LSOAs) prescribed by DCLG (2007b). Table 5.4 shows Penzance in the southwest as the most deprived seaside resort town, but this could be reflected partly in the town's unusual statistic of households without central heating. Hastings and Bexhill-on-Sea is the most deprived in the southeast at 11,666.

\subsection{Bexhill-on-Sea indices of multiple deprivation and employment}

Analysis of Bexhill-on-Sea's indices of multiple deprivation (IMD), using the datasets from the LSOAs provided in Appendix 22, reveals a slow decline in the town's socio-economic profile. In 2004, the IMD-combined LSOA score for Bexhill-on-Sea was 17,285, which was better than the English average ranking of 16,242 from a total ranking of 32,482 . By 2007 , this position had deteriorated to 16,114 - a drop of nearly 7 percentage points. By 2010 , the IMD score for the town had quickened its deterioration to 14,602 - a drop of over 9 percentage points on the previous period and over 15 percentage points within six years, thus placing the town in the lower half performance classification of the England average IMD. Each of the 28 LSOAs for the town have been 


\begin{tabular}{|c|c|c|c|c|c|}
\hline \multicolumn{6}{|c|}{$\begin{array}{l}\text { Average deprivation ranking - LSOAs in England's principal seaside towns, } \\
\text { 2007: average } 16,242\end{array}$} \\
\hline \multicolumn{2}{|c|}{ Most deprived } & \multicolumn{2}{|c|}{ More deprived } & \multicolumn{2}{|c|}{ Less deprived } \\
\hline Penzance & 6,758 & Ilfracombe & 9,190 & Burnham-on-Sea & 16,479 \\
\hline Skegness & 7,004 & Clacton & 9,193 & Southport & 16,505 \\
\hline \multirow[t]{21}{*}{ Yarmouth } & 7,898 & St. Ives & 10,980 & Deal & 16,646 \\
\hline & & Bridlington & 11,257 & Bournemouth & 17,097 \\
\hline & & Newquay & 11,424 & Bognor Regis & 17,122 \\
\hline & & Hastings and Bexhill & 11,666 & Greater Worthing & 17,548 \\
\hline & & Torbay & 11,720 & Exmouth & 18,372 \\
\hline & & Morecambe/Heysham & 11,732 & Whitstable/Herne Bay & 18,428 \\
\hline & & Thanet & 11,770 & Swanage & 18,980 \\
\hline & & Scarborough & 11,808 & Whitley Bay & 22,642 \\
\hline & & Lowestoft & 11,856 & Sidmouth & 23,464 \\
\hline & & Whitby & 12,253 & & \\
\hline & & Falmouth & 12,634 & & \\
\hline & & Blackpool & 12,719 & & \\
\hline & & Minehead & 12,773 & & \\
\hline & & Brighton & 12,938 & & \\
\hline & & Eastbourne & 13,236 & & \\
\hline & & Folkestone and Hythe & 13,733 & & \\
\hline & & Dawlish/ Teignmouth & 14,430 & & \\
\hline & & Isle of Wight & 14,481 & & \\
\hline & & Western-super-Mare & 14,817 & & \\
\hline & & Southend & 15,039 & & \\
\hline & & Weymouth & 15,644 & & \\
\hline
\end{tabular}

Table: 5.4 Average deprivation ranking - LSOAs in England's principal seaside towns, 2007 'Source: DCLG Indices of Deprivation (2007b), adapted from Beatty, et al., (2008)'

i) The most deprived wards in England are below 8,000.

ii) English average 16,242: below this figure means an above average level of deprivation iii) The indices of deprivation are assessed through seven domains: employment (22.5\%); income $(22.5 \%)$; education and training (13.5\%); health and disability $(13.5 \%)$; barriers to housing and access to services (9.3\%); living environment (9.3\%) and crime (9.3\%).

analysed against the seven domains that make up the indices. Appendix 23 provides a detailed breakdown of the neighbourhood LSOA rankings and (Table 5.5) provides a summary of the key issues, which informs the town's deprivation 
score. The first noticeable factor is the diversity between the LSOAs where ten of them (008A, 009A, 009C, 009D, 009F, 010A, 010B, 010D, 010E, 011D) have very limited levels of deprivation. At the other extreme, four LSOAs (007D, 007E, 011C, 011E) are in the worst 20 per cent of deprived areas in the country, with a further six that are approaching or within reach of the bottom 20 per cent quintile.

\begin{tabular}{|c|c|c|c|}
\hline \multicolumn{4}{|c|}{ Indices of Multiple Deprivation at LSOA for Bexhill-on-Sea } \\
\hline \multicolumn{3}{|c|}{$\begin{aligned} 1 & =\text { worst } \\
6,496 & =\text { worst } 20 \% \text { break } \\
16,242 & =\text { mid-way point } \\
32,482 & =\text { best }\end{aligned}$} & $\begin{array}{l}\text { employment } 22.5 \% \text {; income } 22.5 \% \text {; } \\
\text { education and training } 13.5 \% \text {; health and } \\
\text { disability } 13.5 \% \text {; barriers to housing, access } \\
\text { to services } 9.3 \% \text {; living environment } 9.3 \% \text {; }\end{array}$ \\
\hline $\begin{array}{l}\text { LSOA } \\
\text { Ref }\end{array}$ & $\begin{array}{l}\text { IMD } \\
\text { score }\end{array}$ & Ward & Key Domain Issues \\
\hline 007E & 1,259 & Sidley & $\begin{array}{l}\text { Employment, Income, Education, Health, } \\
\text { Crime, Living Environment }\end{array}$ \\
\hline 007D & 3,126 & Sidley & $\begin{array}{l}\text { Employment, Income, Education, Health, } \\
\text { Crime, Living Environment }\end{array}$ \\
\hline $011 \mathrm{C}$ & 4,600 & Central & $\begin{array}{l}\text { Employment, Income, Education, Health, } \\
\text { Crime, Living Environment }\end{array}$ \\
\hline $011 \mathrm{E}$ & 5,980 & Sackville & Employment, Health, Living Environment \\
\hline 011B & 7,409 & Central & $\begin{array}{l}\text { Employment, Income, Health, Crime, Living } \\
\text { Environment }\end{array}$ \\
\hline $007 \mathrm{~F}$ & 7,865 & Sidley & Employment, Income Education, \\
\hline $011 \mathrm{~A}$ & 8,035 & Central & $\begin{array}{l}\text { Employment, Income, Health, Living } \\
\text { Environment }\end{array}$ \\
\hline 007C & 8,467 & St Stephens & Employment, Income, Education \\
\hline 007A & 8,614 & Old Town & Employment, Income, Health, Housing \\
\hline 008D & 8,990 & St Michaels & Employment, Education, Health \\
\hline $008 \mathrm{C}$ & 9,358 & St Michaels & Employment, Health \\
\hline 009G & 10,162 & Sidley & Employment, Health, Housing \\
\hline 011D & 11,618 & Sackville & None \\
\hline 008B & 13,405 & Sackville & Housing \\
\hline 007B & 14,010 & Old Town & Education \\
\hline 008E & 15,743 & St Michaels & Education, Health, \\
\hline 010D & 16,951 & Kewhurst & None \\
\hline $010 \mathrm{E}$ & 18,117 & St Stephens & None \\
\hline $010 \mathrm{~A}$ & 18,854 & Collington & None \\
\hline 010B & 19,516 & Kewhurst & None \\
\hline 009A & 22,106 & Collington & None \\
\hline $009 \mathrm{~F}$ & 22,490 & St Stephens & None \\
\hline 009E & 23,036 & St Marks & Housing \\
\hline $008 \mathrm{~A}$ & 23,726 & Old Town & None \\
\hline 010C & 24,816 & Kewhurst & Housing \\
\hline 009C & 25,668 & St Marks & None \\
\hline 009B & 27,295 & Collington & Housing \\
\hline 009D & 27,446 & St Marks & None \\
\hline
\end{tabular}

Table: 5.5 Indices of multiple deprivation at LSOA for Bexhill-on-Sea

'Source: East Sussex in Figures (2012); ONS (2012)' 
This deprivation is concentrated particularly within the town centre and the Central and Sackville wards, where there is a preponderance of Edwardian buildings that in many cases have been converted to HMOs or flatted dwellings (RDC, 2007a, 2010), and the Sidley wards, where there is a concentration of the youngest age group (0-15 years) that reside on a suburban estate, predominantly made up of social housing.

Analysis of the IMD data identifies that there are four reasons that contribute extensively to Bexhill-on-Sea's deprivation scores. These are employment, income, education and health (Appendix 23), though employment and income feature as the dominant factors across the majority of LSOAs which have deprivation scoring. This IMD quantitative analysis builds on other socioeconomic data as presented by Beatty and Fothergill (2008) and offers an indepth understanding of the social and economic profile of the town; however, it does not offer an interpretation as to why this profile exists and the influences that have seen the IMD data for Bexhill-on-Sea decline.

The factors causing change in seaside towns tend to be complex and multidimensional, with no one single factor that encapsulates the full range or extent of disadvantage (Agarwal and Shaw, 2007; Beatty et al., 2008; Beatty et al., 2009). In-migration has been recognised as a contributing cause of these problems, and Shaw and Coles $(2007,47)$ describe that 'such mobility flows are reshaping the social ecology of seaside resort towns and creating much more divided communities as well as a series of difficulties'. In this context, a number of intertwining problems can be recognised linked to in-migration; firstly, the profile of in-migration shows both a large proportion of retired people, who together with the low-skilled migrants put increased pressure on social care facilities (DCLG, 2007c), secondly, it has produced a disparity amidst the 'affordability ratio of earnings against house prices' (ODPM, 2006c) and thirdly, past periods of low investment in terms of the residential environment have produced a situation where, on average, resorts have over 50 per cent of their housing stock classified as 'non-decent' or 'unfit' (DCLG, 2010h). Poor housing conditions tend to be exacerbated by the higher level of private renting (on average 20 per cent), which is almost twice the rate as elsewhere (ODPM, 2006c). However, there appears to be no one cause to seaside economic 
performance, and while exogenous forces appear to have been significant, as shown in the analysis chapters, endogenous factors have also contributed to changes in the social and economic profile of Bexhill-on-Sea.

\subsection{Household migration flows: Rother District and Hastings Borough}

With in-migration and housing recognised in the review chapters as contributing to the decline of seaside resort towns, this section presents a documentary analysis for Bexhill-on-Sea. In May 2004, DTZ/Pieda Consulting was commissioned to undertake a study of sub-regional housing markets across the whole of the southeast region by the Southeast England Regional Assembly and Regional Housing Board. Hastings borough and much of the rest of RDC were treated as one sub-region and exhibited significant cross-border household and labour force movements, with the two districts mapping onto the 'travel to work' area for Hastings (DTZ/Pieda, 2006). The analysis supports earlier studies, in that the sub-region demonstrates a high degree of selfcontainment in its household movement patterns (DTZ/Pieda, 2006, 2010) and reveals strong, localised housing markets centred upon Hastings and Bexhillon-Sea with only a limited amount of movement evident between them. Movements from Hastings into rural Rother are much less in evidence within the mapping, a pattern that is likely to reflect the higher house prices compared with Hastings and the restriction to household mobility which this engenders (RDC, 2006a, 2007a, 2010). Overall, both Hastings and Bexhill-on-Sea are the recipients of a high number of long-distance inward migration movements, with a notable proportion of these originating in Sevenoaks and south London (DTZ/Pieda, 2006).

The migration data presented in Figure 5.2 analysed the addresses of households at the time of the 2001 Census, and also one year previously, and so present the number of households moving in one year. The figure reveals net in-migration into Hastings and Rother of 920 households in the year preceding the 2001 Census, which represents 1.2 per cent of all households in the area. Net in-flow is the result of an in-flow of 2,850 households combined with an out-flow of 1,930 households. A further 3,820 households moved within Hastings and Rother, amounting to 5.05 per cent of the total population. This 
implies a significant movement of households, both within the districts and migrant flows into the sub-region, and if maintained year on year, this represents a large demand for additional housing (DTZ/Pieda, 2006).

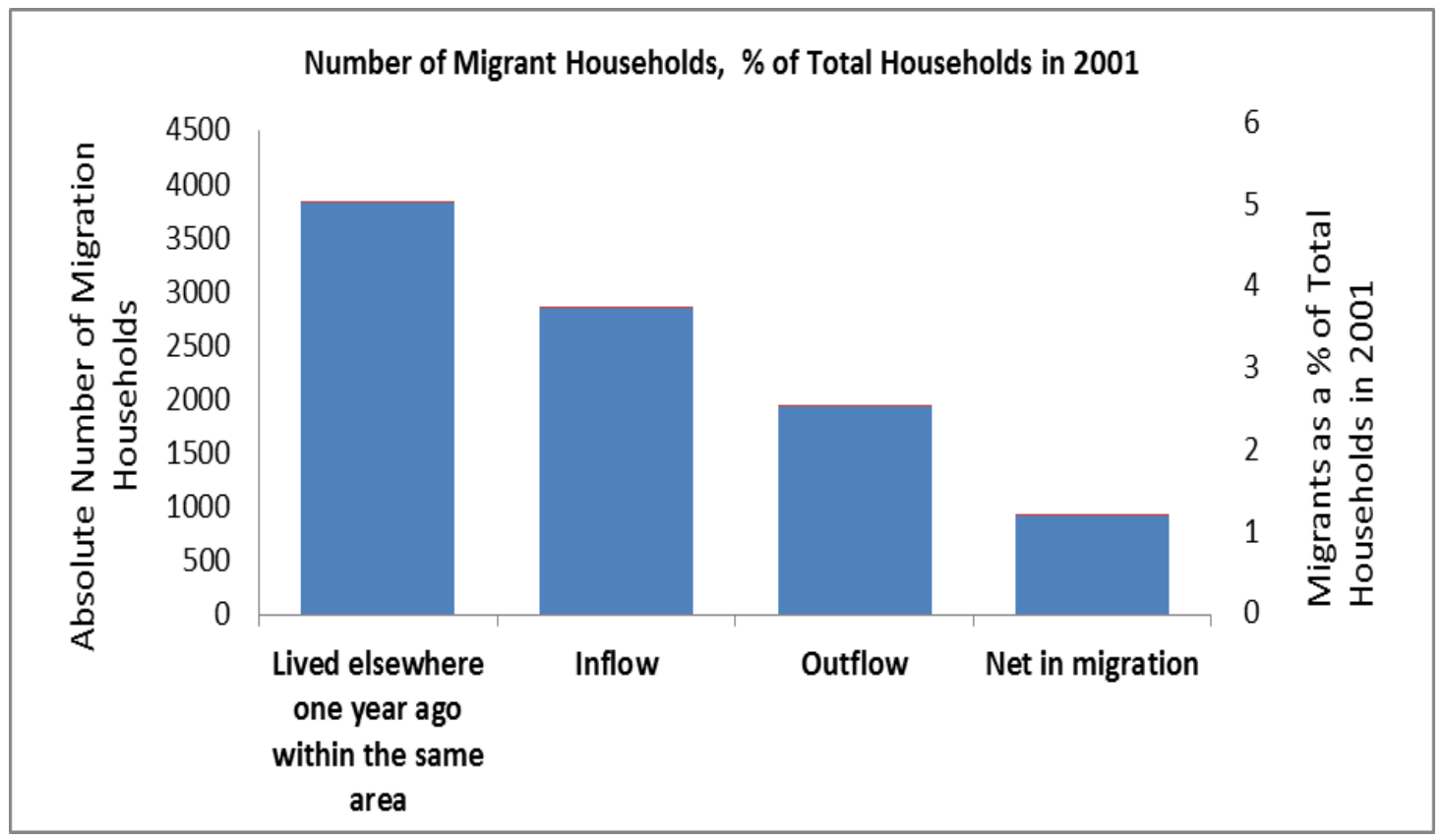

Figure: 5.2 Hastings and Rother household migration flows; year preceding 2001 census 'Source: Census (2001), taken from DTZ/Pieda, (2006) copyright Maplnfo/Bartholomew (2000)'. (Original in colour)

Table 5.6 shows migration by age group during the 2000 decade and reveals a composition of in-migrants and out-migrants that, on the whole, supports previous studies by DTZ/Pieda (2006), in that there is a consistent out-migration of the younger working age population and a larger proportion of in-migration in the middle-life age brackets. The demographic ageing of Rother is particularly evident (Appendix 16b), even though this has been a trend of many of England's seaside towns (especially on the south coast). However, the 2031 projections for England (Appendix 16c), when compared to the seaside resort of Bexhill-on-Sea, reveal an even more pronounced effect of the future ageing population on the town, whereby the traditional 'bell shape' population curve is replaced by an upside down pyramid (Appendix 16a). 


\begin{tabular}{|l|c|c|c|c|c|c|}
\hline \multicolumn{7}{|l|}{ Migration by Age Group in Rother District, 2001-2011 } \\
\cline { 1 - 4 } Age Group & All people & $0-14$ & $15-24$ & $25-44$ & $45-64$ & $65+$ \\
\cline { 1 - 4 } Year & & & & & & \\
\hline $2001-02$ & 1,600 & 400 & -300 & 500 & 700 & 300 \\
\hline $2002-03$ & 1,200 & 200 & -300 & 400 & 600 & 300 \\
\hline $2003-04$ & 1,300 & 400 & -300 & 300 & 600 & 200 \\
\hline $2004-05$ & 1,000 & 200 & -300 & 300 & 500 & 200 \\
\hline $2005-06$ & 1,100 & 300 & -300 & 300 & 500 & 200 \\
\hline $2006-07$ & 1,700 & 400 & -200 & 400 & 800 & 300 \\
\hline $2007-08$ & 1,300 & 300 & -200 & 400 & 700 & 200 \\
\hline $2008-09$ & 800 & 300 & -300 & 200 & 500 & 100 \\
\hline $2009-10$ & 1,000 & 300 & -400 & 400 & 600 & 200 \\
\hline $1010-11$ & 600 & 200 & -400 & 200 & 400 & 100 \\
\hline
\end{tabular}

Table: 5.6 Migration by age group in Rother district, 2001-2011

'Source: ESif East Sussex County Council, (2012)'

The significance of this relates to the earlier description of demographic changes and the country's total dependency ratios (DWP, 2009a). Table 5.7 projects for East Sussex County and RDC the total dependent ratio changes that are anticipated for the period 2006-2026, given current forecasts of planned housing development and population profiles across the county (ESiF, 2010b). Therefore, productive capacity in Rother and the total dependency ratio are forecasted as low as almost 1:1, whereas fifty years ago this ratio for the country was above 2:1 (Pensions Commission, 2004; DWP, 2009a). The implications are that the productive workforce in this area will fall in number and, in particular for Bexhill-on-Sea, this ratio may tip the balance between the numbers of those in work to those out of work.

\begin{tabular}{|l|c|c|c|c|c|c|}
\hline Dependency Ratios for East Sussex \\
\hline $\begin{array}{l}\text { Numbers and } \\
\text { Ratios }\end{array}$ & \multicolumn{2}{|c|}{$\begin{array}{c}\text { Dependency ratios } \\
\text { all dependants } \\
\text { (elderly and young) }\end{array}$} & \multicolumn{2}{|c|}{$\begin{array}{c}\text { Total working age } \\
\text { population }\end{array}$} & \multicolumn{2}{c|}{$\begin{array}{c}\text { Total elderly and } \\
\text { young dependents }\end{array}$} \\
\hline Year & $\mathbf{2 0 0 6}$ & $\mathbf{2 0 2 6}$ & $\mathbf{2 0 0 6}$ & $\mathbf{2 0 2 6}$ & $\mathbf{2 0 0 6}$ & $\mathbf{2 0 2 6}$ \\
\hline East Sussex & 68 & 86 & 301,456 & 290,667 & 204,767 & 248,690 \\
\hline Rother District & 80 & 94 & 48,704 & 49,634 & 38,860 & 46,612 \\
\hline
\end{tabular}

Table: 5.7 Dependency ratios for East Sussex

'Source: East Sussex in Figures, (2010)' 
The literature review identified that in-migrating pre-retirees and retirees have had a significant impact on seaside towns. An examination of the documentary data on Bexhill-on-Sea supports this analysis of the importance of retirement migrants and their socio-economic and cultural impact, and how this may be linked to deprivation in the town. It appears that their socio-economic and cultural backgrounds and behaviours can affect destinations, so it is therefore necessary to consider in more detail the characteristics of the middle-life age group, their mobility patterns and, importantly, likely future behaviours that may affect seaside resort town regeneration.

\subsection{Hastings and Bexhill-on-Sea regeneration}

The previous chapters reviewed the literature on seaside resort towns and identified how key agencies can contribute to and influence the socio-economic characteristics of these destinations. Consequently, actions taken by the key agencies over the past twenty years in Bexhill-on-Sea are analysed in this section.

In very recent times, local authorities, such as RDC in East Sussex, have responded to the regeneration challenges of stagnation and decline in their seaside towns, by implementing a range of responsive measures designed to rejuvenate resorts. However, these investments may have been driven mainly by financial incentives as opposed to strategic planning - often aided by external funding opportunities such as the Lottery, European grants, private finance initiatives (PFIs), public private partnerships (PPPs) and single regeneration budgets (SRB), in order to construct year-round facilities, refurbish and conserve the historic built environment and refurbish accommodation in the hope of attracting visitors back to the area (Benedict and Houghton, 2009). Historically, many seaside towns' development came about from creative entrepreneurial landed gentry, business individuals or municipal capitalists (Morgan, 1992; Shaw and Williams, 1997, 2002; Walton, 2000, 2009; Russell and Faulkner, 2004; Russell, 2006a). Today's investors are more likely to be the large business corporations, such as supermarkets, or pension fund holders, pursuing regeneration based on urban and retail-led commercial 
returns, which appear to exclude the entrepreneurial spirit and creativity of past seaside investments (Urry, 1994; Hearn, 2008, 2010; CABE, 2010). With the Coalition government pursuing private sector-led regeneration (RDA, 2010; DCLG 2010a), the development of urban centres will likely depend on thriving property and consumer markets, so any downturn in the property market will directly affect the retail sector and dampen down the regeneration impact (Tallon, 2010). This has proved to be a challenge for many of England's principal seaside towns, as property values are lower than the national average (DTZ/Pieda, 2006; DTZ, 2010; RDC, 2007a, 2010; Beatty et al., 2008; Hearn, 2010) and retail rental values are so low that makes it financially unviable for the large multiples and key development partners to invest, thus creating a stagnating environment (Hearn 2010).

It was in 2002 that the Hastings and Bexhill Taskforce was established, through which the regeneration company 'Hastings and Bexhill Renaissance Ltd' (trading as SeaSpace - now SeaChange) operates and has received £57m of government grants to implement its 'Five-Point Plan' (SeaSpace 2003a, 2003b). The 'Five-Point Plan' addresses the following themes: urban renaissance, excellence in education, business development, transport improvements and broadband connectivity. To date there has been the completion of a new university; further education (FE) college developments; new office accommodation within the town centre of Hastings (SeaSpace, 2010b); the development of incubator and start-up business centres focused on the creative media industry (SeaSpace, 2010a); the 'environmental business corridor' on the local authority boundaries of Hastings and Rother and the gentrification of a new millennium community (SEEDA, 2008). Bexhill-on-Sea has had a new FE college built, and a $£ 38 \mathrm{~m}$ high school 'Building Schools for Future' programme opened in 2010 and is also seeking to attract 500,000sq.ft of new commercial development space pending the construction of a link road between the two towns (East Sussex County Council, 2009, 2010a; Department for Transport, 2012). The ten-year plan (amounting to $£ 341 \mathrm{~m}$ investment) sees the majority of funding coming from the private sector, but in the first three years significant investments were made by government resources to start the programme and to stimulate and attract private investors. The regeneration strategy for the Hastings and Bexhill Taskforce's 'Five Point Plan' (SeaSpace, 2003b) promotes 
urban regeneration and endorses regeneration as being more sustainable, if it promotes vitality through the activities and diversity of industry (Hamnett, 2003; Lees, 2008; Lees et al., 2008) that hopefully brings environmental and economic benefits (Tiesdell et al., 1996; Coupland, 1997; SeaSpace, 2003a, 2003b; Bailey et al., 2006; Roberts, 2007; Evans and Foord, 2009). Stead and Hoppenbrouwer (2004) discuss sustainable development frameworks and argue that diverse economic environments benefit the resident community as much as the visitor, as they promote urban renaissance. This recognition of diversity and the creation of a varied urban environment, in co-existence with tourism, is the approach adopted by the Hastings and Bexhill-on-Sea Taskforce (SeaSpace, 2003a).

Running in parallel with the Taskforce and the Economic Alliance is RDC's own capital investment programme for public infrastructure in Bexhill-on-Sea. Their corporate plan (RDC, 2006b) and regeneration brochure, 'Investment Opportunities in Bexhill-on-Sea' (RDC, 2007b), set out a number of key investment plans. Central for Bexhill-on-Sea is the emphasis on cultural regeneration by way of the $£ 10 \mathrm{~m}$ cultural flagship project, the seafront $\mathrm{De} \mathrm{La}$ Warr Pavilion, to stimulate new economic sectors (De La Warr Pavilion, 2007) such as the arts and cultural industries, thus providing a strong sense of identity (CABE/English Heritage, 2003; RDC, 2003; 2006b; English Heritage, 2007; English Heritage and Urban Practitioners, 2007; Deloitte and Oxford Economics, 2010; Heritage Lottery Fund, 2010). This investment is supported by other cultural investments in the town, such as English Heritage, the 'Heritage Economic Regeneration Scheme's' £670k (HERs) in support of private sector shop front improvements; the $£ 2.5 \mathrm{~m}$ re-development of the derelict Sidley railway goods yard, providing a new $50,000 \mathrm{~m}^{2}$ employment site in the heart of the town's deprived wards for new start-up businesses (funded by an RDC capital programme and an area-based grant); the $£ 1 \mathrm{~m}$ commencement of the refurbishment of Egerton Park (funded by RDC and European funding) and the $£ 5 \mathrm{~m}$ refurbishment of the West Parade seafront and the Colonnade 'Next Wave' project (£4.1m from RDC and £1m from the CABE SeaChange programme) to complement the $£ 2 m$ new extension and refurbishment investment in the Bexhill Museum ( $11 \mathrm{~m}$ RDC and $£ 1 \mathrm{~m}$ Heritage Lottery Fund) (RDC, 2006b, 2007b, 2011b). 
These cultural 'top-down' investments have been met with a mixture of support and criticism, and they go some way to sustaining the findings of socioeconomic and cultural studies that could argue such regeneration approaches either foster social polarisation, gentrification, social inequality and exclusion (Swarbrooke, 2000; Palmer, 2002; Shaw and Williams, 2002; Garcia, 2004; Miles, 2005; Smith, M.K., 2007), or that these approaches contribute to cultural inclusion, diversity and increasing community quality of life (Booth and Boyle, 1993; Smith, M.K., 2003, 2007; De La Warr Pavilion, 2007). In the case of Bexhill-on-Sea, the opposition to this cultural and urban regeneration may, however, be more aligned to the personality and demographics of the town as a result of its high preponderance of older people, who tend to be more averse to change (DWP, 2005; Atterton, 2006; Florida, 2008; Evans, 2009a), as evidenced by the $£ 5 \mathrm{~m}$ seafront public realm investment that has been met by strong opposition from a proportion of residents. A regular letter writer in the local paper stated "My pension is threatened, my carefully accrued savings have suffered blows of seismic proportions, my spending is reduced and my quality of life is under siege. And yet my elected members, apparently without a care in the world, are spending our reserves on the Next Wave project without even asking us if we want it" (Bexhill Observer, 4 September, 2010).

This top-down approach taken by SeaSpace, through mixed-use intervention, appears to have had a greater impact on physical improvements and some job creation in the area than the 'bottom-up' Hastings Borough Council Neighbourhood Renewal Funds (NRFs) and Area-based Grants (ABGs) and RDC's Single Regeneration Budget (SRB) and Area Investment Framework (AiF) programmes, as interpreted through economic indicators (NEF, 2007; Adams-Acton, 2010). These 'bottom-up' regeneration programmes have been targeted principally at community and neighbourhood improvements, with decision-making on the allocation of funds actioned through a local consortium of public, private and community businesses (RDC, 2011b).

However, while recent investment in Hastings and Bexhill-on-Sea, as evidenced through research by Church et al., (2007), has been linked to growth in the tourism sector and evolving creative industries, cultural assets and activities, 
the underlying problem for the seaside town is still a broad lack of local enterprise (Church et al., 2007). The towns are heavily reliant on public funding and there is a distinct lack of local enterprise, commercial dynamism (Church et al., 2007; Beatty et al., 2008) and diversity in other employment sectors (Fothergill, 2007; Beatty et al., 2008). The local economy, for the most part, is stagnant, and businesses find it difficult to sustain themselves, in that there is insufficient local custom and an underdeveloped consumption culture in the towns (Hearn, 2008, 2010). This therefore makes Hastings and Bexhill-onSea's economy partly dependent on their housing economy, where those who can benefit from the lower-cost house prices look to carry on their business in other, more vibrant economies (Church et al., 2007).

The past few decades of 'bottom-up' social neighbourhood and 'top-down' economic regeneration government programmes have raised questions about their ability to revitalise communities. Some authors on regeneration (McCarthy, 1995; Smith, M.K., 2003, 2007; Harvey, 2005; Evans 2009b, 2009c) believe the approach of supporting cultural flagships, or cultural regeneration through local entrepreneurship, building and nurturing local clusters, developing arts, creative and cultural industries, supporting local festivals and tourism, is the way to attract and retain talent, whereas other authors and regeneration practitioners (SeaSpace, 2003a; Stead and Hoppenbrouwer, 2004) argue for initiatives that diversify economies as a means of disrupting stagnation and decline and creating the right environment to attract in-migration of the 'right sort' of groups of people and businesses. Other literature suggests that the physical environment requires changing if it is to modify the demographics and attract the creative people, innovators and 'open-to-experience' personality types, in order to regenerate seaside towns. In today's economy, talent, innovation and creativity are not distributed evenly (Florida, 2004, 2008; Florida and Timagli, 2004; Florida and Mellander, 2007) - they are concentrated in specific locations, and these clusters create forces that make places more productive and attractive, thus generating increases in output and wealth (Florida, 2007). 


\subsection{Conclusion}

The literature review, along with the documentary research data, shows how the physical environment of Bexhill-on-Sea has transformed from a tourism resort to a retirement town by the sea through the alteration of commercial property and the addition of property for housing the elderly. In turn, this has also been 'shaped' partly by the migration of a different set of socio-economic and cultural activities that have also changed the image of Bexhill-on-Sea, with the town's main function being to 'house' an elderly population in a seaside environment that offers 'some services'. The town supports a local business economy that 'serves' an ageing demographic with limited spending power, who over the decades have come to predominantly occupy and set the personality of the place. This gradual process of change, from a seaside resort to a retirement town by the sea, has been accompanied by slow decline in socio-economic performance and an increase in deprivation. The environment, demographics and images that are attached to the resort will be drawn out over the following two chapters. 


\section{Chapter 6}

\section{ANALYSIS - PHYSICAL ENVIRONMENT AND AGENCY}

\subsection{Introduction}

This and the following chapter address the research questions by presenting the findings from the primary research. Knowledge gaps identified for further research were, firstly, to analyse the key agencies of change and how they perceive their attempts to influence the physical environment, and secondly, the effects of these changes. This chapter analyses these past and current perceptions of participants, particularly key agencies, and how the actors perceive the town has evolved and the changes to its physical environment, human socio-economic and cultural capital, image and economic performance. These points are explored through the examination of participant narratives.

This chapter is broken down into four parts. Firstly, it analyses perceptions of Bexhill-on-Sea in the context of the environment and explores the participants' perceptions of the town. Secondly, this analysis of the environment and how it has changed, as well as participant perceptions, is understood in relation to the impact on employment and the local economy. Thirdly, the chapter looks at understanding the role and impact of key agencies, and in particular the governance of Bexhill-on-Sea, and how their urban planning measures and governance policies have impacted on the environment and demographics. Finally, the chapter seeks to analyse recent attempts made by the local authority to disrupt the environment through its leadership role, and concludes by presenting the emerging complex picture in Bexhill-on-Sea.

\subsection{Participants' perceptions of Bexhill-on-Sea's environment}

Chapter 4 (Tables 4.1 and 4.2) lists the key agency and middle-life participants $^{1}$. In terms of their perceptions about the town, a consistent

\footnotetext{
${ }^{1}$ If a quote commences with the code 1, this represents a middle-lifer with their age category ending in the letter (a) 50-55; (b) 56-60; (c) 61-65; (d) 66-70. If the code starts with a 2, this represents a key statutory agent, while a code 3 represents a non-statutory key agent.
} 
narrative emerged throughout that it is essentially a place for older people, in that it has a slower pace of life than a city or cosmopolitan place and is seen as safe, quiet, clean, tranquil, genteel, non-vibrant and is frequently summed up as typically 'traditionally British':

Very friendly, it's got that safe feel about it, the area... very laid back genteel little seaside town... everywhere is clean (Ref 103a)

It has a particular attraction for those people either approaching or entering retirement age. It's also got a safe environment-it's perceived as having a safe environment as well by the sea (Ref 203)

The participants' perceptions of the place seemed to arise in part from their views of the people who lived in the town. They related this to the townscape, which resembled Florida's $(2004,2007,2008)$ notion that places, like people, have personalities and that the participants felt that they belonged in the town. A number of participants referred to a sense of traditional values that came from the older community and gave it a feel of genteelness and society belonging:

Well, it feels like a community, it's got a community feel to it. It's a bit old-fashioned, a bit quirky (Ref 119b)

There's a good community spirit... we have got St. Barnaby's Church on the corner in Sea Road and the vicar... is working actively to try and open up and encourage the community to realise that whilst it's a church, it's a facility for everybody in Bexhill (Ref 309)

I like the people. If you go in shops they are friendly, next door neighbours, very nice. You know, I've not met anybody I dislike (Ref 101c)

Their perceptions were also partly based on the presentation of the environment, which they separated out into the natural (all living and non-living things occurring naturally on earth) and the physical (human-made built and urban) environment. Their accounts, in most cases, were based on the town's 
physical environment, which received varied comments. On the occasions where the natural environment was discussed, this received very favourable comments. Many participants cited the town's coastline, the clean air and its close proximity to the countryside with plenty of parks and open spaces to enjoy, as commented by these middle-lifers:

I prefer to live in the country, and Bexhill is near enough to the countryside to be easily there (Ref 107d)

I thought it was very clean and I absolutely loved being by the sea. It was seen to be such wonderfully fresh air, almost too fresh sometimes! (Ref 108d)

Whilst the natural environment came through as a key attribute for the town, there were mixed views and opinions from the participants about the physical environment. The descriptions offered about Bexhill-on-Sea's built and urban setting tended to focus on the town centre, residential property, the loss of employment sites, the Grade I listed De La Warr Pavilion and the seafront promenade, which at the time of the interviews was in the process of being refurbished by the local authority. Comments concerning the physical environment were varied, with some seeing the town in good condition and suitable for their needs, while to a lesser extent others stated it was limited in its offerings, tired, lacked vibrancy and was in need of attention. Many of the middle-life and non-statutory participants viewed the place as generally in good condition but in need of constant upkeep. They gave a description of the town's physical environment as 'not outstanding in character but generally well maintained with a good mix of residential property'. The middle-lifers who gave a constructive interpretation of the physical environment did so in a way that did not run the town down and stressed what they saw as the positive aspects of what the town had to offer:

Quite tired, actually ... but it was quite quiet... Bexhill is actually quite small... it's a good centre... the scale of the place suited us very well. It still feels quite tired in terms of its state of repair... it's not that they're run down necessarily, it's just that every winter takes its toll on the 
buildings, the paintwork, the window frames and so on. So yeah, that tiredness is not necessarily years of neglect, it's just a hostile natural environment (Ref 116a)

If you go along the seafront the upkeep of a lot of the flats etc. is quite good really, because, I mean, in a seaside community it needs painting every year if you're on the front, and a lot of that is done, a lot of you know, maintenance is all part of living here (Ref 120c)

I think what we liked was it wasn't a massive housing estate where everything was the same. Yes, you've got one or two roads where there's quite a lot of bungalows, but you've also got roads, a bit like this one, where every house is slightly different and you've got a mixture of properties... in the town centre you've got some super buildings, like the Pavilion, and you've got other things around as well (Ref 114b)

Those statutory and non-statutory agency representatives that also gave a more upbeat observation of the town's physical condition did so in a similar way to the middle-lifers, in that they stated that the town was generally wellpresented:

Overall well-maintained. Not outstanding in the sense that with the exception of the De La Warr Pavilion you don't have individual buildings of great merit, but you do have an attractive town, an historic town centre and the residential areas are generally of a decent standard compared to many towns of its size (Ref 203)

I think it is quite an attractive town centre, and notwithstanding the sort of lack of new investment and development it still, you know, is quite attractive visually to look at, I think (Ref 303)

This particular non-statutory agent, who had lived and worked (on and off) in the town for a quarter of a decade, commented particularly highly on the condition and range of housing stock available in the town and on the retirement residential offer: 
I think in residential terms the built environment is very good; there is a very good range of housing. I fully expect to spend my retirement here and see just the full range of property that is available. I think the housing stock is excellent (Ref 306)

However, there were a small minority of middle-lifers who were especially critical of the town's physical condition, describing it as if it was in a very poor state:

I think the town is very disappointing, it's very tatty... I am pretty ashamed of it, actually; I think it's pretty rough and ready... it just needs a lot of money thrown at it - the whole place (Ref 111d)

The town, at the moment, does seem to be degenerating rather than regenerating (Ref 102b)

But this picture of the physical environment being derelict and in poor condition was not a commonly held view. Instead, where the town was depicted less positively by participants, it was mostly described as being 'tired' and 'in need of a lick of paint' as opposed to it being in need of 'urgent attention'. This picture of 'general upkeep' was illustrated by middle-lifers, who frequently spoke about the town in a compassionate way:

I'm quite disappointed at the tiredness of it now, but I do feel that it probably is a sign of the times rather than a reflection on Bexhill. It's tired, and that's how Bexhill is getting now, I think. It's becoming a bit tired (Ref 101c)

It's a little tired. It's a nice place to live, and I don't want to live anywhere else at the moment, but it's a little tired and it seems to need some money injected into it, I think, and the town centre needs giving a bit of a lift, really (Ref 110c) 
This view of the town's fading condition was also expressed by some nonstatutory agencies and statutory agency representatives:

Well, I think the town centre itself - there's part of it that could really do with an upgrade and an uplift... I think the built environment in Bexhill is quite poor (Ref 307)

So it does need, to put it bluntly, some tarting up, but without altering - I wouldn't want to see the quality of the town changing (Ref 206)

However, importantly, it was not the condition of the physical environment that the participants mostly remarked upon but how the town had changed in its purpose and the way it presented itself through the use of its buildings, such as retirement homes and services. The built and urban setting had altered from a town that provided up-market Edwardian tourism resort facilities and educational establishments to a place where new and existing building uses were associated with a residential retirement seaside town. These comments were particularly evident amongst the statutory and non-statutory agencies:

[It's] a residential town by the sea as distinct from a tourist resort... something that has evolved from some other primary purpose [tourism] (Ref 202)

It comes across as a retirement town... and a resort that hasn't got much to offer. It just tells me it's an Edwardian town that's had add-ons over the years... just, you know, suburban residential, really. I would, if I was coming into the town, I would I think what a shame (Ref 310)

The narratives of the statutory and non-statutory agencies referenced that the change in the physical environment away from tourism and educational facilities to retirement residential had given the town a new purpose as a seaside retirement destination. This was encapsulated in this comment from a nonstatutory representative: 
Well, obviously we are talking about some history that I wasn't around to witness, but what I do witness are buildings that, some of which have been subject to conversion, adaption or even new build, that I think were signing up to what was thought to be a regeneration initiation called retirement (Ref 301)

Another remarked on the changing offer in the physical environment away from tourism and educational facilities and towards retirement residential that reflected meeting the needs of the migration of the retired:

In the last decades I would say probably the development of quite a lot of bungalow estates on the periphery must have reinforced that... in terms of the type of housing that is being built, because that's essentially being driven by migration... we have seen very substantial expansion on the residential side... you have seen more and more developments aimed at old people, bungalows, increasing number of retirement homes, care homes which have sprung up because of the need there (Ref 203)

Many of the statutory and non-statutory agencies noted that the town's physical environment had changed after the Second World War. This was commented upon as the loss of tourism and private schools and a reduction in visitors and younger people in education, being replaced by those approaching retirement or retired and seeking residency by the seaside. And this change in the physical environment, as stated by this senior statutory agent representative, has changed the town's economic purpose:

I have been here a long time [55 years], and when we first came to Bexhill, Bexhill was a thriving seaside town... Bexhill had a number of hotels then that attracted not just the day or weekend visitors, it attracted the long stay visitors. At that time - and I'm going back 40+ years - we had... private schools, which meant there was wealth in the town because the children at those schools came from wealthy families and the schools bought a lot of money into the town centre. And Bexhill really did thrive, but... I have always taken the view that Bexhill started to 
decline with the closing of the private schools, without any shadow of a doubt, that took away a huge amount of wealth from the town (Ref 206)

Furthermore, it was the statutory agency representatives that seemed most critical of these alterations that had been allowed to happen, as presented further by another senior statutory agent:

You've got the Edwardian buildings that obviously typify the town centre, but you've also got some poorly built and poorly designed 60s \& 70s buildings. I mean, one such example clearly is in the town centre itself with the Sainsbury's structure there, but that's not in isolation (Ref 201)

This alteration in the physical environment to residential retirement, and the migration of the retired into the town, has, according to many of the participants, altered the purpose and character of the town, which is now perceived as a quiet seaside retirement place rather than the upmarket resort destination as used to be the case. This change in the use of the buildings has transformed not only the physical environment but also the social ecology of the town through migration. This is supported by the literature review, in which authors such as Florida (2008), Russell and Faulkner (2004), Champion (2005), Thomas and Dorling (2007) assert that people can shape the personality of places, whether that be through migrants in search of work, cheap housing or, in this case, for retirement purposes. This change in the physical environment in Bexhill-on-Sea, and how it may have contributed to the town's economic purpose and decline by attracting a particular cohort of retirees, is considered in the next section.

\subsection{Perceptions of economic decline and performance}

This section focuses on the economic performance of Bexhill-on-Sea and is divided into three sub-units: firstly, the participants' perceptions of the town; secondly, employment opportunities and associated constraints and thirdly, their perceptions of the localised economy. 


\subsubsection{The perceptions of Bexhill-on-Sea}

The change away from an up-market, vibrant tourism resort infrastructure towards retirement residential development was acknowledged by a number of participants. They described how the vibrancy of the town had been lost and how it was now perceived as a quiet backwater with a feel of withdrawal and tranquillity - and how this attracted retirement living. This was expanded upon by a statutory agent representative, who discussed how this perception of the town attracted those who were perhaps searching for this particular environment:

It's about its tranquillity, that its pace and its sort of timeless, timelessness, so by definition the people who are attracted to that aren't people who are attracted to dynamic, exciting, edgy places (Ref 201)

This impression of the town's environment was, for many middle-life migrants, an attractor in their migration decision, an element which is explored in more detail in the following chapter. However, this perception of the physical environment as a quiet, tranquil place that supports an ageing population not seeking vibrant economic destinations was valued by the majority of those middle-lifers who had migrated to the town, even when they were surprised at the number of elderly there were living in Bexhill-on-Sea:

The only thing I am surprised at is just how much older it is than me, but that wouldn't have influenced my decision either way, I think, had I known that before coming here. Because I think that's what helps make it a quiet place (Ref 106a)

As identified in the literature review, Bexhill-on-Sea has a disproportionate number of retired people living in the town, which in turn has attracted the label 'Costa Geriatrica'. It should be noted that the interpretation of this label was perceived and valued differently by the participants. The middle-lifers and many non-statutory agencies did not deem this label offensive but almost amusing, as if the label strengthened the feeling that the seaside town environment is nonvibrant, lacks economic purpose and is tranquil. They were not uneasy about 
referring to the town in this way, did not consider it a derogatory term and accepted this was the image for the town and valued this label:

We were initially led to believe that it's a bit of a Costa del Geriatric... we haven't got a McDonalds, we haven't got all the other fast food restaurants and we haven't got any clubs, discos and that sort of stuff in the town centre of Bexhill... I liked the idea and it sticks with me that you've got the character left in Bexhill that hasn't been taken away by the hierarchy of different, different types of generating effort (Ref 121a)

There's a certain value, and I think that might be to do with the age bracket as well because it is known that, basically, you retire to Bexhill - I suppose it's got 'one way out', that's what I get told. I don't know whether that's true - do you know what I mean? [laugh] (Ref 104a)

Yet, conversely, this interpretation was presented by the more senior officials and politicians representing the statutory agencies, and to a much lesser extent by the non-statutory agencies, as being hugely problematical, as they regarded this description and perception of the town to be negative and harmful. As this key agent suggests:

Bexhill by its nature is seen as fairly sleepy, unadventurous, unexciting...So at a very fundamental level that's quite a barrier to overcome, because it does come down to a certain extent to image. I think there becomes a point in which you have got to accept that it's the way of things, there are going to be vibrant, dynamic places and there are going to be quieter places, and that people tend to gravitate depending on what their aspirations are (Ref 202)

The change in the physical environment from a tourist resort to a retirement residential area, and alterations in the nature of the people migrating to the town and associated labels attached, had, according to a number of participants, contributed to the slow socio-economic decline in the town. It was presented throughout the participant narratives that these changes in the physical 
environment and migration patterns had altered the employment characteristics in the town.

\subsubsection{Migration and employment}

A number of the participants commented that changes in the physical environment, to support the migration of the retired, afforded fewer opportunities for people to migrate for work purposes:

It sort of tells you that it's sort of built around people that are retired down here more than people who come here looking for a job, or something like that (Ref 115b)

I think there's a limited opportunity, which means that, you know, unless you have actually got a job here, would you move here to look for a job? Probably not if you are still working, but once you come towards the end of your working career, I can see a lot of people why they would enjoy living here (Ref 304)

The recognition that employment and job opportunities within the town were restricted came through as an observation of all the participants. Those middlelifers who were looking for work (which were few) described prospects as lacking and that suitable employment was proving difficult to find. The key agents also presented a picture that work was limited, but they also added that the workforce in the town itself was a constraining factor for attracting employers. The first comment is from a middle-lifer, followed by a non-statutory agent and then a statutory agency representative:

I'm looking for work at the moment... it's very bad for work, yes... there isn't much work at all, it's very bad (Ref 115b)

I think the economic performance and Bexhill are not two words or phrases that readily, that one would associate with each other, because the considerable proportion of the town's population is economically inactive, in that they have retired to the town, and though of course they 
spend money on local services, they don't have the same economic investment in the town (Ref 306)

Without the vitality of the employment within the town I think there is a danger that the town will become poorer and that the actual fabric won't be maintained as well and therefore it won't offer the sort of attractiveness that people have come to the town for. The narrow business base, the lack of entrepreneurs within the town and the relatively low level of skills, qualifications of the workforce who are here... I think the fact is that it is perceived as a retirement town and not something that is particularly attractive to or perhaps even welcoming to business (Ref 203)

Many of the key agencies made reference to the lack of jobs and career opportunities in the town, particularly for the younger generations, of which many of the skilled and educated had left the town. They cited that the migration of the retired and those retiring into the town had created inertia and a decline in economic activity. This issue was also noted by a few middle-lifers, who were concerned about the economic welfare of the town and the younger working generation:

There aren't any jobs here. I think it's an area where there is very little work. The people that seem to work seem to work in shops and there are a lot of tradesmen, having said that, that work for themselves - builders and plumbers and such like - but for somebody such as myself, or if it had been my husband, there certainly wouldn't have been the sort of jobs we would have been looking for and at the salaries that we've been used to. Where the children are going to get jobs from when you see them all coming out of school mortifies me in an area like this... I think this is an area where probably most of the population are retired... but work, there is no work here (Ref 118c)

However, the statutory agencies and a few middle-lifers also suggested that the lack of provision of employment opportunities in the town not only affected the young but also other age groups. They described how they perceived that the 
nature of retirement is changing and that working up to and into retirement was becoming increasingly important. The statutory agencies were particularly aware of this emerging development, also supported by the literature review, in that the distinction between work and retirement was becoming less clear and far more complex:

I think a lot of people will be forced to be economically active for longer, so people will be looking for places that offer them opportunities, not just because they want to be active, but actually they will have to be active... People will have to work for longer, or even if they are drawing on pensions they will still be looking for some form of employment or some form of income to top it up, so I think job opportunities and economic opportunities will be as important to the retired generations - or people we think of as the retirement generations now - as it is to people in their 20s \& 30s (Ref 202)

I think we have got to accept that people are going to be working longer, so if they are coming here in their sort of typically, say, 55 years old, the odds are they are going to want, or need, to work as well, but they may at that age be bringing skills with them as well as experience, which in itself supports economic growth, so I don't see it necessarily as a negative or indeed taking jobs away from the younger people but part of the actual economic answer (Ref 203)

While there was some evidence in the middle-life narratives of a changing approach to income generation and employment, they were few and far between. This middle-lifer described their income strategy, similar to the approaches described in the literature review by Hewlett et al., (2009) and Cetron and Davies (2010), as being part-time, self-employed and serving the local market using their lifetime career skills:

At the moment I am retired (nurse), but I'm a self-employed health and social care trainer, so l'm going into nursing homes and other care organisations and giving training. I've taken my state pension, I've got my NHS pension and a little top up we did, so we've got enough to 
survive, so anything else is just pocket money, so there's no pressure (Ref 113b)

However, the lack of employment opportunities in the town was not an overwhelming concern for many of the middle-life participants, who were not troubled about the fact that there were limited work prospects, as they were not looking for employment and therefore this was not a factor that featured in their migration decision. Some middle-lifers even quoted that the lack of employment in the town was welcomed, as too much employment was perceived to cause the town to lose its quaintness. The town was perceived by the middle-lifers to be predominantly for retired people, and whilst retirement may in itself create low-paid employment, the lack of jobs and career opportunities was considered by a number as not being an issue for them or the town. For many, they did not perceive or believe the council's statements that the town was in slow decline. In some middle-lifers' outlooks they perceived that there was not a need for extensive employment and that the lack of employment helped 'keep a lid on the vibrancy of the town' and helped create its character by keeping it quiet and tranquil:

It's not overly commercialised... it would lose its quaintness if we go too far the other way. So it might not be what we all moved here for then (Ref 104a)

It has such a large elderly population and... it shouldn't need the level of jobs, perhaps, that is required elsewhere. Obviously the elderly require support, and that in itself will provide jobs, although they tend to be quite low-paid. The councils are always telling us how deprived both Bexhill and Hastings are, but I am not quite sure what their arguments are for that, really, to be honest (Ref 106a)

The key agency representatives described the town as having evolved from a healthy tourism and education economy to a retirement town that they described as being the result of uncontrollable exogenous forces and a change in the physical environment, which in turn led to loss of employment and a weaker economy. This was, according to their comments, due to those who 
were employed in the town being on low pay and the retired, whose incomes were fixed. Therefore, they depicted the town as having declined in economic performance and that it had become a local economy trading at a subsistence level. This supports the literature findings of Fothergill (2007) and Beatty et al., (2008) and where retail consultants Hearn $(2008,2010)$ identified the local economy as serving a local market but with decreasing footfall and spend, which the narratives of the statutory agencies questioned whether this local economy was sustainable in the longer term.

\subsubsection{The local economy}

When key agency representatives were asked about their perception of the economic performance of Bexhill-on-Sea, many related this to the high street rather than tourism, industry or commerce and described it as serving a local market. They saw retail as the dominant economic activity within the town, and while references were made to the care industry, service industries and public sector employment, these were mentioned less frequently. The statutory agency representatives described the performance of the economy in terms of a local market, with businesses apparently content to trade at a subsistence level with no great aspiration or creativity to excel or expand:

The economy is largely localised, in other words the vast majority of economic activities serve local markets rather than national or international markets... we don't have many large employers, predominately small businesses employing people, predominately from within a 10-mile radius or thereabouts. So it's quite a localised economy in many respects... we are predominately a small business economy... they know their local market, they don't necessarily have the experience or knowledge or the understanding or indeed the aspirations to look beyond that to say, 'Okay, how can I drive my business forward?' (Ref 202)

A culture within the town centre of a subsistence economy, where we've got retailers who have been there for years and years and years, most of whom are very happy just eking out a living and they would not appear 
really to have any great aspirations for either their own personal progressions or indeed following on from that the town (Ref 206)

The statutory agency representatives also made reference to how the business operations reflected the environment and how they were fairly conservative in their outlook and that they were not dynamic or creative people or businesses. This lack of commercial creativity and innovation, according to the statutory key agents, constrained the economy and had partly contributed to the economic decline of the town. They described how there was this limited aspiration and entrepreneurialism within the business community:

You're not going to get, it's not going to be the sort of place where, which is a draw for risk-takers, exciting, ambitious entrepreneurs, because they are actually going to be drawn somewhere else where they see the pickings as being rather better... the Richard Branson's of this world aren't going to come to Bexhill looking for opportunities (Ref 202)

As one statutory agency representative observed, they felt Bexhill businesses were operating at a subsistence level and relied predominately on the retirement market to serve their business. They believed there was a matter of self-interest by businesses in not disrupting the market, which may otherwise threaten their existence:

The Chamber of Commerce has a vested interest in not upsetting its main customer base, because it is largely local businesses... a community is either a market provider or a market maker, and Bexhill is largely a market provider, and that means its residents provide the market but they don't attract additionally beyond their purchasing power of significance (Ref 205)

These views from the statutory agency representatives were corroborated by some non-statutory key agents who traded in the town and accepted this localised economy. However, the traders did not present a belief that there was a problem with the local economy; rather, they viewed the town in a similar way 
to the middle-lifers, in that the local environment and the people matched their business aspirations:

My opinion of Bexhill has always been that it's somewhat unique. It is almost in a time warp, but I don't mean that in a disparaging way - I think that's one of the beauties of Bexhill and one of its benefits, which I hope people never lose sight of... When it comes to the trading side it meanders along at its own pace, because a lot of the population is of retirement age or well into retirement, and I think it's part and parcel because of the sort of make-up of the population... there's an awful lot of independent shops in the town, they all offer some sort of friendly, helpful service... it's a different culture down here. People will talk to you, and if they haven't got it, they will try and get or they will tell you where you can go to get it, and it's a nice, I find, pleasant environment (Ref 309)

Bexhill-on-Sea's economic performance was therefore presented by the key agents as limited, although, interestingly, some businesses represented that this wasn't necessarily a bad thing. The key agents described employment in the area as serving a local retirement residential market, which in turn had altered the nature of Bexhill-on-Sea's economic activity and performance. The 'blame' for fashioning this change from tourism and education towards retirement services, and the associated economic decline, was frequently placed at the local authority's door. As such, the past governance of the town is considered in the next section.

\subsection{Perceptions of the governance of Bexhill-on-Sea}

The literature review suggests that the endogenous actions and the influence of key agency governance at a local level are critical in determining the meme of the locality, which in turn produces place-specific outcomes that can adapt a resort's purpose and image. This section therefore focuses on the participants' perceptions of the interaction between the governance of the town and the processes of change. Historically, Bexhill-on-Sea Borough Council, and latterly Rother District Council (since local government reorganisation in 1974), was politically Conservative and had prided itself on local taxation being kept to a 
minimum. This was promoted in the Conservative's manifestos and continues to be the case to this day (Rother Conservatives, 2011). Those non-statutory agents, who had been working in the town for many decades, stated that they felt the council's conduct and decision-making had contributed to the decline in the socio-economic health of the town by suppressing change and regeneration:

I think a lot of blockages have been local councillors, who have been quite insular... and their own needs are not necessarily about the wider needs of the Bexhill population (Ref 307)

The council at the time - and I'm going back to the time of, it was always a Conservative Council - but the Council at the time were people who had kind of moved in to the area... they bought houses in Cooden and they lived in that area, and their idea was that we've got or we've arrived in the nice Edwardian seaside town - that's it, let's keep it that way (Ref 305)

The demands made by the retired local electorate on elected members, particularly local Bexhill ward councillors, to keep the town the same as it was the day they arrived seems to have encouraged inertia within the local council. They stated that the council had been elected by those who had moved to the town (either standing as councillors or electing councillors to represent this view) and who engendered an attitude of keeping things as they were. This observation was put forward by many of the key agent representatives who were interviewed:

The council, as with most councils, is drawing from the local populous and is dominated by those members who have the time to do it, then it probably reflects the status quo (Ref 203)

I think the intransigence for what I would describe as inner urban change was responsive to the pressures that were there within, that came from their own residents (Ref 205) 
A non-statutory agent went on to say that the consequences of the local authority action of keeping the status quo were losing skilled, educated young people from the town and driving other businesses elsewhere, thus additionally losing entrepreneurship and creativity:

The retired people coming into the town... definitely influenced the town. They didn't want industry; they played hell about Beeching Road [industrial estate]. I mean, they said we don't want that sort of thing! And so they wanted to keep it for themselves, and then over the years because they [the council] weren't investing in the town, which caused the decline... I would say the council has a lot to answer for. In the first place they wouldn't encourage industry... because of the desire by the elderly population who were running the town to remove the young people from the town; you ended up with no skilled workers here at all, and you just couldn't find skilled people... Bexhill (councillors) didn't do anything actually to sort of come back on that, they just let it happen, but there was no workforce, really, around, purely because of the structure of the population (Ref 305)

The key agencies described how the town's governance reflected retired people's desire to keep things as they are, and that 'the town has been abandoned to the elderly'. This observation, by a non-statutory agent, shows how the elected members constrained regeneration in the town:

There's this tranche of middle-aged and younger people who just don't get involved, and then the town is almost 'abandoned to the old', and I think that because of that the council is restrained by the councillors who are in power and the group that have put them there call the shots, so I think that there's this total raft of pressure on the council not to do anything radical - and something radical is needed to regenerate Bexhill (Ref 306)

As stated by this senior statutory agent, the local authority over past decades had not seen it as their role to promote economic regeneration or facilitate and support businesses within the community. Rather, retired people migrated to 
the town, and so the council may have seen their actions of restricting development and change as supporting their electorate. There were noticeable contrasting views expressed in the narratives of the key agents about the local authority's actions. The statutory agencies were critical of the Council's low local taxation policies and the unresponsive approach to business, in that this aspect of local governance decision-making had, in their view, unintended consequences that had contributed to the causes of the socio-economic problems, but they also recognised that by having low local taxation policies that they appealed to their elderly electorate:

I don't think historically there's been the drive [by the council] to actually change the town, and I think that's significant (Ref 201)

The fundamental Conservative policy was to keep the local taxation down, and they prided themselves that they have always had the lowest taxation in the county... if you go back 20-30 years, councils never really saw themselves as having a role in regeneration, and perhaps never saw themselves as having a role in preventing the declines (Ref 206)

Those non-statutory agencies that criticised the local authority for not doing anything did so by blaming those elected members that did not promote change. The councillors supported those who elected them, who generally did not want change, as this non-statutory agent went on to describe:

The obstruction that there has been to regeneration, I think, I can only infer that the council has been part of that, in that the council has not been able to drive through regeneration against the interest groups that are opposed to any of the developments that have been proposed over the last number of years... but in twenty odd years I've just heard that so many times, and it just never seems to happen. I can't think who else there is to blame other than the council, but primarily it's councillors rather than anyone else (Ref 306)

The research has evidenced that the change in Bexhill-on-Sea's physical environment, from a fashionable tourist resort to a seaside retirement 
destination, while initially induced by meta-structural forces, has been strongly influenced by the endogenous actions of key agents. These processes of change have generated an outcome that the local authority did not see coming and which was unplanned, whereby new voters do not want to see change. The town has also been noticeably fashioned by the endogenous actions of the local authority, which supports Agarwal $(1999,2002,2007)$ and Agarwal and Shaw's (2007) arguments that the internal actions of local agents in a resort can determine the trajectory of the location's TALC. The case study of Bexhill-onSea has shown that the local taxation policies of the local authority have been blamed partly for the decline. However, what also comes through the findings is the behaviour of the local planning authority and their responsive actions to the demands of developers. These planning decisions over decades have transformed the town's physical environment and modified the demographic and character of those living and visiting the town.

\subsubsection{Governance and urban planning}

Participants representing the statutory and many of the non-statutory agencies presented a view that the town, over the past five to six decades, has evolved in a manner that supports retirement migration. As stated by statutory agency representatives, the shaping of the town has been partly through internal planning development control decisions, responding to exogenous market forces, and the endogenous conduct of the local authority. These actions by the local planning authority, and governance decisions, seem to have been a significant contributor to the direction the town has taken:

Through the late twentieth century, the nature of the town subtly changed in terms of planning approvals, which was clearly gearing up for more and more for the elderly population... it will sound very critical of the council, but I think that in the past its planning policies didn't take into account regeneration or economic development (Ref 206)

Planning functions tended to be quite reactive; they waited for proposals to come in, instead of being very proactive (Ref 205) 
It went through its big expansion in the sort of 60s \& 70s, which is when really a lot of bungalow development occurred... I think it's largely responded to the market pressures, though (Ref 203)

The key agencies identified that as employment sites, such as hotels, guesthouses and school establishments, became available for redevelopment, so the planning authority approved planning applications for housing and services that predominantly suited retirement living. The physical environment of Bexhill-on-Sea therefore appears to have become structured around this demarcation between work and retirement, where those who were retiring and not seeking or needing work migrated to the town - a move which has further discouraged employment within the town. This change in the physical environment was a recurring observation in the key agency interviews and was encapsulated by these statutory agency representatives:

You could probably trace back and look at maybe some of the planning decisions that might have been taken - there may well have been a propensity to agree to the conversion of shops and businesses into accommodation, which would have seen a contraction of the economic heart of the town (Ref 206)

In terms of the worse things is, I think, the squandered opportunities of sites being used for residential development within the town centre. I think that's probably been the worse waste of opportunity, because residential is always happening in Bexhill, and there was absolutely no need to put it within the town centre. You could have created a lot of purpose and activity with it, and that would have added to the economic vibrancy and vitality of the town centre. It would have added to there being a purpose of why people would be there during the working day. It would have added to the footfall, it would have added to the spending power, so I think they were lost and squandered opportunities (Ref 205)

These responsive actions, made by the local planning authority, and the socioeconomic and cultural outcomes that then materialised were deemed by the key agents as unplanned. Whilst there was recognition that the town's physical 
environment had changed, the outcomes of these actions seemed less clear when these planning decisions were being taken. This was noted particularly by those key agents who had been in the town for some years:

I see it in the sense [the council] has responded to opportunities, but you have got to have a strategy to be able to respond to opportunities effectively. So you have got to be clear about what you want (Ref 202)

It's the result of sometimes knee jerk reactions... that before you know you have said yes to 100 planning applications, but without seeing what the total looks like (Ref 301)

The planning policies have been largely, I think, they have been largely focused around the community as it stands... they gave limited scope or no scope for radical change, and planning policies can either be benign in their nature... the planning policies have protected the inertia within the close urban environment or the central areas (Ref 205)

With planning decisions disrupting the employment and prominence given to residential retirement, some key agencies emphasised that in line with the current economic recession, a fragile housing market, future pension shortfalls and predicted changes in retirement behaviour, the consequential impact on Bexhill-on-Sea could be considerable. As this statutory agency interviewee noted:

I think Bexhill could actually become one of the biggest casualties in any potential recession, because we can completely destroy its housing market because the replenishment of purchasers would not be available... and I think as pensions are not going to be topped up by capital as much as they were in the past, pensions are going to be a lifetime achievement because of the whole problem in the pension industry... and there is only one replacement for that - and that means that you have to go back to providing economic purpose (Ref 205) 
To reverse this slow socio-economic decline, statutory agency representatives and some non-statutory agents promoted the need for strategic leadership and planning-led urban regeneration measures that would adapt the physical environment by offering more economic purpose and so better balance the demographic make-up. The first comment, by a statutory agency representative, emphasised the need to change the physical environment to attract employment into the town, while the second is by a non-statutory agent who emphasised the need for strong leadership by the council, in order to create the 'right environmental conditions' by altering the environment:

Basically, what you do is play with the demographic nature of an area, and it requires quite significant capital investment to do that, but unless you can do that, it's quite difficult to change... if you want to attract a different demographic, then you need to offer something different... so I think you have to see it not so much in terms of the housing, but in terms of the industrial and commercial floor space allocation, but you have to recognise that the two don't necessarily work in tandem - and I think that's a challenge for all of us (Ref 204)

I think it is down to the council to create the environment that will allow private enterprise to thrive and to attract those kind of businesses into the town through planning policy... there's a need for change in the council, for the councillors to drive regeneration through (Ref 306)

However, implementing this change in planning policies, and adjusting the physical environment as part of a strategy to change the demographics and culture of the town, is, according to key agencies, extremely difficult. As one statutory representative commented:

It attracts people who like the fact that it doesn't change a lot. So it does then mean that when you do make changes it tends to be perhaps harder work, not just because everybody will be critical of changes, but even the rationale for change becomes more difficult... because actually people will say, 'Well, I came here because I like the way it is' (Ref 202) 
This resistance to change within the town has meant that recent attempts by leaders in the council to transform the environment through a number of regeneration projects have been met with confrontation and opposition. Their attempt to lead and reverse the slow socio-economic decline, as demonstrated in the secondary data, is considered in the next section.

\subsection{Perceptions of Bexhill-on-Sea's future socio-economic performance}

The previous sections outlined the views of some respondents who felt that the actions of the council to enable retirement residential may have been effective in combating the loss of tourism and education and that middle-lifers and some of the non-statutory agencies, particularly the traders, seemed reasonably content with the town as it is. However, while this approach towards retirement living may have worked well up until now, the literature review forecasted that retirement may look very different in the future (DWP, 2006, 2009b; European Policies Research Centre, 2006; ESRC, 2012). The narratives of the statutory agencies stated that protecting the status quo was no longer a sustainable option, if Bexhill-on-Sea was to avoid becoming a retirement ghetto. Their representatives described that 'to do nothing' could possibly create a distinct and divided society of 'haves' and 'have-nots' within the town. A senior figure within the council put forward concerns for the future of the town, and expressed them in a forcible manner:

I think there's a realisation that the town as it is isn't sustainable... you've got an extra 20 years of life expectancy for all those people that have chosen to retire to the town; it will create a town with clearly a larger retired population, but also again I think we need to be quite mindful of the fact that those on the other end of the spectrum, who are younger people who aren't in employment... we could actually see Bexhill as a place for people that don't go to work, who are either on benefits, or they're retired... the key danger is that really we'll have a very, very clearly defined two-tier society in Bexhill of haves and have nots, and it will be a massive gap (Ref 201) 
This concern for the future socio-economic performance of the town was also presented in some non-statutory agency renditions. They observed the town was declining, regeneration was needed and more employment and wealth was required in the town:

How I see it today is not particularly good. I think it's a town that has got a lot of problems and a town that needs sorting out really in a big way... From an employer's point of view, it never did me a lot of good, because it [the town] never had any skilled people or anybody who I could actually employ, so I think it's a town that needs a bit of regeneration... you need to do something to encourage employers into Bexhill. Because unless you've got employers here, then you're not going to bring the wealth into Bexhill, mainly because they've got the spending power - the pensioners don't have the spending power (Ref 305)

The statutory agency representatives therefore promoted the view that the town needed more skilled employment and employees, and they gave examples of regeneration projects recently undertaken by the council, in order to help prevent the declines that had been identified through the secondary data:

The migration has seen an ever-increasing elderly population... Rather than saying, you know, we don't want you here, saying we do want you here, but we actually want to broaden the demographic mix in the town and inevitably that means growth in the town... what [the Council] have done recently [regeneration projects], its exactly the sort of investment... that proactive approach in terms of you bringing forward, managing small business spaces is really good (Ref 204)

However, these comments from key statutory agents, in favour of regenerating the town and bringing in more employment, were at odds with the majority of middle-lifers and those non-statutory agencies who, in the main, served this retirement market. There were varied views when participants were asked about how they would like to see Bexhill-on-Sea looking in the future and about the regeneration of the town. Some middle-lifers emphasised that the type of people currently living in the town were generally those who do not support 
change and that the current tranquil, non-vibrant environment suited their personality. It was clear that the majority of middle-lifers did not want change, as they were content in the environment in which they lived:

Well, I'm a bit worried that the council sort of tried to make it more modern; I think the Bexhill as it is, it's sort of a quite nice place... I don't think it's got nightclubs, I don't think it necessarily needs them, because there's not, like, many people that go... because people still want to come down here to retire. It's not sort of set in stone that we remain the same, but I think it caters for the requirements of most of the people here (Ref 115b)

People are what they are, unless you get a completely different set of people living here - and you're not going to get the younger people here... I'm quite happy living in a little backwater (Ref 117d)

Council leaders, in introducing a regeneration agenda through their corporate plan (RDC, 2006b) and actions on the ground, have come under immense criticism and scrutiny from various interest groups within the town voicing their objections through the local paper (Bexhill Observer, 2004, 2005, 2010, 20042013) and public questions recorded at council meetings. This opposition to regeneration was substantiated by the majority of those middle-lifers who were interviewed, whereby they expressed hostility over the recent regeneration activity. Their narratives raised concerns over disruption to their chosen lifestyle and why there was a need for change in their lifetime. The majority of middle-lifers advocated the maintenance of the physical environment, but they did not support significant alteration or regeneration:

My biggest fear is that people will try and improve it [Bexhill] and make it worse in doing so, so it almost could be improved by not doing anything. Stop trying to improve it. Tidy it, clean it, paint it - whatever you want to do - but don't try and turn it into something it isn't. Preserve what it is, which is a nice, quaint-ish south coast seaside town. We don't want to be a Hastings, we don't want to be an Eastbourne... Bexhill is a lovely place in summary, and could be spoilt, and I just hope and pray it isn't, 
and you would spoil it by trying to do too much to it. Preserve what's here; give it a polish and a coat of paint (Ref 109c)

Well, personally I hope it is not going to change too much, because the reason people come here is because they like the old-fashioned and rather relaxed way of life (Ref 108d)

When asked the question about how they saw Bexhill-on-Sea changing, a number of middle-lifers stated in a forcible manner that they did not want to see any change in the physical environment:

I don't see why it needs to change, to be honest. I'm afraid that easily lays me open to criticism - oh, he's come here and he just wants to preserve it as it is now... but I like it as it is. That's why I came here, and I suspect that goes for quite a large proportion of the residents given that so many are elderly. I mean, change is not necessarily progress, and I think that's what needs to be borne in mind (Ref 106a)

Some of the middle-life participants became quite animated and angry, with scathing comments made regarding the council's attempts to change the environment:

The frustration is the thing - the money that has been spent on the Next Wave [seafront] development and De La Warr [Pavilion], which I can't see brought any great benefit to Bexhill. I don't know what the overall game plan is (Ref 109c)

I don't like what they've done at the seafront - I don't think it's in keeping. I think it was a dreadful amount of money for very little, and I thought it was quaint before and I liked it how it was (Ref 117d)

The research findings provided evidence of a lack of understanding of the council's actions. As noted in this chapter, and perhaps surprisingly, some of the non-statutory agencies were also opposed to regeneration, as they themselves did not appreciate the reasons why the council was making 
changes and could not see the benefit to them as a business or any evidence that change would make a difference:

What's the point of regenerating the town? It's okay as it is... because there's probably an argument for leaving things as they are, and if everyone's content with what they've got, I guess it depends on that level of risk, that level of frustration that if you do nothing, nothing changes, but, you know, is there a big clamour out there for change, or is it just a perceived requirement for change? (Ref 302

Whether participants were opposed to change or welcomed regeneration, they looked to the council to show leadership, either as an agency keeping the status quo or as a disruptor.

\subsection{Perceptions of the role of local leadership}

It was conspicuous through the narratives, but also supported through local papers and analysis of secondary data, that recent council-led regeneration programmes have divided the community and the council. Those participants supporting change acknowledged the need to influence those people opposed to regeneration. They saw the role of the council as critical in leading, influencing and informing the population as to why there is a need for change and for the town to evolve. They held a view that it is down to the council to act, albeit in a managed way, to steer the town onto a course of rejuvenation or reinvention. Summed up by this middle-lifer, they implied a Lamarckian perspective where the council leadership was the catalyst for change that influenced the 'circonstances' (Butler, 1914) of their habitat and the environmental change, in order to adapt the population and its culture and thus strengthen the economy. To ease this process, some suggested it required altering the current culture and values of the town's population, as captured by this middle-life comment:

I think the first thing you have to do is actually have to influence the population of the town. I think, really, the best people to do that are the council and councillors, to persuade people that the town needs to move 
on. I believe it does need to move on. I don't say I don't want to live here, because I do want to live here, but I do believe it ought to move on. Should be more vibrant... you've got to, I believe, convince local people that your town is only going to improve if you make change. And that's the most difficult thing to do. Particularly in an environment like this (Ref 110c)

However, there were those participants who supported recent attempts at regeneration led by the council and who recognised the necessity for a determined leadership to drive through change, alter the culture and improve socio-economic performance, even if this meant coming up against opposition. This was exemplified by the following statutory agency person:

I think strong leadership from the council... there needs to be real strength which I think, you know, to be fair, councillors have demonstrated, where you find resistance for resistance sake is just to ignore that, to move past it, don't see it as a block (Ref 206)

Statutory agency representatives perceived it to be the role of the local authority to bring about change and reverse the slow decline that had been identified through the secondary data and the literature review. This was summarised by a key statutory agent:

In so far as the business community has a voice, the voice is certainly largely negative and not an inspirational voice. There is no-one from the commercial side expressing any sort of inspiration for the local economy at all... there's very limited leadership from the business community itself. You know, it's very much driven by the public sector (Ref 202)

Those non-statutory agencies, which were in support of change, also recognised the lack of leadership within their business community, with a few comments suggesting that the previous Director of the De La Warr Pavilion (who passed away during the research period) had been the only significant 'disruptor' of the physical environment in the past decade. But apart from him, key agents felt there has been no other individual from outside the council 
driving change. Those non-statutory agencies that recognised the need for leadership and regeneration by the local authority commented:

They've [the Council] got to sell themselves as a town willing to take onboard entrepreneurs and encourage new businesses into the town... it's all very safe in the fact that it's very uniformed in what people sell and what, there's no, I don't think people have got enough foresight to have a go here... it just needs people that are thinking ahead, and it's been proven that, you know, the feller that died, from the De La Warr, he thought ahead and it paid dividends no end, when they got the Gormley exhibition down here; I mean, look at the publicity it brought into the town (Ref 310)

Its more about championing a spirit that not just drives other members and officers of the council but inspires the commercial sector, the employment sector, the education sector... a politician that's a real champion, with real ideas, that has a great team, that is prepared to say difficult things, is prepared to stand up against opposition, that is prepared to say, 'Okay, guys, we've talked and we've listen for the last five years, now here's the plan and we're going to do it. And if you don't like it...' God knows what... go somewhere else! (Ref 301)

There was also a similar claim for the need to drive future change through leadership of the council present in a few of the middle-lifers' narratives:

Well, I hope councils and councillors will have the sight, the insight, to do that [drive future change]... that's their job to some extent, rather than just collecting taxes for emptying dustbins. There is more to it than that, isn't there? The structure of a town... (Ref 105d)

My view is that it's in a kind of cul-de-sac - a place where all the elderly people live - and it isn't going anywhere, and it does need to go somewhere because if it doesn't, then we might very well end up like certain bits of St Leonards and things like that - an awful place to live. So it [the Council] needs to move on (Ref 110c) 
However, according to the statutory agencies, resistance to change within the town has dampened down creativity and innovation so that any form of regeneration has proved difficult to gather support for and implement. This lends weight to the findings as noted earlier in the thesis, whereby some, namely Florida (2005, 2007, 2008), Russell and Faulkner (2004) and Russell (2006a), argue that what influences local economic success is where the most capable and skilled people locate - and where these creative and innovative people inhabit. The in-migration of middle-lifers to Bexhill-on-Sea, where employment is not an incentive for relocation, and who display a status quo orientation and don't-rock-the-boat values, has dampened down creativity and restricted attracting and retaining 'open-to-experience' types (Thomas and Dorling, 2007). This overlay between place and personality type is discussed in greater detail in the next chapter. Those people who have come to occupy the town have created inertia, as they prefer a safe and stable environment and do not want change. This aspect was best encapsulated by this non-statutory representative, who commented on the ability of the town's people to halt regeneration:

It's the example of the hotel on the seafront that was proposed a number of years ago, and which would undoubtedly have benefited the town... but after a vitriolic campaign against it by a very small but very vocal number of people, that plan was abandoned, and I think that illustrates the problem that there is in regenerating the built environment (Ref 306)

The local authority, in attempting to show leadership, is therefore trying to grapple with the dilemma whereby, ostensibly, the town is seen as a quiet, nonvibrant 'Costa Geriatrica' place to live, attracting people who seek this type of environment and who do not welcome or want change in the environment to which they move. This is viewed by some as a positive and by others as a negative, depending on the perspective of the individual or agency. Most of the middle-lifers and non-statutory agencies perceived the 'Costa Geriatrica' label as a positive, as it meant it promoted the town as a nice, genteel place to retire. On the other hand, the statutory agencies perceived this label as harmful and contributing to the socio-economic decline of the town, and in their view it made 
Bexhill-on-Sea an unattractive place for creative and innovative people and businesses to relocate. While there have been attempts by the local authority to modify this perception, principally by adapting the physical environment and attempting to alter the balance of demographics, to date this does not appear to have had sufficient impact to have transformed the socio-economics of the town. As Florida $(2008,204)$ noted, 'people seek out places to live that reinforce and reflect aspects of who they are and who they want to become'. As a result, this perception of the town's physical environment, its social, economic and cultural capital and the images portrayed, all of which give it its personality as a tranquil, genteel seaside retirement town, is further explored in the following chapter.

\subsection{Conclusion - progress with research findings}

The findings from the analysis of the primary research reveal that Bexhill-onSea, like many other seaside resorts, is perceived to have been subjected to complex meta-structural forces that have altered the dynamics of the town. However, there was strong evidence that past endogenous actions, particularly by the local authority, have significantly contributed to the town's socioeconomic decline, thereby adding to this complexity. The narrative described how, over decades, actions related to the local authority's approach to planning have knowingly and unknowingly changed the physical environment, altering its use from employment sites to retirement residential purposes and services. Governance and low local taxation policies then reinforced this protection of the physical environment for quiet retirement living, which has altered the socioeconomic and cultural capital that has been critical in producing place-specific outcomes.

The participants perceived that the local council did not stand in the way of this change, and perhaps it even saw this as an investment that moved them away from the decline in tourism and education towards a new economy, almost and perhaps unwittingly - opting to dispense with tourism (Baum, 2006). This appears to have worked well for some of the non-statutory participants and most of the middle-lifers, in that they expressed contentment with the town in its current guise. The participants' stated that this change in the physical 
environment to retirement residential had attracted and altered human socioeconomic and cultural capital. For many middle-lifers they had come to the town to retire in a peaceful and tranquil setting where most of the basic amenities were available. They stated that the lack of employment helped keep the town tranquil and non-vibrant. It was a place that was not attractive to employers or employees, and neither did it aspire to be. It was as if the town seemed to have similar traits to its resident population of wanting to keep the place as it is, with a bit of tidying up, and so the findings gave support to Florida's (2008) observations that places, like people, have personalities.

However, the statutory agencies were now questioning the sustainability of the town and that there were voices expressing it was time for change. The research on Bexhill-on-Sea has shown that those in charge of the town need to look backwards and forwards, if they are to exert effective influence over the future of the destination and reverse the socio-economic decline. The statutory agencies, in particular, were concerned that 'to do nothing' would see a continuation in the slow socio-economic deterioration currently afflicting the town, and they were therefore introducing change through regeneration projects that adapted the environment. As Mehmood $(2010,76)$ states, 'the behaviour of these key agencies is the strongest aspect of socio-economic and cultural evolutionism that allows adaptation with the changing environment'. Therefore, the leadership role, whether driving change or maintaining the status quo' appears critical in that the town leaders may be able influence the 'meme' (Butler, 1914; Krueger \& Brazeal, 1994) by altering the physical environment.

However, the majority of middle-life migrants and some non-statutory agencies did not perceive the town to be in decline and instead gave a perspective on the town's survival, its tranquillity and their wish to leave it as it is. Even some councillors were having difficulty with the regeneration agenda, as they were accustomed to stifling change in support of their elderly electorate, and so there were mixed reactions to the physical adaptations and with these views came tensions in the town's governance.

The findings identified that the image and non-vibrancy of the destination has given the people the town they deserve - a quiet, safe, non-vibrant place and 
the label of 'Costa Geriatrica'. However, critical to this research from the statutory agency and regeneration perspective is the recognition that future retirement patterns are likely to change (Heet, 2003; Lancaster and Stillman, 2003; DWP 2009a) and that resorts need to be prepared for this, especially where there is a high preponderance of retired people in the resort. Therefore, understanding the migration motivations of this next cohort of middle-lifers, approaching or in early retirement, appears critical and is examined in depth in the next chapter. 


\section{Chapter 7}

\section{ANALYSIS - IN-MIGRATION OF MIDDLE-LIFE PEOPLE TO BEXHILL-ON-SEA}

\subsection{Introduction}

This chapter analyses middle-lifers' (50-70 years of age) migration motivations to move to the seaside town of Bexhill-on-Sea and their perceptions, values and image of the place when making their migration decisions. Examining this addresses the knowledge gaps that have been identified in which to gain an understanding of these interrelationships and interconnections between processes of change in the physical environment, key agency actions and middle-life migration.

\subsection{Participant profiles}

Twenty-one in-depth interviews with middle-lifers who had migrated to Bexhillon-Sea within the past five years were undertaken, and Table 7.1, provide an appraisal of the participant profiles in five-year age groups, identifying where they migrated from, where in the town they were residing, the associated LSOA rating for that ward and the nature of the property in which they were living.

The destinations from which the migrants had relocated reflected general migration patterns from previous studies (DTZ/Pieda, 2006; DTZ, 2010; RDC, 2010, 2011c), in that 15 (71 per cent) came from London and the southeast of England area or had spent much of their lives living in these areas. Two of the participants had relocated from overseas, where they had spent much of their working lives (South Africa and Canada) but had been born in the London and southeast region, while two came from the East Anglia region and two appeared to have no particular connection to a geographical location, having lived in many locations in the UK. The housing tenure and the ratio of private to rented accommodation also reflected general patterns from previous housing studies in Bexhill-on-Sea, where outright ownership is significantly higher than the national average due to the age profile, as the elderly and middle-life people have a 
greater tendency to own their property (RDC, 2007a, 2010). The career profiles of the middle-life participants were predominantly from public services, administration and banking, each offering a stable employment environment (Appendix 24).

\section{Participant Profiles}

\begin{tabular}{|c|c|c|c|c|}
\hline Ref & Age & Migrated From & Where residing and LSOA & Property \\
\hline $\mathbf{a}$ & $50-55$ & & & \\
\hline $103 a$ & $55(f)$ & East London (Essex) & Seafront East (011E) & Owned \\
\hline $104 a$ & $51(\mathrm{~m})$ & South London & Pebsham (008E) & Owned mortgage \\
\hline $106 a$ & $52(\mathrm{~m})$ & $\begin{array}{l}\text { Devon (South } \\
\text { London) }\end{array}$ & Sidley (007E) & Owned \\
\hline $116 a$ & $53(\mathrm{~m})$ & $\begin{array}{l}\text { Midlands (North } \\
\text { London) }\end{array}$ & Collington West (009A) & Owned mortgage \\
\hline $121 a$ & $55(\mathrm{~m})$ & South London & Bexhill Down (010E) & Owned mortgage \\
\hline b & $56-60$ & & & \\
\hline $102 b$ & 59 (f) & $\begin{array}{l}\text { Hailsham (South } \\
\text { Africa) }\end{array}$ & Old Town (008A) & Owned mortgage \\
\hline $113 b$ & 60 (f) & $\begin{array}{l}\text { Bournemouth (plus } \\
\text { other UK locations) }\end{array}$ & Town centre $(011 \mathrm{~A})$ & Owned mortgage \\
\hline $114 \mathrm{~b}$ & $58(f)$ & West London & Collington West (010A) & Owned \\
\hline $115 b$ & $59(\mathrm{~m})$ & South London & Town centre (011B) & Rented (private) \\
\hline $119 b$ & 56 (f) (Dis) & North London & Kewhurst (010D) & Rented (private) \\
\hline c & $61-65$ & & & \\
\hline $101 c$ & $65(f)$ & Essex (Ireland) & Little Common (009C) & Owned \\
\hline $109 c$ & $63(\mathrm{~m})$ & South London & Galley Hill (008B) & Owned \\
\hline $110 c$ & $63(\mathrm{~m})$ & $\begin{array}{l}\text { Eastbourne (East } \\
\text { London) }\end{array}$ & Glenleigh Park (009F) & Owned \\
\hline $112 \mathrm{c}$ & $61(\mathrm{~m})$ & Hastings & Gunters Lane (009F) & Owned \\
\hline $118 c$ & $65(f)$ & Northiam (London) & Broadoak Lane (010C) & Owned \\
\hline $120 c$ & 62 (f) (Dis) & Norfolk & Town centre (011E) & Rented (private) \\
\hline d & $66-70$ & & & \\
\hline $105 d$ & $70(\mathrm{~m})$ & South London & Cooden (009E) & Owned \\
\hline 107d & 69 (f) & London (Scotland) & Little Common (009C) & Owned \\
\hline 108d & 68 (f) & $\begin{array}{l}\text { Somerset (many } \\
\text { previous UK } \\
\text { locations) }\end{array}$ & Collington (010A) & Lease ownership \\
\hline $111 d$ & $67(\mathrm{f})$ & Home Counties & Seafront (011B) & Owned \\
\hline $117 d$ & 69 (f) & Hailsham (Canada) & Old Town (008A) & Owned \\
\hline
\end{tabular}

Table: 7.1 Participant profiles

Notes: Male (m); Female (f); Disabled (Dis) Local Super Output Areas (LSOAs) 


\subsection{Middle-life in-migration motivations}

The overwhelming motivation for moving to Bexhill-on-Sea that came from the middle-lifer participants was that they 'wanted to live by the sea':

My husband retired two years ago and he really wanted to live by the sea, so we started going around the country looking at different seaside towns and we were very keen on Whitstable until somebody mentioned Bexhill, which we hadn't seen or looked at, and when we came here we really liked it and decided that's where we would put our roots down (Ref 103a)

This feature of middle-life migrants wanting to live by the sea appeared to be a key motivation even if they had no, or little, previous connection to or knowledge of the town:

When I came to retirement age, 65, and my wife sold her business and I sold mine, we sold our big house in Walton on Thames, cashed in all the chips and Hazel, my wife, said she would like to have a house on the beach, so we started looking from pretty much Bournemouth east (Ref 105d)

This statement was common throughout the interviews, in that many of the middle-lifers had little or no knowledge of Bexhill-on-Sea prior to making their move.

\subsubsection{Anywhere seaside migration}

For many of the participants the choice of seaside town could have been anywhere along the south or southeast coast, which became a crucial point in the research findings as to why Bexhill-on-Sea was chosen as opposed to any other seaside town when selecting a location based on varied reasons associated with wanting to find a better way of life by living by the seaside: 
In London... it was much busier and so on, and it was kind of a reaction to that, I suppose (Ref 116a)

For those who had explored the southeast looking for a seaside town that met their needs and aspirations, they frequently cited they knew nothing or little about the town, other than it was a retirement town:

I only knew of it by reputation as, you know, a retirement town on the coast. I had never been here; I didn't know Sussex at all (Ref 116a)

No, until I was shown the property I hadn't heard of it [Bexhill]... an estate agent in Hastings, he was showing me this property in Hastings, and I just looked at him and I said you're absolutely a million miles away from what I want, and he said I think you need to see Bexhill... So it was completely by accident really... as soon as I got here I knew this was the place to look for somewhere (Ref 120c)

In contrast to the current understanding of seaside retirement migration, not one of the middle-lifers interviewed had formerly lived in the town and was 'returning to their roots'; thus, the notion of seaside migration presented in the literature review, based on 'return' migration (Longino Jr. and Warnes, 2005), was not evident. Also, contrary to the claims made in seaside literature studies (Shaw and Williams, 1997) that nostalgia and past association with the place informed migrants' decisions, the research findings did not support this theory, as 50 per cent of the participants had no past connection with the town and had only come across it by chance. Only one participant showed any familiarity with the town, having worked in Bexhill-on-Sea (but not lived in) for a small number of years. The others had either heard about the town through marketing and media images or had some past experience of the town, having either visited friends or relatives or as part of a day trip:

We had friends that had moved from Aylesbury to Bexhill, and so we'd visited them a few times, and I think that was the first time l'd heard of Bexhill actually, when they moved here. I wasn't really aware of it before (Ref 119b) 
This finding, that in-migrants had little or no connection with the town prior to migration, is important. This cohort would have grown up during the period of emerging cheap overseas holidays at the expense of holidaying at home in the UK (Walton, 2000, 2009), so nostalgia for UK seaside towns may not be as prevalent; as such, this research contradicts the current literature. The middlelife migrants were making destination migration choices on finding a suitable location to live by the sea based on their perceptions, and their choice of Bexhill-on-Sea was fashioned by a range of migration factors and associated migration typologies, such as counter-urbanisation and experiences gathered from elsewhere, rather than a nostalgic preference for a specific location.

\subsubsection{Counter-urbanisation and the 'seaside idyll'}

Examination of the 'perceptions' provided by participants who had undertaken brief visits to Bexhill-on-Sea, before moving into the town, suggested they were founded on the strength of the town being not too large and a friendly place where people had the time to stop and talk to one another:

It was a smaller place... I think it was the small town feel and the fact that people were friendly and we thought we could meet people here. I think that was most important to us (Ref 117d)

It was noticeable in the interviews that the size of the town, the community feel, quietness and pace of life came through as strong attributes:

We walked round to get the paper, and all these people kept saying good morning, and we thought this isn't right - people are speaking to us! (Ref 114b)

If I just want a nice, quiet, peaceful time, this is it. No crowds. Nothing, Lovely! Very relaxed pace of life (Ref 109c)

These middle-life narratives are similar to those found in migration studies, where the label 'rural Idyll' is used to describe migrant perceptions that do not 
accord with the existing rural geography and are shaped by perceptions developed whilst living in a different place (Mitchell, 2004; Van Dam, 2002). In the case of the middle-life participants, the overwhelming criterion influencing decisions was that of wanting to 'live by the seaside', though the 'rural idyll' features they were seeking included a community feel, quietness and friendliness of the location were attributes they derived from their brief visits:

... a very nice village area, and everybody talks to you and everybody knows you and you know you're part of a community (Ref 101c)

The fact that it's not spoilt by loads of amusements... a quiet place, a place where you could switch off by walking along the seafront, so that's very important to me... It just felt like a community to me. You know, I thought it's somewhere we can get to know people and, you know, settle and become part of a community (Ref 113b)

This sensation and perception of Bexhill-on-Sea as a friendly place with a community feeling even came through in the key retailers' narratives:

It feels like a town where a lot of people know a lot of other people. It seems quite close-knit; it's definitely a town with a feel of community about it... they're pleasant people to do business with, they're polite, they're friendly, you know there's less confrontation, there's less aggravation in the business, you know it just feels like a nicer place to work. I mean, I thoroughly enjoy working in Bexhill - and I've worked in many places around the southeast where that would definitely not be the case (Ref 302)

Aside from the quiet and slower pace of life attributed to Bexhill-on-Sea, there were also references by the middle-life migrants to the counter-urbanisation discourse, which involved escaping from 'unsafe', vibrant, dirtier cosmopolitan places in search of a better way of life:

There was a new family moved in, next door to us in our old house, and that's really what pushed us out, because there were a couple of teenage 
lads with the tags round their ankles, abusive to their mums - it was horrific... made us move on after 25 years of being happy where we were living and they made us make our decision to move out, and I don't ever want to be in that position again (Ref 103a)

It's clean. People seem to take pride... if you walk around anywhere the gardens look pretty reasonable... I think the cleanliness of the place goes a long way (Ref 104a)

Some of the varied typologies of middle-life retirement migration often linked to counter-urbanisation that were identified in the literature review were also evident in the research findings. These are explored in more detail below, in order to understand how they impacted on motivations to move.

\subsection{Practicalities and typologies of middle-life migration}

For those having no familiarity with the town, once they visited and experienced the destination, they concluded it was the location that best suited their requirements as a migration destination:

What we were looking for was an area that had got the sort of range of facilities we wanted in the way of doctors, vets and shops and things within walking distance, fairly easy walking distance. Possible bus, train and we actually spent four months travelling the south of England. We went from Kent through to Somerset... at the end of that period we both sat down and did a separate list and Bexhill came top of the list! (Ref 114b)

The motivational desires of middle-lifers migrating to the town looking to 'live by the sea' also seemed, in part, to be influenced by the practicalities of the town's amenities and resources. A number of the participants commented that Bexhillon-Sea offered a lifestyle of 'living by the sea' in an environment that met their basic needs, such as shopping, decent transport and support services, all of which, amongst other factors, came across as seminal factors in the selection process: 
I didn't want to live in the centre of a big conglomeration, but I wanted to be near enough to a town that there would be amenities... could I get a train up to London if I wanted to go up for the weekend and how easy was it to get down to Devon and up to Scotland? I have found it very easy, but that's because I've got the time to find it easy, but if I was timelimited it would be a burden (Ref 107d)

there is everything nearby - the doctor's, dentist - in a lot of small towns you haven't got everything on your doorstep, but here you have (Ref 103a)

Some middle-lifers gave reasons for migrating to Bexhill-on-Sea that resembled previous researchers' retirement migration typologies. However, there was only limited evidence of these, such as 'assistance' migrations (Gurran, 2008):

(My husband) had an accident, a bad accident, and we decided that we couldn't really stay in the property in Ireland... (my husband) had two sisters who lived here... which brought us to this area, rather than going back to Hornchurch, which was too busy. It was (my husbands) disability; he can't walk properly, which was why we settled here (Ref 101c)

In addition, the analysis of migration typologies presented in the literature review identified the motivations for retirement migration as falling roughly into four category models (O'Reilly, 2007a), and there was evidence of motivations that are described in these models in the research data, but none of these seemed to suggest any single model could be used to categorise the motivations of in-migrants to Bexhill-on-Sea. Firstly, motivations linked to the 'life course model', where migrants tend to seek lifestyle amenities, were mentioned in this quote:

Well, the main reason was because I was too far away from my children, one of whom lives in Portslade and my daughter lives in London, so it was much more reachable. They could reach me more easily than me 
living in the West Country, and I could get to them obviously more easily as well. That was the main reason (Ref 108d)

Secondly, motivations considered in the 'migration decision model', which includes environmental adjustment in relation to, for instance, crime levels or cost of living, were also discussed in the interviews:

There were quite a few gangs of hoodies and things, and that's basically - we wanted to get away from... we wanted a quieter life... My husband really wanted to have a sea view and I wanted a garden, so we were coming here looking for a bungalow, really - really just for a quieter, more peaceful life in our old age (Ref 103a)

Thirdly, in 'the housing disequilibrium model', economic incentives related to housing assets dominate migration decisions, and for some interviewees these were indeed a consideration:

It was very much, it was house price-driven. We weren't in a position to be able to pick and choose where we lived (Ref 112c)

Fourthly, 'the place identity model' stresses motivations linked to 'selves' and place as well as 'bodies' that are mobile. These motivations were identified succinctly by this respondent:

You know, feeling as though I live here and can actually put down roots now (Ref 113b)

However, whilst examples in the data of retirement typologies were indeed previously offered by migration researchers (Champion, 2001; Longino Jr. and Warnes, 2005; O'Reilly, 2007a), none of them was dominant, and they were often cited as supplementary factors informing the reasons for migration as opposed to being key motivational factors. Instead, the motivation for migration to the town appeared to be more akin to a choice linked to 'wanting to live by the sea' and confirming an existing lifestyle, rather than establishing a distinctly new lifestyle. In the literature, the term 'lifestyle' has been used to refer to an 
increasing number of people who take the decision to migrate based on their belief that there is a more fulfilling way of life available to them elsewhere' (Benson and O'Reilly, 2009a, 608). This account, from a middle-lifer, describes how they aspired to live by the seaside, in order to attain a more relaxing lifestyle:

There is nothing I like more in the summer than visiting the coast... and I found Bexhill and sort of fell in love with it. There was something so relaxed about it... I used to come and maybe park up here and have lunch and look at these flats and think, 'I wouldn't mind living there, one day!' (Ref109c)

It has been suggested by Benson and O'Reilly (2009a, 610) that "lifestyle migration' is a search, a project, rather than an act'. Many of the participant cases had the common thread of 'wanting to live by the sea' in a quiet, safe setting, which demonstrates that these migrations could be categorised as being part of the 'lifestyle migration' phenomenon (Benson, 2007; O'Reilly, 2007a). However, upon examining the data in detail, there were some distinctive drivers behind the migration motivations that went beyond how lifestyle migration is discussed in the existing literature. To some degree, by 'wanting to live by the sea', many respondents were seeking lifestyles that they described as 'safe', 'quiet', 'clean', 'tranquil', 'friendly' and 'old-fashioned', which were terms used on frequent occasions to describe the town. The destination chosen by the participants therefore seemed to reveal aspects about the lifestyles they aspired to lead:

I think the old-fashionedness of it, the bowling green, the little parks, the values that I suppose I was going back in time, so I quite enjoyed the feeling of that... I think that's sort of stepping back into time, as it were; yeah, I liked all the old-fashioned bits that come with it (Ref 120c)

I've lived in lots of places, and everything seems to be at, like, 200 miles per hour everywhere, whereas suddenly I come down here on a Sunday afternoon, there we are back to the families on a Sunday afternoon, having a walk along the beach (Ref 104a) 
This different, or better, way of life has specificity associated with the chosen destination that reflects apparently individual preferences and aspirations. This came through the case study findings with the terms described above and how it was reflected in the narratives, with 'genteel', 'quaint' and 'traditional' being used:

Slightly quaint, old-fashioned... much more the sort of genteel seaside resort when we first came here... it's a genteel environment where people can come and they can feel comfortable (Ref 112c)

The existing literature findings on cohorts of middle-life migrants also emphasise this search for a better way of life, but they also argue that this is often associated with consumption and that this empowers people to make migration decisions that reflect their chosen lifestyle and self-identity (Sunil et al., 2007). The interpretations by Sunil et al., (2007) and Williams and Hall (2002) that migrants searching for a better way of life is connected to consumption, was not confirmed in the narratives of middle-lifers moving to Bexhill-on-Sea. Rather, a number of the participants claimed the town provided for all their basic needs and the consumption form of culture was not a high priority regarding their decision to migrate:

The shopping is perfectly adequate for our needs - we're not great ones for retail therapy, so that's all the shops we need (Ref 116a)

Furthermore, some authors, such as Benson (2007) and O'Reilly (2007a), have identified 'lifestyle migration' as an individual carrying forward a life project that is part of the reflexive project of the self, which often involves seeking a different or adjusted lifestyle through migration. Conversely, it appeared in the case study that for many of the middle-life migrants lifestyle changes linked to consumption were not a motivator for migration, but rather they were searching for community values that reaffirmed who they are and what they already have as opposed to Benson and O'Reilly's (2009a) view that lifestyle migration is based on a reflective project involving searching for a new way of life and lifestyle. The middle-life participants were looking for a seaside destination that embodied who they were, thus reaffirming an existing lifestyle, and which 
represented the lifestyles of those older people already living in the town, such as its lack of consumption, its old-fashioned look, traditional values and churchrelated community activities:

I think that had an impact on us - it was one reason why we wanted to be somewhere where there was, you know, a church (Ref 117d)

I knew it was a fairly sort of old-fashioned sort of place, and she reckoned I was very old-fashioned and l'd like it! (Ref 108d)

Therefore, previous interpretations of a consumption culture and lifestyle migration, namely being in search of a new way of life and lifestyle, as promoted by Benson and O'Reilly (2009a), was not evident in the research findings. Rather, middle-lifers moving to Bexhill-on-Sea were bringing with them their existing lifestyle and personality to a seaside environment that they perceived as more pleasant and which would not challenge them. This is a significant finding, as the case study has provided evidence of a form of 'lifestyle' migration not fully considered before, where middle-life migrants move to places that best suit their existing personality and confirm their lifestyle and life course experiences.

\section{4 'Personality' migration}

The literature review identified the need for a better understanding of middle-life seaside retirement migration motivations linked to personality that could affect the choice of destination. As such, this research draws out some significant findings. The research evidences the importance of the clustering of personality types and that the migrants weren't moving to Bexhill-on-Sea to express themselves as never before - they were moving ostensibly because it would reflect the key aspects of their previous lives, as the environment and people of Bexhill-on-Sea are felt to be familiar, aligned with an improvement on their previous residential locations. They were not seeking a new lifestyle based on some reflective project but rather bringing with them what they perceived to be the best of their existing life into a seaside town that they felt best suited their personality. They did not want the place to change but they did want cleaner, 
safer, cheaper living. In addition, they were looking for a place that did not challenge their current lifestyle but would help them to maintain it in a pleasant place next to the seaside. Lamarck observed that the environment can present itself in such a way that species adapt to that environment - 'the people like us' syndrome (Packard, 2007). So, reinvention of one's life in a 'lifestyle search' did not appear to be a strong feature of middle-life migrants; rather, the search was for a location that best suited their 'personality' and 'reflective self', as presented by this middle-lifer:

Relaxed... and everyone was so friendly and so willing to talk to me - I thought it's going to be a comfortable place to live, I like it. And having moved in, it's just confirmed all of that as well... it felt like home within about a week of moving in (Ref 109c)

As many of the participants had little or no connection with Bexhill-on-Sea before deciding to migrate, choosing this particular seaside town above others must have been based on their interpretations and perceptions of the place. The in-depth research into middle-life in-migration identified that in the case of Bexhill-on-Sea, this cohort sought out places that best reflected their personality and that gave them the opportunity to live by the sea. Places typified by new forms of consumption and vibrant seaside towns were something these middlelifers were seeking to steer clear of:

I wanted to live somewhere in the southeast... Brighton is a bit too lively... Bexhill seemed to have... the right environment... I didn't know anybody here when I moved (Ref 106a)

The middle-life participants were looking to migrate to a particular type of seaside town that best suited their personality, life experiences and social class:

People seem a little bit more relaxed - seem to have certain values... there's a certain value, and I think that might be to do with the age bracket... basically, you retire to Bexhill... simple things like Sunday not all the shops are open, which, to me, that's a great thing because I think that's where people have lost their values a little bit (Ref 104a) 
Well, my perception is that most of them are retired and middle-class...

it's a kind of fairly quiet, well-ordered environment - and that's what I like (Ref 110c)

Sweetman (2003) suggests that individuals have limited chances to participate in events outside of those in keeping with their class dispositions and that, to help make their decisions, they have no alternative other than to accept reflexivity. Benson and O'Reilly $(2009 a, 617)$ therefore suggest that 'Lifestyle choices are, therefore, a response to the increased demands on individuals to behave reflexively' rather than a clear choice. As a result, while it may look like lifestyle migration is an event motivated by individual actions and encouraged by certain forces, such as the search for the 'seaside idyll', self-realisation and consumption, counter-urbanisation and amenities, the data collected for this study also show that there are limits to middle-lifers' choices that they are unable to avoid and which are linked to class disposition. From their accounts it became evident that their migration decisions were affiliated with their previous habitus and that reflexivity was a dominant force in their choices:

I suppose it's a sort of middle-class town, and I always looked upon myself as middle class. I found them [the people of Bexhill-on-Sea] easy to talk to and get on with, you know, lots of them had the same interests as me (Ref 115b)

The 'non-cosmopolitan' feel to the town, a stable, unchanging environment and lack of vibrancy were, for the majority of middle-lifers, a positive attribute, and these accounts were supported through words such as 'clean', 'safe', 'tranquil' and 'secure'. These words were translated into a more descriptive understanding in which their meanings sometimes changed to imply the town was liked because it was 'white', lower middle class and had the feeling of a traditional 'English' residential area, which gave them a sense of security, thereby bringing with them their previous lifestyles and their own view of the world through their reflective self: 
Not many ethnic races, it's very English really, but everyone is very pleasant. That's what struck me more than anything else, is the friendliness (Ref 105d)

Some middle-life participants categorised Bexhill through traditional social groupings by placing the town and its people in a lower middle class bracket. For many of the respondents they felt comfortable with this:

I would think it's sort of staid middle class, if you like; it's not a, it's certainly not, if you compared it, say, to Margate I would say Margate is working class, Eastbourne is certainly middle class, whereas Bexhill lower middle class... there's not the sort of things in Bexhill that will attract the upper middle class people (Ref 112c)

Their migration projects could thus be seen as an outcome whereby they have been restricted to their own habitus, which in turn shapes and determines the migration decision. This demonstrated that the middle-lifers made choices which suggested that 'personality' migration and finding a location where they would 'fit-in' are individualised actions. However, these migration decisions were informed by their previous lives and were not a change from their past; rather, they were bringing their personalities and life experiences with them, including social disposition, education, skills and socio-cultural capital. Their selection of a seaside town was therefore informed by their habitus, which would impact on their migration and the seaside destination chosen. The environment that they were attracted to and have adapted to reflects a stable, secure, quiet, non-vibrant and genteel 'English' environment and image. This environment has become reinforced by the people that occupy the town and gives it its personality and cultural meme, which mirrors the habitus of the middle-lifers interviewed, who were all white and predominantly came from a backdrop of secure employment in public services, particularly the police and nursing, with local government and banking also featuring as career histories.

Middle-life migrants chose to move to Bexhill-on-Sea because of its perceived non-cosmopolitan feel, unchanging environment and tranquillity. Furthermore, they moved to the town because of their perceptions and cultural imaginings 
prior to migration, which offered a dominant personality and label of the town as 'Costa Geriatrica' that contributed to their decision to migrate. This perception and image as a retirement seaside town was an important feature in this respect.

\subsection{Image and place identity}

As many of the middle-life participants had little or no connection with Bexhillon-Sea, it was presented to the researcher that the images and labels of the place were fashioned through a range of media, fleeting visits, impressions and comparisons to other seaside towns whilst travelling around the south and southeast coast. These images of places seem to matter more to migrants in the migration search that best suited their personality than the actual qualities that could be objectively described:

Hastings, it was just too busy, I was just on my guard all the time, I didn't feel relaxed enough there so... I suppose because I was getting older I thought the facilities would be good, because there are a lot of older people here and safer and, you know, not rushing about so much (Ref 120c)

It appears the migrants were first attracted by the town being by the seaside, but thereafter, their 'first-encounter' or 'gut-feel', and the accompanying images created from their historical experiences, structure and habitus gained from elsewhere and throughout their life course, enabled them to make a choice. It appeared that this kind of migration was motivated in part by 'the place identity' and how migrants saw themselves reflected in the place to which they were seeking to move. Their narratives suggested a tendency that they looked for places that reflected their personality and attributes. So many of the participants commented on the image and their perception of the people, the town's size and its genteel, unchanging character:

The scale of the place suited us very well. It's obviously got a very elderly population, but that's not how you would necessarily characterise it once you live here (Ref 116a) 
I certainly do fit in, because most of my neighbours come from a sort of professional and semi-professional background and most of them are retired, so yes, I do fit in - I thought I would, and I do (Ref 110c)

As mentioned earlier in the chapter there were logistical and practical reasons for migration, such as housing choice or caring, but importantly there was also an identifiable emphasis on the town's image, its personality and the environment and how these mirrored and represented their own 'personality' and their migration search. This notion came through particularly strongly in the middle-life participant narratives:

I do like the atmosphere... it's a community here... Bexhill has got some personality... I do like Bexhill (Ref 101c)

This representation of the town's image and personality as 'Costa Geriatrica' contributes to the development of both positive and negative images of the town, including, as Stevenson (2003) describes, stereotyping and stigmatising. This 'naming' of the town distinguishes it and in so doing it sets it apart and gives it a label. This has implications not only for those living in the town but also for future migrating middle-lifers as they seek out places to represent their distinctive social and historic life events and personality traits. Therefore, the image and naming of the place as a quiet, non-vibrant 'Costa Geriatrica' retirement town was something that suited many middle-lifers, and the clustering of likeminded people in a place that reflected this personality came through strongly:

I think that because they [the people of Bexhill] are retired, they are looking for a quiet life, which is really what I'm after (Ref 106a)

It's an old village that's stuck in time... it's old-fashioned and it's stayed old-fashioned... that it's Costa del Geriatric! (Ref 121a)

To better understand the middle-life perceptions of the town and how this was understood in relation to their 'personality' migration, the researcher considered 
the participants' observations on the image of Bexhill-on-Sea, both before and after their migration.

\subsubsection{Pre-migration image}

As described throughout this chapter, the perception of the town before migration was that of a quiet seaside retirement place - and this was not something that seemed to deter them. Importantly, it was the perceptions of the images and labels of the town's environment and 'make-up' of the people that helped inform the middle-lifers' migration decisions:

I suppose I basically just want to live in a place that's clean, that's attractive, a place where the shops look attractive and welcoming, the people are welcoming (Ref 117d)

I would say it's quite a friendly place, really. It's a happy area... it's a nice area to shop and walk round. People always look quite cheery when they are sitting on the front, as if they enjoy living here and they enjoy the environment (Ref 118c)

The majority of middle-lifers confirmed that this image of an unchanging, quiet place was a positive attribute, and in addition they felt they had similar traits to those living in the town, thereby wanting to keep the place as it was the day they arrived:

Quiet, not much going on. My sort of place, really! (Ref 106a)

It was quite a genteel place with not a lot going on ... that sort of quietness (Ref 111d)

So, whilst the town was, in many cases, hit upon by chance, it would have been the image and first impressions from fleeting visits against which the middle-life migrants gauged and reflected how it best suited their personality. However, upon examination of their views after moving to the town, mixed messages were given. Most middle-lifers provided positive renditions about how the town 
was everything they expected and that it met their aspirations and validated decision to migrate, while others were disappointed with the outcome of their choice.

\subsubsection{Post migration - positives}

Those who perceived the image of the town as a quiet, non-vibrant place stated that they expected it to be like this and were content with what they found once having migrated. They stated that it lacked a cosmopolitan feel and vibrancy and that it was safe and clean, describing the place as if they 'fitted in'. The 'Costa Geriatrica' image, before moving, and the habitus that they found thereafter with the elderly demographic suited their 'personality' and this image helped to present the environment as unchanging and stable as described by this middle-lifer:

I know there are young people here, but generally, when you go round the shops, it's all elderly people. That is not necessarily a bad thing, though it seems to put a lid on the environment, if you understand what I mean? It doesn't seem to be very vibrant (Ref 110c)

It was apparent that to those who felt they were a 'good fit', the image and the 'reality' reflected comments about their personality matching those of people in the town:

I've joined University of Third Age, and I go to a lot of local things and I have a lot of people here as well, so yeah, there's an awful lot of likeminded people here definitely [disabled]... Yes, it will be [my permanent home]; I don't intend moving (Ref 120c)

This also included, for those who spoke positively about their migration move, the connection with churches. The image and perception of Bexhill-on-Sea having a strong foundation of churches and associated core values was an attribute presented in a number of the middle-lifers' narratives: 
Also, there's a nice selection of churches here, because I am a regular worshipper and I sing in the choir of St Augustine's and it was something you felt you could do. People didn't give you funny looks if you said you went to church (Ref 108d)

I think probably the churches make a lot of difference... I think that a lot of the cultural activities have developed out of the church (Ref 113b)

These middle-lifers confirmed how the physical environment, existing residents and the images that transpired gave them confidence that they had migrated to a seaside town where there was a clustering of likeminded people with familiar personality traits and social dispositions. However, not all of those who had migrated to the town presented a view that they had made the right choice or 'fitted in'.

\subsubsection{Post-migration - negatives and 'personality' migration misfit}

Those who felt the town did not reflect their particular class background or personality suggested they were finding adjusting to the town difficult and that they may move again in the future:

I don't like it at all, actually - Bexhill. The actual town is very, very disappointing to us after having moved here (Ref 111d)

Some middle-lifers, having migrated to the town and experienced the environment and its people, revealed that their migration decision had not been so successful for them. Many of the differences centred on the nature of the people and the negativity towards change in the environment. Once living in the town, this feature of the place not reflecting their personality goes back to Sweetman's (2003) account of the persisting influence of social structures on the individual. Therefore, for some individuals, they found they were outside of their class disposition, and they presented through their narratives that they did not 'fit-in': 
I lived in a gated estate in Walton-on-Thames... I don't want to sound condescending, but they [residents of Bexhill] are sort of a bit simpler; I mean, there are going to be educational differences and there are going to be cultural differences... I feel that the people l've mixed with here have had more of a limited experience of life than I have, really... No [this won't be my permanent home]; I feel that Bexhill does feel a little alien (Ref 105d)

I find people surface friendly, I find there's an older element that's a bit picky and, I mean, we have got friends now, but we joined all kinds of things when we first came and we just didn't feel we fitted in (Ref 117d)

Those who were disappointed with their migration felt that they had misinterpreted the perceived image of the town, based on reasons such as the limited vibrancy of the place, the superficial friendliness of residents and complaining people. These were 'personality' attributes of residents that these middle-life migrants stated did not reflect their own personality. They stated the town was 'too old' and a better balance of community was needed and that they felt 'they didn't fit'. Having migrated, they stated they found the place unfriendly and that the people's attitudes were very negative and diametrically opposed to any sort of change or regeneration:

People complain all the time and they don't like anything - they don't like what is being done at the seafront... they don't like what's being done round the De La Warr and they don't like what's being done about the parking, but nobody seems to have any really energetic or vital ideas about what they would like or what would make a difference, or what would be good, and I just feel it's got a negative tone... They just wish that everybody would stop changing things and they are so irritable about it... so I just think the population might get more and more skewed with people whose demands will really be weighted towards what older people want, which tends to make things just a little bit stolid and a little bit too careful and safe and not very exciting (Ref 102b) 
Therefore, the image and perceptions of the town before migration, in some middle-life cases, were misinterpreted and misunderstood. These shortcomings may have been based around previous visits and the image and perception given through the connection of these brief encounters:

We found it hard to find people who were kind of likeminded, I suppose, in many ways and we, yeah, I suppose l'm used to being around people who are kind of full of fun, and I came here and, you know, joined clubs and people are so serious and they've got to do everything the same way because it's always been done, traditionally, it's always been done this way... and I suppose I found that kind of mentality hard (Ref 117d)

However, there were some middle-life migrants who felt the town may offer them something in the future, if the regeneration activity, led by the statutory agencies, could alter the image of the place by attracting a different and new cohort of people:

Whenever you talk to somebody who's lived here for ten years or more, they will run the town down as if, almost defensively, they will get the negatives in first when I have no intention of being negative about the town. I think it's a fantastic place. It has its, I mean, I have used quite a lot of negative words in my description of it, but, you know, I like this place, I want to live here, it's a great place. It feels like it's just one degree off, somehow, if it could get back to its feet and find a forward momentum, get back on to the front foot, it would really make something of itself - you know, become a 'go to' place (Ref 116a)

As discussed in Chapter 6, this regeneration agenda, through altering the physical environment, exposed diverse views and split opinions amongst participants, with some supporting the changes while others vehemently opposed them. For many of those middle-lifers who felt the town had met most, if not all, of their needs and personality migration motivations, they were opposed to the changes, as they believed they would alter the town and disrupt their chosen lifestyle. Conversely, a few middle-lifers supported the regeneration programmes. These tended to be those that had found the town 
did not offer them the opportunities they were looking for, and they were therefore supporting change. The images and perceptions middle-lifers held of Bexhill-on-Sea prior to migration were clearly an influence on their decisions, but the experience of living in the town, for some, had not matched the image they had encountered prior to migration.

\subsection{Regeneration, employment and image}

When image was discussed with the statutory agencies, they suggested that the current description of Bexhill-on-Sea as a quiet, non-vibrant, noncosmopolitan 'Costa Geriatrica' retirement town was a problem that was contributing to its declining socio-economic performance:

I think the fact that it is perceived as a retirement town and not something that is particularly attractive to, or perhaps even welcoming, to business... I have certainly come across London agents who wouldn't dream of investing down here, just because of the image of the place as something of an economic backwater. So, people's perceptions have a bearing on investment and indeed on businesses moving to the area, but it also perpetuates the sort of the past trend of migration (Ref 203)

The key agencies saw the image of Bexhill-on-Sea as detrimental to the town, as it deterred other working-age cohorts and businesses from wanting to relocate to the area:

It's sort of, the analogy we get is it's God's waiting room! And so it is an image that's perceived by other people, and it's how do you get over that image? I think probably the image is one of the biggest issues (Ref 307)

In Chapter 6, the lack of employment opportunities was presented as an issue in the narratives of all the interview participants, but they were not unified. The majority of the middle-lifers and a number of the non-statutory agencies were not concerned about the employment limitations, as they stated that the town consisted predominantly of retired people who were not looking for work and 
that this lack of employment was an attribute of the town, as it meant the ‘vibrancy' was constrained:

Why do we want Bexhill to change? I am quite happy with what l've got at the moment (Ref 107d)

With regards to the middle-lifers, twelve participants were fully retired and not seeking paid employment, although a number did carry out voluntary work. Most of the participants stated they were comfortably off with their pension and did not need to work. However, there were some who expressed that they were finding it difficult to make ends meet, though they were not experiencing extreme poverty. Appendix 24 provides an understanding of the participants' current employment position, household income and career history. Two were unemployed and were in search of work, while two were employed (commuting outside of the area), four were semi-retired, having retired from their career, and were now self-employed to supplement their pension in their related field of expertise (nursing and policing). One was self-employed and holding down three roles - a part-time university lecturer, a playwright and a tourist guide:

Now I have a portfolio of work, so l'm doing some university teaching, I'm still writing plays and I'm working as an interpreter at the National Trust at Bodiam Castle... I am both employed and self-employed (Ref 116a)

Working into retirement is recognised as a feature of this age cohort (Stone and Stubbs, 2007; Cetron and Davies, 2010), and there was evidence of this with some middle-lifers. As Florida (2010) suggests, in the new economic world people will migrate to areas where they have strong employment opportunities or connections. However, it was noted that those who had migrated to Bexhillon-Sea, who were in search of work, began to realise the environment did not offer employment opportunities of value or easy access to employment elsewhere, and they informed the researcher that they may have to move again because they were unable to secure suitable employment:

When we moved here I thought it would be our permanent home, but I am not 100 per cent sure at the moment, due to the work... I was an 
administration support officer at Havering Council... and before we moved down here I got some interviews lined up for administration work and I was naive and thought I would get one of the jobs, but I didn't. I made 160 job applications; I've had 20 interviews, and at the moment I am working as a cleaner next door, but one in the nursing home (Ref 103a)

This signifies that Bexhill-on-Sea has less flexibility and versatility to respond to current and future economic conditions, as employment opportunities have been constrained through exogenous and endogenous forces. As noted in the previous analysis chapter, the statutory agencies were concerned about the impact of the lack of employment within the town, and whilst it may continue to attract the retired and retiring, it could be a mix of those not needing to work and/or those unable to work due to ill-health or limited skills, education and work prospects. However, the statutory agency representatives recognised retirement was changing and that those 'semi-retiring' may become selfemployed, to supplement or support their pensions. These variations were reflected in the narratives of the middle-lifers:

My main profession up until 2001 was police ...got invalided out...now I am a consultant educationalist, massage therapist and also first aid trainer. So when I say consultant educationalist...it's linked back to my health and safety role within the police and my time in St. John Ambulance... I will be working into retirement and Bexhill offers that opportunity through the nature of the work I do - self-employed (Ref 121a)

Of note were the younger middle-lifers who had taken early retirement but were supplementing their pension by working as self-employed consultants in their field of expertise, such as nursing, health and safety related to past policing or administration. Whilst there was recognition that other non-rewarding work was available, such as shelf-filling in the local supermarket, self-employment in their area of capability was the common feature of those middle-lifers still working: 
For most of my working life I was finance and admin manager for a family business which was our family business in South Africa, and now I am finance officer for the children's centres in the Rother area...(my husband) has retired, but I will still work I think for six or seven years...if we can afford it (Ref 102b)

With only one middle-lifer migrating for employment reasons associated with their partner, employment was not a factor in the majority of their migration decisions. However, once they lived in the town, those seeking to continue working, other than self-employment in their chosen profession, discovered Bexhill-on-Sea had very little to offer:

I really wanted to work still, and that's been a problem. I haven't found it easy finding a job... if I found a job to suit me I would be very much at home and content, but because of the work problem that is the only thing that is really putting me off (Ref 103a)

There was disparity between those middle-life migrants who were not seeking work and those who wished to continue in employment. As evidenced in Chapter 6, the physical environment did not support employment needs but rather retirement housing, which contributed to deprivation within the town. In recent times, and in acknowledgement of this issue, the council has brought in some regeneration measures to change the physical environment and alter the image of the town, in order to provoke a more socio-economically vibrant destination and attract employers and employees, as well as to adjust the demographic composition and reposition the town as a place to live and work.

These current dynamics involved in changing the physical environment are only just beginning to be played out, and they are causing tensions within the community. The 'Costa Geriatrica' image and demographic composition of a retirement seaside town are perceived by the statutory agency representatives and some non-statutory key agencies as damaging to the town and as contributing to its overall economic decline and limited employment opportunities. The regeneration activity, led by the local authority, which might create more employment for middle-lifers, was therefore presented by the 
statutory agency representatives as critical to improving the image of the town and its socio-economic characteristics. As one statutory agency representative summarised:

Create the opportunities now for, maybe, people in their 50s who are retiring early but who are still active to come into the town, to have the opportunity to perhaps turn what has been a hobby into a small business, provide a greater offer to the residents, but also so it is known nationally. I say nationally, but that's a bit grand, but so that people recognise Bexhill as a place of opportunity so that we provide this fantastic cultural and leisure offer but also opportunities for the early retirees to actually come here and maybe start a second or third career... I am a great believer it's people that make things happen, it's people that stop things happening - it's not groups/organisations or inanimate objects, it's people (Ref 206)

Therefore, key agencies' attempts to regenerate and alter the socio-economic course and cultural meme of Bexhill-on-Sea, by changing the physical environment, adapt the nature of the demographics and create new images and labels, require strong leadership. However, the local political environment and resident opposition to change are proving challenging for the local authority's key agency that is leading the change.

\subsection{Conclusion}

Analysis of the findings from the primary research in Chapters 6 and 7 has revealed that both exogenous forces and endogenous actions have created a change in the socio-economic and cultural characteristics of Bexhill-on-Sea. Actions taken by key agencies, notably local governance planning and taxation decisions, have sought to alter the town's physical structure. However, this may not be perceived positively by some middle-life in-migrants.

The principal reason and motivation given by the middle-lifers for their migration to Bexhill-on-Sea was that they had moved in order to 'live by the sea'. For a number it could have been to any seaside town along the south or southeast 
coast, as long as it was beside the seaside. They also cited that this town happened to meet most of their practical needs, such as transportation, housing and amenities, which helped inform their migration decisions that resembled retirement migration typologies linked to counter-urbanisation and migration decision models, as presented in the literature findings; nonetheless, these were not defining factors. Indeed, the findings run contrary to the literature, which actually identifies a common narrative underlying migration typologies, namely that migration is a search for a better and different lifestyle (Benson and O'Reilly 2009a). However, following further examination of the research findings, this view of lifestyle migration did not readily describe the decisions of the middle-lifer migrants in the present study.

What transpired was that middle-life migration was not about a lifestyle change but rather about a place that reflected a previous lifestyle that did not require change. Instead, the migrants brought with them their previous lives into a seaside town that best suited their personality and offered them an improved environment. They up-rooted their lives and came to Bexhill-on-Sea based on reflexivity, habitus and lifetime experiences, making assessments of the town through brief visits, marketing images and media that resulted in them feeling the town and its people best suited their personality and seemed to be a 'good fit'. This clustering of personality types gives support to Florida's (2008) notion that 'places, like people, have personalities'. Many middle-lifers liked it that way, in that their personality and class disposition reflected that of the town's environment, its residents, cultural meme and the 'Costa Geriatrica' image. This type of 'lifestyle-affirming' migration has rarely been covered by standard migration typologies.

However, changes in the town's physical environment, from tourism and education employment to retirement residential, and the current socio-economic and cultural characteristics of the seaside town were perceived differently amongst the participants. The majority of middle-lifers had migrated to the seaside town in order to retire, and they were not looking for work. They did not perceive the lack of employment as a problem, as it was a retirement town and it 'helped keep the lid on the environment'. Moreover, they were content and saw Bexhill-on-Sea as a place where they would see out the rest of their life. 
The key agency representatives, however, offered an opposing view and stated that the town was not attractive to employers and employees, as the physical environment, demographics, images and labels attached thereto had led to slow socio-economic decline. Likewise, a few middle-lifers were disappointed in their migration decision and found the town lacked sufficient vibrancy, did not offer adequate employment opportunities and that their class, social structure and personality did not match those in the town and were making them think about migrating again.

The exogenous and endogenous factors described throughout the previous chapters have contributed to the socio-economic decline of the town and provided it with place-specific outcomes. The next chapter consolidates the findings from the primary and secondary research identified in Chapters 5, 6 and 7 and argues that as the physical environment changes, often through the actions of key agencies, so the in-migration of populations that are attracted to this changing environment alters and can influence the image, personality and cultural meme of a seaside town and in turn its socio-economic performance. 


\section{Chapter 8}

\section{SYNTHESIS OF FINDINGS AND CONCLUSION}

\subsection{Introduction}

The research aims were to examine socio-economic and cultural change and regeneration in a seaside town, by using a case study to understand the interconnections between processes of alteration in the physical (human-made built and urban) environment, key agencies that influence change and the effects of middle-lifer migration (aged 50-70 years) to a seaside town. The purpose of this chapter is to synthesise the researcher's interpretations from the secondary data analysis and the previous two analytical chapters with the issues raised by the literature review, in order to generate findings.

Bexhill-on-Sea was selected as a single-case study, because it was identified as rare, in that it has one of England's highest concentrations of middle-lifers and retired people and has changed from a traditional seaside resort, with its loss of its seaside tourism market share, to a seaside retirement town with a 'Costa Geriatrica' image (Shaw and Williams, 1997; McHugh, 2003; Argus, $2005,2009)$. Added to this is a series of declining socio-economic deprivation indices (Beatty et al., 2008; ONS 2012; ESif, 2012). The primary research approach, using narrative inquiry, however, does not strive to produce any conclusions based on certainty, but it does aim at ensuring the findings are wellgrounded, in order to add to the body of knowledge on seaside resort towns.

In drawing on the literature, it can be seen the current interpretation of resort lifecycles and spatial structures that they are both exogenously and endogenously determined (Agarwal, 1997, 1999, 2005; Cooper, 1997, 2006). The impacts that these forces have on a destination may, however, not be readily apparent or evenly distributed. Thus, the study of the structural dynamics and organisational agency, and how they interplay in Bexhill-on-Sea, seeks to add to this understanding of the resort destination lifecycle and decline. As Butler $(1980,2006 \mathrm{~b})$ and other tourism researchers seem to argue (Cooper, 1997; Agarwal, 2002; Russell and Faulkner, 2004; Coles, 2006; 
Ravenscroft and Hadjihambi, 2006), a particular characteristic of a destination's evolution is that it is influenced and yet confounded by the nature, politics, demands and experience of a place, where the place gives it substance and meaning. Whether it evolves through natural evolution and survival of the fittest, or by adaptation to its environment, is observed within the case study of Bexhill-on-Sea.

The examination of the physical environment and key agency actions within the case study provided evidence that supported the literature review findings that the nature of localities and the physical environment influence people's adaptation to that environment. In addition, the research substantiated that while initially exogenous forces began to change the structure of the resort, it was the endogenous actions of key agencies that have led to socio-economic and cultural outcomes within Bexhill-on-Sea. These forces and actions have adapted the human content within the town, thereby giving it a distinct personality.

The research also shows the importance of understanding interactions between the physical environment and individual agency, and the study of the middle-life migration motivations in selecting Bexhill-on-Sea as a destination in which to live provides a significant finding for the thesis. The geographic mobility motivations of these middle-lifers seemed to challenge the conventional migration typologies and consumptive and lifestyle search presented in the literature findings by authors such as Champion (2001), Rowles and Watkins (2001), Gurran (2008) and Benson and O'Reilly (2009a). Instead the primary research generated data which could be interpreted that the migrants sought destinations whose environments, people and image best reflected their own personality. In Bexhill-on-Sea there is a migration in-flow of middle-lifers who are seeking seaside destinations that align with their personality, where they have brought their previous habitus and life experiences into an environment that is stable, quiet, safe and unchanging and best suits their social class disposition.

The remainder of this chapter analyses the findings in the gaps in knowledge and what this means in addressing the identified research problem. The 
chapter summaries the research findings in six sections: firstly, the validation of the TALC and the Lamarckian socio-economic and cultural evolutionary perspective; secondly, the interconnection between the physical environment and key agency actions; thirdly, the role of key agencies and, in particular, governance and leadership; fourthly, the perceptions of images and labels attached to places; fifthly, the migration typologies, motivations and characteristics of middle-lifers and the personality imprinted on the town and finally, the chapter concludes with a reflection on the contribution to knowledge on seaside towns that this data has generated to address the gaps in knowledge and the research problem.

\subsection{Resort structure evolution and tourism area lifecycle}

This study of structural dynamics in Bexhill-on-Sea has revealed how relationships between the various agencies are understood and adds to the learning on resorts. Bexhill-on-Sea, like other resorts, does not exist in isolation, and therefore it is not immune to the impacts of macrostructure conditions. These exogenous forces, as presented in the literature review (Shaw and Williams, 1997; Brodie and Winter, 2007; Benedict and Houghton, 2009; Walton and Browne, 2010), were noticeably influential in the evolution of the town. The description of Bexhill-on-Sea as a resort follows a similar outcome to Butler's (1980) 'S' shape TALC curve. Bexhill-on-Sea can be observed as a resort destination whose lifecycle has taken a path of 'exploration' through the innovation of the De La Warr estates, 'development' by the private sector, in partnership with municipal authority public realm investments, 'maturity' between the First and Second World Wars, 'stagnation' that followed the Second World War and the slow 'decline' in more recent decades that has witnessed the resort becoming predominantly a seaside retirement town with declining socio-economic indicators. This evolutionary cycle was evidenced in the published literature presented in the earlier chapters and supported by the narratives of a number of the statutory and non-statutory agency representatives who had lived or worked in the town for many years and were familiar with its economy and history. The interpretations of these annotations provided verification of Butler's (1980) observed TALC and followed a Darwinian socio-economic and cultural evolutionary perspective where some 
resorts survive and others fail that portrays a 'survival of the fittest' analogy. The tourism literature seems to point to this when focusing on the wider exogenous forces (Hodgson, 2005) such as global economics on seaside resort towns.

However, work by Ravenscroft and Hadjihambi $(2006,151)$ suggests that 'Butler's TALC model may, at the very most, be a broad analogy of a rather deeper and more complex process', and this complexity is evident in the study of Bexhill-on-Sea. The TALC model emphasises structure over agency (Milne, 1998; Cooper, 1992, 2006; Agarwal, 2002, 2006) but fails to consider adequately potential interdependencies between the external forces of change that are driving resort evolution and the internal forces involving key agency and human actions. Agarwal (2006) argues that the spatial variation of resort economic performance has two fundamental and interrelated dependencies: the extent human agency has over structure and the context in which this occurs. This spatial variation was evidenced in the case study and identifies how the actions of key agencies affect the nature of change in the resort.

\subsubsection{Resort evolution - Darwinian and Lamarckian key findings}

Discussed in earlier chapters, Lamarck's theory is concerned with the nature of the environment and the conditions on which animals depend (Butler, 1914; Packard, 2007). The influence of localities and the structure of the physical environment bring about or cause species to adapt their habits (Packard, 2007), and this is evidenced in the case study. For Bexhill-on-Sea, the nature of the conditions initially changed through external forces. Thereafter, enforced by key agency internal actions the nature of the town's tourism structure altered and with that the nature of the population visiting and living in the town. Thus, in Bexhill-on-Sea, the loss of tourism facilities such as hotels, guesthouses and private schools, which have been converted or redeveloped into retirement residential services, has caused an alteration and adaptation in the nature and habits of the population. The wealthy upmarket seaside resort for the fashionable, well-heeled society (Porter, 2004) seeking a vibrant environment of motor races and Kursaal entertainment (Gray, 1994, Porter, 2004) has been

adapted to a quiet, tranquil, non-vibrant, limited economic environment for lower 
middle class seaside retirement living. The people now living in this environment seek to keep the town the way it is currently presented.

Yet, in the literature review of the TALC model, insufficient attention is paid to the actions and habits of internal agencies and how this may influence the socio-economic characteristics of the town. Agarwal's $(1997,2002)$ resort studies highlight the fact that the TALC model downplays the role of human actions. Yet, evidence within the case study of Bexhill-on-Sea reveals the importance of key agency and human actions as a significant force. The research demonstrates that at a macro-level Bexhill-on-Sea's resort structure has, up until recently, evolved in a manner that emulates Butler's (1980) TALC model and is now in the latter stages of the tourism lifecycle, moving into decline. However, and importantly, while the changes were initially induced by meta-structural forces, which have affected all English seaside resort towns, it has been endogenous actions that have led to the socio-economic and cultural outcomes for Bexhill-on-Sea that appear partly unintentional but also intentional, given the recent actions of some key agencies. The findings reveal that the local authority has been a significant contributor to the direction the town has taken and its influence on its socio-economic and cultural performance and the creation of the town's meme (an element of a culture or system of behaviour passed from one individual to another through imitation or other non-genetic means) (Dawkins, 1986).

According to Lamarck, the actions and habits of humans depend upon relations with surroundings that exist habitually (Butler, 1914). Change in the physical environment, through the alteration of building uses away from employment towards retirement functions, has altered the demographic characteristics of the people who have adapted to this new environment. In-migration of the retired to an environment that supports their needs has culminated in a change of demographics and habits that are heavily biased towards those in middle-life and older age not requiring or seeking work. The middle-lifers' narratives welcomed this 'presentation' of the physical environment and the lack of employment, describing it as 'keeping a lid on the vibrancy of the town' - and so contributing towards its tranquil and quiet setting. 
What became noticeable, certainly with regard to Bexhill-on-Sea, is how far an evolutionary analogy summarising socio-economic change in the town would relate to both Darwinian selection and Lamarckian adaptation. Aunger (2001), Laland et al., (2000) and Metcalfe (1993) support that, while Darwin's theory is probably a more accurate reflection of biological evolution, Lamarck's work resonates with socio-economic and cultural evolution. However, the case study resonates with both Darwinian and Lamarckian evolutionary ideas.

Whilst there is little evidence to suggest that Bexhill-on-Sea's statutory agencies initiated the change to retirement living, once it arrived, they sought to ensure that the resort capitalised on the presence of retirement living that has overtaken and further diminished resort activity. Therefore, the evolution of Bexhill-on-Sea appears to resonate with both Darwinian and Lamarckian theory. The way in which the resort was first initiated by the De La Warr family intentionally changing the coastal environment reflects a Lamarckian socioeconomic and cultural theory. Subsequently, its evolution, in competition with other resorts and exogenous forces, was responsive (Darwinian), and now again we see this with the recent intervention in the physical environment by the endogenous actions of the local authority (Lamarckian). Ravenscroft and Hadjihambi (2006), following Gould (1990) and Steele et al., (1998), advocate that this is defensible, whereby both Lamarckian and Darwinian evolutionary constructs may operate together, at different levels and at different times. This also aligns with some of the natural evolutionary theorists such as Butler (1914, 250), who promote that Buffon, Lamarck and Darwin seem to explain that the 'doctrine of descent with modification as the explanation of all differentiations of structure and agency'. Butler (1914), therefore, sees no reason why survival of the fittest should claim to be an especial 'means of modification' rather than any other adjunct. The case study adds to the knowledge of resorts, in that Bexhillon-Sea's structural evolution appears to operate at two levels. At the macrostructural level, the conditions are more likened to the evolutionary process of Darwin, where structures are influenced by forces outside of their control, such as global economic conditions. Conversely, at a micro-structural level, the interventions and actions of internal agencies can have either positive or negative effects, depending on the active role that they play, which is Lamarckian in character. 
Thus there are aspects of socio-economic change that reflect both Darwinian and Lamarckian evolutionary thinking and that appear to reside in resorts influenced by the impact and intervention of exogenous forces and endogenous actions. It would be incorrect for those who argue for an evolutionary approach to socio-economic and cultural change to distance themselves completely from either Darwinism or Lamarckism as this case study has shown the two can be broadly interpreted as comparable operating at different levels and at different times. Ravenscroft and Hadjihambi (2006) while accepting that the timemediated dimensions of the TALC are a useful analogy for the growth and decline of resorts argue that time is a weak and unreliable reference on which to base the model. Rather than the analogy of the life cycle they argue that development of resorts is better understood as a process of evolution and this view is supported by this case study.

This is a key finding and gives substance to both approaches to analysing evolutionary change that operate at different levels and at different times in which a Lamarckian socio-economic and cultural interpretation indicates that human agency is very influential on the local environment but where structure is affected by the socio-economic and cultural climate from both external forces and internal actions.

\subsection{Interconnections between structure and agency}

This case study-based research increases knowledge regarding seaside towns by stating that broader external forces and internal responses produce 'placespecific' outcomes. Agarwal $(1999,2002)$ and Martin (2006) suggest that resort destinations are not only passive victims of global forces but are also determined by the impact of local politics and their decision-making, which is a notion supported by the case study findings. The significance of the local arena and political pressures are evidenced in Bexhill-on-Sea, as well as the extent to which these local restructuring 'strategies' have been shaped and influenced by these endogenous actions. Therefore, in order to conform to Lamarck's construct there would need to have been some explicit process of adaptation and inheritance. In contrast to biological inheritance, where it is the genome that determines variation, the resort development process is largely externally 
initiated and mediated by the 'meme' (Dawkins, 1986, 1989). The learning function in Bexhill-on-Sea is principally related to human actors, where in the past the key agencies described how the town's governance reflected retired people's desire to keep things as they are, and that 'the town has been abandoned to the elderly'. As such, the council may have seen their past actions of restricting development and change as supporting the electorate at the expense of economic activity. This was evident in the case study where, according to the statutory agency representatives and some of the non-statutory agencies, the slow socio-economic decline in Bexhill-on-Sea has been caused extensively by the actions of past leaders and the governance of the town.

The primary research findings perceived that past planning decisions were responsive to residential developers and yet unresponsive to business needs, and the authority unknowingly acted in a way prejudicial to the town's interests, which has seen the town evolve by attracting retirement in-migration to the detriment of its previous image as an upmarket resort destination. The gradual alteration of the physical environment over the past forty to fifty years, to largely retirement residential services and amenities to support retirement living, was interpreted by the statutory agency representatives as a problem and a significant factor contributing to the slow socio-economic decline of the town.

The primary and secondary research findings also identified that over the past four to five decades, elected officials had unequivocally promoted policies of low local taxation that supported the retired local electorate and which led to limited economic investment during this period in the town. The secondary data findings furthermore identified that the majority of the elected officials themselves were retired and that the key agencies recognised that those elected onto the council were there to support their electorate, who were in the main retired and who promoted the view that they did not want change and wanted low local taxation. The past manifestos of the controlling political group evidently promoted these policies (Rother Conservatives, 2011), which did not prioritise investment and regeneration. The comments from the key agencies were that it was not in the interest of the elected officials to endorse change if they were to be re-elected by a community who did not want change, and so it 
was felt that they had acted on the basis of their own needs rather than the wider needs of the town.

According to the key agencies many of the locally elected officials came from the in-migrant cohort, and they supported their retired residents at the expense of businesses, employment and a more vibrant economy. The physical environment of Bexhill-on-Sea has therefore become structured around this demarcation between work and retirement - where retirement takes precedence. The key agency narratives described that the private schools closing, as well as the decline of tourism and other employment in the town, had contributed to a change in the physical environment towards retirement residential, which in turn led to the out-migration of the young, skilled and educated seeking work elsewhere. They were replaced by the in-migration of a retiring population on fixed incomes not seeking employment, joining those who had remained in the town and were, in the main, unskilled with limited job prospects or on incapacity benefits.

The past four to five decades have seen the alteration of a number of resorts' physical environments towards seaside retirement. This slow change has often gone unnoticed; for instance, Johnston (2006) describes it as a 'blurry transition' that has gradually introduced a new and distinct retirement population into seaside resorts. The local authority overseeing Bexhill-on-Sea over this period made the decision to allow the growth of retirement residential accommodation, in order to compensate for the loss of tourism and education. However, statutory agency representatives in Bexhill-on-Sea gave the view that the town's current socio-economic and cultural offerings were now problematic, and the descriptions they offered about the limited economic performance of the town matched the patterns revealed in the earlier secondary economic analysis and IMD data on Bexhill-on-Sea (DCLG, 2007b; Hearn, 2008, 2010; ESiF, 2012; ONS, 2012), as well as the quantitative examination of seaside resorts by Beatty et al., (2003, 2008, 2012) and Fothergill (2012) which showed continuing gradual decline in the town.

The literature review identified that future retirement looks very different, and, similar to the literature findings (Hirsch, 2003; Baker, 2005; European Policies 
Research Centre, 2006) it was acknowledged by the statutory agency representatives that in the future, working into retirement may take on greater significance and may have implications for Bexhill-on-Sea. The literature notes the 'blurring' of retirement and the need or desire to continue working into retirement (Hirsch, 2003; Heet, 2003; Cetron and Davies, 2010), but current evidence of this phenomenon was limited in this case study. Only a few of the middle-lifers interviewed displayed this characteristic, so it was argued by the statutory agency representatives that the structure of Bexhill-on-Sea and its attractiveness (or reliance) as a place that entices retirement migrants who do not seek work, or who are restricted or unable to work, in a quiet, non-vibrant setting could become problematic in the future, as suitable employment is not available for those who are working into later life because it is desirable or a necessity. The alteration to retirement living in Bexhill-on-Sea attracted inmigrants who were not in search of work and a clustering of personalities that did not wish to see change to their environment or habitat. Therefore, as the interviews with key agencies showed, any regeneration changes to the physical environment proposed by local authority leaders have been met with opposition and resistance, thereby making it more difficult to adjust declining socioeconomic conditions in the town.

While the past governance of the town had facilitated retirement migration and a quiet, tranquil destination, statutory agency representatives gave the view that the current local authority leadership wanted to drive through change. The present-day administration promoted the need to intervene and adjust the structure of the physical environment, thereby changing the demographic mix of people migrating to the town and thus also altering its socio-economic performance and cultural meme. The refurbishment of the Grade I listed De La Warr Pavilion as a centre of excellence for art and culture, the upgrade of the seafront promenade as a contemporary landscape, the investment in new commercial developments and 1,500 new homes to the north of the town had, amongst other regeneration projects, shown clear intent that the local authority was seeking to alter the structure of the town. However, interviewees indicated that these actions by the statutory key agencies were being resisted by the retired resident community, who looked to their local authority elected officials to reflect and represent their opposition to these changes and their desire to 
maintain the status quo. In the literature review, Gordon and Goodall (2000) and Martin (2006) describe how the nature of communities can determine a location. This is firmly demonstrated in this case study, as the cultural meme of the population within the town and the physical environment gives the town its substance and meaning.

The majority of the middle-lifers welcomed stable, local economic conditions and stated that they were very content with their migration choice and felt it was the town for them. They expressed through their narratives very vocal opposition to the recent regeneration initiatives led by some councillors in positions of headship and whose cabinet, the decision-making body, predominantly represented the rural parishes of Rother District (Rother District Council, 2006b). This attitude by the middle-lifers, namely of wanting to keep the physical environment as it is, also came through many of the non-statutory agencies' narratives, especially those businesses and organisations that primarily serviced the retired, such as independent retailers and care homes and those who represented them, such as the Chamber of Commerce. Those participants voicing opposition to these regeneration projects stated they did not perceive the town to be in decline but rather gave a positive perspective on the town's survival and adaptation and even exit from tourism to retirement living as a dormitory seaside town. There appears to be tension and division in the town, and the actions taken by various agencies support the earlier argument by Zimon (2000) that agency and the leadership role (Russell, 2006a) have a strong influence over changes in the physical environment - and consequently the socio-economic and cultural climate. The literature review suggests that those seaside retirement towns that seek to reverse decline may require strong leadership and a culture such that an entrepreneur is motivated to act and feel capable of and sufficiently supported to face the challenges. This supportive culture was not evident in the case study. Furthermore, the town's 'Costa Geriatrica' personality and the 'status quo' mentality, according to some interviewees, have made change and regeneration difficult to introduce.

The success or otherwise of Bexhill-on-Sea may be determined partly by the resident community. Martin (2006) describes that the consistency with which the community supports or rejects the endogenous actions of the local authority 
leadership will partly depend on the nature of the people living in the town itself. The literature review showed that Martin's analysis of the research at Hilton Head, South Carolina, evidenced that the retirees, from well-educated and successful business backgrounds, had an understanding of the importance of employment in their town, and thus the community supported the principles of 'managed tourism'. Conversely, the middle-life participants in Bexhill-on-Sea preferred stability, safety and belonging and were not in search of work, and they viewed the lack of employment in the town as a non-issue, as it helped keep the town quiet and tranquil. They were opposed to those regeneration and rejuvenation activities that they perceived would disrupt their tranquil environment and seaside living. This was especially highlighted in the rejection of the planning application for a new hotel on Bexhill's seafront (Bexhill Observer, 2004, 2005) and the campaign that followed and the lack of support from the community towards the De La Warr Pavilion and seafront regeneration projects. And so the personality and culture of the community, as evidenced by Martin (2006) and Florida (2008), will have a strong influence over political leadership and key agency actions. In some resorts, such as Bexhill-on-Sea, this may create inertia and further deprivation.

\subsubsection{Role of key agency governance and leadership}

Statutory agency representatives cited that if rejuvenation is not attempted and the decline continues, then the future of the destination in terms of its attractiveness will be bleak. However, the days of municipal capitalists (Walton, 1983; Brodie and Winter, 2007) are long gone, and some commentators have argued that the role of the local authority may need to be that of a 'revitalising entrepreneur' (Russell and Faulkner, 2004) through stimulating regeneration activity. This appears to be what the local authority leadership in Bexhill-on-Sea is attempting to do by attracting external regeneration grant monies to alter the structure of the physical environment. Baum (2006), on the other hand, argues that in some failing resorts it may well be more appropriate to exit completely from tourism rather than attempt to stay in an unsatisfactory situation. He argues that reinventing a location is often necessary, and he discusses the critical importance of the identification and application of a strategy for exiting tourism whereby this is the most appropriate step to be taken. However, Baum 
(2006) also identifies that where some destinations effectively exit tourism at this stage and become dormitory towns or centres for services, often these destinations are located in areas where economic alternatives are scarce. This appears to be the case in Bexhill-on-Sea, where other employment and economic opportunities are limited, and so there has been deterioration in the socio-economic performance, which is recognised by the statutory agencies.

The secondary research, however, identified that tourism may not need to be an either/or decision and that, if it was effectively managed, it could co-exist with other industries in the town (SeaSpace, 2003a, 2003b, 2010a, 2010b; RDC, 2006b, 2007b, 2011b, 2011d). The examples of the Elva Business Centre (RDC, 2006b, 2007b) and the new commercial development by the local 'SeaChange' Regeneration Company show the diversification that is being attempted in the area. The TALC literature discusses rejuvenation, reorientation or even exiting tourism, but there is little recognition of the coexistence of these three scenarios and the evolution of seaside resort destinations where tourism development occurs alongside other industries and regeneration strategies. From a political and strategic policy perspective, the options which these rejuvenation, exit, reinvention or co-existence stages provide may be important as destinations and their resident communities evaluate their future. What this thesis does is place the overall TALC model firmly within the context of agency, governance and leadership, and policies and strategies on decision-making, the latter which Butler (1980) stressed in his original paper.

The recent intervention in the physical environment by the council leadership has sought to reverse the impact of socio-economic decline. These developments are analogous to Lamarck's construction, with council leaders deploying a range of regeneration projects that are anything but random in their application, and they suggest that the actions of planners and officials can be understood as 'developmental interventions'. Interviewees suggested that the local governance leadership has attempted to recover the town's economy by improving the physical environment through regeneration projects. Council leaders are imposing changes to the physical environment, in order to alter and adapt human social and economic capital and the town's cultural meme, thus 
reducing deprivation. Therefore, rather than the temporal inevitability of decline, as presented in the lifecycle model, it offers a more open-ended approach that links survival to adaptation. This is the very point that Ravenscroft and Hadjihambi (2006) suggest regarding the evolutionary analogy, and as presented in their study of Ayia Napa, Cyprus. In the case study of Bexhill-onSea, the town's elected officials are seeking to develop a series of improvements to the physical environment by attracting capital (in this case lottery funds, government grants and the use of capital reserves) and thus adapting the town to changing use requirements and altering the local demographic. While planners and paid officials can act on and sometimes influence these decisions, it appears, according to the interviewees, to be the governance and leadership that are seeking to drive the direction of the town, although there is tension between those who wish to stimulate entrepreneurial activity and those who wish to maintain the status quo.

The narratives of the statutory key agency representatives promoted the need for strong leadership and entrepreneurialism, if there is to be a change in the socio-economic performance of the town. The middle-life participants, in contrast, saw the leadership role as maintaining the status quo. In this case, as Zimon (2000) recognises, elected representatives are an essential (cultural) component of the resort system, such that they, rather than the physical form, influence and guide the meme. The primary research findings placed particular emphasis on elected officials rather than paid officials as determining the future evolution and direction of the town.

If a socio-economic and cultural Lamarckian evolutionary perspective is accepted, it may be possible for key agencies to formulate policies and entrepreneurial actions to prevent resort decline. According to the interviewees, the decision by local authority leaders to rejuvenate the town represents a significant shift in thinking towards strategy and the long term. However, the capability of a destination to entice entrepreneurs from outside is often reliant on the culture and entrepreneurship that already exists within the destination (Russell and Faulkner, 2004). According to Russell (2006a), the environmental characteristic that is most conducive to entrepreneurial behaviour is turbulence. Turbulence, even mild instability, as apparent in the case study, was met with 
opposition and resistance by the town's older community, whose culture and personalities do not welcome or want change. This was evident in the primary research undertaken with the middle-lifers and the secondary data analysis of reports and documentation. Thus, tensions exist between the leadership of the council and the retired resident community and their elected ward representatives over the most appropriate strategy and course of action for Bexhill-on-Sea. The urban regeneration literature identified different strategic approaches to regeneration, either 'top down' (McCarthy, 1995, 1998; Stead and Hoppenbrouwer, 2004; Ambrose, 2010) or 'bottom-up' (Abercrombie and Warde, 2000; Dearlove and Saunders, 2000; Tallon, 2010), which different central governments have imposed and adopted, with different responses to each one in a variety of communities and localities.

According to the statutory agencies, resistance to change within the town is caused partly by the overlap between the personality of the place and the people who live and migrate there. It is, however, recognised by Cooper (2006), Agarwal $(1999,2002,2006)$ and Coles (2006) that destinations function through complex environmental, political, economic, social and cultural systems, and it is impossible to disentangle these systems during a process of rejuvenation. Hence, Russell and Faulkner (2004) and Cooper (2006) recognise that leadership and a strong political will are critical, particularly in the early stages of rejuvenation, and that a local champion is the single, most important success factor identified in the literature (Mueller and Thomas, 2000; Russell 2006a). This involves managing the political process by engaging with the community and involving constituents who often have no interest in economic regeneration, who see these actions as an intrusion on their tranquil idyll. All these complex issues were evidenced in the findings for Bexhill-onSea. However, the retired resident community, predominantly voters for local politicians, seem to carry great weight. They did not want change but instead the retention of a stable, quiet environment, and they looked to the public authority leadership to maintain this situation. This leadership quality and political will, whether for change or maintaining the status quo, appears in the case study to be the agent that could shape the structure of the town and influence the socio-economic and cultural performance and thus the meme. If political leaders want rejuvenation, then they have the ability to influence, 
providing they remain elected. This is where, as Packard (2007) described of Lamarck's work, 'there is no absolute stability only relatively stability', echoed in the words of Lampedusa $(2007,29)$ that 'If we want things to stay as they are, things will have to change'. So it appears it is through the generations that may result in either rejuvenation or further decline, depending on the direction and decision-making of the political leadership and the entrepreneurialism that they show.

If this is the case, then Lamarck's second law of heredity (that such changes are inherited and that change propagates itself) (Packard, 2007; Rensma, 2013) becomes important. Therefore, there is a critical need for improved information on the nature of resort change for communication to both elected officials and those residents resisting change in order to facilitate a better understanding of the resort functions and the influence, for better or worse, they exercise over the future socio-economic and cultural environment. This may include learning from what has happened elsewhere, but this requires strong political leadership, which can be fickle due to the nature of the very environment in which political leaders exist. Politicians can come and go, elected into office by their residents. Therefore, any change in the physical environment needs to be explained carefully and understood if they are to have a chance of succeeding in reversing the decline of the town.

\subsubsection{Structure and agency key findings}

The examination of the interplay between agency and structure revealed the impact and intervention of exogenous forces and endogenous actions that operate at different levels and at different times. At a local level the interconnections between the physical environment and key agency actions provided evidence that the nature of localities and the physical environment influences people's adaptation to that environment. This interaction at a local level and the interconnections between key agency actions, changes in the physical environment and adaptation of human social, cultural and economic capital, is crucial in the understanding of resort regeneration. The case study of Bexhill-on-Sea contributes to that understanding, in that it highlights how the local economic and social structure has been affected by external and internal 
socio-economic forces and how the place has evolved. The case study has evidenced, and gives support to Russell and Faulkner (2004), Cooper (2006) and Russell (2006a), the importance of leadership and entrepreneurialism and the need for a supportive culture, in order to create the right conditions for the town to evolve and rejuvenate. With the town's ageing community resisting change to their environment, and the lack of creative, innovative people and business headship, the interviewees claimed this role of leadership has fallen to the local authority. Nevertheless, the typology of people in Bexhill-on-Sea and its ability to 'evolve' seemed to constrain severely the work of key statutory agencies and what they perceived as having a positive influence on the future of the seaside resort town, as it is the community who elect the local administration into office. This is a contributory finding in understanding the evolution of resorts and the importance of the leadership role of the local statutory agency in influencing the meme.

The local governance leadership and their endogenous actions have been responsible for determining the dynamics of the town and its meme, where the people generate the culture, images and labels. It is this perceived image and culture of Bexhill-on-Sea that was an important influence on the middle-lifers' migration choice of destination. Hirsch (2003) suggested that some people see themselves as old, while others of the same age perceive themselves as much younger than their real age, and so the attempts by the local authority to alter the town, to present it as a more vibrant and economically successful place for younger people, was resisted by the middle-lifers and many non-statutory agencies who desired the status quo and perceived the town (and in many cases themselves) as old. With limited employment opportunities (RDC, 2006a; Beatty et al., 2003, 2008; ESiF, 2012), this may have consequences for the future of such seaside resort towns, as this inequality is expected to become more prominent over coming decades with the emergence of the 'haves' and 'have-nots' in society (Bailey and Livingstone, 2007). The research has evidenced how the town has changed and has followed the TALC curve, but nevertheless it has been given its own uniqueness by the internal actions of key agencies and local residents, which in turn have led to its socio-economic decline. The evidence in Bexhill-on-Sea revealed that such actions by elected officials has changed the physical environment, and with that the adaptation of 
those living in and visiting the resort for retirement purposes, imprinting new labels and images and attracting a particular cluster of personality types.

\subsection{Perceptions, images and labels - contributory findings}

Middle-lifers' perceptions of Bexhill-on-Sea, generated through fleeting visits and associated media images and labels attached to the place, came through as an important component in their migration decision regarding selecting a place in which to 'live by the sea'. However, this perception of Bexhill-on-Sea as a quiet, tranquil and non-vibrant place, along with the attached label of 'Costa Geriatrica', was perceived and valued differently by the research participants. The middle-lifers and many non-statutory agencies felt that Bexhill-on-Sea has already abandoned tourism and is no longer a resort; rather, it has become a gated retirement community, not in the physical sense but through the perceptions and images of the place as interpreted through signifiers in the physical environment such as retirement homes and its labelling as 'Costa Geriatrica'. The middle-lifers and many non-statutory agencies did not deem this label offensive but as almost amusing, as if it strengthened the feeling that the seaside town environment was non-vibrant and tranquil. They were not uneasy about referring to the town as 'Costa Geriatrica', did not consider it a derogatory term and accepted this was the image for the town and valued it accordingly.

This was in complete contrast with the statutory agencies and a few nonstatutory agencies, who perceived this image and the town's personality as problematic. Their perception was that the image portrayed of Bexhill-on-Sea was a contributor to declining socio-economic performance, as it did not encourage the retention of industry or attract entrepreneurial businesses and innovative or creative people. The statutory key agencies' assumption was that this labelling of Bexhill-on-Sea discouraged entrepreneurial talent from migrating to, or future entrepreneurs and innovators remaining in, the town, thereby supporting Florida (2005, 2007, 2008) and Florida and Mellander's (2007) work on the clustering of personalities. This reinforces the earlier comments of Florida (2008), whereby places, like people, have personalities from which images are created. Martin (2006), Lincolnshire Coastal Action 
Zone (2006), Margate Renewal Partnership (2008) and other such literature have shown the impacts, both positive and negative, of image on a destination. However, in contrast to the statutory agencies, the image and labelling were welcomed by middle-lifers and many non-statutory agencies, as it presented (and protected) the town as a nice, quiet place in which to come and retire. Therefore, as Butler (1914) promotes in line with Lamarck's work, those best fitted to their environment or, as Lamarck calls it, 'circonstances' are best fitted to comply with the conditions of their environment.

While the principal motivation stated by those middle-lifers who had migrated to Bexhill-on-Sea was to be able to take up a retirement life living by the seaside, the findings showed a far more complex, underlying motivation. This desire for seaside retirement within previous migration studies suggested that nostalgia for a particular resort was how retirees were drawn to a destination (Warnes and Law, 1982; Shaw and Williams, 1997; Longino and Warnes, 2005; Johnson, 2005). However, in this case study, prior knowledge or experience of the seaside town did not play a part in the choice of location; in fact, many of the middle-life participants had no past association with the town, while others stated that they had not even heard or knew of Bexhill-on-Sea until they were searching for a seaside location on the south or southeast coast. Therefore, Bexhill-on-Sea does not attract middle-life migration based on nostalgic memories as seaside historians imply (Walvin, 1978; Warnes and Law, 1982; Shaw and Williams, 1997; Watten, 2000; Walton, 2000; Longino and Warnes, 2005; Walton and Browne, 2010). The middle-lifers gathered information on and perceptions about the place through visiting friends or relatives, through brief visits travelling around the coast and drawing up a list of seaside towns that best suited their needs, from personal experiences gathered elsewhere that they imprinted on the town and from images and labels that were attached to the town through media marketing messages, such as 'Costa Geriatrica' and 'Retirement Coast' (Argos, 2005, 2009; Telegraph, 2010b). This finding is significant, as perceptions and values were central to the choice of destination, rather than nostalgic memories and any previous association with the locality. Middle-life migration to Bexhill-on-Sea was therefore based on a perceived image of the place, gut-feel and how the in-migrants would best 'fit-in'. 


\subsection{Middle-life migration to Bexhill-on-Sea - significant and key findings}

The primary research findings challenges previous retirement migration research and addressed a significant gap in knowledge. While the research uncovered characteristics of migration typologies that resembled those identified in previous migration research, association with these migration typologies were not overwhelming. For instance, there was evidence of motivations linked to 'property migration' (Rowles and Watkins, 2001), 'counterurbanisation' (Champion, 2001), 'amenity migration' (Gurran, 2008) and the influence of place-based factors similar to the rural idyll (Urry, 2002; Baker and Speakman, 2006) that could be described as a 'seaside idyll', where place and community attributes and perceptions such as tranquillity, safety, unchanging and traditional living take on similar meanings at the seaside to those identified in rural communities (Van Dam, 2002). Only partial evidence was offered where, for instance, some of the middle-lifers stated their reason for migrating was to escape rising crime from the area whence they came, some gave reasons of ease of access to family members, while for others this was an area where the property was affordable. But none of these stood out as leading reasons for selecting Bexhill-on-Sea. Initially, the motivational factors influencing in-migration appeared to be one more akin to a lifestyle migration choice of 'wanting to live by the sea' in a new environment, living out a new lifestyle. Benson and O'Reilly (2009a) attempted to encapsulate the various migration typologies, as described earlier, under the concept of 'lifestyle migration', and they suggest migrants seek a new way of life and lifestyle. However, critical to the present research was that the middle-life migrants were not seeking to adopt a new lifestyle in a seaside location.

An in-depth examination of the narratives challenges this 'lifestyle' and other retirement migration typologies and instead presents new knowledge in retirement migration motivations. Benson and O'Reilly (2009a, 2009b) suggest lifestyle migrants search for a better way of life, somewhere where they can lead a new life and that the places selected by lifestyle migrants can reveal much about the lives that they aspire to lead. Conversely, it transpired through the research that the middle-life migrants moving to Bexhill-on-Sea were not seeking to adopt a new lifestyle through migration to the seaside; rather, their 
migration motivations involved the search for an environment and community that were a reflection of who they were, rather than some search for a new lifestyle. This recognition that middle-lifers were not seeking a new lifestyle was a fine distinction, as some did feel they were leading a new lifestyle, in that they were carrying on as they were previously but in a new place by the seaside and surrounded by new people. It seemed they did not arrive in Bexhill-on-Sea to take up a new persona or lifestyle but that their decisions were informed by their lives before migration, and in this respect, they did not break from their pasts but rather brought their personalities and life experiences with them.

Their migration motivations involved a search for places by the sea that best suited who they were and their existing personality. This challenges the generalisation that baby-boomers, who were bought up in a consumer society, can be defined by 'lifestyles', 'niche markets' and 'target consumer groups' (Bauman, 1998; Hall, 1998; Blow et al., 2004). Instead, the middle-lifers seem to adhere to Bourdieu's (1984) views on how upbringing, social environment and class are influential, and that people fulfil their destiny as members of a social class. The middle-lifers' choice of Bexhill-on-Sea seems to reflect this class disposition, as in the environment and the people are familiar, and that the seaside town reflected who they were and that they seemed to be a 'good fit'. They were not searching for a new lifestyle or consumption lifestyle, as many authors prescribe, such as Giddens (1991), Zukin (1998), Williams and Hall (2002), Clarke (2003), Jayne (2006) and Mark (2007), but rather bringing their existing life and social class to the new destination that they perceived best matched their social structure and personality. Bexhill-on-Sea was selected as a place that offered them the opportunity to find a seaside environment containing similar, likeminded people and a stable, unchanging and quiet environment where they re-affirmed their existing life. They up-rooted their lives and came to the town based on reflexivity, habitus and lifetime experiences, making assessments of the town's environment and its people through brief encounters, marketing images and media labels and bringing with them their existing life to a seaside town that they perceived to best fit their personality. This 'lifestyle-affirming' and personality migration was a significant and key finding of the research. 
Researching the socio-economic and cultural roots of English seaside resort towns has strengthened the observation that places, like people, have personalities and that people are attracted to the personality of a destination that best reflects who they are. This supports the work of Florida (2008) but also strengthens the work of Bailey et al., (2006), who asserted that a place makes a difference to behaviour and so, conceptually, a destination can be considered as an influence on those living in the locality and how they act.

\subsection{1 'Lifestyle-affirming' migration and 'personality fit' within Bexhill-on-Sea}

The middle-lifers' narratives offered a view that their migration choice was inhibited by their previous habitus, in that they were looking to relocate their existing lifestyle and bring this to a new location by the seaside. Therefore, the selection of Bexhill-on-Sea, while a deliberate act about how to live, appears to be partly the result of both individual agency and structural constraints and their reflexive habitus (Hochschild, 1983; Bourdieu, 1984, 1990; Sweetman, 2003). Their migration projects could thus be seen as involving decisions that seemed to have been constrained within their own habitus, for instance their career history in stable and secure jobs. Therefore, while at first sight Sweetman's $(2003,530)$ description that 'the break-up of organised modernity has involved a shift from socialised to privatised modes of consumption', and that their migration was influenced by an individualised action, on reflection it becomes evident that there were limits to their actions and it was perceptible from their accounts that reflexivity and class disposition influenced their choice of destination, as they frequently stated that Bexhill-on-Sea felt like a place where they would fit-in. This seems to support Sweetman's $(1999,2003)$ observations of individuals in a consumption society, where in contrast 'some individuals revel in creative opportunities afforded them', while others see the consumption society 'as a risk that entails new responsibilities' and feel more at ease within the grouping of 'one's identity in a coherent lifestyle that accords with their class background and habitus' (Sweetman, 2003, 532). It is this latter point where the findings of this study support the work by Thomas and Dorling (2007) and Florida (2008) on the descriptions of personality types and their clustering effect. The middle-lifers were therefore migrating because the environment of 
Bexhill-on-Sea seemed to reflect the best aspects of their previous lives, namely that the environment and the people were familiar, which aligned with the 'agreeable and conscientious' types, as described by Thomas and Dorling (2007), that liked stable, secure and non-vibrant environments.

According to the middle-life migrants, the personality that the town of Bexhill-onSea constructs, through its physical environment, its people and images, is that of a genteel, tranquil and unchanging place with a community of likeminded, traditional British people. The accounts of the middle-life participants led the researcher to recognise that their migration decision was strongly based on the identity and personality of the town and its residents and how these reflected their own personality. The middle-lifers described their perceptions of the elderly and middle-life cohorts already living in the town as holding oldfashioned values and ideals with a good community spirit, which in part give the town its safe, quiet and non-vibrant image. The town was chosen as a final destination not as part of a lifestyle project but rather as a place that offered the opportunity to find a seaside environment with a clustering of similar people. They were not seeking to invent a new lifestyle or image; instead, Bexhill-onSea offered them a slice of 'old' and 'faded' seaside England, which gave them a sense of familiarity and security to sit comfortably alongside their previous life.

A number of the participants described the town as having a large elderly population, which suited them, as they also saw themselves as growing old, even though some of these comments were made by those in their early $50 \mathrm{~s}$. The research identified that the decision to migrate to Bexhill-on-Sea was heavily influenced by the 'personality' of the place, i.e. where the migrants wished to reflect their own personalities. Therefore, as Florida (2008) promotes, it was evident in Bexhill-on-Sea that places, like people, do indeed have personalities and that these people were happier and more fulfilled in a place that matches their own personalities. This perception of the town and its people is also partly a reflection on the nature and habitus of the interviewed middle-lifers, who were all white and predominantly came from a background in public services, particularly the police and nursing, with local government and banking also featuring as career histories. Hochschild (1983, 20-21) suggests that 'the reflexive management of one's emotions is strongly contextualised and 
tied up with particular occupational roles', which was evident in that many of the middle-lifers came from stable and secure career backgrounds and therefore were not looking for exciting, 'edgy', cosmopolitan places; in fact, just the opposite. Apart from the odd exception, they were not seeking economically vibrant places to settle, as they wanted to live out a life in a quiet seaside town with likeminded people. They were not migrating for work purposes or seeking to take up a 'second act', so to speak (Moen, 2005), and a number of them questioned the need for employment at all, as the town was predominantly full of retired people who did not require work and the lack of economic activity helped keep the town tranquil.

Having migrated to the town, those middle-life participants who stated they had had made the right choice talked about how they felt they 'fitted in', whether through the church or other community organisations, such as the University of the Third Age. It appeared that this kind of migration was motivated, in part, by 'the place identity' and how the migrants saw themselves reflected in the place they had moved to. So for the majority of the participants they commented on the town's size, its genteel character, a feeling of tradition and the perceived image of a quiet retirement place. They brought with them skills, expectations and aspirations from their lives before migration, so their migration choices remained mediated by their habitus, framed by their educational, cultural and social capital that would impact on the decision to migrate and the destinations chosen, as well as the life they then led in the destination. However, a small group of middle-lifers were disappointed with their migration choice and cited the town's lack of vibrancy and limited social and economic opportunities. It was as if their own assessment and perception of the town had been misjudged and misinterpreted when determining where they were going to migrate.

Moreover, they stated that they felt they 'did not fit-in' with the educational and social class of people and they would most likely move again.

For the majority of the middle-life migrants this self-description and perception of the town as a lower middle-class destination appeared to suit them - and they were content with this situation. The 'Costa Geriatrica' label and descriptions by the participants as a quiet backwater were, for many middlelifers, positive, as it gave them a feeling that it was a place that did not change 
much and suited their personality. Even when some middle-life participants commented that having moved to the town they had found it to have more elderly people than they expected, they frequently presented this as a positive, as it contributed to its tranquillity. They were comfortable with the town's physical environment, its people and thus its perceived character. This research in Bexhill-on-Sea has shown that these reflexive opportunities, for some people, are quite limited and they emerge from their class disposition and the habitus of the individual.

Therefore, the generalisation from the literature review that middle-lifers who have grown up in a consumer society are defined not by broad social economic demographic structures such as class, age, gender and ethnicity but are rather defined by 'target consumer groups' and 'lifestyles' (Bauman, 1998; Hall, 1998), is offered an alternative insight by this research. The case study presents a different way of conceptualising the problem in that the findings strengthened the proposition of identity with class disposition that reflected occupational roles, lifetime experiences and social upbringing that they sought to carry these with them into a new environment by the seaside with people that were generally the same. As Hetherington (1998) noted, such attempts towards 'stability and belonging' could be a response to the increasing demands for a constant and habitual reflexivity. The same is true of the adoption of particular lifestyles, which may also 'reflect an attempt to evade demands for an ongoing reflexivity and to fix, or 'anchor' the self in what can be regarded as a modernist response to the contemporary changes in the social terrain' (Sweetman, 1999, 71). The migration decision of these middle-lifers therefore supports Sweetman's (2003) arguments that despite the rise of consumption-oriented lifestyles, there is little scope for individuals to engage in actions that are outside of keeping with their class dispositions and life experiences. Habitus grants a certain freedom of movement, albeit subject to various limitations and constraints, so these middlelifers were influenced by their existing habitus in seeking migration destinations that allowed them to remain attached to their previous lifestyle. Bourdieu (1984: 175) emphasises this point by stating 'how one is predisposed to their social environment that is a product of their upbringing, and more practically one's class'. So, in a sense, habitus at least partially reproduces social structure so that individuals, to some degree, can fulfil their destiny as members of a social 
class. This 'attachment' to the safe, secure, non-vibrant and limited economic environment, and the class nature of people, supports the earlier key finding regarding personality migration. It may also provide some explanation to local authority leaders experiencing enormous opposition by elderly residents to their regeneration programme, and why some resort lifecycles behave differently to others.

The case study reveals that the town attracts those middle-lifers who have similar personalities to its residents and the town's perceived image and personality, whereby they felt they would 'fit-in' with likeminded people and its culture. In Bexhill-on-Sea there is a 'lifestyle-affirming' migration in-flow of middle-lifers who are seeking seaside destinations that align with their personality, where they have brought their previous habitus and life experiences into an environment that is stable, quiet, safe and unchanging and best suits their social class disposition. This clustering force has resulted in the geographic concentration of personality types, and such mobility flows have been shown elsewhere to reshape the social, economic and cultural ecology of resorts (Coles and Timothy, 2004; Agarwal and Brunt, 2006). Thomas and Dorling (2007) and Florida (2008) contend that while opposites sometimes attract, most people are happier and more fulfilled in places that in some sense match their personalities. However, the statutory key agencies acknowledged how their past collective actions have shaped the kinds of people they attracted, which appears to be an important point for English seaside resort towns. The value that migrants place on places, and how people perceive destinations, is subject to a complex interface between people's previous experiences, cultural imaginings, individual circumstances, including their education, class disposition and economic capital, and certain physical, historical and material conditions at the point of migration that contributed to how they perceive a place. This inmigration, whilst a deliberate act was informed by the attraction of clustering with likeminded people and personalities in a physical environment that reflected the same. The town of Bexhill-on-Sea attracted middle-life migrants whose decisions are based on information gathered from personal experiences elsewhere that they imprinted on the town and from images and labels that were attached to the town such as 'Costa Geriatrica' and 'Retirement Coast'. Their choice of destination was based on a perceived image, gut-feel and how 
the in-migrants would best 'fit-in'. This 'lifestyle-affirming' migration and 'personality fit' was a significant and key finding in the research, thus uncovering interconnections between the physical environment, key agencies and middle-life people living in the destination, as well as the images and labels that were attached to Bexhill-on-Sea.

The research has therefore shown that middle-life migration patterns can shape a destination. However, resorts are fashioned by the physical and natural environments, which in turn influence people who visit, migrate to and live in them (Agarwal, 2002; Ravenscroft and Hadjihambi, 2006; Agarwal and Shaw, 2007; Benedict and Houghton, 2009) and out of which images and labels are shaped and formed which set the direction for the seaside resort town. The research evidences the role of agency as having a significant utility regarding the structure of the town, and key agency actions exerted a significant influence on the physical environment, which in turn influences the resort structure. This utility of agency is also shown in the case study through the behaviours and choices of middle lifers who are able to influence the cultural meme and personality of the destination. Agency, therefore, can alter the physical environment, adapt demographics and change the image - and thus the socioeconomic and cultural performance - of the place.

\subsection{Conclusion}

The purpose of this thesis was to address problems related to the apparent decline in seaside resort towns and to contribute to the body of knowledge about resort regeneration. Although the thesis has drawn attention to interconnections between the physical environment, key agency actions and middle-life migration in the regeneration of seaside resorts, it is nevertheless cautious about privileging these processes. The narrative research did not attempt to create any conclusions based on certainty, but aimed for its findings to be 'well-grounded' and 'supportable', backed up with rigour of application to offer credibility and authenticity, so as to add insight to the complex forces at work in resorts. This thesis argues that the single-case study using narrative inquiry offered a focus on a specific case that uncovered the uniqueness and complexity of the locality and aligned with the aims and objectives of this 
research. This in turn offers interpretations but does not claim to represent the exact truth, and so the research remains open-ended and opens up possible areas of further research.

By bringing together the analytical findings with the literature review, the thesis has addressed a problem by way of identifying knowledge gaps in the current understanding of seaside resort towns. The data suggests that while the resort development process is largely externally initiated and mediated (Butler, 2006a; 2006b), its adaptation can be profoundly influenced by endogenous forces, as discussed by Agarwal $(1997,1999,2006)$ and Cooper $(1997,2006)$. The primary and secondary research findings support the literature review that there are many complex forces at work in resorts, and they evidence the role of agency as having a utility in which the energy that key agency actions exerted on the physical environment has influenced the resort structure. The thesis argues that agency at the local level may not be able to overcome these external forces, but it could influence change locally, whether intentionally or otherwise. The actions taken by key agencies within the resort of Bexhill-onSea have altered its structure and shaped the culture and personality of the place as well as its socio-economic performance. From an evolutionary perspective, interconnections between the physical environment and the socioeconomic structure have been altered through exogenous forces and the endogenous actions of agency, thus affecting migration to and from the resort and the consequent repositioning of how it is perceived and interpreted by migrants.

An increasing number of seaside resort towns appear to be facing rather uncertain futures, and the decline (in the absence of intervention) described by Butler's (1980) TALC model may well materialise in some seaside locations. The complex evolution of resorts varies depending, but not exclusively, on aspects such as the performance of the national economy, housing, amenities, access, local circumstances and politics (Butler, 2006a), which was supported by the findings of the research. As identified throughout the thesis, macrostructural conditions are important, because they function as structures that constrain or enable development from outside (Johnston, 2006). However, endogenous actions, particularly the power of institutional and individual agency 
as described by Zimon (2000), Agarwal $(2002,2007)$ and Ravenscroft and Hadjihambi (2006), further our awareness of how resorts respond to these influences.

The reduction in tourism facilities and the change towards retirement services have transformed the socio-economic and cultural structure of resorts. The role of elected officials in the evolution of these places during the past few decades of stagnation and decline has evidenced the importance of the role and function of agency. These interconnections and interrelations between the physical environment, key agencies and middle-life people were striking in the case study. Elected officials were identified as the most important (cultural) component of the resort system, such that they guide the 'meme'. The research corroborated the literature findings, in that resorts are not passive victims of global forces, and their evolution is also determined by the impact of the interconnections of key agencies, local politics and leadership that can reform the social ecology of resorts and their lifecycle. It is these local responses and interactions with broader external forces that have produced place-specific outcomes for Bexhill-on-Sea.

Critical within the thesis was the recognition of the emergent 'New Economy' and how future retirement patterns may lead people to react differently to the past, as middle-lifers have come through a consumer society and different life courses and as the future nature of work changes. The expectation taken from the literature review was that the research findings would show a middle-life migration in search of work or a second act (Moen, 2005). But the case study evidenced that this was not a prevalent finding, as the middle-life migrants moving to Bexhill-on-Sea, in the main, were not in search of work but instead a quiet, non-vibrant place offering all the basic amenities in which to live out retirement. They stated that they were not looking for work and that the lack of employment and economic activity helped keep the town tranquil. This finding of middle-life in-migrants looking for non-vibrant seaside places that best suited their personality was a critical finding, in that it may also be important for regeneration practitioners in determining and identifying where clusters of middle-lifers may choose to locate in future. 
Amongst structural exogenous forces, the actions of key agencies can direct a set of local socio-economic and cultural 'circonstances' (Butler, 1914) for the town through their decision-making and imposition on the physical environment. In this respect, Lamarck's work accepts the environment as one of the principal catalysts for change and adaptation. As a result, the local authority, as a direct result of its intervention, is seeking to move the population and its meme towards a new environment and culture where socio-economic improvement occurs. However, achieving this aim requires changing the cultural meme of the resident and business population of the town and repositioning its image to appeal to different individuals.

The thesis has highlighted opportunities for further research into socioeconomic and cultural change in resorts by challenging conventional thinking on the resort lifecycle, resort evolution and the role of retirement migration behaviours. The research leaves open the need for further case studies that fully understand the role of key agencies, in-migration and those long-standing residents, all of whom may have a very different view and perception on the role of tourism, employment and regeneration. The work also identifies how regeneration practitioners might undertake in-depth research into migration, to better understand the dynamics of their locality, and how regeneration projects and proposals may be received within communities. The thesis evidenced a key finding of the in-migration of middle-lifers, who were not seeking a change in lifestyles but were instead affirming their lifestyle and class disposition. Further research into lifecycle and lifestyle migration would contribute to this knowledge and understanding.

This research has added to new knowledge about the interconnections between the physical environment, key agencies and middle-life migration and informs studies of the regeneration challenges in resort seaside towns. The findings arising from this study are valuable but whilst this thesis promoted the justification for the use of a single case study to understand uniqueness, contextuality and complexity of the individual case there are implications arising from the research that could be tested elsewhere. For instance, future research could investigate the ideas in a wider array of coastal towns along the south coast, such as Eastbourne, Bognor Regis or Bournemouth. The study could 
also look at seaside resorts with a high elderly population in the south west, such as Sidmouth, Dawlish or St Ives, or to the north of England such as Skegness or Bridlington. By contributing to wider generalisations this may help remove fears about uniqueness or artificial conditions surrounding the single case study and further research could be compared against some of the findings in this thesis.

The implications for future research of this thesis could be profound in that it provides evidence that places like people have personalities and that this plays a role in people clustering together. This may offer insights as to why some resorts succeed and others fail when they have been subject more or less to the same set of exogenous forces. The study of Margate and the clustering of benefit claimants may not therefore just be related to accessibility to affordable housing but may also be related to a clustering of likeminded people that requires more detailed examination.

The research identified the importance of key agency actions and especially those of the elected officials. This could be usefully examined in future case studies and the interrelationship between paid and elected officials examined in greater depth to gain a better understanding of key agency roles, accountability and decision-making. Exploring the politics and leadership roles associated with the transformation of Bexhill-on-Sea has added further knowledge to the case study and resort lifecycles and may aid regeneration practitioners. This Lamarckian perspective of agency being influential on the environment requires further research in other locations. Also the findings in this research concerning the nature of the relationships between employers and officials in the area of employment require further analysis and understanding both in the case study and elsewhere. The thesis revealed differing opinions depending on the employment sector the participants represented. There was evidence that the retail sector aligned with the middle-life participants while a portion of other employment sectors, such as industry, offered a different view of the town and its socio-economic performance that supported more the key statutory agencies perspectives. Examining this amongst employers and other resorts would prove valuable. Finally, the implications of the perceptions of images and labelling of the place and different interpretations of 'Costa Geriatrica' may 
require a different research approach perhaps using images to stimulate interview discussion or the use of focus groups in gathering data to reveal participants' values and behaviours associated with such labels both living within the town or those who would not seek to migrate to Bexhill-on-Sea.

The decision to focus on a single case study was determined in order to understand uniqueness, contextuality and complexity of the individual case. Some researchers Yin (2009), Bryman (2004) consider the limitations of the single-case study strategy in that it may only reflect uniqueness or artifactual conditions relating only to the case study in question. Therefore generalising findings beyond the immediate case study is not feasible and which may result in uncertainty of the empirical research being conducted. Multiple-case study supporters Clandinin, (2007), Hyde et al., (2012) claim that this form is likely to be more useful than the single-case study, as it is believed that the evidence from multiple-case studies is often considered more compelling. If this view is supported then recognising this limitation and that the findings reflect localebased behaviour then transposing these findings onto other resorts would be inappropriate. However, this research offers valuable insights into the interconnections of the physical environment, key agencies and middle-life migration and it is recommended that further locality studies need to be undertaken that may build on this research.

The study interviewed middle-life migrants aged between 50-70 years of age that could be viewed as a constraint of the study in that the research did not attempt to analyse the views of differentiated age groups within this middle-life age range. The study identified a migration of 'lifestyle-affirming' and likeminded personality clustering and that this internal homogeneity appeared to prevail across the age bands and the research did not reveal any age related perspectives. However, this was not tested for and therefore could be usefully examined in further research. The interviews with the middle-life migrants also excluded those who were in the same age category but had resided in the town for many years, which may have offered a different perspective as to why they live in the town and how they may have influenced socio-economic change in the resort. Neither did the research capture the perceptions of those who may have considered migrating to Bexhill-on-Sea but choose not to do so. In both 
these instances it was considered unachievable to research due to the need to keep the sample size to a manageable level. Further research into these other groups may offer insights as to why these people remained in the town for longer than five years and why those who chose not to migrate to Bexhill-onSea but instead chose another resort location in which to retire. The research also did not seek to address the views of those middle-lifers who were outmigrating that may have added value to the research to understand their reasons and motivations for leaving Bexhill-on-Sea.

The research identified the importance of past key agency actions, in particular elected officials, and the impact their agency decisions has had on the structure of the town. The research also showed there was conflict and difference of opinion amongst the elected officials over the recent attempts at regeneration projects in the town. If the research had been able to interview all 38 of the elected officials who represented the District, especially the local ward representatives for Bexhill-on-Sea, it may have generated further valuable findings and presented additional knowledge on seaside resort town's future regeneration. However, this was not feasible to undertake due to the capacity of the researcher and the need given the aims of the thesis to also capture and understand wider non-statutory key agency and middle-life migrants' perceptions.

In conclusion, the thesis has advanced knowledge of the multifarious and diverse processes involved in a resort's lifecycle and its regeneration, by unravelling the complex links between the physical environment, key agencies and middle-life migration. The research evidenced that resorts are not passive victims of global forces but are strongly influenced by the outcome of the quality of agency actions. Many resorts seem to have undergone transitions that appear 'blurry' and complex and have altered the socio-economic and cultural structure of the destination. These alterations in the environment and adaptation of people migrating, and the clustering of likeminded personalities, have resulted in changing perceptions of the place through the labels and images they create. The conceptual juncture between human economic, social and cultural capital and the physical environment has been neglected by each individual research agenda, but by engaging with the debates a new 
perspective is gained which questions dominant theoretical understandings of resorts' middle-life migration and regeneration processes.

By fully exploring a seaside town case study, the thesis argues how the physical environment, key agency actions, middle-life migration and image construct new temporal-spatial patterns which inform an interesting research agenda for retirement migration and regeneration. The utility of agency is able to influence the cultural meme of the destination, but success for the leadership requires a supportive governance, business and community culture which allows for altering the physical environment, adapting human social, cultural and economic capital and changing the image. The case study has shown that the introduction of change through turbulent and municipal revitalising entrepreneurialism can create opposition, as retired middle-lifers and those businesses servicing the retired resist change in an attempt to keep the town's personality as it is - namely, a tranquil, safe, non-vibrant, non-economic 'Costa Geriatrica'.

This thesis has challenged conventional understanding of resorts and of seaside town regeneration, by revealing interconnections between processes of change in the physical environment and key agency actions and middle-life 'retirement' migration. In so doing, it has uncovered a unique perspective and added new knowledge to the understanding of a seaside town's lifecycle and revival. This thesis transcends the relatively one-dimensional theorisation of resort retirement migration based on nostalgia, and instead it advocates the significance of personality motives and clustering within retirement migration in physical environments that best suit migrants' personality. A number of seaside resorts appear to be at a critical juncture, and Bexhill-on-Sea's political leadership's strategic direction between the status quo and regeneration will continue to be played out, thereby leaving an uncertain future for the town. 


\section{Bibliography}

AARP: American Association of Retired Persons, (2002) Global Aging: Achieving lts Potential

Washington: AARP Public Policy Institute Retrieved from

www.aarp.org_/articles/international/global_aging.pdf

AARP: American Association of Retired Persons, (2003) AARP Policy Book Chapter 4 Employment

Washington: AARP Public Policy Institute

Abercombie, N. and Warde, A., (2000) Contemporary British Society ( ${ }^{r d}$ ed.)

Cambridge: Polity Press

Ackers, L. and Dwyer P., (2002) Senior Citizenship? Retirement Migration and Welfare in the European Union

Bristol: Policy Press

Adams, Thomson and Fry, (1930) Borough of Bexhill Planning Scheme: Report on the General Development Plan

London (City): F.J. Parsons

Adams-Acton, M., (2010) Economic Indicators and Trends in Hastings Borough Hastings Borough Council

Agar, M., (1986) Speaking of Ethnography

London: Sage

Agarwal, S.J., (1994) The Lifecycle Approach and South Coast Towns In Cooper, C. and Lockwood, A., (eds.) Progress in Tourism Recreation and Hospitality Management, Vol no. 5

Chichester: Wiley

Agarwal, S.J., (1995) The Resort Cycle and Restructuring: The Case of Coastal Tourism in the South of England Faculty of Social Studies, PhD Thesis, advisors: Shaw, G. and Williams, A.

University of Exeter

Agarwal, S.J., (1997) The Resort Cycle and Seaside Tourism: An assessment of its Applicability and Validity Tourism Management, Vol no. 18, Issue 2, March 1997, pp. 6573

Agarwal, S.J., (1999) Restructuring and Local Economic Development: Implications for seaside resort regeneration in Southwest Britain

Tourism Management, Vol no. 20 (4), pp. 511-522

Agarwal, S.J., (2002) Restructuring Seaside Tourism: the Resort Lifecycle Annals of Tourism Research, Vol no. 29 (1), pp. 25-55

Agarwal, S.J., (2005) Global interactions in English coastal resorts: Theoretical

Perspectives Tourism Geographics, Vol no. 7 (4), pp. 351-372

Agarwal, S.J., (2006) Coastal Resort Restructuring and the TALC Model In Butler R.W. (ed.) The Tourism Area Life Cycle, Vol. 2, pp. 201-218

Clevedon: Channel View Publications

Agarwal, S.J., (2007) Institutional Change and Resort Capacity: The Case of Southwest English Coastal Resorts In Agarwal, S. and Shaw, G. (eds.). Managing Coastal Resorts: a Global Perspective, pp. 56-70

Clevedon: Channel View Publications

Agarwal, S. and Brunt, P., (2006) Social Exclusion and English Seaside Resorts 
Tourism Management, Vol no. 27, pp. 654-670

Agarwal, S. and Shaw, G., (2007) Managing Coastal Tourism Resorts - A Global

Perspective

Clevedon: Channel View Publications

Aguiló, E., Alegre, J. and Sard, M., (2005) The Persistence of the Sun and Sand Tourism Model Tourism Management, Vol no. 26, pp. 219-231

Alcock, P., Beatty, C., Fothergill, S., Macmillan, R. and Yeandle, S., (2003) Work to Welfare: How Men Become Detached from the Labour Market

Cambridge: Cambridge University Press

Ambrose, P., (2010) The Great Financial Crisis - why it happened and what to do about it Seminar held in April 2010 by Coastal Research Centre

University of Brighton

Andrews, M., (1999) The seductiveness of agelessness Ageing and Society, Vol no. 19, pp. 301-18

Appleby, J., (1993) Consumption in Early Modern Social Thought In Brewer, J. and Porter, R., (eds.) Consumption in World of Goods, pp. 162-173

London: Routledge

Argus, (2005, April 5) The Ad men reinvent Gods Waiting Room Retrieved from www.theargus.co.uk/2004/4/5/115882.html

Argus, (2009, July 29) It's time to say goodbye to the Costa Geriatrica Retrieved from www.theargus.co.uk/news/commentandanalysis/45176227.It_s_time_to_say_goodbye_t o_costa_geriatrica/

Arkenford Consultancy, (2007) Hastings and 1066 Country Visitor Study: Hastings Destination Profile: Bexhill-on-Sea Destination Profile Arkenford Marketing Modelling and Research Study

http://www.rother.gov.uk/media/pdf/3/q/Hastings_and_1066_Country_Ark_segmentation _research_07.pdf

Arts Council England, South East, (2006) Our Agenda for the Arts in the South East 2006/08

Revolution: Aldridge Print Group

Atkinson, R. and Moon, G., (1994) Urban Policy in Britain: The City, the State and the Market

London: Macmillan Press

Atterton, J., (2006) Ageing and Coastal Communities: Final Report for the Coastal Action Zone Partnership

Newcastle upon Tyne: Centre for Rural Economy

Audit Commission, (2004) Older People - Independence and well-being: The challenge for public services

Audit Commission and Better Government for Older People

http://archive.audit-commission.gov.uk/auditcommission/sitecollection

documents/AuditCommissionReports/NationalStudies/OlderPeople_overarch.pdf

Autor, D.H. and Duggan, M.G., (2003) The rise in the Disability Rolls and the Decline in Unemployment Quarterly Journal of Economics, Vol no. 118, pp. 157-206

Bailey, N. and Livingston, M., (2007) Population Turnover and Area Deprivation

York: Joseph Rowntree Foundation

Bailey, N., Haworth A., Manzi, T., Paranagamage, P. and Roberts, M., (2006) Creating and Sustaining Mixed Income Communities: A Good Practice Guide 
York: Joseph Rowntree Foundation

Baines, S., Lie, M. and Wheelock, J., (2004), Volunteering, self-help and citizenship in later life A collaboration research project by Age Concern Newcastle and the University of Newcastle upon Tyne

Baker, R., (2005) Age discrimination and bio-medicine, speech to Council of Europe Ethics Committee Conference, Dubrovnik (April)

Baker, R. and Speakman, L., (2006) The Older Rural Consumer In Lowe P. and Speakman L., (eds.) The Ageing Countryside: The Growing Older Population of Rural England, pp. 119-132

London: Age Concern

Baltes, M. and Carstensen, L., (1996) The Process of Successful Ageing Ageing and Society, Vol no. 16 (4), pp. 397-422

Bartley, L.J., (1971) The Story of Bexhill

Bexhill on Sea: F.J. Parsons Ltd

Barnes, H., Parry, J. and Taylor, T., (2004) Working after State Pension Age: Qualitative Research DWP Research Report No. 208

Baudrillard, J., (1998) The Consumer Society: Myths and Structures London: Sage

Baum, T., (1998a) Taking the exit route: Extending the tourism area life cycle model Current Issues in Tourism, Vol no. 1 (2), pp. 167-175

Baum, T., (1998b) Tourism marketing and the small island environment. Cases from the periphery In Laws, E., Faulkner, B. and Moscardo, G., (eds.) Embracing and Managing Change in Tourism London: Routledge

Baum, T., (2006) Revisiting the TALC: Is there an off-ramp? In Butler R.W., (ed.) The Tourism Area Life Cycle, Vol no. 2, pp. 219-233

Clevedon: Channel View Publications

Bauman, Z., (1998) Consumerism and the New Poor

Milton Keynes: Open University Press

Bauman, Z., (2000) Liquid Modernity

Cambridge: Polity Press

BBC News, (2005, September 26) Prison Tax Protest Pensioner Retrieved from http://news.bbc.co.uk/1/hi/england/devon/4280040.stm

BBC News, (2006, August 27) A Growing and Ageing Nation Retrieved from http://newsvote.bbc.co.uk/mpapps/pagetools/print/news.bbc.co.uk/1/hi/uk/5282374.stm

BBC Inside Out, (2010, December 6) High street winners and losers revealed in BBC survey Retrieved from

http://news.bbc.co.uk/local/manchester/hi/people_and_places/newsid_9260000/926072 6.stm

BBC News, Education and Family (2012, March 21) Secondary School League Tables in East Sussex Retrieved from

http://www.bbc.co.uk/news/special/education/school_tables/secondary/11/html/845.stm

Beatty, C. and Fothergill, S., (2002) Moving Older People into Jobs: Jobcentre Plus,

New Deal and the job shortfall for the over 50 s

London: Third Age Employment Network 
Beatty, C. and Fothergill, S., (2003) The Seaside Economy: The final report of the seaside towns research project Centre for Regional Economic and Social Research, Sheffield Hallam University

Beatty, C. and Fothergill, S., (2004) Economic Change and the Labour Market in Britain's Seaside Towns Regional Studies, Vol no. 38 (5), pp. 459-478

Beatty, C. and Fothergill, S., (2007) Changes in the Profile of Men Claiming Incapacity Benefit - a case study People, Place \& Policy Online, Vol no. 1/3, pp. 136-148

Beatty, C., Fothergill, S. and Barraclough, N., (2003) Unemployment and Economic Inactivity in Britain's Seaside Towns Working Paper No 2, Seaside Towns Research Project. Centre for Regional and Economic and Social Research, Sheffield Hallam University

Beatty, C., Fothergill, S., Powell, R. and Wilson I., (2006) Coastal Proofing the Benefits System: A Report to Lincolnshire Coastal Action Zone Sheffield: Centre for Regional Economic and Social Research, Sheffield Hallam University

Beatty, C., Fothergill, S. and Wilson, I., (2008) England's Seaside Towns, A Benchmarking Study Department for Communities and Local Government, Centre for Regional Economic and Social Research, Sheffield Hallam University

Beatty, C., Fothergill, S. and Gore, A., (2009) Seaside Tourism Industry - Employment, Economic Output, Location and Trends (Sheffield Hallam University Meeting $2^{\text {nd }}$ December 2009)

Beatty, C., Fothergill, S., Gore, A. and Wilson, I., (2012) The Coastal Communities of South East England: A Statistical Profile of the Coastal Communities within the South East LEP A report to the coastal local authorities in the South East LEP - April 2012 Centre for Regional Economic and Social Research, Sheffield Hallam University

Beauregard, R.A., (1986) The Chaos and Complexity of Gentrification In Smith, N. and Williams, P., (eds.) Gentrification of the City Winchester MA, pp. 35-55 Allen and Unwin

Begg, I., Moore, B.C. and Rhodes, J., (1986) Economic and Social Change in Urban Britain and the Inner Cities In Hausner V., (ed.) Critical Issues in Urban Economic Development Clarendon Press: Oxford

Bell, D. and Valentine, G., (1997) Consuming Geographies: We Are Where We Eat London: Routledge

Benedict, R. and Houghton, J., (2009) Solving the Riddle of the Sands: Regenerating England's Seaside Towns Journal of Urban Regeneration and Renewal Issue, Vol no. 3, Number 1 July - September 2009

Benson, M., (2007) There's more to life: British lifestyle migration to rural France PhD Thesis, Comparative and Applied Social Sciences, University of Hull

Benson, M. and O'Reilly K., (2009) Migration and the search for a better way of life: a critical exploration of lifestyle migration

The Sociological Review, Vol no. 57 (4), pp. 608-625

Benson, M. and O'Reilly, K., (2009a) Lifestyle Migration: Expectations, Aspirations and Experiences Studies in Migration and Diaspora

Ashgate: Surrey

Bernard, M. and Phillips, J., (2000) The challenge of Ageing in Tomorrow's Britain Ageing Society, Vol no. 20, pp. 33-54

Berry, T., (2006) The predictive potential of the TALC model In Butler R.W., (ed.) The Tourist Area Life Cycle, Vol no. 2: Conceptual and Theoretical Issues, pp. 254-279 
Clevedon: Channel View

Bertolini, L., (2007) Evolutionary Urban Transportation Planning: An Exploration

Environment and Planning, Vol no. 39 (8), pp. 1998-2019

Bexhill Hanoverian Study, (2003) The King's German Legion from Bexhill to the Battle of Waterloo

Bexhill Museum, (2010a) A Summer Memory of Bexhill

Film archive only on display at Bexhill Museum

Bexhill Museum, (2010b) The History of Bexhill Retrieved from

www.bexhillmuseum.co.uk/museum-pages/the-history-of-bexhill-48.html/ the-de-la-warrpavilion-59html

Bexhill Observer, (2004, April 15) Seafront hotel protest group formed Retrieved from http://www.bexhillobserver.net/news/bexhill-news/rally-by-seafront-hotel-protesters-11403530

Bexhill Observer, (2004, December 23) Save our sea view Retrieved from http://www.bexhillobserver.net/news/bexhill-news/save-our-sea-view-1-1403852

Bexhill Observer, (2008, July 30) We want motor heritage built into scheme, say traders Retrieved from

http://www.bexhillobserver.net/news/bexhill-news/we-want-motor-heritage-built-intoseafront-scheme-say-traders-1-1399383

Bexhill Observer, (2008, August 1) Consultation needed over $£ 5.1 \mathrm{~m}$ seafront plan says scrutiny committee Retrieved from

http://www.bexhillobserver.net/news/bexhill-news/consultation-needed-over-163-5-1mseafront-plan-says-scrutiny-committee-1-1399395

Bexhill Observer, (2008, August 18) Planning committee's concern over Colonnade plan Retrieved from

http://www.bexhillobserver.net/news/bexhill-news/plannning-committee-s-concern-overcolonnade-plan-1-1399541

Bexhill Observer, (2008, September 3) Seafront scheme 'misunderstood' - Rother Retrieved from

http://www.bexhillobserver.net/news/bexhill-news/seafront-scheme-misunderstoodrother-1-1399655

Bexhill Observer, (2008, September 3) Rother sets out seafront questions Retrieved from

http://www.bexhillobserver.net/news/bexhill-news/rother-sets-out-seafront-questions-11399679

Bexhill Observer, (2008, September 23) Council 'must listen' on regeneration Retrieved from

http://www.bexhillobserver.net/news/bexhill-news/council-must-listen-on-regeneration-11399797

Bexhill Observer, (2008, October 21) £1m grant boost for Rother Next Wave project Retrieved from

http://www.bexhillobserver.net/news/bexhill-news/163-1m-grant-boost-for-rother-nextwave-project-1-1399995

Bexhill Observer, (2008, October 24) Seafront future - a lesson in history Retrieved from http://www.bexhillobserver.net/news/bexhill-news/seafront-future-a-lesson-in-history-11400039

Bexhill Observer, (2008, December 4) Seafront Improvement plan Retrieved from http://www.bexhillobserver.net/news/bexhill-news/seafront-improvement-plan-1-1400359 
Bexhill Observer, (2009, March 27) Anger erupts at town meeting over seafront Retrieved from http://www.bexhillobserver.net/news/bexhill-news/anger-erupts-at-town-meeting-overseafront-1-1400631

Bexhill Observer, (2009, April 29) Council invite business interest in Colonnade Retrieved from http://www.bexhillobserver.net/news/bexhill-news/council-invites-business-interest-incolonnade-1-1400841

Bexhill Observer, (2009, June 9) Architect is named for seafront project Retrieved from http://www.bexhillobserver.net/news/bexhill-news/architect-is-named-for-seafrontproject-1-1401095

Bexhill Observer, (2009, July 7) Cabinet vote on Next Wave design Retrieved from http://www.bexhillobserver.net/news/bexhill-news/cabinet-vote-on-next-wave-design-1. 1401267

Bexhill Observer, (2009, August 13) Protest over seafront bench plans Retrieved from http://www.bexhillobserver.net/news/bexhill-news/protest-over-seafront-bench-plans-1. 1401440

Bexhill Observer, (2009, August 20) Memorial benches: donors reveal thoughts on Next Wave scheme Retrieved from

http://www.bexhillobserver.net/news/bexhill-news/memorial-benches-donors-revealthoughts-on-next-wave-scheme-1-1401472

Bexhill Observer, (2009, September 15) Next Wave exhibition at Bexhill Museum Retrieved from http://www.bexhillobserver.net/news/bexhill-news/next-wave-exhibition-at-bexhillmuseum-1-1401574

Bexhill Museum, (2009, September 23) Next Wave exhibition Retrieved from http://www.bexhillobserver.net/news/bexhill-news/next-wave-exhibition-1-1401615

Bexhill Observer, (2009, September 29) Next Wave: Councillors divided over party lines Retrieved from http://www.bexhillobserver.net/news/bexhill-news/next-wave-councillors-divided-overparty-lines-1-1401649

Bexhill Observer, (2009, October 29) Bexhill residents want further consultation on Next Wave development Retrieved from http://www.bexhillobserver.net/news/bexhill-news/bexhill-residents-want-furtherconsultation-on-next-wave-development-1-1401822

Bexhill Observer, (2010, January 7) 2009 review part II: August-December Retrieved from http://www.bexhillobserver.net/news/bexhill-news/2009-review-part-ii-august-december$1-1401988$

Bexhill Observer, (2010, January 15) Week one: Bexhill Rowing Club and the Colonnade Retrieved from http://www.bexhillobserver.net/news/bexhill-news/week-one-bexhill-rowing-club-and-thecolonnade-1-1402030

Bexhill Observer, (2010, January 21) Council backtrack over controversial cupola Retrieved from http://www.bexhillobserver.net/news/bexhill-news/council-backtrack-over-controversialcupola-1-1402063

Bexhill Observer, (2010, January 28) Week three: Bexhill's roads Retrieved from http://www.bexhillobserver.net/news/bexhill-news/week-three-bexhill-s-roads-1-1402093 
Bexhill Observer, (2010, February 18) Go-ahead for first wave of seafront plan Retrieved from http://www.bexhillobserver.net/news/bexhill-news/go-ahead-for-first-wave-of-seafrontplan-1-1402156

Bexhill Observer, (2010, April 15) Next Wave project breaks new ground Retrieved from http://www.bexhillobserver.net/news/bexhill-news/next-wave-project-breaks-newground-1-1402340

Bexhill Observer, (2010, June 17) Town Centre refit: plans unveiled Retrieved from http://www.bexhillobserver.net/news/bexhill-news/town-centre-refit-plans-unveiled-11402607

Bexhill Observer, (2010, September 30) Protest rally at town hall Retrieved from http://www.bexhillobserver.net/news/bexhill-news/protest-rally-at-town-hall-1-1593741

Bexhill Observer, (2011, January 30) Vandals hit Next Wave benches Retrieved from http://www.bexhillobserver.net/news/bexhill-news/vandals-hit-next-wave-benches-12351552

Bexhill Observer, (2011, February 5) Pavilion public funding goes under microscope Retrieved from http://www.bexhillobserver.net/news/bexhill-news/pavilion-public-funding-goes-undermicroscope-1-2382600

Bexhill Observer, (2011, August 21) Scale shelters design challenge for youngsters Retrieved from http://www.bexhillobserver.net/news/bexhill-news/scale-shelters-design-challenge-foryoungsters-1-2979380

Bexhill Observer, (2011, September 2) Next phase of resurfacing work Retrieved from http://www.bexhillobserver.net/news/bexhill-news/next-phase-of-resurfacing-work-13018749

Bexhill Observer, (2011, December 28) Colonnade traders say they want to stay Retrieved from

http://www.bexhillobserver.net/news/bexhill-news/colonnade-traders-say-they-want-tostay-1-3360875

Bexhill Observer, (2012, February 10) Special meeting for £5.6m Next Wave scheme Retrieved from http://www.bexhillobserver.net/news/bexhill-news/special-meeting-for-5-6m-next-wavescheme-1-3505928

Bexhill Observer, (2012, April 6) £5.6m seafront scheme is hotly debated Retrieved from http://www.bexhillobserver.net/news/bexhill-news/5-6m-seafront-scheme-is-hotlydebated-1-3706673

Bexhill Observer, (2012, June 4) Shelters undergo improvements due to weathering Retrieved from http://www.bexhillobserver.net/news/bexhill-news/shelters-undergo-improvements-dueto-weathering-1-3905860

Bexhill Observer, (2012, June 29) Royalty to officially open Diamond Jubilee Walk Retrieved from http://www.bexhillobserver.net/news/bexhill-news/royalty-to-offically-open-diamondjubilee-walk-1-4003124

Bexhill Observer, (2012, July 16) Seafront wins top award for design Retrieved from http://www.bexhillobserver.net/news/bexhill-news/seafront-wins-top-award-for-design-14051351 
Bexhill Observer, (2012, September 4) Rotary sundial finds brand new home at Colonnade Retrieved from

http://www.bexhillobserver.net/news/bexhill-news/rotary-sundial-finds-brand-new-homeat-colonnade-1-4214318

Bexhill Observer, (2013, March 1) Sea change for Bexhill is hailed a winner by Rother Council Retrieved from

http://www.bexhillobserver.net/news/bexhill-news/sea-change-for-bexhill-is-hailed-a-

winner-by-rother-council-1-4843804

Bexhill Observer, (2005, February 23) Seafront hotel project is ditched Retrieved from http://www.bexhillobserver.net/news/bexhill-news/seafronot-hotel-project-is-ditched-11403942

Bexhill Observer, (2010, May 21) Letters to the Editor Retrieved from http://www.bexhillobserver.net/

Bianchini, F., Dawson, J. and Evans, R., (1992) Flagship Projects in Urban Regeneratiom In Healey, P., O'Toole, S.D., Tavsanoglu, S. and Usher, D., (eds.).

Rebuilding the City, pp. 245-255

London: E\&FN SPON

Blow, L., Leicester, A. and Oldfield, Z., (2004) Consumption Trends in the UK 1975-99 The Institute for Fiscal Studies

Glasgow: Bell and Bain Ltd

Boaz, A., Hayden, C. and Bernard, M., (1999) Attitudes and Aspirations of Older People: A review of the literature Department of Social Security (DSS) Research Report No.

101, Leeds, CDS

Huddersfield: The Charlesworth Group

Boesiger, E., (1974) Evolutionary Theories after Lamarck and Darwin In Ayala, F.J. and Dobzhansky, T.G., (eds.) Studies in the Philosophy of Biology: Reduction and Related Problems, pp. 21-44

London: Macmillan

Boorman, K., (2009) Hastings Borough Council, Closure of Hastings Pier - Issues, options and scenarios (Personal communication $-2^{\text {nd }}$ December 2009)

Booth, P. and Boyle, R., (1993) See Glasgow, see Culture In Bianchini, F. and Parkinson, M., (eds.) Cultural Policy and Urban Regeneration: The West European Experience, pp. 21-47

Manchester: Manchester University Press

Bottero, W., (2000) Gender and the Labour Market at the Turn of the Century:

Complexity, Ambiguity and Change

Work, Employment and Society, Vol no. 14 (4), pp. 781-791

Bourdieu, P., (1984) Distinction: A Social Critique of the Judgement of Taste (translated by Richard Nice)

London: Routledge and Kegan Paul

Bourdieu, P., (1990) The Logic of Practice (translated by Richard Nice)

Stanford, California: Stanford University Press

Boyle, D. and Harris, M., (2009) The challenge of co-production: how equal partnerships between professionals and the public are crucial to improving public services London: Nesta and nef

Bramwell, B., (2004a) Coastal Mass Tourism Diversification and Sustainable

Development in Southern Europe

Clevedon: Channel View Publications 
Bramwell, B., (2004b) The policy context for tourism and sustainability in Southern

Europe's coastal regions In Bramwell B., (ed.) Coastal Mass Tourism Diversification and Sustainable Development in Southern Europe, pp. 32-47

Clevedon: Channel View Publications

Bramwell, B., (2004c) Mass tourism, diversification and sustainability in southern

Europe's coastal regions In Bramwell B. (ed.) Coastal Mass Tourism: Diversification and

Sustainable Development in Southern Europe, pp. 1-31

Clevedon: Channel View Publications

Brett, J.M. and Stroh, L.K., (2003) Working 61 hours plus a week: Why do managers do it? Journal of Applied Psychology, Vol no. 88, pp. 67-78

Brewer, J.D., (2000) Ethnography In Understanding Social Research series Editor Alan Bryman

Buckingham, Philadelphia: Open University Press

British Tourist Authority, (1975) Resorts and Spas in Britain London: BTA.

Brodie, A., Sargent, A. and Winter, G., (2005) Seaside Holidays of the Past National Monuments, Records

London: English Heritage

Brodie, A. and Winter, G., (2007) England's Seaside Resorts

Swindon: English Heritage

Bromley, R.D.F., Tallon, A.R. and Roberts, A.J., (2007) New populations in the British city centre: evidence of social change from the census and household surveys Geoforum, Vol no. 38, pp. 138-154

Brook, J., (1997) The Story of the De La Warr Pavilion In A Touring Exhibition in Erich Mendelsohn 1887-1953: A touring exhibition organised by Modern British Architecture Bexhill-on-Sea: Modern British Architecture in conjunction with A3 Times

Bryman, A., (1988) Quantity and Quality in Social Research In Bryman, A., Social Research Methods

London: Unwin Hyman

Bryman, A., (2004) Social Research Methods ( $2^{\text {nd }}$ ed.)

Oxford: Oxford University Press

Bryman, A., (2006) Integrating Quantitative and Qualitative Research: how is it done? Qualitative Research, Vol no. 6 (1), pp. 97-113

Bryman, A., (2007) Barriers to Integrating Quantitative and Qualitative Research Journal of Mixed Methods Research, Vol no. 1 (1), pp. 8-22

Bucks, N., Gordon, I., Pickvance, C. and Taylor-Goodby, P., (1989) The Isle of Thanet: restructuring and municipal conservatism In Cooke, P., (ed.) Localities: The Changing Face of Urban Britain

London: Unwin Hyman

Burke, T. and Shackleton, J. R., (1996) Trouble in Store? UK Retailing in the 1990s Hobart Papers 130

London: Institute of Economic Affairs

Butler, R.W., (1980) The Concept of a tourism area cycle of evolution: implications for management of resources Canadian Geographer, Vol no. 24 (1), pp. 5-12

Butler, R.W., (2004) The tourism area life cycle in the twenty-first century In Lew, A.A., Hall, C.M. and Williams, A.M., (eds.) A Companion to Tourism, pp. 159-169

Oxford: Blackwell 
Butler, R.W., (ed.) (2005a) The Tourism Area Life Cycle Volume 1: Application and Modifications

Clevedon: Channel View Publications

Butler, R.W., (2005b) The Tourism Area Life Cycle: Volume 2: Conceptual and

Theoretical Issues

Clevedon: Channel View Publications

Butler, R.W., (2006a) The Concept of a Tourist Area Cycle of Evolution: Implications for Management of Resources In Butler R.W., (ed.) The Tourism Area Life Cycle, Vol no. 1, pp. 3-12

Clevedon: Channel View Publications

Butler, R.W., (2006b) The Origins of the Tourism Area Life Cycle In Butler R.W., (ed.)

The Tourism Area Life Cycle, Vol no. 1, pp. 13 - 26

Clevedon: Channel View Publications

Butler, S., (1914) Evolution, Old and New; Or, the Theories of Buffon, Dr. Erasmus Darwin and Lamarck, as compared with that of Charles Darwin

New York: E.P. Dutton \& Co

Burrell, G. and Morgan, G., (1979) Sociological Paradigms and Organisational Analysis In Cohen, L. and Manion, L., (2004) Research Methods in Education ( $4^{\text {th }}$ ed.)

London and New York: Routledge

Burton, J., (1992) Retail Rents

London: Adam Smith Institute

Byrne, D., (1998) Complexity Theory and the Social Sciences. An Introduction London: Routledge

CABE: Commission for Architecture and the Built Environment/English Heritage., (2003) Shifting Sands - Design and the changing image of English Seaside Towns Wolsey Press Limited

CABE: Commission for Architecture and the Built Environment (2010) Supermarkets the new drivers of urban growth Retrieved from

http://www.cabe.org.uk/articles/supermarkets

Cameron, D., (2009, November 10) The Big Society Hugo Young Lecture Retrieved from

www.conservatives.com/News/Speeches/2009/11/David_Cameron_The_Big_Society.as $\mathrm{px}$

Cameron, S., (2003) Gentrification, Housing Redifferentiation and Urban Regeneration: Going for Growth Newcastle-upon-Tyne Urban Studies Vol no. 40, pp. 2367-2382

Cannadine, D., (1982) Patricians, Politics and Power in $19^{\text {th }}$ Century Towns Leicester: Leicester University Press

Caro, R.G., Morris, R. and Norton, J.R., (2000) Advancing Aging Policy as the $21^{\text {st }}$ Century Begins Co-published simultaneously as Journal of Aging and Social Policy, Vol no. 11 , Numbers $2 / 3$

The Haworth Press

Carter, S.M. and Little, M., (2007) Justifying Knowledge, Justifying Method, Taking

Action: Epistemologies, Methodologies and Methods in Qualitative Research

Qualitative Health Research December 2007, Vol no. 17, 10, pp. 1316-1328

Casado-Diaz, M.A., Kaiser, C. and Warnes, A.M., (2004) Northern European retired residents in nine southern European areas: characteristics, motivations and adjustment Ageing and Society, Vol no. 24, pp. 353-381 
Cetron, M.J. and Davies, O., (2010) Trends Shaping Tomorrow's World Economic and Social Trends and Their Impacts

Bethesda USA: World Future Society

Champion, A.G., (1989) Counterurbanisation: The Changing Pace and Nature of Population Deconcentration

London: Edward Arnold

Champion, A.G., (2001) Urbanisation, Suburbanisation, Counterurbanisation and Reurbanisation In Paddison, R., (ed.) Handbook of Urban Studies, pp. 143-159 London: Sage Publications Ltd

Champion, A.G., (2005) Population movement within the UK In Chappell, R. (ed.) Focus on People and Migration, pp. 92-114

Basingstoke: Palgrave MacMillan

Chao, L., (2005) For Gen Xers, It's Work to Live - Allowing Employees to Strike Balance Between Job and Life Can Lead To Better Retention Rates

The Wall Street Journal, Online. 29 November 2005, pp. B6

Chaney, D., (1994) The Cultural Turn

London: Routledge

Charmaz, K., (2000) Grounded Theory: Objectivist and Constructivist Methods In Denzin, N.K. and Lincoln, Y.S. ( $2^{\text {nd }}$ ed.) Handbook of Qualitative Research

Thousand Oaks, California: Sage

CIPFA: Chartered Institute of Public Finance and Accountability, (2009) Value for Money (VfM) Reviews Retrieved from

www.cipfastats.net/

CIPFA/Solace: Chartered Institute of Public Finance and Accountancy/Society of Local Authority Chief Executives, (2009) After the downturn-Managing a significant and sustained adjustment in public sector funding Retrieved from http://www.cipfa.org.uk/PT/cipfasolace/download/After_the_Downturn.pdf

Church, A., Gilchrist, P., Heys, B., Palmer, C., Ravenscroft, N., Rogers, G., Sharp, G. and Watson, J., (2007) Tourism, Culture and Creativity: Preparing Hastings and Bexhill for Economic Regeneration through Education and Workforce Development in the Creative and Cultural Sectors of the Local Economy

Brighton: University of Brighton

Church, A. and Coles, T.E., (eds.) (2007) Tourism, Power and Space London: Routledge

Clandinin, D.J., (2007) Handbook of Narrative Inquiry: Mapping and Methodology Thousand Oakes, London, New Delhi: Sage

Clandinin, D.J. and Connelly, M., (2000) Narrative Inquiry: Experience and Story in Qualitative Research

San Francisco: Jossey-Bass

Clandinin, D.J. and Huber, J., (2010) Narrative inquiry In McGaw, B., Baker E. and Peterson P.P., (eds.), International Encyclopedia of Education ( $3^{\text {rd }}$ ed.)

New York, NY: Elsevier

Clarke, D.B., (1998) Consumption, identity and space-time Consumption, Markets and Culture, Vol no. 2 (3), pp. 233-258

Clarke, D. B., (2003) The Consumer Society and Postmodern City

London: Routledge 
Clegg, A. and Essex, S., (2000) Restructuring in tourism: The accommodation sector in a major British coastal resort International Journal of Tourism Research, Vol no. 2(2), pp. 77-95

Cohen, L. and Manion, L., (2004) Research Methods in Education (4 ${ }^{\text {th }}$ ed.) London and New York: Routledge

Coles, T.E., (2006) Enigma Variations? The TALC, marketing models and the descendents of the Product Life Cycle In Butler, R.W., (ed.) The Tourist Area Life Cycle: Conceptual and Theoretical Issues, pp. 49-66

Clevedon: Channel View Books

Coles, T.E., (2008) Telling tales of tourism: mobility, media and citizenship in the 2004 EU Enlargement In Burns, P.M. and Novelli, M., (eds.) Tourism and Mobility: LocalGlobal Connections, pp. 65-80

Wallingford: $C A B$ International

Coles, T.E., Duval, D.T. and Hall, C.M., (2004) Tourism, mobility and global communities: new approaches to theorising tourism and tourist spaces In Theobald, W., (ed.) Global Tourism, pp. 463-481

Amsterdam: Elsevier Butterworth-Heinemann

Coles, T.E. and Hall, C.M., (2006) The Geography of Tourism is Dead. Long Live Geographies of Tourism and Mobility

Current Issues in Tourism, Vol no. 9 (4/5), pp. 289-292

Coles, T.E. and Hall, C.M., (2008) International Business and Tourism: Global Issues, Contemporary Interactions London: Routledge

Coles, T.E. and Shaw G., (2006) Tourism property and the management of change in coastal resorts: Perspectives from southwest England

Current Issues in Tourism, Vol no. 9 (1), pp. 46-68

Coles, T.E. and Timothy D.J., (eds.) (2004) Tourism, Diasporas and Space London: Routledge

Conservative Party, (2010) Open Source Planning Green Paper Policy Green Paper No.14

Cooke, P., (1989) Locality, Economic Restructuring and World Development In Cooke, P., (ed.) Localities: The Changing Face of Urban Britain, pp. 1-44

London: Unwin Hyman

Cooper, C., (1992) The Lifecycles Concept and Strategic Planning for Coastal Resorts Built Environment, Vol no. 18 (1)

Cooper, C., (1997) Parameters and Indicators of the Decline of the British Seaside Resort In Shaw, G. and Williams, A.M., (eds.) The Rise and Fall of British Coastal Resorts, pp. 79-101

London: Cassell

Cooper, C., (2006) The Anatomy of the Rejuvenation Stage of the TALC In Butler R.W., (ed.) The Tourism Area Life Cycle, Vol no. 2, pp. 183-200

Clevedon: Channel View Publications

Cooper, C., Fletcher, J., Gilbert, D., Wanhill, S. and Shepherd, R., (1998) Tourism Principles and Practice $\left(2^{\text {nd }}\right.$ ed.)

New York: Addison Wesley Longman Publishing

Cooper, C. and Hall C.M., (2013) Contemporary Tourism: An International Approach ( $2^{\text {nd }}$ ed.)

Oxford: Goodfellow Publishers Ltd 
Cortazzi, M., (2001) Narrative Analysis in Ethnography In Atkinson, P., Coffey, A., Delamont, S., Lofland J. and Lofland.L., (eds.) Handbook of Ethnography, pp. 384-94 London: Sage

Couch, C., Fraser, C. and Percy, S., (2003) Urban Regeneration in Europe

Oxford: Blackwell

Coupland, A. (1997) Reclaiming the City: Mixed Use Development

Oxford: Chapman and Hall

Cox, J., Thurstain-Goodwin, M. and Tomalin, C., (2000) Town Centre Vitality and

Viability: A review of the Health Check Methodology Pilot Study A report for the National

Retail Planning Forum

London: Centre for Advanced Spatial Analysis, University College

Creswell, J.W., (2007) Research Design: Qualitative, Quantitative and Mixed Methods

Approaches $\left(2^{\text {nd }}\right.$ ed. $)$

Thousand Oakes, California: Sage

Creswell, J.W. and Plano, C.V.L., (2007) Designing and Conducting Mixed Methods

Research

Thousand Oaks, California: Sage

Crewe, L., (2000) Geographies of retailing and consumption Progress in Human

Geography, Vol no. 24 (2), pp. 275-290

Cross, D.F.W., (1990) Counterurbanization in England and Wales

Aldershot: Gower Publishing Ltd

Crotty, M., (2003) The Foundations of Social Research. Meaning and Perspective in the

Research Process

London: Sage

Croucher, K., (2006) Making the Case for Retirement Villages

York: Joseph Rowntree Foundation

Cuba, L.J. and Hammond, D.M., (1993) A place to call home and identification with dwelling, community and religion Sociological Quarterly, Vol no. 34, pp. 111-31

Danaher, G., Schirato, T. and Webb, J., (2000) Understanding Foulcault London: Sage

Davidson, M., (2010) Social Sustainability and the City

Geography Compass, Vol no. 4 (7), pp. 872-880

Davidson, R., (1989) Tourism

London: Pitman Publishing

Dawkins, R., (1986) The Blind Watchmaker

Penguin Books: London

Dawkins, R., (1989) The Selfish Gene (2 ${ }^{\text {nd }}$ ed.)

Oxford University Press: New York

De La Warr Pavilion, (2007) Economic Impact Evaluation Produced by Sussex Arts Marketing (SAM) and the University of Brighton

Dear, M., (2000) The Postmodern Urban Condition

Oxford: Blackwell

Dearlove, J. and Saunders, P., (2000) Introduction to British Politics ( $3^{\text {rd }}$ ed.)

Cambridge: Polity Press 
Deloitte and Oxford Economics, (2010) The Economic Contribution of the Visitor Economy: UK and the Nations

VisitBritain

Delsen, L. and Reday-Mulvey, G., (1996) Gradual Retirement in the OECD countries Aldershot, UK: Dartmouth

Demetriadi, J., (1995) English and Welsh Seaside Resorts: with Special Reference to Blackpool and Margate 1950 - 1974 Unpublished PHD Thesis, University of Lancaster, In Shaw, G. and Williams, A., (eds.) The Rise and Fall of British Resorts London: Pinter

Dennett, A. and Stillwell, J., (2009) Internal Migration in Britain, 2000-01, Examined Through an Area of Classification Framework Population, Space and Place Retrieved from

http://www3.interscience.wiley.com.ezproxy.brighton.ac.uk/cgi-bin/fulltext/ 122373228/PDFSTART?CRETRY $=1 \& S R E T R Y=0$

Denzin, N.K. and Lincoln, Y.S., (2000) Handbook of Qualitative Research ( $2^{\text {nd }}$ ed.) Thousand Oaks, California: Sage

Denzin, N.K. and Lincoln, Y.S., (2005) Handbook of Qualitative Research (3 ${ }^{\text {rd }}$ ed.) London: Sage

Denzin N.K. and Lincoln, Y.S., (2008) Strategies of Qualitative Inquiry ( $3^{\text {rd }}$ ed.) London: Sage Publications

DCLG: Department for Communities and Local Government, (2006a) State of the English Cities. A Comparison of Public Attitudes in Urban and Non-urban Areas across Different Regions Retrieved from London: Communities and Local Government publications http://www.communities.gov.uk/documents/regeneration/pdf/153104.pdf

DCLG: Department for Communities and Local Government, (2006b) State of the English Cities. The Changing Urban Scene: Demographics and the Big Picture Retrieved from London: Communities and Local Government publications http://www.communities.gov.uk/documents/regeneration/pdf/152672.pdf

DCLG: Department for Communities and Local Government, (2006c) The Dynamics of Local Economies and Deprived Neighbourhoods Retrieved from London: Communities and Local Government publications http://www.communities.gov.uk/publications/communities/economies

DCLG: Department for Communities and Local Government, (2007a) Extent of retail development taking place in England Retrieved from London: Communities and Local Government publications http://www.communities.gov.uk/publications/corporate/statistics/retaildevelopment2006

DCLG: Department for Communities and Local Government, (2007b) Indices of Deprivation Retrieved from London: Communities and Local Government publications www.communities/neighbourhoodrenewal/deprivation/deprivation07/

DCLG: Department for Communities and Local Government, (2007c) Committee on Coastal Towns: The Government's Second Response First Report of Session 20072008 (HC69)

London: The Stationery Office 2007

DCLG: Department for Communities and Local Government, (2007d) Government Response to the Communities and Local Government Committee Report on Coastal Towns (May 2007) (CM7126) 
London: The Stationery Office

DCLG: Department for Communities and Local Government, (2008a) The New Place Survey - Summary of responses to consultation

http://www.communities.gov.uk/publications/localgovernment/placesurveyresponses

DCLG: Department for Communities and Local Government, (2008b) Transforming

place; changing lives. A framework for Regeneration Retrieved from

London: Communities and Local Government Publications

http://www.communities.gov.uk/documents/regeneration/pdf/1189506.pdf

DCLG: Department for Communities and Local Government, (2009a) Attitudes to

Housing Findings from Ipsos MORI Public Affairs Monitor Omnibus Survey (England)

Retrieved from

London: Communities and Local Government Publications

http://www.communities.gov.uk/documents/housing/pdf/1298556.pdf

DCLG: Department of Communities and Local Government, (2009b) The Economic

Case for Cohesion Retrieved from

London: Communities and Local Government Publications

http://www.communities.gov.uk/documents/communities/pdf/1303560.pdf

DCLG: Department for Communities and Local Government, (2009c) Looking after our town centres Retrieved from

London: Communities and Local Government Publications

http://www.communities.gov.uk/documents/planningandbuilding/pdf/1201258.pdf

DCLG: Department for Communities and Local Government, (2009d) Housing and planning delivery grant (HPDG): Consultation on allocation mechanism for year 2 and year 3 Retrieved from

London: Communities and Local Government Publications

http://www.communities.gov.uk/documents/planningandbuilding/pdf/consultationdelivery grant.pdf

DCLG: Department for Communities and Local Government, (2009e) Place Survey

2008-2009 Retrieved from

London: Communities and Local Government Publications

http://www.communities.gov.uk/publications/localgovernment/placesurveyresponses

DCLG: Department for Communities and Local Government (2010a) Councils and

Business pick-up local Enterprise partnerships baton

https://www.gov.uk/government/news/councils-and-business-pick-up-local-enterprise-

partnerships-baton

DCLG Department for Communities and Local Government, (2010b) Strategy for

Seaside Success: Securing the future of seaside economies Retrieved from

London: Communities and Local Government Publications

http://www.communities.gov.uk/documents/citiesandregions/pdf/1518282.pdf

DCLG: Department for Communities and Local Government, (2010c) Letter from

Secretary of State for Communities and Local Government - Abolition of Regional

Strategies Retrieved from

http://www.communities.gov.uk/documents/planningandbuilding/pdf/1631904.pdf

DCLG: Department for Communities and Local Government, (2010d) Councils and Businesses to Rebalance Economy Retrieved from

www.communities.gov.uk/newsstories/newsroom/1626460

DCLG: Department for Communities and Local Government, (2010e) Functional

Economic Market Areas: An Economic Note Retrieved from

London: Communities and Local Government Publications.

http://www.communities.gov.uk/documents/localgovernment/pdf/1469713.pdf 
DCLG: Department for Communities and Local Government, (2010f) Local Government Finance Settlement 2011/2012 Retrieved from http://www.local.communities.gov.uk/finance/1112/grant.htm

DCLG: Department for Communities and Local Government, (2010g) Evaluation of the National Strategy for Neighbourhood Renewal: Local Research Project Retrieved from http://www.communities.gov.uk/documents/communities/pdf/1490532.pdf

DCLG: Department for Communities and Local Government, (2010h) English House Condition Survey- Headline Report 2008-2009 23 ${ }^{\text {rd }}$ February 2010 Retrieved from http://www.communities.gov.uk/publications/corporate/statistics/ehs200809headlinerepo $\mathrm{rt}$

DCMS: Department of Culture, Media and Sport, (2004) Culture at the Heart of Regeneration

London: Department of Culture Media and Sport publishing Retrieved from http://webarchive.nationalarchives.gov.uk/+/http://NR/rdonlyres/e2pbs42ddx3nsmm4ovh 6eva5mv4jfb7fglcrez6ybcxf2u2czr5rvovcrud2socwizbgxxft6xt3j7pwt22kjpn6jga/DCMSC ulture.pdf

Department of Health, (2007) Putting People First. A shared vision and commitment to the transformation of Adult Social Care Retrieved from http://www.dh.gov.uk/en/Publicationsandstatistics/Publications/PublicationsPolicyAndGu idance/DH_081118

Department for Transport, (2012) Announcement of Bexhill to Hastings Link Road Retrieved from http://www.dft.gov.uk/publications/bexhill-to-hastings-link-road/

De Roo, G. and Miller, D., (eds.) (2004) Integrating City Planning and Environmental Improvement: Practical Strategies for Sustainable Urban Development

Aldershot: Ashgate

Dey, I., (1993) Qualitative Data Analysis

London: Routledge

Directgov, (2010) Getting a State Pension Forecast Retrieved from

http://www.direct.gov.uk/en/Pensionandretirementplanning/StatePension/StatePensionfo recast/DG_10014008

DTZ/Pieda, (2006) Housing Market Assessment of Hastings and Rother - Final Report Hastings Borough Council and Rother District Council

Reading: DTZ/Pieda Consulting

DTZ, (2010) Hastings and Rother Strategic Housing Market Assessment - Update 2009/2010 Hastings Borough Council and Rother District Council London: DTZ

Dunnell, K., (2001) Policy Responses to population ageing and population decline in the United Kingdom Population Trends, Vol no.103, pp. 47-52

DWP: Department for Work and Pensions, (2001) Incapacity Benefits and Work Incentives Research Report No. 141 - Corden, A. and Sainsbury, R. A report of research carried out by the Social Policy Research Unit on behalf of the Department for Work and Pensions Retrieved from

http://research.dwp.gov.uk/asd/asd5/rrep141.pdf

DWP: Department for Work and Pensions, (2003) Full Employment in Every Region London: H.M. Treasury http://www.dwp.gov.uk/docs/5yr-annex1.pdf

DWP: Department for Work and Pensions, (2005) A Social Portrait of Ageing in the UK London: Department for Work and Pensions publishing 
http://www.dwp.gov.uk/publications/publications-archive/policy-and-strategy-archive/

DWP: Department for Work and Pensions, (2006) Security in retirement: towards a new pension system

London: Department for Work and Pensions publishing

http://www.dwp.gov.uk/docs/white-paper-complete.pdf

DWP: Department for Work and Pensions, (2009a) Family Resources Survey United Kingdom 2007-2008

London: Department for Work and Pensions publishing

http://research.dwp.gov.uk/asd/frs/2008_09/frs_2008_09_report.pdf

DWP: Department of Work and Pensions, (2009b) A social portrait of ageing in the UK London: Department for Work and Pensions publishing

http://www.dwp.gov.uk/publications/publications-archive/policy-and-strategy-archive/

DWP: Department for Work and Pensions, (2010a) Incapacity Benefits -Reassessing

Claims Retrieved from

http://www.dwp.gov.uk/adviser/updates/ib-reassessing-claims/

DWP: Department for Work and Pensions, (2010b) Employment and Support Allowance ESA14 - The Work Capability Assessment

http://research.dwp.gov.uk/asd/workingage/esa_wca/esa_wca_27072010.pdf

Dwyer, C. and Limb, M., (2001) Introduction; Doing Qualitative Research in Geography In Limb and Dwyer, (eds.) Qualitative Methodologies for Geographers. Issues and Debates, pp. 1-20

London: Arnold

Elliott, J., (1997) Tourism, Politics and public sector management London: Routledge

ELSA: English Longitudinal Study of Ageing, (2003) Marmot, M., Banks, J., Blundell, R., Lessof, C. and Nazroo, J., (eds.) Health, wealth and lifestyles of the older population in England: The 2002 English Longitudinal Study of Ageing (Wave 1)

London: The Institute for Fiscal Studies

ELSA: English Longitudinal Study of Ageing, (2006) Banks. J., Breeze, E., Lessof, C. and Nazroo, J., (eds.) Retirement, Health and Relationships of the Older Population in England: The 2004 English Longitudinal Study of Ageing (Wave 2)

London: The Institute for Fiscal Studies

ELSA: English Longitudinal Study of Ageing, (2008) Banks, J., Breeze, E., Lessof, C. and Nazroo, J., (eds.) Living in the 21 $1^{\text {st }}$ century: older people in England: The 2006 English Longitudinal Study of Ageing (Wave 3)

London: The Institute for Fiscal Studies

ELSA: English Longitudinal Study of Ageing, (2010) (Wave 4) Banks, J., Breeze, E., Lessof, C. and Nazroo, J., (eds.) Financial circumstances, health and well-being of the older population in England: The 2008 English Longitudinal Study of Ageing (Wave 4) London: The Institute for Fiscal Studies.

English Heritage, (2007) Regeneration in Historic Coastal Towns Retrieved from London: English Heritage publishing

http://www.helm.org.uk/guidance-library/regeneration-historic-coastal-towns/coastalregeneration.pdf

English Heritage and Urban Practitioners, (2007) An Asset and a Challenge; Heritage and Regeneration in Coastal Towns in England

London: Urban Practitioners

English Tourist Board, (1991) The Future of England's Smaller Seaside Resorts London: English Tourist Board 
English Tourism Council, (2001) Sea Changes: Creating World Class resorts in England: A Strategy for Regenerating England's Resorts

London: English Tourism Council publishing

Erikson, E., (1987) The Life Cycle Completed Extended version with new chapters on the Ninth Stage of Development, Erikson J. M.

London and New York: W.W. Norton \& Company

ESCC: East Sussex County Council, (1958) East Sussex County Development Plan Lewes: County Hall Archives

ESCC: East Sussex County Council, (2008) East Sussex Economic Study 2008

Lewes: County Hall Archives

ESCC: East Sussex County Council, (2009) Bexhill and Hastings Link Road Newsletter No. 1Retrieved from

http://www.eastsussex.gov.uk/NR/rdonlyres/FDD5BB36-FFBD-4C8B-BC26-

75D363F7C989/0/bhlr_newsletter_sep_09.pdf

ESCC: East Sussex County Council, (2010a) Bexhill and Hastings Link Road Newsletter No. 2 Retrieved from

http://www.eastsussex.gov.uk/NR/rdonlyres/1D6FD404-BD4A-4465-99A1-

4AEBA1778C69/0/bhlr_newsletter_feb_10.pdf

ESif: East Sussex County Council East Sussex in Figures, (2010b) Dependency Ratios Retrieved from http://www.eastsussexinfigures.org.uk/webview/index.jsp?stubs=Geography\&study=http $\% 3 \mathrm{~A} \% 2 \mathrm{~F} \% 2 \mathrm{Fesfigures} 01 \mathrm{~s}$.escc.gov.uk\%3A80\%2Fobj\%2FfStudy\%2F317\&virtualslice= Number_value\&Geographyslice $=21 \mathrm{UC} \&$ measuretype $=4$ \&headers $=$ Year\&headers $=$ Num bersandratios \&mode $=$ cube $\&$ Yearslice $=2026 \& v=2 \&$ Numbersandratiosslice $=$ Ratio $+1+\& c u$ be=http\%3A\%2F\%2Fesfigures01s.escc.gov.uk\%3A80\%2Fobj\%2FfCube\%2F317_C1\&Y earsubset $=2006 \% 2 \mathrm{C} 2026 \&$ Geographysubset $=21 \% 2 \mathrm{C} 21 \mathrm{UC}+-$

$+21 \mathrm{UH} \&$ layers $=$ virtual\&top $=$ yes

ESif: East Sussex County Council East Sussex in Figures, (2010c) Population estimates and demographic trends Retrieved from

http://www.eastsussexinfigures.org.uk

ESif: East Sussex County Council East Sussex in Figures, (2012) Indices of Multiple Deprivations - Super Output Areas for Bexhill-on-Sea Retrieved from www.eastsussexinfigures.org.uk

ESRC: Economic \& Social Research Council (2012) ESRC Seminar: Rethinking Retirement

Seminar 4: Activity, Unpaid Work and Active Ageing

University of Brighton: University of Edinburgh: University of Kent

Ettlinger, N., (2009) Problematizing the presentation of poststructural case-study research, or working out the crisis of representation in the presentation of empirics Environment and Planning, Vol no. 41 (5), pp. 1017-1019

European Policies Research Centre, (2006) Regions for All Ages: The Implications of Demographic Ageing for Regional Policy Final Report prepared by the European Policies Research Centre, University of Strathclyde to the Regions for All Ages Research Programme (supported by the European Older People's Platform (AGE) Age Concern England, Northern Ireland Executive Office, North West Regional Assembly and Scottish Enterprise

Evandrou, M., (1996) Baby Boomers: Ageing in the $21^{\text {st }}$ Century London: Age Concern

Evandrou, M. and Falkingham, J., (2006) Will the Baby Boomers be better off than their 
Parents in Retirement In Vincent, J., Phillipson, C. and Downs M., (2006) The Futures of Old Age

London: Sage Publications

Evandrou, M., Falkingham, J. and Green, M., (2010) Migration in Later Life: Evidence from the British Household Panel Study

ESRC Centre for Population Change and Centre for Research on Ageing: University of Southampton

Evans, G., (2009a) Creative Cities, Creative Spaces and Urban Policy Urban Studies, Vol no. $46(5 / 6)$, pp. 1003-1040

Evans, G., (2009b) Accessibility, Urban Design and the Whole Journey Environment Built Environment, Vol no. 35 (3) Retrieved from

http://www.citiesinstitute.org/londonmet/fms/MRSite/Research/cities/Built\%20Env.pdf

Evans, G., (2009c) From cultural quarters to creative clusters - creative spaces in the new city economy In Leger, M., (ed.) The sustainability and development of cultural quarters: international perspectives

Stockholm: Institute of Urban History

Evans, G. and Foord, J., (2009) Urban sustainability: mixed use and mixed messages In Cooper, R., Evans, G.L. and Boyko, C., (eds.) Designing Sustainable Cities, 190-217

Oxford: Blackwell-Wiley

Evans, G. and Shaw P., (2004) The Contribution of Culture to Regeneration in the UK: a Review of Evidence A report to the Department for Culture Media and Sport London Metropolitan University

Fairley, A., (2001) Bucking the Trend: The Life and Times of the Ninth Earl De La Warr $1900-1976$

Bexhill-on-Sea: The Pavilion Trust

Fairley, A., (2006) The De La Warr Pavilion, the modernist masterpiece London: Merrell Publishers Ltd

Featherstone, M., (1991) Consumer Culture and Postmodernism London: Sage

Feldman, M.W. and Laland, K.N., (1996) Gene-culture coevolutionary theory Trends in Ecology and Evolution 11, pp. 453-57

Fielding, A.J., (1988) Inter-regional migration and social change: a study of South East England based upon data from the Longitudinal Study In Robinson, V., (1996)

Geography and Migration

Cheltenham: Edward Elgar Publishing Ltd

Fielding, A.J., (1992) Migration and social mobility: South East England as an escalator region Regional Studies, Vol no. 16, pp. 1-15

Findlay, A., Short, D. and Stockdale, A., (1999) Migration Impacts in Rural England Cheltenham: Countryside Agency

Flick, U., (2006) An Introduction to Qualitative Research (3 $3^{\text {rd }}$ ed.)

London: Sage

Flick, U., (2007) Designing Qualitative Research

London: Sage

Flowerdew, R. and Martin, D., (2005) Methods in Human Geography A guide for students doing a research project

England: Pearson Prentice Hall 
Florida, R., (2004) The Rise of the Creative Class: and how its transforming work, leisure, community and everyday life

New York: Basic Books

Florida, R., (2005) Cities and the Creative Class

London: Routledge

Florida, R., (2007) The Flight of the Creative Class: the global competition for talent New York: Collins

Florida, R., (2008) Who's Your City? How the Creative Economy in Making Where to Live the Most Important Decision of your Life

New York: Basic Books

Florida, R., (2010) The Great Reset - How New Ways of Living and Working Drive PostCrash Prosperity

New York: HarperCollins Publishers

Florida, R. and Mellander, C., (2007) There goes the neighbourhood: How and why Artists, Bohemians and Gays Affect Housing Values Retrieved from http://creativeclass.typepad.com/thecreativityexchange/files/Florida_Mellander_Housing _Values_1.pdf

Florida, R. and Timagli, I., (2004) Europe in the Creative Age

Pittsburg: Carnegie Mellan Software Industry Centre

Fokkema, T., Gierveld, J. and Nijkamp. P., (1996) Big Cities, Big problems: reasons for elderly to move?

Urban Studies: Urban Studies Journal Limited

Ford, H., (2008) Pathways to Management and Leadership $2^{\text {nd }}$ Edition

Chartered Management Institute

Foster, D.M. and Murphy, P. (1991) Resort cycle revisited - The retirement connection Annals of Tourism Research 18, $553-567$

Fothergill, S., (2007) England's Coastal Towns, A short review of the issues, evidence base and research needs for NCRA Panel CRESR, Sheffield Hallam University

Retrieved from

https://www.ljmu.ac.uk/EIUA/EIUA_Docs/6_Fothergill.pdf

Fothergill, S., (2012) The Coastal Communities of South East England:

Recommendations to the South East LEP Final Report - December 2012

Centre for Regional Economic and Social Research: Sheffield Hallam University

Fotheringham, A.S., Rees, P., Champion, T., Kalogirou, S. and Tremayne, A.R., (2004) The development of a migration model for England and Wales: overview and modelling out-migration Environment and Planning, Vol no. 36, pp. 1633-1672

Foulcault, M., (1986) Of Other Spaces

Diacritics, Vol no. 16, pp. 22-27

Frey, W.H., (1999) Beyond social security: The local aspects of an aging America Washington, DC: The Brookings Institution

Frey, W.H. and Devol, R.C., (2000) America's Demography in the New Century: Ageing Baby Boomers and New Immigrants as Major Players Milken Institute Policy Brief: March

Gaffickin, F. and Warf, B., (1993) Urban policy and the post-Keynesian state in the United Kingdom and the United States International Journal of Urban and Regional Research, Vol no. 17, pp. 67-84 
Gale, T., (2005) Modernism, post-modernism and the decline of British seaside resorts as long holiday destinations: A case study of Rhyl, North Wales Tourism Geographies, Vol no. 7 (1), pp. 86-112

Garcia, B., (2004) Urban Regeneration, Arts Programming and Major Events' Glasgow 1990, Sydney 2000 and Barcelona 2004

International Journal of Cultural Policy, Vol no. 10 (1), pp. 103-118

Garner, R. and Manning, K., (2010) A Rubicon for the Public Sector? An Analysis of the Coalition's programme for public services Tribal Group Retrieved from www.tribalgroup.com

Garrett, A., (2008, March 9) Trouble up north as apartment prices slump Retrieved from http://www.guardian.co.uk/money/2008/mar/09/houseprices.moneyobserver

Gaventa, S., (2009) Poised on the Brink - Action Planning Event (follow up to Wavelength Conference 17 October 2008, Hastings) Commission for Architecture and the Built Environment

Giddens, A., (1991) Modernity and self-identity: self and society in the late modern age Cambridge: Polity Press

Gilbert, E.W., (1954) Brighton Old Oceans Bauble

London: Harvester (reprinted Hassocks, 1976)

Glaser, K. and Grundy, E., (2002) Class, Caring and Disability: Evidence from the British Retirement Survey Ageing and Society, Vol no. 22 (3), pp. 325

Glasgow, N., (1990) Attracting Retirees as a Community Development Option Journal of the Community Development Society, Vol no. 21, pp. 102-114

Godfrey, M., Townsend, J. and Denby, T., (2004) Building a good life for older people in local communities

York: Joseph Rowntree Foundation

Goldman, N., Korenman, S. and Weinstein, R., (1995) Marital status and Health among the Elderly Social Science and Medicine, Vol no. 40, pp. 1717-1730

Goodson, L. and Phillimore, J., (2004) The inquiry paradigm in qualitative tourism research In Phillimore and Goodson, (eds.) Qualitative Research in Tourism:

Epistemology, Ontology and Methodology, pp. 30-45

London: Routledge

Gordon, I. and Goodall, B., (2000) Localities and Tourism Tourism Geographies, Vol no. 2 (3), pp. 290-311

GOSE: Government Office for the South East (2009) South East Plan: Regional Spatial Strategy for the South East of England TSO (Stationery Office) Retrieved from www.gos.gov.k/gose/planning/regionalplanning/815640

Gove, M.A. Rt Hon MP., (2012) Presentation to Bexhill High School on $7^{\text {th }}$ December 2012 Quote adapted from Winston Churchill speech to the House of Commons $28^{\text {th }}$ October 1944

Government Select Committee, (2009) Future Policy Thinking: What Future for England's Struggling Towns? Si Policy Roundtable Briefing Paper Retrieved from http://www.margaterenewal.co.uk/pdf/What\%20Future\%20Briefing\%20Paper.pdf

Gray, F., (1994) Bexhill Voices

Falmer: Centre for Continuing Education, University of Sussex.

Green, A., (2006) Employment and the Older Person in the Countryside In Lowe, P. and Speakman, L., (eds.) The Ageing Countryside: The Growing Older Population of Rural 
England

London: Age Concern England

Grigg, D.B., (1977) E.G. Ravenstein and the 'laws of migration' In Robinson, V., (ed.)

Geography and Migration

Cheltenham: Edward Elgar Publishing Ltd

Grimshaw, L. and Smith I., (2007) Evaluation, knowledge and learning in neighbourhood governance: the case of the New Deal for Communities In Smith, I., Lepine, E. and Taylor, M., (eds.) Disadvantaged By Where you Live? Neighbourhood Governance in Contemporary Urban Policy Bristol: Polity Press 185 - 204

Guardian, (2010, July 18) Britons renew their love of seaside resorts as cost of holidays abroad soars

Guba, E.G. and Lincoln, Y.S., (2004) Competing Paradigms in Qualitative Research:

Theories and Issues In Hesse-Biber and Nagy, (eds.) Approaches to Qualitative

Research: a reader on theory and practice

New York: Oxford University Press

Gubrium, J. and Holstein, J., (1997) The New Language of Qualitative Methods

New York: Oxford University Press

Guilmont, A., (1982) Bexhill-on-Sea: A Pictorial History

Chichester: Phillimore

Gunder, M. and Hillier, J., (2009) Planning in Ten Words or Less: A Lacanian

Entanglement with Spatial Planning

Aldershot: Ashgate

Gunter, B., (1998) Understanding the Older Consumer - the Grey Market

Routledge

Gurran, N., (2008) The Turning Tide: Amenity Migration in Coastal Australia International Planning Studies, Vol no. 13 (4), pp. 391-414

Gyourko, J.E. and Sinai, T.M., (2001) The Spatial Distribution of housing-related tax benefits in the United States National Bureau of Economic Research (NBER) Working Paper Series Cambridge, Mass: National Bureau of Economic Research, Vol 81652001

Gyourko, J.E. and Sinai, T.M., National Bureau of Economic Research (2006) Superstar Cities' Cambridge, Mass: National Bureau of Economic Research, Vol no. 1, pp. 23-55 July 2006

Haber, C. and Gratton, B., (1994) Old Age and the search for security: An American Social History Indiana: University Press

Halcrow Group, (2010) Spending Review 2010 Retrieved from http://halcrow.co.uk/News/2010-news-archive/

Hall, P., (1998) Cities in Civilization

London: Weidenfield and Nicolson

Hall, T., (1998) Urban Geography

London: Routledge

Hamilton, M.M., (2003, March 19) Embarking On a Second Act Retrieved from www.washingtonpost.com/ac2/wp-dyn?pagename=articlmode=\&content ID=A534

Hammersley, M. and Atkinson, P., (2007) Ethnography: Principles in Practice (3 ${ }^{\text {rd }}$ ed.) London: Tavistock 
Hamnett, C., (1991) The blind men and the elephant: the explanation of gentrification? Transactions of the Institute of British Geographers, Vol no. 16, pp. 173-189

Hamnett, C., (2003) Gentrification and the middle class remaking of Inner London: $1961-$ 2001 Urban Studies, Vol no. 40, pp. 2401-2426

Hamnett, C., (2008, March 5) Buy to Let-or to lose? The Guardian

Harvey, D., (2005) A Brief History of Neoliberalism

Oxford: Oxford University Press

HBC: Hastings Borough Council (2012) Council Tax Bands and Budgets Retrieved from http://www.hastings.gov.uk/housing_tax_benefits/council_tax/council_tax_values_charg es/

Hay, I., (2005) Qualitative Research Methods in Human Geography

Oxford: Oxford University Press

Hayden, C., Boaz, A. and Taylor, F., (1999) Attitudes and Aspirations of Older People: A qualitative study DSS Research Report No. 102, Leeds, CDS

Haywood, M.K., (2006a) Evolution of Tourism Areas and the Tourism Industry In Butler R.W., (ed.) The Tourism Area Life Cycle, Vol no. 1, pp. 51-70

Clevedon: Channel View Publications

Haywood, M.K., (2006b) Legitimising the TALC as a Theory of Development and Change In Butler R.W., (ed.) The Tourism Area Life Cycle, Vol no. 2, pp. 29-44 Clevedon: Channel View Publications

Healey, P., (2007) Urban Complexity and Spatial Strategies: Towards a Relational Planning for our Times

London: Routledge

Hearn Property Consultants, (2008) Rother District Wide Shopping Assessment Rother District Council

London: Hearn Property Consultants

Hearn Property Consultants, (2010) Testing of Retail Scenarios for Bexhill Rother District Council

London: Hearn Property Consultants

Hedges, A., (1998) Pensions and retirement planning DSS Research Report no 83, Corporate Document Services: Leeds Retrieved from http://research.dwp.gov.uk/asd/asd5/report_abstracts/rr_abstracts/rra_083.asp

Heet, J.A., (2003) Beyond Workforce 2020 The Coming (and Present) International Market for Labor Hudson Institute White Paper (October) www.hudson.org

Herbert D., (2000) Towns and Cities In Gardiner V. and Matthews H., (eds.) The Changing Geography of the United Kingdom ( $3^{\text {rd }}$ ed.), pp. $190-212$ London: Routledge

Heritage Lottery Fund, (2010) Investing in success Heritage and the UK Tourism Economy Retrieved from

http://www.hlf.org.uk/news/Pages/InvestinginSuccess.aspx

Hernández, J.M., Hernández, L. and Carmelo, C.J., (2007) Analysis: The interactions between natural and physical capitals in the tourist lifecycle model ScienceDirect www.sciencedirect.com

Hetherington, K., (1998) Expressions of Identity: Space, Performance, Politics London: Sage 
Hewlett, S.A., Sherbin, L and Sumberg, K., (2009) How Gen Y \& Boomers Will Reshape Your Agenda Harvard Business Review July-August 2009 Retrieved from http://www.talkpoint.com.au/talkpoint/filelibrary/files/Resources/2010/How_GenY_and_B oomers_will_reshape_your_agenda.pdf

Higginbottom, K., (2005) Seaside Towns Invest for Future Prosperity

Estates Gazette - Issue

Hirsch, D., (2003) Crossroads after 50

York: Joseph Rowntree Foundation Retrieved from

http://www.jrf.org.uk/sites/files/jrf/1859351565.pdf

HM Government, (2009a) Jobs of the Future

(CM7654) London: HM Treasury

HM Government, (2009b) PSA Delivery Agreement 17: Tackle Poverty and Promote Greater Independence and Wellbeing in Later Life

(PU373) London: HM Treasury

HM Treasury, (2009) Putting the Frontline First: Smarter government

London

(CM7753) London: HM Treasury

HM Treasury: Her Majesty's Treasury, (2010) Spending Review 2010

(CM7942) London: HM Treasury

Hochschild, A., (1983) The Managed Heart: Commercialisation of Human Feeling

Berkeley: University of California press

Hodgson, G.M., (2001) Is Social Evolution Lamarckian or Darwinian? In Laurent J. and Nightingale J. (eds.) Darwinism and Evolutionary Economics, pp. 87-118

Cheltenham: Edward Elgar

Hodgson, G.M., (2005) Generalizing Darwinism to Social Evolution: Some Early

Attempts

Journal of Economic Issues, Vol XXXIX No. $4^{\text {th }}$ December 2005

Hodgson, G.M. and Knudsen, T., (2006) Dismantling Lamarckism: why descriptions of socio-economic evolution as Lamarkian are misleading Journal of Evolutionary

Economics, Vol no. 16, pp. 343-366

Holstein, J. and Gubrium, J., (2008) Handbook of Constructionist Research

New York: Guildford

Horner, P., (1991) The Travel Industry in Britain

Cheltenham: Dept of Employment

Horrigan, M.W., (2004) Employment Projections to 2012: Concepts and Context Monthly Labor Review Retrieved from

http://www.bls.gov/opub/mlr/2004/02/art1full.pdf

Huberman, A.M. and Miles, M.B., (1994) Data Management and Analysis Methods In Denzin and Lincoln, (eds.) Handbook of Qualitative Research

Thousand Oaks, California: Sage

Hyde, K.F., Ryan, C. and Woodside, A.G., (2012) Field Guide to Case Study Research

in Tourism, Hospitality and Leisure

Tourism and Hospitality series 6

Ibarra, P.R. and Kusenbach, M., (2001) Feeling the field: Tracking shifts in ethnographic research

Studies in Symbolic Interaction, Vol no. 24, pp. 195-221 
Imrie, R. and Mike, R., (2004) Urban Renaissance? New Labour, Community and Urban Policy

The Policy Press: Bristol

Independent, (2012, October 2) Over the Hill? Come to Bexhill where life begins at 100 Retrieved from

http://www.independent.co.uk/news/uk/this-britain/over-the-hill-come-to-bexhill-wherelife-begins-at-100-8193198.html

Inside Housing, (2010, October 22) Councils to move LHA claimants out of London Retrieved from http://www.insidehousing.co.uk/news/housing-management/councils-to-move-lhaclaimants-out-of-london/6512167.article

Ipsos MORI., (2009, March 30) Results of the Place Survey for Rother District Council and partners Presentation by Harkness, V. and Collins, B., Ipsos MORI to Rother District Council Retrieved from

http://www.rother.gov.uk/media/pdf/q/0/Rother_District_Council_2008_Place_Survey_Pr esentation_FINAL_for_PDF_Read-Only_.pdf

Jacobs, J., (1993) The Death and Life of Great American Cities (ed.)

New York: Random House

Janesick, V.J., (1998) The Dance of Qualitative Research: Metaphore, Methodolatry and Meaning In Denzin and Lincoln (eds.) Strategies of Qualitative Inquiry, pp. 35-55 London: Sage

Jasper, M., (2003) Beginning Reflective Practice: Foundations in nursing and health care

Cheltenham: Nelson Thornes

Jayne, M., (2006) Cities and Consumption

Routledge: Oxon

Jefferies, J., (2005) The UK Population: past, present and future

Focus On: People and Migration Retrieved from

http://www.ons.gov.uk/ons/rel/fertility-analysis/focus-on-people-and-migration/december2005/focus-on-people-and-migration---focus-on-people-and-migration---chapter-1.pdf.

Johnson, M.L., (ed.) in association with Bergstson, V.L., Coleman, P.G. and Kirkwood, T.B.L., (2005) The Cambridge Handbook of Ageing Cambridge: Cambridge University Press also cited in Law, C.H. and Warnes, A.M.,1982 Destination Decision in Retirement Migration

Johnston, S., (2006) The Ontological Foundation of the TALC In Butler R.W., (ed.) The Tourism Area Life Cycle, Vol no. 2, pp. 7-28

Clevedon: Channel View Publications

Jowell, R., Witherspoon, S. and Brooks, L., (1997) British Social Attitudes Survey, $14^{\text {th }}$ report: The end of conservative values?

Ashgate: Aldershot

Jonassen, D., (1991) Objectivism versus Constructivism: Do we need a new philosophical paradigm?

Journal of Educational Technology Research and Development, Vol no. 39 (3), pp. 5-14

Karn, V.A., (1977) Retiring to the Seaside

London and Boston: Routledge and K. Paul

Keat, R., Whitley, N. and Abercombie, N., (1994) The Authority of the Consumer

London: Routledge

Keyfitz, N., (1982) Can Knowledge Improve Forecasts? 
Population and Development Review, Vol. 8, No. 4 (Dec., 1982), pp. 729-751

King, A., (1984) The Bungalow: The production of a global culture

King, R., (2002) Towards a New Map of European Migration

International Journal of Population Geography, Vol no. 8, pp. 89-106

King, R., Warnes, A.M. and Williams, A.M., (1998) International Retirement Migration in Europe

International Journal of Population Geography, Vol no. 4, pp. 91-111

King, R., Warnes, A.M. and Williams, A.M., (2000) Sunset Lives: British Retirement to the Mediterranean Retrieved from

http://books.google.co.uk/books?hl=en\&lr=\&id=PB rVcR3PK4C\&oi=fnd\&pg=PR7\&dq=r etiring+to+the+seaside,+karn\&ots $=91$ XsO5OOrc\&sig=fwvrEavNxBXsNyLfur3PtqVA7Lk\# $\mathrm{v}=$ onepage $\& \mathrm{q}=$ retiring $\% 20$ to $\% 20$ the $\% 20$ seaside $\% 2 \mathrm{C} \% 20 \mathrm{karn} \& \mathrm{f}=$ false

King, V., (2010) The Generational Rivalry for Time Time and Society Retrieved from http://tas.sagepub.com/content/19/1/54.short

Korczyk, S.M., (2002) Back to Which Future: The U.S. Aging Crisis Revisited Washington: American Association for Retired Persons (AARP) Public Policy Institute Retrieved from http://assets.aarp.org/rgcenter/econ/2002_18_aging.pdf

Lagiewski, R,M., (2006) The Application of the TALC Model: A Literature Survey In Butler R.W., (ed.) The Tourism Area Life Cycle, Vol no. 1, pp. 27-50

Clevedon: Channel View Publications

Laland, K.N., Odling-Smee, J. and Feldman, M.W., (2000) Niche construction, biological evolution and cultural change

Behavioural and Brain Sciences, Vol no. 23, pp. 131-175

Lamarck, J.B., (1809) Philosophie zoologique Translated 1963 as Zoological

Philosophy: an exposition with regard to the natural history of animals

New York: Harner publishing Co

Lampedusa, G. T., (2007) The Leopard

Original title 'Il Gattopardo' $1^{\text {st }}$ published 1958 - pp. 29. English translation 1961

New York: Pantheon

Lancaster, L.C. and Stillman, D., (2003) When generations collide: Who they are: Why they clash: How to solve the generational puzzle at work

New York: HarperCollins

Lash, S. and Urry, J., (1994) Economies of Sign and Spaces

London: Sage

Law, C.M., (1998) Urban revitalisation, public policy and the redevelopment of redundant port zones: lessons from Baltimore and Manchester In Hoyle, B.S., Pinder, D.A. and Hussain, M.S., (eds.) Revitalising the Waterfront: International dimensions of Dockland redevelopment, pp. 146-166

London: Belhaven

Law, C.M. and Warnes, A.M., (1982) The destination decision in retirement migration In Warnes A.M., (ed.) Geographical Perspectives on the Elderly, pp. 478.

Chichester: Wiley

Le Mesurier, N., (2006) The Contributions of Older People to Rural Community and Citizenship In Lowe, P. and Speakman, L., (eds.) The Ageing Countryside: The Growing Older Population of Rural England

London: Age Concern England 
Lee, H. and Lee, J., (2008) Time to Change, Time for Change: Time as a catalyst for organizational change Time Society September 2008, Vol 17, no. 2-3, pp. 363-384

Lee, M.J. (1992) Consumer Culture Reborn: The Cultural Politics of Consumption London: Routledge

Lees, L., (2000) A reappraisal of gentrification: towards a geography of gentrification Progress in Human Geography, Vol no. 24, pp. 389-408

Lees, L., (2003a) Policy (re)turns: gentrification research and urban policy - urban policy and gentrification research Environment and Planning, Vol no. 35, pp. 571-574

Lees, L., (2003b) Visions of 'urban renaissance': the Urban Task Force report and the Urban White Paper In Imrie R. and Raco M., (eds.) Urban Renaissance? New Labour Community and Urban Policy, pp. 61-82

Bristol: Policy Press

Lees, L., (2008) Gentrification and social mixing towards an inclusive urban renaissance Urban Studies, Vol no. 45, pp. 2449-2470

Lees, L., Slater, T. and Wyly, E., (2008) Gentrification

London: Routledge

Lefebvre, H., (1991) The Production of Space

Oxford: Blackwell

Lever, S., (2007) Learn to like to be beside the seaside again

Caterer and Hotelkeeper $15^{\text {th }}$ March 2007, Vol 197

Ley, D., (1996) The New Middle Class and the Remaking of the Central City

Oxford: Oxford University Press

Lincolnshire Coastal Action Zone, (2006) National Coast Futures Report of Symposium at Royal Renaissance Hotel, Skegness 18/19 ${ }^{\text {th }}$ July 2006

Lindley, K., (1973) Seaside Architecture

London: Evelyn

Litwak, E. and Longino, C.F. Jr., (1987) Migration patterns among the elderly: a developmental perspective Gerontologist, Vol no. 27 (3), pp. 266-72

Lofland, J. and Lofland, L., (1995) Analysing Social Settings: A Guide to Qualitative Observation and Analysis ( $3^{\text {rd }}$ ed.)

Belmont, California: Wadsworth

Longino, C.F. Jr. and Warnes, A.M., (2005) Migration and Older People In Johnson, M.L., (ed.) The Cambridge Handbook of Ageing, pp. 538-544

Cambridge University Press

McCarthy, J. (1995). The Dundee Waterfront. A missed opportunity for planned regeneration Land Use Policy, Vol no. 12 (4), pp. 307-319

McCarthy, J., (1998) Reconstruction, Regeneration and Re-imaging

Cities, Vol no.15 (5), pp. 337-344

Macleod, D. and Ward, K., (2002) Spaces of Utopia and Dystopia: the contemporary city Geografiska Annaler, Vol no. 84, pp. 153-170 In Tallon, A., (ed.) Urban Regeneration in the UK

Oxford: Routledge

Maffesoli, M., (1996) The Time of the Tribes: The Decline of Individualism in Mass

Society

London: Sage 
Margate Renewal Partnership, (2008) Margate Renewal Study (2007/08) Retrieved from

http://www.margaterenewal.co.uk/pdf/Margate\%20Renewal\%20Study.pd

Mark, J., (2007) Cities and Consumption

Oxon: Routledge

Marsden, G., (2003) The last resort? Locum Destination Review, Vol no. 13, pp. 54-57

Marshall, H. and Williamson, H., (eds.), (1994) Law and Valuation of Leisure Property London: Estates Gazette

Martin, B., (2006) The TALC Model and Politics In Butler, R.W., (ed.) The Tourism Area Life Cycle, Vol 1, pp. 237-249

Clevedon: Channel View Publications

Martin, R. and Sunley, P., (2007) Complexity Thinking and Evolutionary Economic Geography Journal of Economic Geography, Vol no. 7 (5), pp. 573-601

Mason, J., (2002) Qualitative Researching $2^{\text {nd }}$ ed.

London: Thousand Oakes: New Delhi: Sage

May, T., (2001) Social research: issues, methods and process

Buckingham: Open University Press

Mayr, E., (1982) The Growth of Biological Thought: Diversity, Evolution and Inheritance Cambridge, MA: Harvard University Press

McGill, I., Brockbank, A. and Beech, N., (2002) Reflective Learning in Practice Aldershot: Gower

McHugh, K.E., (1990) Seasonal migration as a substitute for, or precursor to, permanent migration Research on Aging, Vol no. 12, pp. 229-45

McHugh, K.E., (2003) Three faces of ageism, society, image and place Ageing and Society, Vol no. 23 (2), pp. 165-86

Means, R., (1996) Home, independence and community care: time for a wider vision Policy and Politics, Vol no. 25 (4)

Mehmood, A., (2010) On the History and Potentials of Evolutionary Metaphors in Urban Planning Planning Theory 2010, Vol no. 9, pp. 63-87

Metcalfe, J.S., (1993) Some Lamarckian Themes in the Theory of Growth and Economic Selection: A Provisional Analysis

Revue Internationale De Systemique, Vol no. 7, pp. 487-504

Midgley, G., Munlo, I. and Brown, M., (1997) Sharing Power - integrating user involvement and multi-agency working to improve housing for older people Policy Press: Bristol

Midwinter, E., (1991) The British Gas Report on Attitudes to Ageing London: British Gas

Miles, M., (2005) Interruptions: Testing the Rhetoric of Culturally Led Urban Development Urban Studies, Vol no. 42 (5)

Miles, M.B. and Huberman, A.M., (1984) Qualitative Data Analysis: An Expanded Sourcebook

Beverley Hills, California: Sage

Miles, M., Tim, H. and Borden, I., (2004) The City Cultures Reader ( $2^{\text {nd }}$ ed.) 
Oxon: Routledge

Miles, S., (2001) Social Theory in the Real World

London: Sage

Miles, S. and Miles, M., (2004) Consuming Cities

Hampshire: Palgrave Macmillan

Mineau, G.P., Smith, K.R. and Bean, L.L., (2002) Historical trends of survival among widows and widowers Social Science and Medicine, Vol no. 54, pp. 245-254

Moen, P., (2003) Midcourse: Reconfiguring careers and community service for a new life stage Contemporary Gerontology, Vol no. 9 (3), pp. 1-8

Moen, P., (2005) Beyond the Career Mystique: "Time In" and "Time Out" and "Second Acts" Sociological Forum, Vol no. 20 (2), pp. 189-208

http://www.jstor.org/stable/4540892

Montgomery, J., (1995) Urban Vitality and the Culture of Cities In Planning Practice and Research, Vol no. 10, pp. 101-109

Morris, J., (1976) (ed.) Domesday Book, Sussex

Chichester: Phillimore, 1976

Morgan, N., (1992) Perceptions, Patterns, Policies of Tourism: the Development of the Devon Seaside Resorts during the $20^{\text {th }}$ Century - with special reference to Torquay and Ilfracombe Unpublished PHD Thesis at University of Exeter In Shaw, G. and Williams, A.M., (eds.) 1997 The Rise and Fall of British Coastal Resorts London: Pinter

Moser, C.A. and Scott, W., (1961) British Towns

London: Oxford University Press

Mueller, S. and Thomas, A.S., (2000) Culture and entrepreneurial potential; A nine country study of locus of control and innovativeness Journal of Business Venturing, Vol no. 16 , pp. $51-75$

Murakami, K., Atterton, J. and Gilroy, R., (2008) Planning for the Ageing Countryside in Britain and Japan: City Regions and the Mobility of Older People Centre for Rural Economy - Newcastle University GURU - Global Urban Research Unit Retrieved from http://www.ncl.ac.uk/cre/research/ageing_countryside_UK_Japan.htm

Murdoch, J., (2006) Post-structuralist Geography

London: Sage

Nathan, M. and Urwin C., (2006) City People: City Centre Living in the UK London: Institute for Public Policy Research

NEF: New Economic Foundation, (2007) Final Evaluation of the Hastings and St Leonards SRB Programme Hastings: Hastings Borough Council

Newman, O., (1983) The coming of a leisure society? Leisure Studies, Vol no. 2, pp. 97109

Nickell, S. and Quintini, G., (2003) The recent performance of the UK labour market Oxford Review of Economic Policy, Vol no. 18, pp. 202-20

O'Connor, J. and Wynne D., (1993) From the margins to the centre: cultural production and consumption in the post-industrial city Working Papers in Popular Cultural Studies No. 7, Manchester Institute for Popular Culture

Oc, T. and Tiesdell, S., (1997) The death and life of city centres In Oc, T. and Tiesdell 
S., (eds.) Safer City Centres: Reviving the Public Realm, pp. 1-20

London: Paul Chapman Publishing Ltd

ODPM: Office of the Deputy Prime Minister, (2000) Our Towns and Cities - the Future The Urban White Paper Retrieved from

http://www.odpm.gov.uk/stellent/groups/odpm_urbanpolicy/documents/pdf/odpm_urbpol _pdf_608358.pdf

ODPM: Office of the Deputy Prime Minister, (2006a) State of the English Cities Volume 1

London: ODPM Publications

ODPM: Office of the Deputy Prime Minister, (2006b) State of the English Cities Volume 2

London: ODPM Publications

ODPM: Office of the Deputy Prime Minister, (2006c) A Sure Start to Later Life: Ending Inequalities for Older People A Social Exclusion Unit Final Report (January)

Oliver, C., (2007) Retirement Migration: Paradoxes of Ageing

London: Routledge

ONS: Office of National Statistics, (2003) Social Trends Summerfield, C. and Babb, P., No.33 Retrieved from

http://www.statistics.gov.uk/downloads/theme_social/social_trends37/social_trends_33. pdf

ONS: Office of National Statistics, (2006) Time Use Survey 2005 Lader, D., Short, S. and Gershuny, J. A report on research using the ONS Omnibus Survey produced on behalf of the Economic and Social Research Council, Department of Culture, Media and Sport, Department for Education and Skills, Department of Health, Department for Transport, Office for National Statistics (July) Retrieved from http://www.statistics.gov.uk/articles/nojournal/time_use_2005.pdf

ONS: Office of National Statistics, (2007) Social Trends Self, A. and Zealey, L. No. 37 Retrieved from

http://www.statistics.gov.uk/downloads/theme_social/social_trends37/social_trends_37. pdf

ONS: Office of National Statistics, (2008) Social Trends Self, A. and Zealey, L. No. 38 Retrieved from

http://www.statistics.gov.uk/downloads/theme_social/Social_Trends38/Social_Trends_3 8.pdf

ONS: Office of National Statistics, (2009) Family Spending: A report on the 2008 Living Costs and Food Survey Skentelberg, R.

Palgrave, MacMillan Retrieved from www.statistics.gov.uk/downloads/theme_social/family-spending-2008/ familyspending2009.pdf

ONS: Office of National Statistics, (2010a) Social Trends Self, A., Hughes, M. and Church, J. No. 40 Retrieved from

http://www.statistics.gov.uk/downloads/theme_social/Social-Trends40/

ST40_2010_FINAL.pdf

ONS: Office of National Statistics, (2010b) Life Expectancy at Birth, UK, from period life tables, 1980-1982 to 2007-2009 Retrieved from

http://www.statistics.gov.uk/cci/nugget.asp?ID=168

ONS: Office of National Statistics (2010c) Life Expectancy in the UK: 1991-2009 by

Local Authority Area Retrieved from

http://www.statistics.gov.uk/life-expectancy/lifemap.html 
ONS: Office of National Statistics (2010d) 2009 Mid-year Estimates Retrieved from http://www.statistics.gov.uk/statbase/Product.asp?vink=15106

ONS: Office of National Statistics (2012, February 10) Indices of Multiple Deprivation Neighbourhood Statistics Retrieved from http://www.neighbourhood.statistics.gov.uk

Oppenheim, A., (1992) Questionnaire design, interviewing and attitude measurement London: Pinter

O'Reilly, K., (2000) The British on the Costa del Sol: Transnational Identities and Local Communities

London: Routledge

O'Reilly, K., (2003) When is a tourist? The Articulation of Tourism and Migration in Spain's Costa del Sol Tourist Studies, Vol no. 3 (3), pp. 301-317

O'Reilly, K., (2007a) The Rural Idyll, Residential Tourism, and the Spirit of Lifestyle Migration Paper presented at the ASA (Association of Social Anthropologists) 2007 Thinking Through Tourism

O'Reilly, K., (2007b) Intra-European Migration and the mobility-enclosure dialectic Sociology, Vol no. 41 (2), pp. 277-293

O'Reilly, K., (2007c) Emerging tourism futures: residential tourism and its implications In Geoffroy, C. and Sibley, R., (eds.) Going Abroad: Travel, Tourism, and Migration. CrossCultural Perspectives on Mobility. Newcastle upon Tyne, pp. 144-157

Cambridge Scholars Publishing

Packard, A.S., (2007) Lamarck, the Founder of Evolution New York: Longmans \& Green Co

Pacione, M., (1997) Britain's Cities: Geographies of Division in Urban Britain London: Routledge

Pacione, M., (2005) Urban Geography: A Global Perspective London: Routledge $\left(2^{\text {nd }}\right.$ ed. $)$

Page, S. and Hall, C.M., (2003) Managing Urban Tourism London: Prentice Hall

Palmer, J.P., (2002) Bread and Circuses: The Local Benefits of Sports and Cultural Businesses

Ottawa, Ontario: C.D. Howe Institute

Papatheodorou, A., (2004) Exploring the Evolution of Tourism Resorts Annals of Tourism Research, Vol no. 31 (1), pp. 219-237

Parry, J., Vegeris, S., Hudson, M., Barnes, H. and Taylor, R., (2004) Independent living in later life Research Report No. 216 by the Policy Studies Institute on behalf of the Department for Work and Pensions Retrieved from http://research.dwp.gov.uk/asd/asd5/rports2003-2004/rrep216.pdf

Patton, M.Q., (2002) Qualitative research and evaluation methods ( $3^{\text {rd }}$ ed.) Thousand Oaks, California: Sage

Pearce, P.L., Moscardo, G. and Ross, G.F., (1996) Tourism Community Relationships Oxford: Pergamon Press

Peck, J., (2005) Struggling with the Creative Class International Journal of Urban and Regional Research, Vol no. 29 (4), pp. 740-770 
Peet, R., (1998) Modern geography Thought

Oxford: Blackwell

Pender, L. and Sharpley, R., (2005) The Management of Tourism

London: Sage

Pensions Commission, (2004) Pensions: Challenges and Choices - The First Report of the Pensions Commission Retrieved from

http://image.guardian.co.uk/sys-files/Money/documents/ 2005/05/17/ fullreport.pdf

Perkin, H., (1976) The "social tone" of Victorian seaside resorts in the north-west Northern History, X1:180-94 In Urry, J., 1997 Consuming Places

Perrons, D., Fagan, C., McDowell, L., Ray, K. and Ward, K., (2005) Work, Life and Time in the New Economy: An introduction Time and Society, Vol no. 14 (1), pp. 51-64 London: Sage Publications

Peterson, R.T., (2000) Small Retailer Hiring of Older Workers: An Assessment Academy of Entrepreneurship Journal of Business Strategies 2007, Vol no. 6.1, pp. 18-57

Phillips, B., (1998) Over the Hill \& on a Roll: Laugh lines for the better half of life Oregon: Harvest House Publishers

Phillips, B.D., (2004) The Future Small Business Workforce: Will Labor Shortages Exist? The Available Evidence is less than perfect The Journal of National Association of Business Economics, Vol. XXXIX October 2004 No.4

Phillips, M., (2002) The production, symbolization and socialization of gentrification: Impressions from two Berkshire villages Transactions of the Institute of British Geographers, Vol. 27 (3), pp. 282-308

Plog, S., (1974) Why Destination Areas Rise and Fall in Popularity Cornell Hotel, Restaurant and Administration Quarterly, Vol no. 14 (4), pp. 55-58

Polhemus, T., (1995) Streetstyle: From Sidewalk to Catwalk

London: Thames and Hudson

Polkinghorne, D., (1988) Narrative Knowing in Human Sciences

Albany: State University of New York Press

Por, F.D., (2006) The Actuality of Lamarck: towards the bicentenary of his Philosophie Zoologique Integrative Zoology 2006; 1: pp. 48-52

Blackwells Publishing: IOZ and CAS

Porter J., (2004) Bexhill-on-Sea, A History

Chichester: Phillimore and Co. Ltd

Porter, L. and Barber, A., (2006) The Meaning of Place and State-led Gentrification in Birmingham's Eastside City: analysis of urban trends, culture, theory, policy, action, Vol no. 10 (2), pp. 215-234

Potter, J., (1997) Discourse Analysis as a Way of Analysing Naturally Occurring Talk In Silverman D., (ed.) Qualitative Research: Theory, Method and Practice London: Sage

Potter, S., (2006) Doing Postgraduate Research, $\left(2^{\text {nd }}\right.$ ed.)

London, Thousand Oakes, New Delhi: Sage

Powe, N.A. and Hart, T., (2009) Competing for the custom of small town residents: exploring the challenges and potential International Journal of Retail and Distribution Management, Vol no. 37 (9), pp. 732-747

Powe, N.A. and Shaw, T., (2004) Exploring the current and future role of market towns 
in servicing their hinterlands: a case study of Alnwick in the North East of England Journal of Rural Studies, ISSN: 0743-0167, 20 (4), October, pp. 405-418

PriceWaterhouseCoopers, (2010a) Capable Communities: Public Service reform: The next chapter Retrieved from

http://www.ippr.org/publications/capable-communities-public-service-reform-the-next-

chapter

PriceWaterhouseCoopers, (2010b) Towards a smarter state: $A$ joint programme from ippr and PriceWaterhouseCoopers Retrieved from

http://www.pwc.co.uk/pdf/towards_a_smarter_state.pdf

Purdue, D., Razzaque, K., Hambleton, R., Stewart, M., Huxham, C. and Vangen, S., (2000) Community Leadership in Area Regeneration

Bristol: Policy Press

Ravenscroft, N., (1999) The Vitality and Viability of Town Centres Urban Studies, 37:13, pp. 2533-2549

Ravenscroft, N. and Gilchrist, P., (2009) The Emergent Working Society of Leisure Journal of Leisure Research, Vol no. 41 (1), pp. 23-39

Ravenscroft, N. and Hadjihambi I., (2006) The Implications of Lamarckian Theory for the Tourism Area Life Cycle Model In Butler, R.W., (ed.) The Tourism Area Life Cycle:

Conceptual and Theoretical Issues, Vol no. 2, pp. 150-163

Ravenscroft, N., Reeves, J. and Rowley, M., (2000) Leisure, property, and the viability of town centres Environment and Planning, Vol no. 32 (8), pp. 1359-1374

Rees, P., Stillwell, J., Convey, A. and Kupiszewski, M., (1996) Population Migration in the European Union

Chichester: John Wiley and Sons Ltd

RDA: Regional Development Agencies (2010) Regional Development Agencies Abolished Retrieved from www.englandsrdas.com/news/government-seeks-lep-proposals-to-repl ace-rdas

RDC: Rother District Council, Cabinet Minutes 2004-2010 Retrieved from Internal Archives

Bexhill: Rother District Council

RDC: Rother District Council, (2003) Transfer of De La Warr Pavilion to the De La Warr Pavilion Charitable Trust Ltd Retrieved from

Archives - CB19/02/03

Bexhill: Rother District Council

RDC: Rother District Council, (2006a) Rother in Profile Bexhill: Rother District Council October 2006

Bexhill: Rother District Council Retrieved from

http://www.rother.gov.uk/media/pdf/q/a/Rother_in_Profile.pdf

RDC: Rother District Council, (2006b) Rother District Council Corporate Plan (20062016)

Bexhill: Rother District Council Retrieved from

http://www.rother.gov.uk/media/pdf/n/k/Corporate_Plan_2007_(Yellowduck).pdf

RDC: Rother District Council (2006c) Rother District Local Plan

Bexhill: Rother District Council Retrieved from

http://www.rother.gov.uk/media/pdf/c/s/local-plan_1.pdf

RDC: Rother District Council, (2007a) Delivering Housing Solutions: A Housing Strategy for Rother, 2007-2012 Rother District Council housing department

Bexhill: Rother District Council Retrieved from 
http://www.rother.gov.uk/media/pdf/m/r/housing_strategy_2007-2012.pdf

RDC: Rother District Council, (2007b) Investment Opportunities in Bexhill-on-Sea

Retrieved from

http://www.rother.gov.uk/CHttpHandler.ashx?id=4349\&p=0

Bexhill: Rother District Council

RDC: Rother District Council, (2008) Local Development Framework Core Strategy,

Consultation on Strategy Directions

Bexhill: Rother District Council Retrieved from

http://www.rother.gov.uk/media/pdf/i/i/Core_Strategy_'Strategy_Directions'_FINAL_PDF 1.pdf

RDC: Rother District Council, (2010) Rother District Affordable Housing Viability Assessment A report prepared by DTZ/Pieda for Rother District Council London: DTZ/Pieda

RDC: Rother District Council, Cabinet Minutes 2011-2013

Bexhill: Rother District Council Retrieved from

http://www.rother.gov.uk/article/306/Cabinet

RDC: Rother District Council, (2011a) Revenue Budget and Capital Programme Monitoring 2007/08 - 2011/12 Retrieved from

Archives - C07/64/03/08

Archives - C08/52/03/09

Archives - C09/59/03/10

Archives - C10/64/02/11

Bexhill: Rother District Council

RDC: Rother District Council, (2011b) Rother Economic Regeneration Strategy 2010/2015

Bexhill: Rother District Council Retrieved from

http://www.rother.gov.uk/media/pdf/6/h/Rothers_Economic_Regeneration_Strategy_201

0_2015.pdf

RDC: Rother District Council (2011c) Local Development Framework Core Strategy,

Proposed Submission Core Strategy

Bexhill: Rother District Council Retrieved from

http://www.rother.gov.uk/media/pdf/2/o/Proposed_Submission_Core_Strategy.pdf

RDC: Rother District Council (2011d) Hastings and Rother Employment Strategy and

Land Review Update

Bexhill: Rother District Council Retrieved from

http://www.rother.gov.uk/media/pdf/b/t/Hastings_and_Rother_Employment_Strategy_Re

view_Update.pdf

RDC Rother District Council (2011e) Bexhill Town Centre Steering Group Minutes-

Town Centre Shop Occupancy

Bexhill: Rother District Council Retrieved from

www.rother.gov.uk/article/8116/bexhill-town-centre-steering-group-meeting-1st-

september-2011

RDC: Rother District Council (2012) Council Tax Bands and Budgets

Bexhill: Rother District Council Retrieved from

http://www.rother.gov.uk/counciltax

Rensma, R., (2013) Analytical Psychology and the Ghost of Lamarck Journal of Analytical Psychology 58 (1), pp. 258-277

Rentfrow, P.J., Gosling, S.D. and Potter J., (2008) The Geography of Personality: A Theory of the Emergence, Persistence and Expression of Geographic Variation in

Psychological Characteristics Perspectives on Psychological Science (September ) 
Richards, G., (2007) Cultural Tourism: Global and Local Perspectives

Binghampton, New York: Haworth Press

Roberts., M., (2007) Sharing space: urban design and social mixing in mixed income new communities Planning Theory and Practice, Vol no. 8, pp. 183-204

Robinson, V., (1996) Geography and Migration

Cheltenham: Edward Elgar Publishing Ltd

Robson, B.T., Bradford, M. and Deas, I., (1994) Assessing the Impact of Urban Policy London: HMSO

Rodriguez, V., Fernandez-Mayoralas, G. and Rojo, F., (1998) European Retirees on the Costa del Sol: A Cross-National Comparison International Journal of Population

Geography, Vol no. 4 (2), pp. 91-111

Rojek, C., (1990) Ways of Escape

London: Macmillan

Rojek, C., (2000) Leisure and the rich today: Veblen's thesis after a century

Nottingham: Nottingham Trent University Faculty of Humanities

Rojek, C., (2001) Leisure and life politics Leisure Sciences, Vol no. 23, pp. 115-125

Rosenau, P.M., (1992) Post Modernism and Social Sciences: Insights, Inroads and Intrusions

Princetown: Princetown University Press

Rother Conservatives, (2011) Rother Conservatives Party Manifesto

Promoted by Gillian Wheeler

Uckfield: Chris Dowling

Rother Voluntary Action, (2010) Rother Local Action Plans Support Project

Bexhill: Rother District Council Archieves

Rowles, G.D. and Watkins, J.F., (2001) Elderly Migration and Development in Small Communities Growth and Change, Vol no. 24, pp. 509-538

Rugg, G. and Petre, M., (2007) A Gentle Guide to Research Methods New York: Open University Press

Russell, R. and Faulkner, B., (2004) Entrepreneurship, Chaos and the Tourism Area Lifecycle Annals of Tourism Research, Vol no. 31 (3), pp. 556-579

Russell, R., (2006a) The Contribution of Entrepreneurship Theory to the TALC Model In Butler R.W., (ed.) The Tourism Area Life Cycle, Vol no. 2, pp. 105-123

Russell, R., (2006b) Chaos Theory and its Application to the TALC Model In Butler R.W., (ed.) The Tourism Area Life Cycle, Vol no. 2, pp. 164-180

Ryder, T., (2005) Sea change's still got it Money, November 2005 Issue 10, pp. 64-67

Safer Rother Partnership, (2010) Community Safety in your Area Survey 2009 East Sussex County Council Community Safety Analysts Retrieved from http://www.rother.gov.uk/index.cfm?articleid=354

Sainsbury's, (2009) Meeting in London $6^{\text {th }}$ October 2009 and $14^{\text {th }}$ January 2010 Borkowski, C. Chairman and CEO of St. James Investments; Webster, Q. Development Executive St. James Investments; Webster, T. Consultant to St. James Investments; Thompson, H. Development Surveyor for Sainsbury's; Chalkley, T. Grant Mills Wood External Development for Sainsbury's; Davidson, M. White Young Green Planning Agents for Sainsbury's 
Sander, N., Skirbekk, V., Samir, K.C. and Lundevaller, E., (2010) Prospects for Later-life Migration in Urban Europe PLUREL report: Peri-urban land use relationships strategies and sustainability assessment tools for urban-rural linkages, integrated project (October)

Sarup, M., (1993) An Introductory Guide to Post-Structuralism and Postmodernism Harlow: Pearson Education Limited

Saunders, M.N.K., Lewes, P. and Thornhill, A., (2000) Research Methods for Business Students $\left(4^{\text {th }}\right.$ ed.)

Harlow: Prentice Hall

Scharf, T., Phillipson, C. and Smith, A.E., (2002) Growing Older in socially deprived areas: Social Exclusion in later Life

London: Help the Aged

Schulz, J.H., (1992) The Economics of Aging ( $5^{\text {th }}$ ed.)

New York: Auburn House

Scott, A.J., (2000) The Cultural Economy of Cities: Essays on the Geographies of Image Producing Industries

London: Sage

Scott, J., (2000) Is it a different world to when you were growing up? Generational effects on social representations and child-rearing values British Journal of Sociology, Vol no. 51, pp. 355-376

Searson, D., (1998) Raj Connections with Bexhill and East Sussex

Bexhill: Searson

Seaspace: Hastings and Bexhill Renaissance Ltd. (2003a) The Business Plan for Coastal Regeneration in Hastings and Bexhill Hastings and Bexhill Taskforce DTZ Peida Consulting Retrieved from

http://www.seaspace.org.uk/community/regeneration-plan/

Seaspace: Hastings and Bexhill Renaissance Ltd. (2003b) Hastings and Bexhill

Taskforce Five Point Plan Hastings and Bexhill Taskforce - DTZ Peida Consultancy

Retrieved from

www.seaspace.org.uk/community/regeneration-plan/

Seaspace: Hastings and Bexhill Renaissance Ltd. (2010a) Innovation Centre Retrieved from

www.innovationcentrehastings.com/

Seaspace: Hastings and Bexhill Renaissance Ltd. (2010b) (confidential papers) Special Board Meeting $15^{\text {th }}$ November 2010 Sale of One Priory Square to SAGA

Secker, J., Hill, R., Villeneau, L. and Parkman, S., (2003) Promoting Independence: But Promoting What and How? Ageing and Society, 23(3): pp. 375

SEEDA: South East England Development Agency, (2008) SEEDA Evaluation report on Hastings and Bexhill Renaissance Limited Retrieved from http://www.seeda.co.uk/_publications/Sea_Space_Evaluation_Report250908.pdf

Shackleton, R., (2004) The Retirement Prospects of Baby Boomers Congressional Budget Office Economic and Budget Issue Brief $18^{\text {th }}$ March 2004 Retrieved from http://www.cbo.gov/doc.cfm?index $=5195 \&$ type $=0$

Shaw, G. and Agarwal, S., (2007) Introduction. The Development and Management of Coastal Resorts: A Global Perspective In Agarwal, S. and Shaw, G., (eds.) Managing Coastal Resorts: A Global Perspective, pp. 1-18

Clevedon: Channel View Publications 
Shaw, G. and Coles, T., (2007) The Resort Economy: Changing Structures and Management Issues in British Resorts In Agarwal, S. and Shaw, G., (eds.) Managing Coastal Resorts: A Global Perspective, pp. 40-55

Clevedon: Channel View Publications

Shaw, G. and Williams, A., (1997) The Rise and Fall of British Coastal Resorts London: Pinter

Shaw, G. and Williams, A.M., (2002) Critical Issues in Tourism: A Geographical Perspective ( $2^{\text {nd }}$ ed.)

Oxford: Blackwell

Shaw, G. and Williams, A.M., (2004) Tourism and Tourism Spaces

London: Sage

Sheller, M. and Urry, J., (2004) Tourism Mobilities: Places to play, places in play London: Routledge

SHRM: Society of Human Research Management, (2004) Generational differences survey report A study by Alexandria, V.A. for the Society for Human Resources Management

Silverman, D., (2001) Interpreting Qualitative Data - Methods for Analysing Talk, Text and Interaction ( $2^{\text {nd }}$ ed.)

London: Sage Publications

Silverman, D., (2005) Doing Qualitative Research. A Practical Handbook (2 ${ }^{\text {nd }}$ ed.) London: Sage

Silverman, D., (2006) Interpreting Qualitative Data (3 ${ }^{\text {rd }}$ ed.)

London: Sage

Silverman, D., (2009) Doing Qualitative Research

London: Sage Publications

Silverman, D., (2010) Qualitative Research (3 ${ }^{\text {rd }}$ ed.)

London: Sage Publications

Simms, A., Oram, J., MacGillivray, A. and Drury, J., (2002) Ghost Town Britain: The Threat from Economic Globalisation to Livelihoods, Liberty and Local Economic Freedom

London: New Economics Foundation

Simms, A., Kjell, P. and Potts, R., (2005) Clone Town Britain: The Survey Results on the Bland State of the Nation

London: New Economics Foundation

Slater, T., (2006) The eviction of critical perspectives from gentrification research International Journal of Urban and Regional Research, Vol no. 30, pp. 737-757

Smith-Conway, K. and Houtenville, A.J., (2003) Out with the Old, In with the Old - A closer look at Younger versus Older Elderly Migration Cornell University Social Science Quarterly, Southwestern Social Science Association, Vol no. 84 (2)

Smith, A., (2007) After the Circus Leaves Town: the Relationship between Sport Events, Tourism and Urban Regeneration In Smith, M.K., (ed.) Tourism, Culture and

Regeneration, pp. 85-100

Wallingford: $\mathrm{CABI}$

Smith D., (2002) Patterns and processes of 'studentification' in Leeds The Regional Review, Vol no. 12, pp. 15-16

Smith, D.P. and Denholm, J., (2006) Studentification: A Guide to Challenges, 
Opportunities and Practice

London: Universities UK

Smith, J.K., (1983) Quantitative versus Qualitative Research: An Attempt to Clarify the Issue

Educational Researcher, Vol no. 12 (3), pp. 6-13

Smith, K.R. and Zick, C.D., (1994) Do close parent-child relations reduce the mortality of older parents? Journal and Health and Social Behaviour 32: pp. 382-395

Smith, M.J., (1998) Social Science in question

London: Sage

Smith, M.K., (2003) Issues in Cultural Tourism Studies

London: Routledge

Smith, M.K., (2004) Seeing a New Side to Seaside's: Culturally Regenerating the English Seaside Town

International Journal of Tourism Research, Vol no. 6 (1), pp. 17-28

Smith, M.K., (2007) Towards a Cultural Planning Approach to Regeneration In Smith M.K., (ed.) Tourism, Culture and Regeneration, pp. 1-11

Wallingford: $\mathrm{CABI}$

Smith, N., (1986) Gentrification, the frontier, and the restructuring of urban space In Smith, N. and Williams, P., (eds.) Gentrification of the City, pp. 15-34

Boston, MA: Allen \& Unwin

Smith, N., (1987) Gentrification and the Rent Gap Annuals of the Association of America Geographers Sept 1987, Vol no. 77 (3), pp. 462-78

Smith, N., (1996) The New Urban Frontier: Gentrification and the Revanchist City London: Routledge

Smith, N., (2000) Gentrification In Johnston, R.J. Gregory, D., Pratt G. and Waitts M., ( $4^{\text {th }}$ ed.) The Dictionary of Human Geography pp. 295-296

Oxford: Blackwell

Smith, N., (2002) New globalism, new urbanism: gentrification as global urban strategy Antipode, Vol no. 34, pp. 428-450

Smola, K.W. and Sutton, C.D., (2002) Generational differences: Revisiting generational work values for the new millennium Journal of Organisational Behaviour, Vol no. 23, pp. 363-382

Soja, E., (1996) Thirdspace: Journey to Los Angeles and Other Real-and-Imagined

Places

Oxford: Blackwell

Soule, A., Babb, P., Evandrou, M., Balchin, S. and Zealey, L., (2005) Focus on Older People Department for Work and Pensions

DWP: Palgrave Macmillan

South East Regional Assembly, (2004) Sussex Coastal Towns Sub-Regional Study Retrieved from http://www.eastsussex.gov.uk/NR/rdonlyres/A6577F81-3E4E-4490-B102-C1B3E71A-

709C/0/FinalReportAppendices30Apr04.pdf

Stafford, F. and Yates, N., (1985) The Later Kentish Seaside

Gloucester: Allan Sutton

Stake, R.E., (1995) The art of case study research

Thousand Oaks, California: Sage 
Stake, R.E., (2000) Case Studies In Denzin N.K. and Lincoln Y.S., (eds.) Handbook of Qualitative Research, pp. 435-454

Stake, R.E., (2005) Qualitative case studies In Denzin N.K. and Lincoln Y.S. The Sage handbook of qualitative research $\left(3^{\text {rd }}\right.$ ed.) pp. 443-466

Thousand Oaks, California: Sage

Stake: R.E., (2006) Multiple case study analysis

New York: Guilford

Stallibrass, C., (1980) Seaside Resorts and the Hotel Accommodation Industry Progress in Planning, Vol no. 13 (3)

Stead, D. and Hoppenbrouwer, E., (2004) Promoting an Urban Renaissance in England and the Netherlands Cities, Vol no. 21 (2), pp. 119-136

Steel, M. and Symes, M., (2005) The privatisation of public space? The American experience of Business Improvement Districts and their relationship to local governance Local Government Studies 31: pp. 321-334

Steinnes, D.N. and Hogan T.D., (1992) Take the money and the sun: elderly migration as a consequence of gains in unaffordable housing markets Journal of Gerontology Social Sciences, Vol no. 47 (4), pp. 197-203

Stevens, R. and Willis, P., (1990) Earl De La Warr and the Competition for Bexhill Pavilion 1933-34

Cardiff: SAHGB Publications Ltd

Stevenson, D., (2003) Cities and Urban Cultures London: Sage

Stoecker, R., (1991) Evaluating and rethinking the case study The Sociological Review, Vol no. 39, pp. 88-112

Stone, I. and Stubbs, C., (2007) Enterprising expatriates: lifestyle migration and entrepreneurship in rural southern Europe Entrepreneurship \& Regional Development 19(5) pp. 433-450.

Strauss, A. and Corbin, J.M., (1998) Basics of Qualitative Research: Techniques and Procedures for Developing Ground Theory

Thousand Oaks, California: Sage

Strauss, A. and Corbin, J.M., (2008) Basis of Qualitative Research ( $3^{\text {rd }}$ ed.) London: Sage

Sullivan, O., (2008) Busyness, Status Distinction and Consumption Strategies of the Income Rich, Time Poor, Time and Society Retrieved from http://tas.sagepub.com/content/17/1/5

Sunil, T., Rojas, V. and Bradley, D., (2007) United States' international retirement migration: the reasons for retiring to the environs of Lake Chapala, Mexico Ageing and Society, Vol no. 27, pp. 489-510

Swarbrooke, J., (2000) Tourism, economic development and urban regeneration: $A$ critical evaluation In Robinson, M., Sharpley, R., Evans, N., Long, P., Swarbrooke, J., (eds.) Developments in Urban and Rural Tourism

Sunderland: Business Education Publishers

Sweetman, P., (1999) Anchoring the (Postmodern) Self? Body Modification, Fashion and Identity Body and Society, Vol no. 5 (2-3), pp. 51-76 
Sweetman, P., (2003) Twenty-first century dis-ease? Habitual reflexivity or the reflexive habitus The Sociological Review, Vol no. 51(4), pp. 528-549

Tallon, A.R., (2007) Bristol: City Profile Cities, Vol no. 24 (1), pp. 74-88

Tallon, A.R., (2010) Urban Regeneration in the UK

Oxford: Routledge

Tallon, A.R. and Bromley, R.D.F., (2004) Exploring the attractions of city centre living evidence and policy implications in British cities Geoforum, Vol no. 35, pp. 771-787

Tallon, A.R., Bromley, R.D.F. and Thomas, C.J., (2005) Swansea: City Profile Cities, Vol no. 22 (1), pp. 65-76

Taylor, M., (2000) Communities in the lead: power, organisational capacity and social capital Urban Studies, Vol no. 37, pp. 1019-1035

Taylor, M., (2003) Public Policy in the Community

Basingstoke: Palgrave Macmillan

Taylor, M., (2007) Community participation in the real world: opportunities and pitfalls in new governance spaces Urban Studies, Vol no. 44, pp. 291-317

Taylor, S., (2001) Locating and Conducting Discourse Analytic Research In Wetherell, Taylor and Yates, (eds.) Discourse as Data: A guide for analysis, pp. 311-330 Milton Keynes: The Open University

Telegraph, (2007, February 22) The Town where ASBOs are for the ElderlyEastbourne Retrieved from www.telegraph.co.uk/news/uknews/1543428/The-town-where-asbos-are-for-the elderly.html

Telegraph, (2010a, April 19) Extreme sports killing the over 70s Retrieved from http://www.telegraph.co.uk/news/7603605/Extreme-sports-killing-the-over-70s.html

Telegraph, (2010b, August 17) Grim future for younger workers in pensioner ghettos Retrieved from

http://www.telegraph.co.uk/finance/personalfinance/7953602/Grim-future-for-youngworkers-in-pensioner-ghettos.html

Thomas, B. and Dorling, D., (2007) Identity in Britain: A Cradle-to-Grave Atlas Polity Press

Thorpe, M., (1999) Marginalization and resistance through the prism of retirement In Hearn, J. and Roseneil, S., (eds.) Consuming Cultures: Power and Resistance Basingstoke: MacMillan

Tiesdell, S., Oc, T. and Heath, T., (1996) Revitalising Historic Urban Quarters

Oxford: Butterworth-Heinemann

Torres-Gil, F.M., (1992) The New Aging: Politics and Change in America

New York: Auburn House

Tourism South East Research Unit, (2009) The Economic Impact of Tourism - Rother 2009

Hampshire: Tourism South East

Travers, A., Tunstall, R., Whitehad, C. and Pruvot, S., (2007) Population Mobility and Service Provision: A Report for London Council London: LSE London

Twenge, J.M. and Campbell, S.M., (2008) Generational differences in psychological traits and their impact on the workplace Journal of Management Psychology, Vol no. 23, 
pp. 862-877

Twenge, J.M., Campbell, S.M., Hoffman, B.J. and Lance, C.E., (2010) Generational Differences in Work Values, Leisure and Extrinsic Values Increasing, Social and Intrinsic Values Decreasing Journal of Management, Vol 36 no. 5, pp.1117-1142

URBED: Urban and Economic Development Group, (1994) Vital and Viable Town Centres: Meeting the Challenge

London: HMSO

Uren, Z. and Goldring, S., (2007) Migration Trends at Older Ages in England and Wales Population Trends, Vol no. 130, pp. 31-40

Urry, J., (1987) Holiday Making, Cultural Change and Seaside Lancaster Regionalism Group 1987 Working Paper no 22, 23

Urry J., (1994) Time, Leisure and Social Identity Time Society Vol no. 3 (2), pp. 131-149

Urry, J., (1995) Consuming Places

London: Routledge

Urry, J., (1997) Cultural change and the seaside resort In Shaw G. and Williams A.M., (eds.) The Rise and Fall of British Coastal Resorts, pp. 102-113

London: Pinter

Urry, J., (2000) Sociology beyond Societies

London: Routledge

Urry, J., (2002) The Tourist Gaze ( $2^{\text {nd }}$ ed.)

London: Sage Publications Ltd

Urry, J., (2003) Global Complexity

Cambridge: Polity Press

U.S. Bureau of the Census (2008) American Community Survey Housing Trends www.census.gov/acs/www

Van Dam, F., Heins, S. and Elbersen, B.C., (2002) Lay discourses of the rural and stated and revealed preferences for rural living. Some evidence of the existence of a rural idyll in the Netherlands Journal of Rural Studies, Vol no. 18 (4), pp. 461-476

Veblen, T., (1953) The theory of the leisure class American Library (Original work published in 1899)

New York: New York

Vickers, D., Rees, P. and Birkin, M.,(2003) A new classification of UK Local Authorities using 2001 Census key statistics University of Leeds - Working Paper 03/03

School of Geography: University of Leeds

VisitBritain, (2003) Implications of an ageing population in Britain Foresight November 2003: pp. 4-6

VisitBritain, (2005) What is the Future of Domestic Tourism to 2015

London: England Research

VisitBritain, (2006) The Short Break Market - An Analysis

London: England Research

Von Wright, G.H., (1971) Explanation and Understanding In Silverman D., (ed.)

Qualitative Research: Theory, Method and Practice

London: Sage

Wagner, P., (1994) A Sociology of Modernity: Liberty and Discipline 
London: Routledge

Waite, N., (2009) An interview with Mr. N. Waite, Principal Planning Officer for Bexhill Borough Council and Rother District Council 1969-2009 21 ${ }^{\text {st }}$ October 2009

Wales Tourist Board, (1992) Prospects for Coastal Resorts - Paper for Discussion Cardiff: Wales Tourist Board

Walker, A., (2002) The ESRC Growing Older Research Programme Social Policy and Society, Vol no. 1, pp. 129-138

Wall, G. and Mathieson, A., (2006) Tourism: Change, Impacts and Opportunities (2 ${ }^{\text {nd }}$ ed.)

London: Pearson Education Ltd

Wallis, J., (2006) Housing Options for Older People Pilot Project Report

Weymouth: Anchor Staying Put

Wallis, J., (2008) Housing Options for Older People Interim Project Report

Weymouth: Anchor Staying Put

Walsh, J., (1998) Across the Generation An Age Concern report into grandparent/grandchild relationships published to celebrate Grandparents' Day Retrieved from http://www.legacyproject.org/grandparentsdaykit/part3/gpday3.4.html

Walton, J.K., (1983) The English Seaside Resort: A Social History 1750-1914 Leicester: Leicester University Press

Walton, J.K., (2000) The British Seaside - Holidays and resorts in the twentieth century Manchester: Manchester University Press

Walton, J.K., (2009) Prospects in tourism history: Evolution, state of play and future developments Tourism Management December 2009, Vol. 30, Issue 6, pp. 783-793

Walton, J.K. and Browne, P., (2010) Coastal Regeneration in English Resorts Coastal Communities Alliance

BPR Publishers

Walvin, J., (1978) Beside the Seaside: A Social History of the Popular Seaside Holiday London: Allen Lane

Warnken, J., Russell R. and Faulkner, B., (2003) Condominium, developments in maturing destinations: potentials and problems of long-term sustainability Tourism Management April 2003, Vol no. 24 (2), pp. 155-168

Warnes, A.M., (1983a) Variations in the propensity among older persons to migrate: evidence and implications Journal of Applied Gerontology, Vol no. 2, pp. 20-27

Warnes A.M., (1983b) Migration in late working age and early retirement Socioeconomic Planning Sciences, pp. 291-302

Warnes, A.M., King, R., Williams, A.M. and Patterson, G., (1999) The well-being of British expatriate retirees in southern Europe Ageing and Society, Vol no. 19 (6), pp. 717-40

Warnes, A.M. and Law, C.M., (1982) The destination decision in retirement migration In Warnes, A.M., (ed.) Geographic perspectives on the elderly, pp. 53-81

Chichester: Wiley

Warnes, T. and Mclnerney, B., (2004) The English Regions and Population AgeingInformation for Policy and Governance: Requirements and Availability A Study commissioned by Age Concern England and the English Regions Network Regions for 
All Ages Programme researched by the Sheffield Institute for Studies on Ageing London: Age Concern Books

Watten, J.K., (1998) Blackpool

Edinburgh: Edinburgh University Press; Lancaster; Carnegie Publishing

Watten, J.K., (2000) The British Seaside: Holidays and Resorts in the Twentieth Century Manchester University Press

Weber, M., (1968) Economy and Society

Berkeley: University of California Press

Webster, L. and Mertova, P., (2007) Using Narrative Enquiry as a Research Method London and New York: Routledge

Wetherell, M., Taylor, S. and Yates, S. J., (2001) Discourse on Data: A Guide for Analysis

London: Sage Publications Ltd

Whiteleaf, P., (2010) Employers need to come to terms with an ageing workforce Professional Manager, Vol no. 19 (3)

Williams, A.M. and Hall, C.M., (2002) Tourism, Migration, Circulation and Mobility: the contingencies of time and place In Williams, A.M. and Hall, C.M., (eds.) Tourism and migration: new relationships between production and consumption, pp. 1-60 London: Kluwer Academic Publishers

Williams, A.M., King, R. and Warnes, A.M., (1997) A Place in the Sun; International Retirement Migration from Northern to Southern Europe

European Urban and Regional Studies, Vol no. 4 (2), pp. 115-134

Williams, A.M. and Shaw, G., (1997) Riding the big dipper: The rise and decline of the British seaside resort in the twentieth century In Shaw G. and Williams A.M., (eds.) The Rise and Fall of British Coastal Resorts, pp. 1-18

London: Pinter

Willig, C., (2001) Introducing Qualitative Research in Psychology, Adventures in theory and method

Buckingham: Open University Press

Willig, C., (2003) Discourse Analysis In Smith J. A., (ed.) Qualitative Psychology: A Practical Guide to Research Methods

London, Thousand Oakes, New Delhi: Sage

Wilson, L.B. and Simson, S.P., (2006) Civic Engagement and the Baby Boomer Generation (ed.) Research, Policy and Practice Perspectives London: Haworth Press

Wiseman, R.E., (1980) Why older people move Research on Aging, Vol no. 2 (2), pp. 141-54

Wolpert, J., (1965) Behavioural Aspects of the decision to migrate In Robinson, V., (1996) Geography and Migration

Cheltenham: Edward Elgar Publishing Ltd

Woodside, A., (2010) Case Study Research Theory, Methods and Practice London: Emerald Group Publishing Ltd

Yin, R.K., (1981a) The case study as a serious research strategy Knowledge: Creation, Diffusion, Utilization, Vol no. 3, pp. 97-114

Yin, R.K., (1981b) The case study crisis: Some answers Administrative Science Quarterly, Vol no. 26, pp. 58-65 
Yin, R.K., (1994) Case Study Research: Design and Methods

London: Sage Publication

Yin, R.K., (2003a) Applications of case study research ( $2^{\text {nd }}$ ed.)

Thousand Oaks, California: Sage

Yin, R.K., (2003b) Case Study Research. Design and Methods ( $3^{\text {rd }}$ ed.)

Thousand Oaks, California: Sage

Yin R.K., (2009) Case Study Research Design and Methods (4 ${ }^{\text {th }}$ ed.)

London: Sage Publications

Yin, R.K. and Davis, D., (2007) Adding new dimensions to case study evaluations. The case of evaluating comprehensive reforms In Julnes G. and Rog D.J., (eds.) Informing federal policies for evaluation methodology New Directions in Program Evaluation, Vol no. 113 , pp. $75-93$

San Francisco: Jossey-Bass

Zimon, J., (2000) Evolutionary models for technological change In J. Zimon (ed.)

Technological Innovation as an Evolutionary Process, pp. 3-12

Cambridge: Cambridge University Press

Zukin, S., (1982) Loft Living: Culture and Capital in Urban Change

Baltimore, MD: Johns Hopkins University Press

Zukin, S., (1995) The Cultures of Cities

Oxford and Cambridge. MA: Blackwell

Zukin, S., (1998) Urban lifestyles, diversity and standardisation in spaces of consumption Urban Studies, Vol no. 35, pp. 825-839 


\section{Appendix 1}

\section{Satisfaction ratings for English Local Authorities' - Categories for coastal towns' classification (2003)}

\begin{tabular}{|l|c|c|l|l|l|l|l|l|}
\hline & \multicolumn{2}{|c|}{ Question } & & \multicolumn{2}{|c|}{ Question } & \multicolumn{2}{|c|}{ Question } \\
\hline Coastal resorts & 1 & 2 & $\begin{array}{l}\text { Aged coastal } \\
\text { extremities }\end{array}$ & 1 & 2 & $\begin{array}{l}\text { Aged coastal } \\
\text { resorts }\end{array}$ & 1 & 2 \\
\hline Blackpool UA & 72 & 53 & Adur & 85 & 64 & Arun & 84 & 62 \\
\hline Bournemouth UA & 82 & 49 & Allerdale & 86 & 73 & Christchurch & 92 & 65 \\
\hline Eastbourne & 85 & 56 & Carlisle & 83 & 65 & East Devon & 89 & 66 \\
\hline Hastings & 75 & 57 & Dover & 81 & 62 & North Norfolk & 87 & 64 \\
\hline Southend-on-Sea & 83 & 55 & East Lindsey & 84 & 68 & Rother & 86 & 66 \\
\hline Thanet & 70 & 55 & $\begin{array}{l}\text { Great } \\
\text { Yarmouth }\end{array}$ & 80 & 59 & Tendring & 79 & 64 \\
\hline Torbay Unitary & 82 & 56 & Isle of Wight & 84 & 63 & West Dorset & 91 & 70 \\
\hline Worthing & 80 & 53 & North Devon & 87 & 68 & West Somerset & 89 & 72 \\
\hline & & & Scarborough & 83 & 69 & & & \\
\hline & & & Shepway & 79 & 59 & & & \\
\hline & & & Torridge & 85 & 69 & & & \\
\hline & & & Waveney & 81 & 62 & & & \\
\hline & & $\begin{array}{l}\text { Weymouth/ } \\
\text { Portland }\end{array}$ & 85 & 60 & & & \\
\hline & & & Wyre & 84 & 68 & & 83 & 58 \\
\hline South east Av. & 83 & 58 & & & & & 80 & 59 \\
\hline England & 80 & 59 & & 83 & 58 & & & \\
\hline
\end{tabular}

'Source: Vickers et al., (2003) and Ipsos/MORI, (2008/09)'

Notes:

Question 1: The overall satisfaction with your local area as a place to live

Question 2: Feeling of belonging to your neighbourhood

Data unavailable for: Kerrier, Penwith, Restormel, Carrick, North Cornwall, Caradon,

Pembrokeshire Unitary Authority

Classification: In 2003 Vickers et al., produced an area classification of Local Authorities using the Office of National Statistics 2001 census data that places local authorities into one of four family groupings (Appendix 8). Within this classification Table 3 shows England's seaside towns' satisfaction ratings with 'Place'.

What is noticeable is that the Aged Coastal Extremities and Aged Coastal Resorts in the majority of cases score well against the England Average. Whereas, the Coastal Resorts score significantly below the English average on the question of 'feeling of belonging to the neighbourhood' and aside from Bournemouth and Worthing these seaside towns happen to fall into the category of England's below average deprivation rankings 


\section{Appendix 2}

\section{English Local Authorities identified categories for coastal towns and their life expectancy rates against the English Average, 2003}

\begin{tabular}{|c|c|c|c|c|c|c|c|c|}
\hline \multicolumn{3}{|c|}{ Coastal resorts } & \multicolumn{3}{|c|}{ Aged coastal extremities } & \multicolumn{3}{|c|}{ Aged coastal resorts } \\
\hline & $\bar{M}$ & $\mathrm{~F}$ & & $\mathrm{M}$ & $\mathrm{F}$ & & $\mathrm{M}$ & $\mathrm{F}$ \\
\hline English Av. & 77.7 & 81.9 & & 77.7 & 81.9 & & 77.7 & 81.9 \\
\hline & \multicolumn{2}{|c|}{$+/-$ av.age } & & \multicolumn{2}{|c|}{$+/-$ av.age } & & \multicolumn{2}{|c|}{$+/-$ av.age } \\
\hline Blackpool UA & -0.6 & -0.9 & Adur & 0.9 & -0.1 & Arun & 1.2 & 0.8 \\
\hline $\begin{array}{l}\text { Bournemouth } \\
\text { UA }\end{array}$ & 0.6 & 0.5 & Allerdale & -0.1 & -1.1 & Christchurch & 2.7 & 3.1 \\
\hline Eastbourne & 1.1 & 1.1 & Caradon* & 1.1 & 1.2 & East Devon & 3.1 & 1.9 \\
\hline Hastings & -1.2 & -1.2 & Carlisle & -0.4 & -0.3 & $\begin{array}{l}\text { North } \\
\text { Norfolk }\end{array}$ & 1.2 & 2.6 \\
\hline Worthing & 0.9 & -0.1 & Carrick* $^{*}$ & 1.6 & 2.1 & Rother ** & 1.8 & 1.4 \\
\hline Thanet & -0.7 & -0.2 & Dover & 0.6 & 0.1 & Tendring & 0.8 & 0.4 \\
\hline $\begin{array}{l}\text { Torbay } \\
\text { Unitary }\end{array}$ & 0.6 & 0.7 & East Lindsey & -0.1 & -0.1 & West Dorset & 2.4 & 2.2 \\
\hline $\begin{array}{l}\text { Southend- } \\
\text { on-Sea }\end{array}$ & $\overline{N A}$ & NA & $\begin{array}{l}\text { Great } \\
\text { Yarmouth }\end{array}$ & -0.1 & -0.1 & $\begin{array}{l}\text { West } \\
\text { Somerset }\end{array}$ & 1.8 & 2.6 \\
\hline & & & Isle of Wight & 1.3 & 1.2 & & & \\
\hline & & & Kerrier $^{*}$ & 0.8 & 0.7 & & & \\
\hline & & & $\begin{array}{l}\text { North } \\
\text { Cornwall }^{*}\end{array}$ & 1.9 & 1.2 & & & \\
\hline & & & North Devon & 0.2 & 1.4 & & & \\
\hline & & & Penwith & 1.0 & 0.5 & & & \\
\hline & & & Restormel $^{*}$ & 0.7 & 0.9 & & & \\
\hline & & & Scarborough & -0.1 & 0.2 & & & \\
\hline & & & Shepway & 0.7 & 0.8 & & & \\
\hline & & & Torridge & 1.6 & 1.5 & & & \\
\hline & & & Waveney & 1.5 & 1.0 & & & \\
\hline & & & $\begin{array}{l}\text { Weymouth/ } \\
\text { Portland }\end{array}$ & 3.1 & 2.3 & & & \\
\hline & & & Wyre & 0.2 & 0.2 & & & \\
\hline
\end{tabular}

'Source: Vickers, Rees and Birkin, (2003) and ONS life expectancy (2010)'

Note: * nowadays part of Cornwall Unitary Authority

$\mathrm{M}$ (male), $\mathrm{F}$ (female)

** Bexhill-on-Sea falls within the local authority area of Rother District Council 


\section{Appendix 3}

\section{Population mid-term estimates and age distribution for the principal England's seaside towns, 2006}

\begin{tabular}{|c|c|c|c|c|}
\hline & & \multicolumn{3}{|c|}{ \%age of total population } \\
\hline & Population & $0-15$ & $16-59 / 64$ & $60 / 65+$ \\
\hline Sidmouth & 13,700 & 13 & 45 & 43 \\
\hline Bexhill & 42,900 & 13 & 53 & 34 \\
\hline Minehead & 12,100 & 15 & 52 & 33 \\
\hline Swanage & 10,100 & 15 & 53 & 33 \\
\hline Clacton & 58,000 & 17 & 51 & 32 \\
\hline Dawlish/Teignmouth & 30,300 & 16 & 54 & 30 \\
\hline Burnham-on-Sea & 19,100 & 16 & 54 & 30 \\
\hline Bognor Regis & 42,300 & 15 & 55 & 30 \\
\hline Bridlington & 39,200 & 17 & 54 & 29 \\
\hline St Ives & 11,200 & 15 & 57 & 28 \\
\hline Gr'tr Worthing & 191,300 & 17 & 56 & 27 \\
\hline Skegness & 20,400 & 17 & 57 & 27 \\
\hline Isle of Wight & 138,500 & 17 & 57 & 26 \\
\hline Eastbourne & 94,900 & 17 & 57 & 26 \\
\hline Torbay & 133,200 & 17 & 57 & 26 \\
\hline Exmouth & 34,200 & 17 & 57 & 26 \\
\hline Penzance & 21,600 & 17 & 58 & 26 \\
\hline Deal & 29,200 & 18 & 57 & 26 \\
\hline Southport & 90,400 & 17 & 57 & 25 \\
\hline Whitstable/Herne Bay & 69,700 & 19 & 56 & 25 \\
\hline Greater Blackpool & 264,600 & 18 & 58 & 25 \\
\hline Lowestoft & 63,900 & 19 & 57 & 24 \\
\hline Weymouth & 52,000 & 17 & 59 & 24 \\
\hline Greater Bournemouth & 335,500 & 17 & 59 & 24 \\
\hline Thanet & 122,300 & 19 & 56 & 24 \\
\hline Whitby & 13,700 & 17 & 59 & 24 \\
\hline Scarborough & 54,900 & 18 & 59 & 23 \\
\hline Falmouth & 21,100 & 17 & 60 & 23 \\
\hline Hastings & 86,400 & 18 & 59 & 23 \\
\hline Morecambe/Heysham & 50,800 & 19 & 58 & 23 \\
\hline Great Yarmouth & 58,300 & 19 & 58 & 23 \\
\hline Western-super-Mare & 76,300 & 18 & 58 & 23 \\
\hline Folkestone/Hythe & 60,100 & 19 & 59 & 23 \\
\hline Ilfracombe & 11,300 & 19 & 59 & 22 \\
\hline Newquay & 23,500 & 16 & 62 & 22 \\
\hline Southend-on-Sea & 159,900 & 19 & 59 & 21 \\
\hline Whitby Bay & 38,400 & 19 & 60 & 21 \\
\hline Greater Brighton & 284,300 & 16 & 66 & 18 \\
\hline English seaside towns & $2,877,200$ & 17 & 58 & 24 \\
\hline South East & $8,237,800$ & 19 & 62 & 19 \\
\hline England & $50,762,900$ & 19 & 62 & 19 \\
\hline
\end{tabular}

'Source: ONS taken from Beatty et al., (2008)' 


\section{Appendix 4}

\begin{tabular}{|c|c|c|}
\hline \multicolumn{3}{|c|}{ Vickers et al., Classification of Local Authority Areas, 2003} \\
\hline Families (4) & Groups (13) & Classes (26) \\
\hline \multirow{7}{*}{$\begin{array}{l}\text { A: Urban UK } \\
\text { (103 LA's - 35.8\% } \\
\text { population) }\end{array}$} & $\begin{array}{l}\text { A1: Industrial Legacy } \\
\text { (38 LA's }-9.4 \% \text { population }\end{array}$ & $\begin{array}{l}\text { A1a: Industrial Legacy } \\
\text { (38 LA's }-9.4 \% \text { population) }\end{array}$ \\
\hline & \multirow{4}{*}{$\begin{array}{l}\text { A2: Established Urban } \\
\text { Centres } \\
\text { (43 LA's }-17.7 \% \\
\text { population) }\end{array}$} & $\begin{array}{l}\text { A2a: Struggling Urban } \\
\text { Manufacturing } \\
\text { (14 Las }-5.6 \% \text { population) }\end{array}$ \\
\hline & & $\begin{array}{l}\text { A2b: Regional Centres } \\
\text { ( } 6 \text { Las }-3 \% \text { population) }\end{array}$ \\
\hline & & $\begin{array}{l}\text { A2c: Multicultural England } \\
\text { (13 LA's }-6.1 \% \text { population) }\end{array}$ \\
\hline & & $\begin{array}{l}\text { A2d: M8 Corridor } \\
\text { (10 LA's - 3\% population) }\end{array}$ \\
\hline & \multirow{2}{*}{$\begin{array}{l}\text { A3: Young and Vibrant } \\
\text { Cities } \\
\text { (22 LA's }-8.7 \% \text { population) }\end{array}$} & $\begin{array}{l}\text { A3a: Redeveloping Urban Centres } \\
\text { (14 LA's }-6.7 \% \text { population) }\end{array}$ \\
\hline & & $\begin{array}{l}\text { A3b: Young Multicultural } \\
\text { (5 LA's }-2 \% \text { population) }\end{array}$ \\
\hline \multirow{9}{*}{$\begin{array}{l}\text { B: Rural UK } \\
\text { (205 LA's - 36.2\% } \\
\text { population) }\end{array}$} & \multirow{3}{*}{$\begin{array}{l}\text { B1: Rural Britain } \\
\text { (93 LA's }-14.7 \% \\
\text { population) }\end{array}$} & $\begin{array}{l}\text { B1a: Rural Extremes } \\
\text { (24 LA's }-2.7 \% \text { population) }\end{array}$ \\
\hline & & $\begin{array}{l}\text { B1b: Agricultural Fringe } \\
\text { (35 LA's }-5.8 \% \text { population) }\end{array}$ \\
\hline & & $\begin{array}{l}\text { B1c: Rural Fringe } \\
\text { (39 LA's }-6.2 \% \text { population) }\end{array}$ \\
\hline & \multirow[t]{3}{*}{$\begin{array}{l}\text { B2: Coastal Britain } \\
\text { (44 LA's }-7.6 \% \text { population) }\end{array}$} & $\begin{array}{l}\text { B2a: Coastal Resorts } \\
\text { (8 LA's }-1.7 \% \text { population }\end{array}$ \\
\hline & & $\begin{array}{l}\text { B2b: Aged Coastal Extremities } \\
\text { (28 LA's }-1.7 \% \text { population) }\end{array}$ \\
\hline & & $\begin{array}{l}\text { B2c: Aged coastal resorts } \\
\text { (8 LA's }-3 \% \text { population) }\end{array}$ \\
\hline & \multirow[t]{2}{*}{$\begin{array}{l}\text { B3: Averageville } \\
\text { (67 LA's }-14 \% \text { population) }\end{array}$} & $\begin{array}{l}\text { B3a: Mixed Urban } \\
\text { (41 LA's }-8.8 \% \text { population) }\end{array}$ \\
\hline & & $\begin{array}{l}\text { B3b: Typical Towns } \\
\text { (26 LA's - 5.2\% population) }\end{array}$ \\
\hline & $\begin{array}{l}\text { B4: Isles of Scilly } \\
\text { (1 LA - .0037 population) }\end{array}$ & $\begin{array}{l}\text { B4a: Isles of Scilly } \\
\text { (1 LA }-.0037 \text { population) }\end{array}$ \\
\hline \multirow{3}{*}{$\begin{array}{l}\text { C: Prosperous } \\
\text { Britain } \\
\text { ( } 77 \text { LA's }-16.3 \% \\
\text { population) }\end{array}$} & \multirow[t]{2}{*}{$\begin{array}{l}\text { C1: Prosperous Urbanites } \\
\text { (23 LA's }-5.4 \% \text { population) }\end{array}$} & $\begin{array}{l}\text { C1a: Historic Cities } \\
\text { (3 LA's }-2.7 \% \text { population) }\end{array}$ \\
\hline & & $\begin{array}{l}\text { C1b: Thriving Outer London } \\
\text { (10 LA's }-2.7 \% \text { population) }\end{array}$ \\
\hline & $\begin{array}{l}\text { C2: Commuter Belt } \\
\text { (54 LA's }-10.9 \% \\
\text { population) }\end{array}$ & $\begin{array}{l}\text { C2a: The Commuter Belt } \\
\text { (54 LA's }-10.9 \% \text { population) }\end{array}$ \\
\hline \multirow[t]{5}{*}{$\begin{array}{l}\text { D: Urban London } \\
\text { (26 LA's }-9.6 \% \\
\text { population) }\end{array}$} & $\begin{array}{l}\text { D1: Multicultural Outer } \\
\text { London } \\
\text { (11 LA's }-4.4 \% \text { population) }\end{array}$ & $\begin{array}{l}\text { D1a: Multicultural Outer London } \\
\text { (11 LA's }-4.4 \% \text { population) }\end{array}$ \\
\hline & \multirow{2}{*}{$\begin{array}{l}\text { D2: Mercantile Inner } \\
\text { London } \\
\text { (7 LA's }-2 \% \text { population) }\end{array}$} & $\begin{array}{l}\text { D2a: Central London } \\
\text { (6 LA's }-1.9 \% \text { population) }\end{array}$ \\
\hline & & $\begin{array}{l}\text { D2b: City of London } \\
\text { (1 LA }-.01 \% \text { population) }\end{array}$ \\
\hline & \multirow[t]{2}{*}{$\begin{array}{l}\text { D3: Cosmopolitan Inner } \\
\text { London } \\
\text { (8 LA's }-3.2 \% \text { population) }\end{array}$} & $\begin{array}{l}\text { D3a: Afro-Caribbean Ethnic } \\
\text { Borough } \\
\text { ( } 5 \text { LA's }-2 \% \text { population) }\end{array}$ \\
\hline & & $\begin{array}{l}\text { D3b: Multicultural Inner London } \\
\text { (3 LA's }-1.2 \% \text { population) }\end{array}$ \\
\hline
\end{tabular}




\begin{tabular}{|c|c|c|}
\hline \multicolumn{3}{|c|}{ English Local Authorities categories for coastal towns, 2003} \\
\hline $\begin{array}{c}\text { Class B2a } \\
1.7 \% \text { population in cluster }\end{array}$ & $\begin{array}{c}\text { Class B2b } \\
4.6 \% \text { population in cluster }\end{array}$ & $\begin{array}{c}\text { Class B2c } \\
3 \% \text { population in cluster }\end{array}$ \\
\hline Coastal resorts & Aged coastal extremities & Aged coastal resorts \\
\hline Blackpool UA & Adur & Arun ${ }^{* 3^{3}}$ \\
\hline Bournemouth UA & Allerdale & 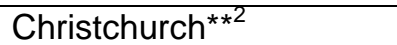 \\
\hline Eastbourne & Caradon* & East Devon ${ }^{\star * 1}$ \\
\hline Hastings & Carlisle & North Norfolk \\
\hline Southend-on-Sea & Carrick* & Rother \\
\hline Thanet & Dover & Tendring**4 $^{* 4}$ \\
\hline Torbay Unitary & East Lindsey & West Dorset ${ }^{\star * 5}$ \\
\hline Worthing & Great Yarmouth & West Somerset \\
\hline & Isle of Wight & \\
\hline & Kerrier* & \\
\hline & North Cornwall* & \\
\hline & \begin{tabular}{|l|} 
North Devon \\
\end{tabular} & \\
\hline & Pembrokeshire UA & \\
\hline & \begin{tabular}{|l|} 
Penwith \\
\end{tabular} & \\
\hline & Restormel $^{*}$ & \\
\hline & Scarborough & \\
\hline & Shepway & \\
\hline & \begin{tabular}{|l|} 
Torridge \\
\end{tabular} & \\
\hline & Waveney & \\
\hline & Weymouth/Portland & \\
\hline & Wyre & \\
\hline
\end{tabular}

'Source: Vickers et al., (2003)'

* nowadays part of Cornwall Unitary Authority

** denotes (in order) the local authorities most similar to Rother

The characteristics of these categories are as follows:

Coastal Resorts (B2a):

- Contain local authorities with large towns/cities that are holiday centres

- High number of very old people, health below national average which can be linked to large number of pensioners, many of whom live alone

- Bed-sits are more common than the average

- Significant number of homes with 2 adults and no children

Aged Coastal Extremities (B2b):

- Contain local authorities which are on the coast but don't contain any urban centres of any great size

- Characterised by aged population with a below average level of health

- A higher than expected number of homes without central heating

- Many pensioners live alone

Aged Coastal Resorts (B2c):

- Contain local authorities which have several small towns but no major urban centre. Many areas in this cluster contain coastal resorts which are in decline

- Very old population structure with high portions of pensioners living alone

- Many households with two adults and no children and a low number of dependent children

- Higher than expected number of people who are self employed 
Appendix 5

\begin{tabular}{|c|c|c|c|}
\hline \multicolumn{4}{|c|}{ Net migrants and net migration rates by district classification, 2000-01 } \\
\hline $\begin{array}{l}\text { District Classification } \\
\text { (Family, Group, Class) }\end{array}$ & Net migrants & $\begin{array}{c}\text { Total } \\
\text { population }\end{array}$ & $\begin{array}{l}\text { Net mig. Rate } \\
\text { (per } 1000 \\
\text { population) }\end{array}$ \\
\hline A: Urban UK & $-4,842$ & $20,607,931$ & -0.23 \\
\hline A1: Industrial Legacy & $-5,263$ & $5,512,854$ & -0.95 \\
\hline A1a: Industrial Legacy & $-5,263$ & $5,512,854$ & -0.95 \\
\hline A2: Established Urban Centres & $-16,829$ & $9,986,190$ & -1.69 \\
\hline A2a: Struggling Urban & $-8,292$ & $2,986,766$ & -2.78 \\
\hline \multicolumn{4}{|l|}{ Manufacturing } \\
\hline A2b: Regional Centres & 3,195 & $1,626,910$ & 1.96 \\
\hline A2c: Multicultural England & $-11,264$ & $3,600,034$ & -3.13 \\
\hline A2d: M8 Corridor & -468 & $1,722,480$ & -0.26 \\
\hline A3: Young and Vibrant Cities & 17,250 & $5,108,887$ & 3.38 \\
\hline A3a: Redeveloping Urban & 15,395 & $3,957,213$ & 3.89 \\
\hline \multicolumn{4}{|l|}{ Centres } \\
\hline A3b:Young Multicultural & 1,855 & $1,151,674$ & 1.61 \\
\hline \multirow{9}{*}{$\begin{array}{l}\text { B: Rural UK } \\
\text { B1: Rural Britain } \\
\text { B1a: Rural Extremes } \\
\text { B1b: Agricultural Fringe } \\
\text { B1c: Rural Fringe } \\
\text { B2: Coastal Britain } \\
\text { B2a: Coastal Resorts } \\
\text { B2b: Aged coastal Extremities } \\
\text { B2c: Aged Coastal Resorts }\end{array}$} & 57,947 & $21,281,909$ & 2.72 \\
\hline & 30,653 & $8,623,584$ & 3.55 \\
\hline & 1,067 & $1,587,196$ & 0.67 \\
\hline & 14,762 & $3,414,313$ & 4.32 \\
\hline & 14,842 & $3,622,075$ & 4.09 \\
\hline & 29,296 & $4,445,168$ & 6.59 \\
\hline & 7,231 & 994,660 & 7.27 \\
\hline & 13,987 & $2,689,628$ & 5.20 \\
\hline & 8,078 & 760,880 & 10.62 \\
\hline B3: Averageville & $-2,040$ & $8,211,021$ & -0.25 \\
\hline B3a: Mixed Urban & $-2,311$ & $5,179,434$ & -0.45 \\
\hline B3b: Typical Towns & 271 & $3,031,587$ & 0.09 \\
\hline B4: Isles of Scilly & 38 & 2,136 & 17.79 \\
\hline B4a: Isles of Scilly & 38 & 2,136 & 17.79 \\
\hline \multirow{6}{*}{$\begin{array}{l}\text { C: Prosperous Britain } \\
\text { C1: Prosperous Urbanites } \\
\text { C1a: Historic Cities } \\
\text { C1b: Thriving Outer London } \\
\text { C2: Commuter Belt } \\
\text { C2a: The Commuter Belt }\end{array}$} & $-4,983$ & $9,570,686$ & -0.52 \\
\hline & 1,844 & $3,169,458$ & 0.58 \\
\hline & 5,606 & $1,608,714$ & 3.48 \\
\hline & $-3,762$ & $1,560,744$ & -2.41 \\
\hline & $-6,827$ & $6,401,228$ & -1.07 \\
\hline & $-6,827$ & $6,401,228$ & -1.07 \\
\hline \multirow{3}{*}{$\begin{array}{l}\text { D: Urban London } \\
\text { D1: Multicultural Outer London } \\
\text { D1a: Multicultural Outer }\end{array}$} & $-48,122$ & $5,643,200$ & -8.53 \\
\hline & $-20,947$ & $2,613,603$ & -8.01 \\
\hline & $-20,947$ & $2,613,603$ & -8.01 \\
\hline \multicolumn{4}{|l|}{ London } \\
\hline D2: Mercantile Inner London & $-11,705$ & $1,146,832$ & -10.21 \\
\hline D2a: Central London & $-11,725$ & $1,139,651$ & -10.29 \\
\hline D2b: City of London & 20 & 7,181 & 2.79 \\
\hline D3: Cosmopolitan Inner London & $-15,470$ & $1,882,765$ & -8.22 \\
\hline D3a: Afro-Caribbean Ethnic & $-7,164$ & $1,179,278$ & -6.07 \\
\hline $\begin{array}{l}\text { Borough } \\
\qquad \text { D3b: Multicultural Inner London }\end{array}$ & $-8,306$ & 703,487 & -11.81 \\
\hline
\end{tabular}

'Source: Dennett and Stillwell, (2009)' 


\section{Appendix 6 \\ Net migrants and net migration rates by district classification - broad age groups, 2000-01}

The patterns of the youngest age group, $0-15$ year olds (columns 2-3), and 30-44 year olds (columns 6-7) are broadly similar, principally because the majority of $0-15$ year old migrants will be migrating with parents who are very likely to fall into the 30-44 age category. There is a clear pattern of net out-migration from urban areas, London especially while net in-migration can be observed in all areas defined as Rural UK. There is also net inmigration to areas defined as Commuter Belt, as individuals wishing to maintain city jobs move out to areas that are perceived as more appropriate for raising their families. Paradoxically, the highest migration rates of gain for the 0-15 year olds and 30-44 year olds are to be found in the Aged Coastal Resorts (column 3 - 16.21 and column 7 - 14.29 respectively).

In contrast to the $0-15$ age group, the 16-29 age group pattern of net migration is virtually the opposite (columns 4-5). In Classes within Rural and Coastal Britain Groups (with exception of Coastal Resorts where there tend to be universities) net out-migration rates are around 25 people per 1000 of population. It appears that rates of net out-migration increase with rurality, with Rural Extremes experiencing almost double the rate of net outmigration than the Rural Fringe. Net in-migration rates are high for urban areas, particularly vibrant urban districts with universities and London.

The pattern of migration changes for the 45 to state pension age group those that make up the middle-lifers (columns 8-9) - identify that whilst there is still a noticeable net out-migration of individuals in this Group from London, the rates of net out-migration are lower (12.1 persons per 1000 population for this age group, compared with 20.2 at 30-44 for Urban London). Similarly, the rate of net in-migration to Rural UK is lower at around 5.9 persons per 1000 population, although rates are noticeably higher for Coastal Britain. Within this Group, the Class of Aged Coastal Resorts exhibits the highest net in-migration rate of 22.01 persons per 1000 respectively.

The final age group includes those of state pension age and above (columns 10-11) and again includes part of the middle-lifers and shows essentially that the overall migration patterns of this group are very similar to the 45 to state pension age group. This is characterised by net outmigration from Urban London and other built-up areas in Urban UK and Prosperous UK, and net immigration to Rural UK, especially the Coastal Resort areas. Whilst there is an overall net out-migration from Commuter Belt areas, this is lower (1.4 people per 1000 population) than the preceding age group. Furthermore, for a number of Commuter Belt districts in the Home Counties, the rate of migration has switched from negative in the 44state pension age group to positive in the oldest group - perhaps reflecting 
the movement of dependent elderly individuals to the homes of younger relatives who provide care.

Of particular note is the Aged Coastal Resorts Class (B2c) that, whilst not having the highest absolute numbers, they exhibit the highest net migration rate for all age classifications other than 16-29 year olds.

'Source: Dennett and Stillwell, (2009)' 


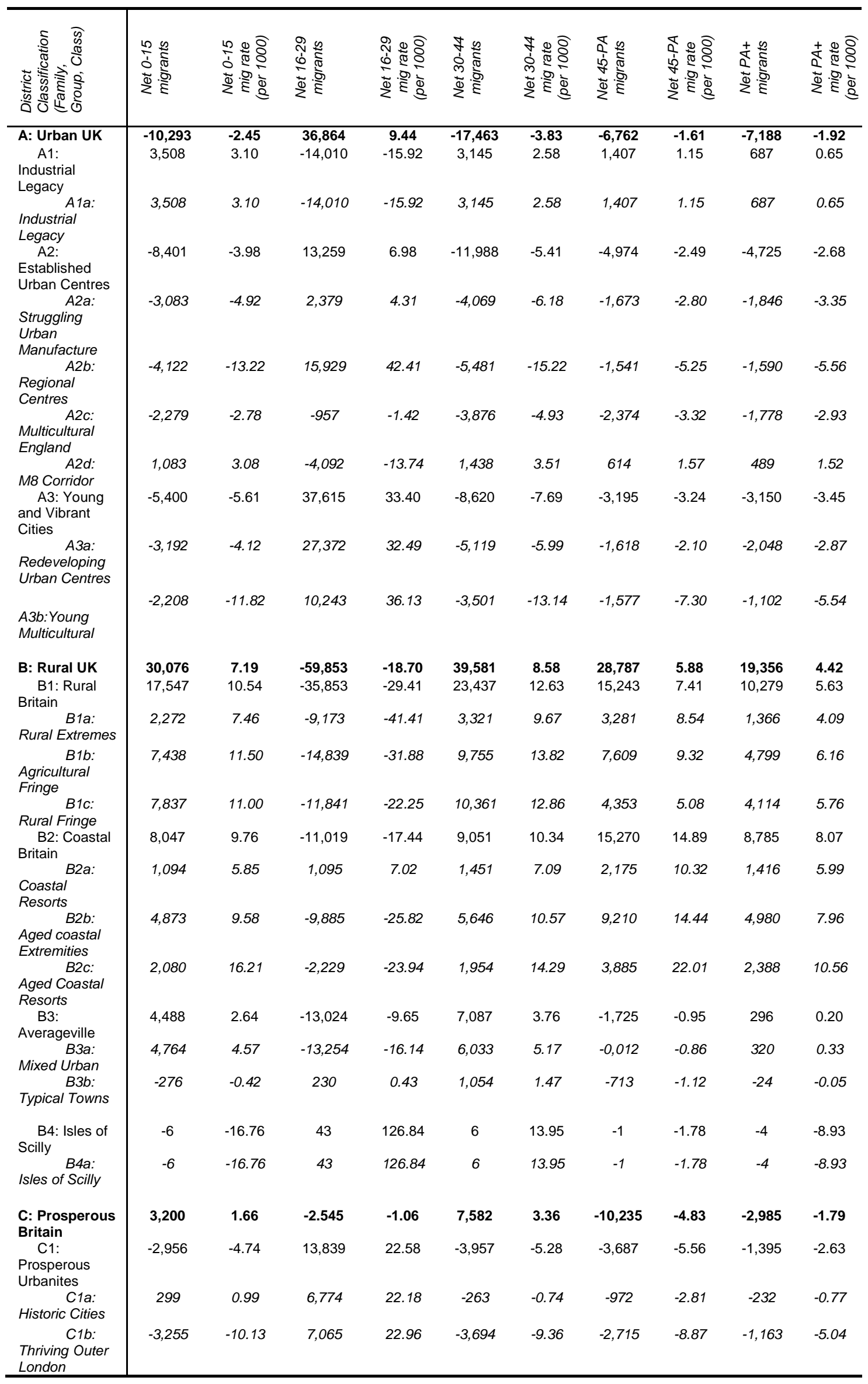




\begin{tabular}{|c|c|c|c|c|c|c|c|c|c|c|}
\hline 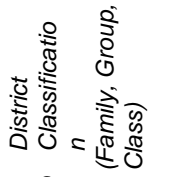 & 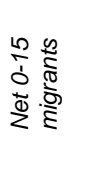 & 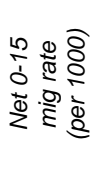 & 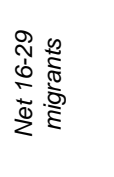 & 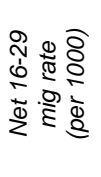 & 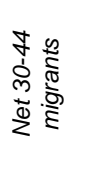 & 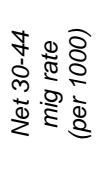 & 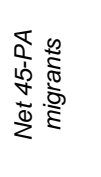 & 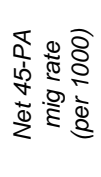 & 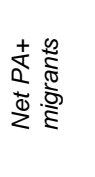 & 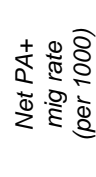 \\
\hline $\begin{array}{l}\text { C2: } \\
\text { Commuter Belt }\end{array}$ & 6,156 & 4.71 & $-16,384$ & -16.71 & 11,539 & 7.64 & $-6,548$ & -4.46 & $-1,590$ & -1.40 \\
\hline $\begin{array}{l}\text { C2a: } \\
\text { The Commuter } \\
\text { Belt }\end{array}$ & 6,156 & 4.71 & $-16,384$ & -16.71 & 11,539 & 7.64 & $-6,548$ & -4.46 & $-1,590$ & -1.40 \\
\hline $\begin{array}{l}\text { D: Urban } \\
\text { London }\end{array}$ & $-22,983$ & -20.08 & 25,534 & 19.71 & $-29,700$ & -20.24 & $-11,790$ & -12.11 & $-9,183$ & -12.05 \\
\hline $\begin{array}{c}\text { D1: } \\
\text { Multicultural }\end{array}$ & $-6,027$ & -10.89 & 293 & 0.55 & $-7,276$ & -11.32 & $-4,685$ & -9.50 & $-3,252$ & -8.29 \\
\hline $\begin{array}{c}\text { Outer London } \\
\text { D1a: } \\
\text { Multicultural }\end{array}$ & $-6,027$ & -10.89 & 293 & 0.55 & $-7,276$ & -11.32 & $-4,685$ & -9.50 & $-3,252$ & -8.29 \\
\hline $\begin{array}{l}\text { Outer London } \\
\text { D2: } \\
\text { Mercantile } \\
\text { Inner London }\end{array}$ & $-6,932$ & -37.52 & 11,799 & 38.93 & $-11,402$ & -35.77 & $-2,819$ & -14.69 & $-2,351$ & -15.84 \\
\hline $\begin{array}{c}\text { D2a: } \\
\text { Central London }\end{array}$ & $-6,890$ & -37.43 & 11,765 & 39.03 & $-11,396$ & -35.98 & $-2,855$ & -15.02 & $-2,349$ & -15.95 \\
\hline $\begin{array}{c}D 2 b: \\
\text { City of London }\end{array}$ & -42 & -62.69 & 34 & 20.62 & -6 & -3.05 & 36 & 20.04 & -2 & -1.82 \\
\hline $\begin{array}{l}\text { D3: } \\
\text { Cosmopolitan } \\
\text { Inner London }\end{array}$ & $-10,024$ & -24.67 & 13,442 & 29.18 & $-11,022$ & -21.79 & $-4,286$ & -14.85 & $-3,580$ & -16.17 \\
\hline $\begin{array}{l}\text { D3a: } \\
\text { Afro-Caribbean } \\
\text { Ethnic Borough }\end{array}$ & $-6,533$ & -26.63 & 11,202 & 39.74 & $-6,982$ & -20.98 & $-2,761$ & -15.23 & $-2,090$ & -15.45 \\
\hline $\begin{array}{l}\quad \text { D3b: } \\
\text { Multicultural } \\
\text { Inner London }\end{array}$ & $-3,491$ & -21.70 & 2,240 & 12.53 & $-4,040$ & -23.34 & $-1,525$ & -14.20 & $-1,490$ & -17.87 \\
\hline
\end{tabular}

Net migrants and net migration rates by district classification - broad age groups, 2000-01.

'Source: Dennett and Stillwell, (2009)' 


\begin{tabular}{|c|c|c|c|c|c|c|c|c|c|c|c|c|c|c|c|c|c|c|c|c|c|c|c|c|}
\hline & בे & & $\tilde{\Xi}$ & 음 & $\stackrel{n}{m}$ & m. & 冓 & $\begin{array}{l}\text { t } \\
\dot{m}\end{array}$ & ڤ్ర & 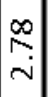 & 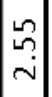 & 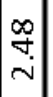 & 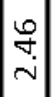 & & 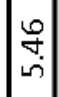 & $\left|\begin{array}{c}\mathscr{D} \\
\dot{\tau}\end{array}\right|$ & $\mid$ & $\begin{array}{l}\overrightarrow{-} \\
\stackrel{-}{+} \\
\dot{\sigma}\end{array}$ & $\begin{array}{l}\vec{J} \\
\stackrel{+}{+}\end{array}$ & 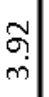 & ने. & $\left|\begin{array}{c}\Re \\
\infty \\
\dot{m}\end{array}\right|$ & & $\mid \begin{array}{l}\mid \overrightarrow{0} \\
\dot{m}\end{array}$ \\
\hline & $\pi$ & & : & $\tilde{D}$ & U & $\widetilde{\infty}$ & 5 & $\vec{\infty}$ & $\tilde{\infty}$ & $\bar{\infty}$ & $\bar{\infty}$ & $\hat{\infty}$ & $\widetilde{O}$ & & $\mid \overrightarrow{0}$ & $\vec{\infty}$ & $\vec{\infty}$ & U & $\widetilde{U}$ & -1 & 5 & $-\vec{\infty}$ & $\mathbb{S}$ & $\widetilde{U}$ \\
\hline & 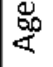 & & 5 & $\ddot{\infty}$ & 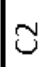 & U & $\tilde{m}$ & 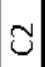 & $\tilde{\infty}$ & $\tilde{\infty}$ & $\tilde{\infty}$ & 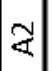 & $\bar{\infty}$ & & $\tilde{0}$ & $\sim$ & $\tilde{\infty}$ & 5 & $\vec{U}$ & 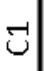 & $\widetilde{0}$ & $q$ & $\approx$ & $\tilde{\partial}$ \\
\hline & & & 苋 & 을 & 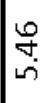 & 요 & $\begin{array}{l}\vec{\infty} \\
\dot{\gamma} \\
\end{array}$ & $\underset{+}{\stackrel{8}{*}}$ & $\begin{array}{l}0 \\
\stackrel{0}{+}\end{array}$ & $\begin{array}{l}\hat{b} \\
\dot{\sigma}\end{array}$ & 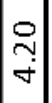 & $\begin{array}{l}\curvearrowright \\
\tilde{m}\end{array}$ & $\begin{array}{c}m \\
\tilde{m} \\
\dot{m}\end{array} \mid$ & & नุ & 命 & भُ & $\begin{array}{c}\vec{m} \\
\hat{\omega}\end{array}$ & $\begin{array}{l}2 \\
0 \\
0\end{array}$ & ન્| & 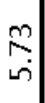 & $\mid \begin{array}{l}-\overrightarrow{0} \\
. \\
.\end{array}$ & 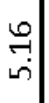 & $\begin{array}{c}0 \\
0 \\
0\end{array}$ \\
\hline 9 & $\underset{0}{7}$ & & $\approx$ & $\tilde{\infty}$ & ס & $ت$ & $\tilde{\sigma}$ & 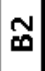 & $\vec{\infty}$ & $\overrightarrow{0}$ & $\tilde{a}$ & $\tilde{a}$ & ₹ & & $\overrightarrow{0}$ & $\circlearrowleft$ & $\vec{\infty}$ & m & -1 & $\vec{\infty}$ & $\checkmark$ & -1 & $\vec{\infty}$ & $\widetilde{\circlearrowleft}$ \\
\hline 18 & & & 5 & $\vec{\infty}$ & $\tilde{U}$ & $\widetilde{O}$ & 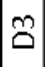 & $\ddot{\infty}$ & $\checkmark$ & $\tilde{\theta}$ & $\approx$ & 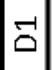 & $\ddot{\infty}$ & & $\tilde{s}$ & $ت$ & 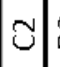 & $\Xi$ & ปี & ธิ & 5 & $ت$ & ₹ & ช \\
\hline 2 & $\mathbb{A}$ & & $\stackrel{8}{8}$ & $\stackrel{-1}{n}$ & $\stackrel{\infty}{\stackrel{\infty}{\circ}}$ & 亗 & $\begin{array}{l}\text { 굼 } \\
\text { m. }\end{array}$ & 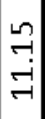 & 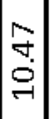 & $\begin{array}{c}2 \\
\hat{m}\end{array}$ & बें & ने & $\mid \begin{array}{l}\infty \\
0 \\
\infty \\
\infty\end{array}$ & & 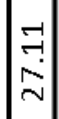 & $\begin{array}{l}\stackrel{0}{2} \\
\stackrel{\sim}{\sim}\end{array}$ & \begin{tabular}{l}
-1 \\
\multicolumn{1}{c}{} \\
-
\end{tabular} & 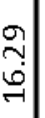 & 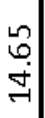 & $\begin{array}{l}\stackrel{?}{2} \\
\underset{\sim}{7}\end{array}$ & $\begin{array}{l}\stackrel{0}{\infty} \\
\stackrel{\infty}{-}\end{array}$ & $\begin{array}{l}0 \\
\stackrel{9}{9} \\
\stackrel{9}{-}\end{array}$ & $\begin{array}{l}\text { ने } \\
\text { ڤे }\end{array}$ & \begin{tabular}{l}
\multirow{2}{*}{} \\
\multirow{-}{*}{}
\end{tabular} \\
\hline के & $m$ & & $\mathscr{\Xi}$ & $\tilde{\sigma}$ & $\tilde{c}$ & 5 & 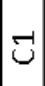 & 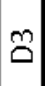 & $\approx$ & $\mathcal{O}$ & $\tilde{\infty}$ & ชิ & $\overrightarrow{0}$ & & $\tilde{a}$ & 5 & $\mathcal{N}$ & 5 & $\widetilde{U}$ & $ت$ & $\widetilde{O}$ & $-\vec{\infty}$ & $\tilde{\Xi}$ & $\vec{\infty}$ \\
\hline 1. & & & 5 & $\tilde{\theta}$ & $\tilde{a}$ & $\tilde{a}$ & $\widetilde{U}$ & 5 & $\vec{\infty}$ & $\Xi$ & \ุ & 5 & $\widetilde{\alpha}$ & & $\approx$ & $\tilde{\theta}$ & 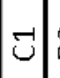 & ฮี & -1 & ธี & ธี & $\tilde{\infty}$ & $\widehat{\rho}$ & \& \\
\hline 8 & ปิ & & $\frac{8}{8}$ & $\begin{array}{l}\tilde{O} \\
\dot{\varphi} \\
\dot{q}\end{array}$ & 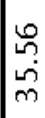 & ज्ञ & 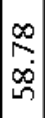 & 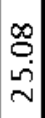 & 它 & $\begin{array}{l}\infty \\
\stackrel{\infty}{+} \\
\stackrel{\sim}{N}\end{array}$ & $\begin{array}{l}N \\
\stackrel{N}{\sim} \\
\stackrel{\sim}{2}\end{array}$ & 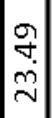 & $\frac{\vec{a}}{\mathrm{r}}$ & & $\begin{array}{l}0 \\
0 \\
\dot{j} \\
\end{array}$ & $\begin{array}{l}\wp \\
\sigma \\
\infty \\
\sim \\
\sim\end{array}$ & مै & $\begin{array}{l}\stackrel{m}{N} \\
\stackrel{\sim}{\sim}\end{array}$ & $\begin{array}{l}\infty \\
\stackrel{\infty}{0} \\
\stackrel{0}{0}\end{array}$ & 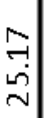 & 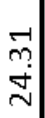 & مُ & $\begin{array}{c}\stackrel{\Delta}{N} \\
\stackrel{i}{N}\end{array}$ & 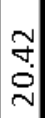 \\
\hline$\stackrel{0}{2}$ & 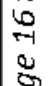 & & 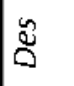 & $\Xi$ & $\ddot{a}$ & J & $z$ & 5 & $\tilde{a}$ & $\approx$ & $\approx$ & ธี & $q$ & & $\ddot{a}$ & 5 & $\mathcal{O}$ & จ & $\stackrel{m}{<}$ & -1 & $q$ & 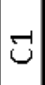 & ฉด & दै \\
\hline o & & & 5 & $\tilde{a}$ & $\approx$ & U & $\vec{\infty}$ & 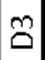 & 5 & $q$ & $\overrightarrow{0}$ & $\widetilde{\circlearrowleft}$ & $\widetilde{S}$ & & $\approx$ & 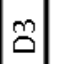 & $\vec{\Delta}$ & $\underline{\Xi}$ & -1 & $\widetilde{\Xi}$ & $\mathbb{U}$ & 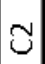 & 5 & $m$ \\
\hline 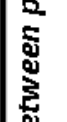 & 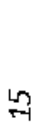 & & $\begin{array}{l}8 \\
0 \\
0\end{array}$ & f. & 守 & $\underset{\infty}{\underset{\infty}{+}}$ & 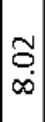 & $\begin{array}{l}m \\
6 \\
6\end{array}$ & 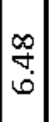 & 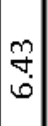 & $\mid \begin{array}{l}\mid \\
0 \\
0 \\
0\end{array}$ & 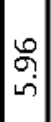 & में & & $\left|\begin{array}{l}\stackrel{0}{2} \\
\stackrel{2}{+} \\
-1\end{array}\right|$ & 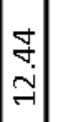 & : & $\begin{array}{l}\text { fे } \\
\text { ने } \\
\vec{g}\end{array}$ & 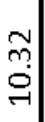 & సે. & $\begin{array}{l}\stackrel{n}{n} \\
\infty \\
\infty\end{array}$ & $\mid$ & 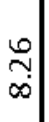 & รั. \\
\hline 15 & 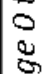 & & 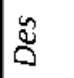 & 5 & $\tilde{\infty}$ & ป & 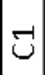 & $\tilde{\infty}$ & $\vec{\infty}$ & $\tilde{\sim}$ & $\vec{\infty}$ & ڤُ & $\mathcal{U}$ & & 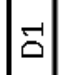 & $\sim$ & $\tilde{\theta}$ & 5 & $\checkmark$ & $\widetilde{\Psi}$ & $\vec{\infty}$ & $ت$ & $\mid-1$ & $\vec{\infty}$ \\
\hline 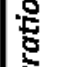 & & & $a$ & $\tilde{\theta}$ & $\vec{\infty}$ & $\tilde{a}$ & $\mathcal{O}$ & \& & $\checkmark$ & $\tilde{\infty}$ & 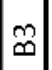 & $\overrightarrow{0}$ & U & & $\tilde{\theta}$ & $ت$ & $\approx$ & $\widetilde{\sigma}$ & $\approx$ & 5 & $\mathcal{\infty}$ & $\approx$ & $\checkmark$ & $q$ \\
\hline 咅 & & 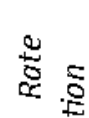 & Eั & 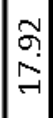 & 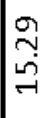 & $\begin{array}{l}\text { ने } \\
\text { ন }\end{array}$ & $\begin{array}{l}\infty \\
\stackrel{\infty}{\sim} \\
\underset{\sim}{\sim}\end{array}$ & 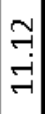 & $\begin{array}{l}\text { 영 } \\
\stackrel{9}{\circ} \\
\stackrel{1}{1}\end{array}$ & $\begin{array}{l}0 \\
0 \\
0 \\
\ddots\end{array}$ & $\begin{array}{l}\tilde{n} \\
\tilde{n} \\
\sigma\end{array}$ & 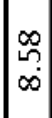 & $\infty$ & $\frac{0}{5}$ & $\begin{array}{l}\text { 어 } \\
\stackrel{-}{\leftrightarrow} \\
\stackrel{\sim}{\sim}\end{array}$ & 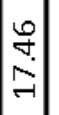 & 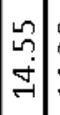 & 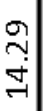 & $\begin{array}{l}\underset{7}{ت} \\
\underset{-}{ت}\end{array}$ & 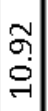 & $\begin{array}{l}\approx \\
\infty \\
\stackrel{\leftrightarrow}{-}\end{array}$ & 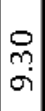 & $\stackrel{n}{\infty}$ & $\left|\begin{array}{c}\infty \\
+ \\
\infty\end{array}\right|$ \\
\hline 17 & 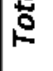 & .5 & 5 & $\approx$ & $\tilde{0}$ & J & 5 & $\tilde{\theta}$ & $\approx$ & $q$ & $\approx$ & $\approx$ & $\tilde{\sigma}$ & ${ }^{\circ}=-1$ & $\tilde{\theta}$ & $\overrightarrow{0}$ & $\overrightarrow{0}$ & $\mathcal{\triangle}$ & $ت$ & $\approx$ & $\mathcal{S}$ & $\checkmark$ & $\vec{\infty}$ & $\vec{\infty}$ \\
\hline 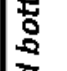 & & & & $\tilde{\theta}$ & $\approx$ & U & 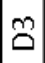 & 5 & 5 & $\ddot{\infty}$ & $\vec{\infty} \mid$ & $\mathcal{U}$ & J & 0 & $\approx$ & $\tilde{\theta}$ & $\approx$ & ت & 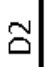 & $\tilde{a}$ & 5 & $\approx$ & $\tilde{\infty}$ & 2 \\
\hline & & & & & $\sim$ & m & o & תמ & . & & $\infty$ & $a$ & $\vec{F}$ & & -1 & $\sim$ & $m$ & $\nabla$ & n & ○) & 1 & $\infty$ & al & -1 \\
\hline
\end{tabular}

'Source: Dennett and Stillwell, (2009)' 


\section{Appendix 8}

\section{Employment by sector in England's principal seaside towns, 2006,}

(ranked by public administration)

\begin{tabular}{|c|c|c|c|c|c|}
\hline & \multicolumn{5}{|c|}{$\%$ of all employee jobs } \\
\hline & $\begin{array}{l}\text { Public } \\
\text { admin }\end{array}$ & $\begin{array}{l}\text { Distribution, } \\
\text { hotels and } \\
\text { restaurants }\end{array}$ & $\begin{array}{l}\text { Banking } \\
\text { and } \\
\text { Finance }\end{array}$ & $\begin{array}{c}\text { Manu- } \\
\text { facturing }\end{array}$ & $\begin{array}{c}\text { Other } \\
\text { services }\end{array}$ \\
\hline Whitley Bay & 48 & 31 & 9 & 2 & 10 \\
\hline Hastings and Bexhill & 43 & 23 & 14 & 10 & 10 \\
\hline BognorRegis & 40 & 31 & 15 & 3 & 11 \\
\hline Dawlish/Teignmouth & 40 & 32 & 9 & 2 & 17 \\
\hline Scarborough & 39 & 34 & 10 & 4 & 13 \\
\hline Thanet & 38 & 29 & 10 & 10 & 13 \\
\hline Clacton & 37 & 32 & 10 & 8 & 13 \\
\hline Eastbourne & 37 & 31 & 15 & 7 & 10 \\
\hline Bridlington & 36 & 36 & 7 & 6 & 15 \\
\hline Exmouth & 36 & 36 & 12 & 4 & 12 \\
\hline Penzance & 36 & 35 & 11 & 3 & 15 \\
\hline Deal & 35 & 36 & 11 & 2 & 16 \\
\hline Southport & 35 & 33 & 13 & 5 & 14 \\
\hline Gr'tr Blackpool & 35 & 30 & 14 & 7 & 14 \\
\hline Western-super-Mare & 34 & 32 & 16 & 5 & 13 \\
\hline Great Yarmouth & 34 & 31 & 11 & 7 & 17 \\
\hline Southend-on-Sea & 34 & 25 & 22 & 7 & 12 \\
\hline Gr'tr Worthing & 34 & 24 & 21 & 10 & 11 \\
\hline Weymouth & 33 & 39 & 13 & 2 & 13 \\
\hline Torbay & 33 & 35 & 13 & 6 & 13 \\
\hline Swanage & 33 & 35 & 12 & 4 & 15 \\
\hline Isle of Wight & 33 & 32 & 10 & 9 & 16 \\
\hline Sidmouth & 32 & 39 & 8 & 1 & 20 \\
\hline Whitstable/Herne Bay & 32 & 30 & 12 & 12 & 14 \\
\hline Whitby & 31 & 41 & 8 & 8 & 12 \\
\hline Lowestoft & 31 & 28 & 14 & 9 & 18 \\
\hline Folkestone/Hythe & 31 & 26 & 19 & 8 & 16 \\
\hline Ilfracombe & 30 & 31 & 6 & 16 & 17 \\
\hline Gr'tr Brighton & 30 & 24 & 26 & 4 & 16 \\
\hline Minehead & 29 & 49 & 7 & 4 & 11 \\
\hline Gr'tr Bournemouth & 28 & 28 & 22 & 10 & 12 \\
\hline Morecambe/Heysham & 27 & 34 & 11 & 9 & 19 \\
\hline Burnham-on-Sea & 27 & 29 & 7 & 25 & 12 \\
\hline Skegness & 24 & 42 & 8 & 9 & 17 \\
\hline Newquay & 23 & 55 & 9 & 2 & 11 \\
\hline Falmouth & 23 & 36 & 15 & 15 & 11 \\
\hline St Ives & 13 & 46 & 27 & 3 & 11 \\
\hline Seaside towns & 33 & 30 & 17 & 7 & 13 \\
\hline South east & 25 & 25 & 24 & 9 & 17 \\
\hline England & 26 & 24 & 22 & 11 & 17 \\
\hline
\end{tabular}

'Source: Annual Business Enquiry adapted from Beatty et al., (2008)'

Note: 'other services' includes such sectors as agriculture, construction, transport and communications, etc. 


\section{Appendix 9}

\begin{tabular}{|c|c|c|c|c|c|}
\hline & EMERGENCE & $\begin{array}{l}R \\
E \\
C \\
O \\
G \\
N \\
I \\
T \\
I \\
O \\
N\end{array}$ & RESTRUCTURING & $\begin{array}{c}\mathrm{S} \\
\mathrm{A} \\
\mathrm{T} \\
\mathrm{U} \\
\mathrm{R} \\
\mathrm{A} \\
\mathrm{T} \\
\mathrm{I} \\
\mathrm{O} \\
\mathrm{N}\end{array}$ & NEW CONCERNS \\
\hline Population & $\begin{array}{c}\text { Increasing } \\
\text { In-migration } \\
\text { (seasonal, amenity, } \\
\text { return, assistance) }\end{array}$ & & $\begin{array}{c}\text { Continued } \\
\text { In-migration } \\
\text { (changing age } \\
\text { structure) }\end{array}$ & & $\begin{array}{c}\text { Controlled growth } \\
\text { and overcrowding } \\
\text { (coping with ageing of } \\
\text { migrants) }\end{array}$ \\
\hline \multicolumn{6}{|l|}{ Correlates } \\
\hline Political & Involvement/activism & & $\begin{array}{c}\text { Political } \\
\text { reorganisation }\end{array}$ & & Co-operation/conflict \\
\hline Economic & Infusion of capital & & $\begin{array}{c}\text { Investment in local } \\
\text { development }\end{array}$ & & $\begin{array}{c}\text { Economic } \\
\text { balance/polarised } \\
\text { economy }\end{array}$ \\
\hline Social & $\begin{array}{l}\text { Challenge to social } \\
\text { order }\end{array}$ & & Social change & & $\begin{array}{l}\text { Social integration/ } \\
\text { Fragmentation }\end{array}$ \\
\hline Infrastructural & Increasing pressure & & $\begin{array}{l}\text { Infrastructure } \\
\text { development }\end{array}$ & & $\begin{array}{l}\text { Adequacy/overload } \\
\text { congestion }\end{array}$ \\
\hline Environmental & Increasing use & & $\begin{array}{l}\text { Regulation/ } \\
\text { conservation }\end{array}$ & & $\begin{array}{l}\text { Environmental } \\
\text { improvement/ } \\
\text { degradation }\end{array}$ \\
\hline AE & & & & & \\
\hline
\end{tabular}

'Source: Rowles and Watkins, (2001)' 
Appendix 10

Net in-migration of working age into seaside towns by age band, 19711991

\begin{tabular}{|l|r|r|}
\hline & Men & Women \\
\hline $16-24$ & 8,000 & 22,000 \\
\hline $24-34$ & 0 & 2,000 \\
\hline $35-44$ & 36,000 & 38,000 \\
\hline $45-54$ & 30,000 & 29,000 \\
\hline $55-64$ (men) & 37,000 & 18,000 \\
\hline $55-59$ (women) & & 109,000 \\
\hline
\end{tabular}

'Source: ONS taken from Beatty and Fothergill, (2004)' 


\section{Appendix 11}

\section{Employment and Income Status of Middle-life Participants}

\section{Employment and Income Status of Research Participants}

\begin{tabular}{|c|c|c|c|}
\hline Ref & Age & $\begin{array}{l}\text { Work/retired } \\
\text { (nature of career) }\end{array}$ & $\begin{array}{l}\text { Household } \\
\text { Income/an }\end{array}$ \\
\hline \multicolumn{4}{|l|}{$\mathbf{a}$} \\
\hline $103 a$ & 55 (f) & $\begin{array}{l}\text { Unemployed actively seeking work (public } \\
\text { administration) } \\
\text { husband retired }\end{array}$ & $<£ 15 k$ \\
\hline $104 a$ & $51(\mathrm{~m})$ & $\begin{array}{l}\text { Gatwick airport (supervisor) } \\
\text { wife lives in Spain }\end{array}$ & $£ 25-50 \mathrm{k}$ \\
\hline $106 a$ & $52(\mathrm{~m})$ & $\begin{array}{l}\text { Retired - ill health (bank manager) } \\
\text { single }\end{array}$ & $£ 15-25 k$ \\
\hline $116 a$ & $53(\mathrm{~m})$ & $\begin{array}{l}\text { Self-employed (p/t university lecturer, playwright, } \\
\text { consultant) } \\
\text { wife university lecturer }\end{array}$ & $£ 25-50 k$ \\
\hline $121 a$ & $55(\mathrm{~m})$ & $\begin{array}{l}\text { Semi-retired first aid consultant (Met police) } \\
\text { wife housewife }\end{array}$ & $£ 25-50 \mathrm{k}$ \\
\hline \multicolumn{4}{|l|}{ b } \\
\hline $102 b$ & 59 (f) & $\begin{array}{l}\text { Working (finance officer) } \\
\text { husband retired }\end{array}$ & £25-50k \\
\hline $113 b$ & 60 (f) & $\begin{array}{l}\text { Semi-retired ill health (NHS nurse) health consultant } \\
\text { husband ill health retirement }\end{array}$ & $£ 15-25 k$ \\
\hline $114 b$ & 58 (f) & $\begin{array}{l}\text { Retired (local govt librarian) } \\
\text { husband retired }\end{array}$ & $£ 25-50 k$ \\
\hline $115 b$ & $59(\mathrm{~m})$ & $\begin{array}{l}\text { Unemployed (surveyor) } \\
\text { single }\end{array}$ & $<£ 15 k$ \\
\hline $119 b$ & $\begin{array}{l}56(f) \\
(\text { Dis) }\end{array}$ & $\begin{array}{l}\text { Retired (disabled) (NHS nurse) } \\
\text { partner retired }\end{array}$ & £25-50k \\
\hline \multicolumn{4}{|l|}{ c } \\
\hline $101 \mathrm{c}$ & 65 (f) & $\begin{array}{l}\text { Retired (bookkeeper) } \\
\text { partner disabled }\end{array}$ & $£ 25-50 k$ \\
\hline $109 \mathrm{c}$ & $63(\mathrm{~m})$ & $\begin{array}{l}\text { Retired (Banking) } \\
\text { single divorced }\end{array}$ & £25-50k \\
\hline $110 c$ & $63(\mathrm{~m})$ & $\begin{array}{l}\text { Retired (Met police) } \\
\text { wife housewife }\end{array}$ & £25-50k \\
\hline $112 \mathrm{c}$ & $61(\mathrm{~m})$ & $\begin{array}{l}\text { Semi-retired (manufacturing) } \\
\text { wife retired }\end{array}$ & $>£ 50 k$ \\
\hline $118 \mathrm{c}$ & 65 (f) & $\begin{array}{l}\text { Retired (PA London) } \\
\text { husband retired }\end{array}$ & £25-50k \\
\hline $120 c$ & $\begin{array}{l}62(f) \\
(\text { Dis) }\end{array}$ & $\begin{array}{l}\text { Retired ill-health (NHS administration) } \\
\text { single divorced }\end{array}$ & $<£ 15 k$ \\
\hline \multicolumn{4}{|l|}{ d } \\
\hline $105 d$ & $70(\mathrm{~m})$ & $\begin{array}{l}\text { Retired (owner of company) } \\
\text { widowed }\end{array}$ & £25-50k \\
\hline $107 d$ & 69 (f) & $\begin{array}{l}\text { Retired (NHS nursing) } \\
\text { single divorced }\end{array}$ & $<£ 15 k$ \\
\hline $108 d$ & 68 (f) & $\begin{array}{l}\text { Retired (administration) } \\
\text { single divorced }\end{array}$ & $<£ 15 k$ \\
\hline $111 d$ & 67 (f) & $\begin{array}{l}\text { Retired (secretary) } \\
\text { Partner retired }\end{array}$ & $£ 25-50 k$ \\
\hline $117 d$ & 69 (f) & $\begin{array}{l}\text { Retired (University librarian) } \\
\text { re-married, husband retired }\end{array}$ & £15-25k \\
\hline
\end{tabular}




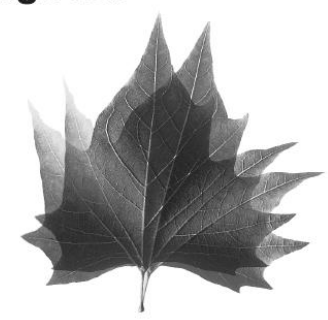

School of Environment and Technology

For $\mathrm{PhD}$ research on: 'Seaside town regeneration and the interconnections between the physical environment, key agencies and mid-life in-migrants.

- I agree to take part in this research which is to explore the connections of mid-life migration on seaside town's regeneration

- The researcher has explained to my satisfaction the purpose, principles and procedures of the study and the possible risks involved.

- I have read the information sheet and I understand the principles, procedures and possible risks involved.

- I am aware that I will be recorded during this meeting.

- I understand that the anonymised data will be used in Tony Leonard's $\mathrm{PhD}$ thesis, related academic papers and funding body reports, and that any confidential information will be seen only by Tony and his University supervisors and will not be revealed to anyone else.

- I understand the audio and document data files will be stored in a password protected computer file and then will be destroyed as confidential waste ten years after the completion of Tony's PhD.

- I understand that I am free to withdraw from the study at any time without giving a reason and without incurring consequences from doing so.

- I agree that should I withdraw from the study, the data collected up to that point may be used with my permission by the researcher for the purposes described in the information sheet.

Name (please print)

Signed

Date: 


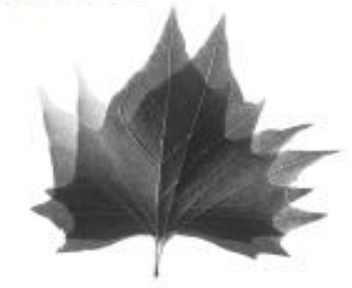

School of Environment and Technology

Cockcroft Building

Moulsecoomb

Brighton BN2 4GJ

Telephone 01273600900

Fax 01273642922

\section{Meetings Information Sheet for PhD research on:}

'Seaside town regeneration and the interconnections between the physical environment, key agencies and mid-life in-migrants'.

\section{Why have I been given this information sheet?}

High quality research requires participants be fully equipped with the information they need to make informed decisions about their involvement in the research process.

\section{What is this sheet?}

This information sheet explains:

- who I am and where I am studying,

- the nature and purpose of my research,

- my data collection process,

- how that data will be stored, used and presented,

- who to contact if you have any questions or concerns.

\section{Who am I?}

My name is Anthony (Tony) Leonard. I am a PhD student at the University of Brighton. My PhD is funded by Rother District Council. The research is entirely independent of Rother District Council. They have no access to the data I collect or influence over the nature of the research, or participants selected. They will receive an anonymised executive report of my findings only.

\section{What am I researching?}

The broad area of my study is 'mid-life migration to seaside town's' with Bexhillon-Sea as my case study. I am looking at the interconnections between the physical environment, the role and influence of key agencies and mid-life migration on seaside town's regeneration.

In order to try to understand these interconnections I need to examine the different migration motivations and decisions made by mid-life people. As part of this process I will be undertaking a number of in-depth interviews with mid-life people who have migrated to Bexhill-on-Sea in the last 5 years. 
What measures are taken to ensure the accuracy, security, anonymity and confidentiality of the data collected?

The most reliable way for me to ensure I accurately capture the information shared in meetings is to electronically record them and then transcribe the recordings. My two University academic supervisors and I are the only people with access to the raw data from the recordings. The data will be stored on a password protected personal computer and discs held in a secure location. The data from the recordings will be coded so as to ensure your anonymity in any presentation of the findings. The audio and document data files will be destroyed as confidential waste ten years after the completion of my $\mathrm{PhD}$.

What if you would like to view the research results?

As part of a process of communication of the research findings at the end of the project you will be given the opportunity to select to view the results of the research in your preferred format (i.e. hard copy executive report, poster display or presentation).

\section{How will this information/data be used?}

For the purposes of my research raw data includes verbal communication during the interviews. With the approval of respondents the anonymised data from this interview will be included in my $\mathrm{PhD}$ thesis, related academic papers and reports for the funding body If you wish to see the transcript of the interview I can provide this for you to check it is accurate.

\section{What if you don't want to be recorded?}

Please let me know if you would prefer not to be recorded and I can simply take notes. Alternatively, please email or phone me after the meeting (using the contact details below) and I can remove your comments from the records.

\section{What if you don't want to be involved or decide to withdraw?}

You can withdraw from taking part in the research at any time and you will be asked if you want your data to be removed from the records.

What if you have any questions or concerns?

Please feel free to ask me any questions and I will try to answer these as best I can. If you have any concerns with the research please contact Professor Callum Firth, Head of School, School of Environment and Technology at the University of Brighton on Tel: 012736422289.

For further information on this research please contact:

Tony Leonard

Tel: 07808572329

Email: A.R.Leonard@brighton.ac.uk

\section{Thank-you for taking the time to read this information and for any involvement in my research.}




\section{Appendix 13a}

\section{(a) Interview schedule - non-statutory agencies}

\section{e.g. Corporate Business}

Before commencing interview - prompt notes:

My name is Anthony (Tony) Leonard. I am a PhD student at the University of Brighton. My PhD is funded by Rother District Council. The research is entirely independent of Rother District Council. They have no access to the data I collect or influence over the nature of the research, or participants selected. They will receive an anonymised executive report of my findings only.

I attach two forms:

1. A meeting information sheet - that I will ask you to read

2. A participant consent form - that I will ask you to sign once you are satisfied with the information I have given you and understand the nature of my research and your role as participant

During the interview I will make notes as an aide memoir but the information will be recorded and transcribed at a later date. I will now start the interview with some background questions and then open up with some more detailed questions. I expect the interview to take about 45 minutes to an hour.

\section{Background Questions}

1. Name

2. Nature of business

3. Describe your position and role within the organisation

4. How long have you been with the company

\section{Business decisions and investments}

I would like to explore your stance on investments in seaside towns with a focus on Bexhill

5. How do companies like yours base their business investment decisions

a. what are the key criteria (demographic profiles, spend, catchments, ROI, etc)

\section{Prompt questions}

b. So what would be the constraints of an investment decision in Bexhill

c. What would be the tipping point between investing and not investing

6. How important is location to you as a business 
7. Organisations such as Commission of Architecture and Built Environment (CABE) have identified that food retailers are affecting regeneration within towns (both positive and negative) - what is your view of the retailers function?

8. Who do you feel are the key agencies that lead on regeneration and influence and drive changes within a town

9. What role do local authority regeneration and planning strategies have in your investment business decisions

\section{Prompt questions}

a. Do you actively look at planning and regeneration strategies to guide your investment decisions and what is it you look for

\section{Bexhill's present}

I would like to explore your thoughts on how your organisation would view Bexhill's current physical environment, its resident and migrant populations and the role of key agencies in affecting your investment decisions

10. How would you describe the current socio-economic performance of Bexhill

\section{Prompt questions}

a. What validates your analysis of Bexhill's economic performance

b. Is this considered good/bad by standards to other seaside town investments

c. How would you describe the towns physical environment

d. What is your view of the towns economic performance

e. What is your view of the population of Bexhill

f. What is the personality of the place

11. Do investment decisions take into consideration the physical environment of a place above that of the investment decisions you described earlier such as socio-economic criteria

12. What in your opinion are the interconnections between changes in the physical environment, actions by key agencies (such as large corporations and food retailers) and/or individuals to affect regeneration

13. Do you feel it is within your organisations power to act as a regeneration catalyst

\section{Prompt questions}

a. should it be your role

b. if so, how do you affect regeneration

14. Does the Council contribute/constrain your ability to invest or are there more overriding factors 


\section{Prompt questions}

a. What are the constraining factors

b. Does this affect your investment strategies

c. Do businesses, such as yours, believe in partnership working with local authorities or is it purely 'bottom-line' profits that drive decisions (ROI/NPV)

15. As a business trading in Bexhill what do you feel are the main advantages and what are the main disadvantages of trading here

\section{Bexhill's future}

16. What is your company's view and scenario planning on the future of Bexhill and your investment strategy decisions

\section{Prompt questions}

a. How do you think your organisation can contribute to change in Bexhill?

17. Regeneration is a key goal of the Council but where do you believe the future regeneration drive for Bexhill will come from

\section{Prompt questions}

a. Who do you see as being the key agencies for change within the town

b. Where does the power and influence actually lie to make the changes within the town

18. What drives regeneration and your investment decisions

\section{Prompt questions}

a. changes in the physical environment

b. changes in nature and character of the people

c. changes in the image and personality of a place

d. changes in rental values

19. What drives regeneration - changes in the physical environment or the nature and character of the people living in or migrating to the place 


\section{Appendix 13b}

\section{(b) Interview schedule - non statutory agencies}

\section{e.g. Chamber and SME's}

Before commencing interview - prompt notes:

My name is Anthony (Tony) Leonard. I am a PhD student at the University of Brighton. My PhD is funded by Rother District Council. The research is entirely independent of Rother District Council. They have no access to the data I collect or influence over the nature of the research, or participants selected. They will receive an anonymised executive report of my findings only.

I attach two forms:

1. A meeting information sheet - that I will ask you to read

2. A participant consent form - that I will ask you to sign once you are satisfied with the information I have given you and understand the nature of my research and your role as participant

During the interview I will make notes as an aide memoir but the information will be recorded and transcribed at a later date. I will now start the interview with some background questions and then open up with some more detailed questions. I expect the interview to take about 45 minutes to an hour.

\section{Background Questions}

1. Name

2. Nature of business

3. Describe your position and role within the organisation

4. How long has the business existed in Bexhill

5. Total number of employees (FTE)

\section{Business performance in Bexhill}

I would like to explore your investment decisions in Bexhill

6. How do businesses like yours base your investment decisions

a. what made you choose Bexhill and why your type of business (demographic profiles, spend, catchments, ROI, gap in the market, etc)

7. Now that you are trading in Bexhill what do you now see as opportunity and constraints of an investment decision in Bexhill.

8. How important is location to you as a business 
9. What role have local authority regeneration strategies played in your business decisions.

\section{Bexhill's past}

I would like to explore your understanding and view of how Bexhill's past has come to determine its present day position

10. What do you see were the key influences on Bexhill's past that has shaped the town's current environment

11. Who do you feel were the key agencies and individuals that influenced how the town is presented today

12. What role has the planning authority and planning strategy played in the more recent development of the town

13. Describe what you perceive to be the personality of Bexhill as it has evolved both in its physical environment and its people and image

14. How has Bexhill been shaped to what it is today - through its physical environment, its population or its image

\section{Bexhill's present}

I would like to explore your thoughts on how you, as a small business in the town, view Bexhill's current physical environment, its resident and migrant populations and the role of key agencies in affecting change.

15. How would you describe the current socio-economic performance (personality) of Bexhill

\section{Prompt questions}

a. What validates your analysis of Bexhill's economic performance

b. Is this considered good/bad by standards to other seaside town investments

c. How would you describe the towns physical environment

d. What is your view of the towns economic performance

e. What is your view of the population of Bexhill

f. What is the personality of the place

16. Does the town need regenerating and if so is the town up for change and regeneration

17. How would you describe the current state of business in Bexhill

18. Is this what you expected when you first started your business

19. Did your business decision consider the physical environment of a place above that of the socio-economic make-up or visa versa before investing 
20. How has the Council contributed to this performance through past and present decisions or actions

21. How has the SME's contributed to this performance through past and present decisions or actions

22. What are the main opportunities and constraints to future investments in Bexhill

23. What do you feel has worked best and what has worked least well for Bexhill

24. What in your opinion are the interconnections between changes in the physical environment, actions by key agencies (such as the Council or large corporations and food retailers) and/or individuals to affect regeneration

25. Do you feel it is within your organisations power to act as a regeneration catalyst

\section{Prompt questions}

a. should it be your role

b. if so, how do you affect regeneration

\section{Bexhill's future}

26. How do you think your organisation can affect change in Bexhill?

27. If regeneration is a key goal of the Council where do you believe the future regeneration change for Bexhill will be driven from

28. Who do you see as being the key agencies for change within the town

29. Where does the power and influence actually lie to make the changes within the town

30. What role is there for SME's

31. Does planning strategy constrain or manage regeneration and what does it manage.

32. What drives regeneration - changes in the physical environment or the nature and character of the people living in or migrating to the place 


\section{(c) Interview schedule - statutory agencies}

Before commencing interview - prompt notes:

My name is Tony Leonard. I am a PhD student at the University of Brighton. My PhD is part-funded by Rother District Council. The research is entirely independent of Rother District Council. They have no access to the data I collect or influence over the nature of the research, or participants selected. They will receive an anonymised executive report of my findings only.

I attach two forms:

1. A meeting information sheet - that I will ask you to read

2. A participant consent form - that I will ask you to sign once you are satisfied with the information I have given you and understand the nature of my research and your role as participant

During the interview I will make notes as an aide memoir but the information will be recorded and transcribed at a later date. The interview will start with some background questions and then open up with some more detailed questions. I expect the interview to take about 45 minutes to an hour. I emphasis there are no right or wrong answers I just want to hear your views.

\section{Background Questions}

1. Name

2. Organisation representing

3. Position and role within the organisation

4. How long have you been working with the Council

Bexhill's present (explore views on Bexhill's socio-economic performance)

1. How would you describe and characterise Bexhill as it is today

a. How would you describe Bexhill's personality

b. How would you describe Bexhill's built environment

c. How would you describe the towns economic performance

d. How would you describe Bexhill's social structure

2. What influence does the character of Bexhill have on attracting people to live in or visit Bexhill

3. What, in your opinion, drives regeneration (does this apply to Bexhill)

4. What are the blockages to regeneration in Bexhill

a. How do the residents view change

b. How does the physical environment restrict change

c. How does the image play its part 
Bexhill's past (I would like to explore your views of Bexhill's past)

5. In the decades gone by what do you consider were the key influences that have shaped Bexhill and given it it's character

a. How influential has migration of people into the town been

b. How influential has the built environment been

6. How has the Council influenced economic and social change
a. Planning (housing/shops)
b. politics
a. Regeneration
d. promoting status quo
b. Were their actions strategic/intervening or responsive

7. What regeneration actions have worked best and what has worked least well for Bexhill

Bexhill's future (explore what Bexhill's future could look like)

8. How would you like to see Bexhill-on-Sea look in the future

9. Who and what do you see driving this change

a. What role will the statutory agencies play (Council's)

b. What role will non-statutory agencies play (Retirement homes/SME's/ retailers)

c. What role will the town's Leaders play

10. How do you see future residents and migrants shaping the socioeconomic performance of the town

a. What migration into the town will be important and why

11. What do you believe needs to adapt first to bring about changes in the socio-economic performance of Bexhill

a. changes in the physical environment

b. changes in the people (the nature and character of the people living in or migrating to a place)

c. changes in the 'real' or 'perceived' personality of the place 


\section{Appendix 14}

\section{Interview Schedule - in-migrant middle-lifers}

My name is Tony Leonard. I am a PhD student at the University of Brighton. My $\mathrm{PhD}$ is funded by Rother District Council. The research is entirely independent of Rother District Council. They have no access to the data I collect or influence over the nature of the research, or participants selected. They will receive an anonymised executive report of my findings only.

I attach two forms:

3. A meeting information sheet - that I will ask you to read

4. A participant consent form - that I will ask you to sign once you are satisfied with the information I have given you and understand the nature of my research and your role as participant

During the interview I will make some notes as an aide memoir but the information will be recorded and transcribed at a later date. The interview will start with some background questions and then recorded with some more detailed questions. I expect the interview to take about 45 minutes to an hour. I emphasis there are no right or wrong answers I just want to hear your views.

Explore reasons for migrating (moving) to Bexhill (A bit about them)

1. Could you provide a brief potted history of where you have lived

a. If appropriate, what were your main reasons for these moves

2. How did you know of Bexhill before moving and what were your perceptions

a. Past visits (purpose and occasions)

b. What was your view of the towns physical environment then

\section{Personality and people of Bexhill}

3. Why did you choose Bexhill and did you consider anywhere else

a. What were your reasons for moving to Bexhill (downsizing, divorce?)

b. What attracted you most to live in Bexhill

c. Where do key family and friends live - did they influence your decision

d. To what extent did you take account of the people who lived in Bexhill

e. Do you feel the people have similar traits/characteristics to you

f. What are the similarities and dissimilarities between you and them

4. Will Bexhill be your permanent home or will you move again in the future

5. What is/was your main profession and what are you currently doing in Bexhill

a. Retired b. In-between jobs c. Home-maker d. Carer

e. Employed (what is the nature of your work and where do you work) 
f. What links have you through work to other places

6. Do you believe you be earning/working beyond retirement age and why

a. If so how and where will you earn/work

b. Does Bexhill offer that opportunity to earn a living/supplement pension

Bexhill's present (explore how they see Bexhill's socio-economic structure)

7. Now you are living here how would you describe and characterise Bexhill

a. How would you describe Bexhill's personality

b. How would you describe Bexhill's built environment

c. How would you describe Bexhill's economic performance

d. How would you describe Bexhill's social structure

8. What is it that you like best about Bexhill and what do you like least
a. Town centre
d. Street spaces
a. Seafront
e. Parks
b. Suburbs

2. How do you balance the dislikes with wanting to live here

3. Where do you believe Bexhill could be improved

4. So what needs to change to make Bexhill a better place for you to live

5. If regeneration is necessary what are the problems that prevents it

\section{Bexhill's Future}

6. How do you see Bexhill changing and how do you see this impacting on you

a. How do you see it changing

b. what do you see as the causes of the changes

c. if no change, then why no change

Explore
d. Town centre
g. Street spaces
e. Seafront
h. Parks
f. suburbs

7. Who and what do you see driving the future of the town
a. What role will the Council's play
b. What role will businesses play (Retirement homes/SME's/ retailers)
c. What role will the town's Leaders play
d. What role will residents play

8. What needs adapting first to bring about changes in Bexhill and what might be the constraints that prevent the adaptation

d. changes in the physical environment

e. changes in the people (nature/character of the people living/migrating) 
f. changes in the 'real' or 'perceived' personality of the place

\section{Background questions (not for recording)}

9. How many and make-up of the household

10. Relationship with those in the household

11. Type of property living in

a. Owned - with or without a mortgage?

b. Rented-social housing or private?

c. Type of property

i. Bungalow, semi-detached, detached

ii. Approx age of property

12. Total household income per annum
a. Less than $£ 15 \mathrm{k}$
b. $£ 15 k-£ 25 k$
c. $£ 25 \mathrm{k}-£ 50 \mathrm{k}$
d. $+£ 50 k$ 


\section{UNIVERSITY OF BRIGHTON \\ SCHOOL OF ENVIRONMENT AND TECHNOLOGY ETHICS APPROVAL FORM MPhil/PhD and STAFF RESEARCH PROJECTS}

This form is to be used by MPhil/PhD students and staff seeking ethical approval for their research from the School of Environment and Technology Research Ethics and Governance Committee.

All of those completing this form and must receive approval from an appropriate ethics committee (usually the School of Environment and Technology Research Ethics and Governance Committee) prior to commencing their research.

Please read the University Guidance on Good Practice in Research Ethics and Governance before completing this form. This form should be checked carefully for typographical and grammatical errors before submission. Incomplete or badly presented forms will be returned. Supervisors of student projects have a responsibility to ensure that the guidelines are followed and that applications are properly presented.

If after considering this form the School Research Ethics and Governance Committee consider Tier Two approval is required, the Principal Investigator / Research student will be notified and this form automatically passed to the Chair of the Faculty Research Ethics and Governance Committee for consideration.

\section{Please attach the SET Research Ethics Checklist you have already completed to this form.}

\section{Section A - Key details}

1. Name of student/Principal Investigator

Anthony Leonard

2. Name of supervisor (for MPhil/PhD students)

Professor Andrew Church

Professor Neil Ravenscroft

3. Title of project (no more than 20 words)

'Seaside town regeneration and the interconnections between the physical environment, key agencies and mid-life in-migrants'. 


\section{Aims of the study}

Please summarise your aims in one or two sentences. Write no more than 100 words.

The overall aim of the research is to examine the processes of change in a seaside town using a Lamarckian perspective. The thesis will aim to understand the effect on development in a seaside town of the interconnections between changes in the physical environment, actions by key agencies and individuals and the nature of migration, especially the role of mid-life in-migrants (50-70 years of age).

\section{Research context}

A brief summary should be provided discussing the relevant published literature so that the Committee can understand the context to your research. In addition, please supply four or five up-to-date references to the relevant published literature. You may supply up to 800 words.

This summary frames the research question that emerged through the literature review in the regeneration of seaside towns and in particular the possible relationships between the physical environment and the human capital. The literature review drew on three different bodies of work concerned with the processes of seaside urban regeneration, the influence of key agencies and the nature of in-migration to seaside towns.

As seaside resorts' fortunes changed in the 1960s and 1970s many of the towns grew in population through in-migration of those commuting; the relocation of benefit claimants; and, particularly, retirement, that took over from the holiday industry and that has changed the structure of some resorts (Walton and Browne, 2010). The research literature also identifies these structural changes and how certain agencies are able to influence the external environment (knowingly and unknowingly) and in turn affect the human capital both visiting and living in the seaside town (Agarwal, 2007). The human capital then has an influence on the 'personality' of a place. With cheaper, poorer conditioned housing attracting a perceived, and real, in-migration of the less well-off and economically inactive ageing population (Beatty et al., 2008) this, amongst other things, contributed to the changing fortunes and structural changes of many seaside resorts (Benedict and Houghton, 2009).

The literature review argues that a Lamarckian perspective can help understand these changes by seeing them as being the result of a complex series of interactions between an external environment, the planning system and development decisions by key agencies, rather than being an outcome of an evolutionary process as suggested by models of resort development (Lamarck, 1809; Ravenscroft and Hadjihambi, 2006). By intervening in the external environment this stagnation and decline in seaside towns has in some cases altered into new growth. Therefore, the ability of key agencies involved in seaside regeneration to develop a series of improvements to the built environment that allows it to adapt and alter the human capital is a critical focus for examination in changing resorts (Russell and Faulkner, 2004; Tallon, 2010). Previous research has shown the impact that retirement in-migration has had on many resorts. Currently, however, the in-migrant mid-lifers' (people who are 
aged 50-70 who are pre-retirement and retirement ages) have motivations that may be different to those of past retirees as 'lifestyle', 'means' and 'amenity' migration may be intertwined to support their accustomed lifestyles (Benson and O'Reilly, 2009; ELSA, 2008). Many in-migrant mid-lifers are wealthier and healthier than ever before and lead a consumptive lifestyle (Barnes et al., 2004) that may have implications for seaside towns. This cohort could be influenced by key agencies 'knowingly' adapting the external environment through urban management measures. However, understanding what influences the migration patterns of mid-lifers requires further research into their migration motivations and the motivations of other social groups that also migrate to seaside resorts.

Migration decisions involving resorts are partly influenced by the 'personality' and social characteristics of particular places. Thus the longer-run issues facing these resorts have as much to do with, their 'psycho-social environment' (Florida, 2008), as to do with their business climate and economic structure. Therefore, migration patterns, especially of mid-lifers may shape a resort but, importantly, it appears migration choices may be shaped by their perceptions of place, the housing, employment and consumption opportunities, amenities and aesthetic and psycho-environment to meet their demands and expectations as they move into a new period of their life cycle stage. Such mobility flows have shaped and continue to reshape the social ecology of seaside resort towns (Miles, 2005; Florida, 2010).

The research literature indicates that as the external environment changes so the in-migration of populations that is attracted to this changing environment, influence the character and personality of the seaside town and in turn the socio-economic performance. Therefore, three aspects of further research are required; firstly, is a better understanding of motivations of in-migrants to resorts, especially mid-lifers; secondly, identifying the role of key agencies in changing the external environment; and thirdly, how changes in the external environment affect migration and wider social and economic changes in seaside towns.

Based on the above discussion the following summarises the rationale for the research:

Firstly, the Lamarckian perspective, that by changing the external environment, should, in theory, cause changes in the human capital living in and visiting that environment, that in turn causes changes in the economy and 'personality' of the seaside resort and its consequent regeneration or degeneration. This perspective requires an examination of these connections between the physical environment, in-migration especially of mid-lifers and the policies of key agencies.

Secondly, retirement will be much more complex due to the consumption and work lifestyles of mid-lifers and requires further examination in the context of migration to seaside towns.

Thirdly, retirement may increasingly involve 'income generation', and it may be important for in-migrants, especially mid-lifers, to remain in, or migrate to, the region in which they have good employment-related connections. A resort on the south east coast of England where migration has been a major influence on 
economic and social change but is also accessible to work, leisure amenities and consumption activities in a wider region is where this research will focus.

\section{Research design}

Please provide no more than 800 words and ensure that you discuss your sampling strategy (if appropriate), data collection methods and strategy for data analysis.

\section{Case Study: Bexhill-on-Sea}

Bexhill-on-Sea is considered a suitable case study in that it is located in the south east of England and is a location where socio-economic change has been strongly shaped through the in-migration of the retired. The complexity of key agencies influencing (knowingly and unknowingly) the external environment has had profound implications on the town's retirement population; but with the recent increased emphasis on regeneration in the town and with 'retirement' migration especially of mid-lifers becoming much more complex this makes Bexhill-on-Sea an appropriate case study. The complexities involved in resort change are present in this single case study and, therefore, is believed an effective research location for understanding these complex social phenomena.

The primary data collection methods will include; i) semi-structured in-depth interviews with the key agencies involved in shaping the external environment through planning and regeneration; ii) in-depth interviews with the mid-life and retiree in-migrants who have moved into Bexhill-on-Sea in the past five years. The purpose of the interviews with key agencies is to understand the context in which they currently operate. How these formal actors consider their role in the future socio-economic dimension of regeneration and how they believe they impact upon this. The interviews with in-migrant mid-lifers and retirees aim to understand their motivations for migration to a seaside resort and whether the external environment or the 'personality' of the place has had an influence on their motivations. These interviews will be piloted by doing 2 or 3 interviews, reviewing and deciding if the processes and questions need modifying. Attached at (Appendix 8a, 8b) are some draft interview schedules.

The sampling strategy includes two approaches; i) a purposive sampling technique in the selection of key agencies and stakeholders for interviews. These have been identified through the existing governance and trading structures and access will be facilitated through the Council. They include representative of both formal statutory organisations (e.g. council) and nonstatutory private and third sector organisations (e.g. chamber of commerce); ii) the sampling of the in-migrant mid-lifers (50-70 age category) who have moved to the town within the past five years will be undertaken by contacting individuals registered on the citizen panel and through promoting (by invitation to participate) in the District wide newsletter (see Appendix 6 but note advert is draft as newsletter editors may wish to make adjustments). The researcher has approval by the Council to use these approaches (Appendix 7).

The interviews will be conducted using digital recordings (subject to informed consent); transcription and a research process audit trail (use of electronic diary and electronic filing of all communications and research plans). This data will be stored on password protected computer, computer files and encrypted USB 
sticks. Any hard copies will be stored in a secure location with researcher access only. The ethical checks will be via the ethics panel procedure, respondent validation, informed consent and the coding of meetings and interview data to ensure anonymity. To ensure validation and robustness of the research the data will be analysed using techniques and practical procedures such as constant comparative analysis, reflective notes, respondent validation, triangulation of source and methods, and a clear audit trial and use analysis processes that involve 'coding' or 'indexing' (Strauss and Corbin, 2008)

The justification of the methodology and research design is drawn from the research question. For the purposes of this research, theorisations influenced by Lamarckian research are adapted to understanding the effect on development of the interconnections between change in the physical environment, actions by key agencies and individuals and the changing nature of migration to the seaside resort. The interview process will be a prominent method for gathering an understanding of the epistemological issues of contextuality, criticality, contingency and complexity (Silverman, 2009).

Much of the data gathering will be the collection of a set of human experiences through the construction and reconstruction of personal stories and is well suited to addressing issues of complexity and human centeredness because of its capacity to record and retell those events that have been of most influence on them and is based on the respondent's life experiences and entails chosen parts of their lives.

With regards to self-awareness and reflective practice, the researcher is employed and sponsored by the local authority in which the case study is being undertaken. Bias concerning the position of the researcher in the research process is, therefore, recognised as a crucial issue. And yet, Jacobs (1993), in 'The Death and Life of Great American Cities' emphasised the importance of emersion and being in context as instrumental in conducting successful research. The research design will address this potential bias through respondent validation, reflective notes and a clear audit trial.

7. Provide details of financial sponsorship and any ethical issues this may raise (50-150 words)

Rother District Council is the sponsor of my research and I am employed by them as a Director, accountable for a number of services that touch upon the research questions (i.e. regeneration, planning, housing and amenities). However, the work I am doing is entirely independent and Rother District Council have not influenced the specific nature of my aims or objectives.

Their role as a key agent in my case study is acknowledged and, therefore, I will employ reflective practice to try and make explicit any ethical issues that arise from my relationship.

The key ethical issue for me, however, is communicating my relationship to research participants, some of whom I may be familiar with through my work. I will address this by being honest and explicit about the sponsorship and also the independence of the work in any communication or contact. I will also ensure I have high quality documentation for all interviewees concerning the 
nature of the project.

There are no ethical issues that arise from using the citizen panel or Council newsletter as all participants have given their consent to be contacted for further research purposes (Appendix 7).

8. If the project involves funding from a Research Council or other organisation with an ethics policy (e.g. a charity) please confirm that the organisation's ethical procedures have been considered and outline any actions taken.

Rother District Council has the following in place in which the Researcher is expected to abide by. There are no actions necessary other than being aware and compliant with the policies.

- Dignity at work (Appendix 4)

- Single Equalities Scheme 2010-2016 (Appendix 5)

Please use the SET Research Ethics Checklist to decide which additional section(s) of this form to complete and complete appropriately

If you ticked yes to Question 1 in the checklist (Negative Environmental impacts) complete Section B

If you ticked yes to any of Questions 2-9 (Human Participant Issues) complete Section C

If you ticked yes to Question 10 (Indirect Involvement of the Public) complete Section D

If you ticked yes to Question 11 (Secondary Data Sources) complete Section E The project student and the supervisor or the principal investigator in the case of staff research must sign the form in Section $F$ 


\section{Section B - Potential Risk to the Environment}

The aim of this section is to check whether you have taken the necessary steps to ensure your research will avoid causing significant negative impact on the environment.

9. If the research is likely to have significant negative impacts on the environment provide details of these impacts (for example the release of dangerous substances or damaging intrusions into protected habitats).

$\mathrm{N} / \mathrm{A}$

10. Please describe how you will mitigate against significant environmental harm and manage risks.

$\mathrm{N} / \mathrm{A}$

\section{Section C - Potential Risk to Human Participants directly working with the researcher}

The aim of this section is to check whether you have taken the necessary steps to ensure your research will avoid causing physical or emotional harm, pain, discomfort or stress to human participants.

11. If human participants are directly involved provide brief details regarding the participants and how they will be contacted (e.g. number, age, gender, ethnicity, general residential location).

There will be 3 groupings of participant involvement in this research. These are the key agencies that make up the statutory and non-statutory actors and the inmigrant mid-lifers who have volunteered to partake. Examples of the statutory and non-statutory organisations are given below and the procedures that will be used to contact these agencies.

Examples of the formal statutory sector actors include the Leader of Rother District Council and Senior Officers at both Rother District Council and East Sussex County Council. Interviews for statutory sector actors will be arranged by myself and normally undertaken in their offices, unless they request another venue, where every effort will be taken that the interview is not overheard.

Examples of the third sector and private business actors will include three major corporate businesses within Bexhill (Sainsbury, Land Securities and Hastings Direct Insurance company). It is anticipated that the researcher will gain access to the Regional Development Directors of Sainsbury and Land Securities having previously had business connections at this level. The Managing Director for Hastings Direct will be contactable through my work connections. The interviews will be held in person at their place of work, unless they request another venue, at a convenient time and agreeable timescale. Examples of the third sector actors to be interviewed include the De La Warr Pavilion Trust Director and the same principles will apply as to the corporate businesses. 
Other examples of non-statutory sectors are the Chamber of Commerce and Federation of Small Businesses. All participants will be contacted in advance to gain consent and provided with the research information sheet. They will be contacted via the communication channel most suited to them. This process of obtaining consent and providing information will be repeated verbally in the interview recording to ensure understanding, opportunity for questions and peace of mind for the participants.

Interviews with in-migrant mid-lifers will by way of invitation and conducted in their place of residence. The invitation will come through either a mail-shot to the citizen panel (the councils list of residents who have willing agreed to partake in research and consultation activity) or an invitation to participate in a section of the Rother District-wide newsletter. The Council have agreed to allow access to these mediums and this does not breach any of their data protection policies (Appendix 7). It is intended to undertake 20-30 in-depth interviews between the age ranges of 50 to 70 years. These will be separated into four groupings $(50-54 ; 55-59 ; 60-64 ; 64-69)$ and five will be drawn from each group.

It should be noted no vulnerable participants will be involved in the research with the key agencies. However, whilst the in-migrant mid-lifers will all be adults, aged 50-70 years of age, the researcher cannot control those who may come forward and who may be of a vulnerable nature. The researcher has 15 years of working in housing and community services and does have an understanding of such groups and has previously undertaken equalities and vulnerable group training. If it is felt that the interview may not be appropriate with any vulnerable person or group then the researcher will retire from the interview in a tactful manner. A further participant will then be chosen as outlined above. Each participant will be contacted in advance to gain consent and provided with the research information sheet. They will be contacted via the communication channel most suited to them. This process of obtaining consent and providing information will be repeated verbally at the interview and at the point of recording to ensure understanding, opportunity for questions and peace of mind for the participants. Interviews will normally take place at the participant's place of residence, unless they request another venue. Before entering the participant's place of residence the researcher will provide an identity card verifying his authenticity. All information sheets will make clear that the research is being done for $\mathrm{PhD}$.

Depending on the nature of response to my request for participant involvement the following procedures are in place: If some key agencies or in-migrant midlifers refuse me access to an interview then I will need to reflect on this; if they do not want to be recorded then I will take written notes.

12. If human participants are directly involved provide details of any participants who might be considered vulnerable due to age or to a social, psychological or medical condition. Examples include children, people with learning disabilities or mental health problems but participants who may be vulnerable are not confined to these groups (see the University's 'Guidance on Good Practice in Research Ethics and Governance' for more details. Proposals involving such 
participants are often likely to require ethical approval from the Faculty of Science \& Engineering Research Ethics and Governance Committee).

It is unlikely that vulnerable people will be interviewed as the sampling will be self-selecting through the citizen panel or through the invitation via the Districtwide newsletter. It is not anticipated that these vulnerable groups will come forward. If, however, it becomes apparent vulnerable groups are involved then proper procedures will be followed as set out in section 13.

13. If human participants are directly involved provide details of any risks participants are likely to face that would not be considered minimal risks (see the University's 'Guidance on Good Practice in Research Ethics and Governance' for details of possible risks including, but not limited to, physical risks to participants, distress arising from prolonged testing or questions of a sensitive nature, risks for researchers and risks for vulnerable people).

If risks are only minimal please describe the risks and explain why you believe they are only minimal.

Only minimal risks are considered as the interview questions are more to do with their migration motivations to the seaside town.

However, there may be occasions during the course of the research, when I may be engaging with participants who have undergone harrowing or emotionally disturbing experiences that may cause the participant embarrassment, discomfort or grief. Examples of such experience might include moving to the seaside town because of illness and other life difficulties. This may raise issues with the interviewer and interviewee and, therefore, if the participant becomes distressed they will be given the opportunity for the audio tape to be switched off, or take a break or stop the interview entirely.

Of particular concern is if the researcher comes across an illegal activity then I will follow the University guidance on disclosure.

There is also a recognised risk to the researcher going into a person's home. I intend to complete the University risk assessment form and will always advise someone where I am going, report when I have finished and keep my mobile phone turned on, in 'silent' mode.

If I become perturbed or upset by stories I may hear I have the advantage of using my employers staff support scheme to discuss it with them but not to disclose any details that could identify the respondent. 
14. Describe the procedures that will be put in place to ensure safe and ethical direct involvement of human participants (Where necessary and as appropriate include comments where necessary on obtaining informed consent, reducing harm, providing feedback and accessing participants through an individual providing information such as a teacher, manager, employer etc.). Examples of consent and information forms can be found on StudentCentral.

A key ethical consideration is generated by the sensitive nature of the data (due to business confidentiality, a political dimension or in-migrant mid-lifers personal statements) that will be provided on occasions in confidence by participants. However, all participants will be adults over 21 and some will be experienced professional individuals in their roles. For each group the following procedures will be employed to ensure their safe and ethical direct involvement in my research.

Prior to each interview I will ensure the participants receive the consent forms and information sheets with a covering note (Appendices 2, 3a and 3b) explaining the voluntary nature of involvement in the research; their right to withdraw from the research at any time; their anonymity as a participant through coding of names; the security of data held through secure data management process; and in the case of interviews the opportunity for respondent validation. This will be sent via email or a hard copy by post depending on participant access to email. I will repeat the key points below verbally before each interview. The consent form will be signed by the participant and retained by the researcher. The information sheet will be taken away by the participant. Where participants have not had adequate time to provide consent they will be given a month to contact me via email, post or telephone to withdraw if they wish. Participants will be informed that they can of course withdraw at any point after giving consent without any consequences. If they do not want to be recorded then I will take written notes only. Interviews will be set up by email or telephone, and the interviews will be mostly held in person in the participant's place of work, or place of residence, at a convenient time and agreeable timescale for them. Every effort will be made to ensure that the interview cannot be overheard.

The purpose of the consent forms and information sheets and reconfirming these points at the time of the interview is to ensure that there is a clear verbal and written explanation of research aims, objectives and methods and the reason, value and extent of the participant involvement. It will make clear the contact details for the researcher and head of school if participants have any questions or concerns. In advance of the research process I will allow sufficient time for participants to ask the researcher questions and following the interviews I will offer all participants the opportunity to engage in a respondent validation meeting to ensure they are happy with the transcripts.

In the final stage of my research project I will communicate my findings and this may take the form of a Q\&A session following a brief presentation at meetings, or formal short paper written for distribution, whichever medium and format is preferred by the respective groups. This strategy will be supplemented by formal academic dissemination at conferences, seminars and in publications. 
15. If covert or other controversial research methods are to be used or if the research procedures contravene conventional ethical protocols (including consent, confidentiality and feedback), justify the use of such methods and procedures here and outline the measures that will be put into place to mitigate against potential harm. If no controversial techniques will be used and the research will follow normal ethical protocols, please write 'normal ethical protocols' in the box below.

Normal ethical protocols

16. If human participants are to receive financial reimbursement for their time (excluding reasonable expenses to cover travel and other costs) provide details and a short justification (e.g. amounts and form of reimbursement).

$\mathrm{N} / \mathrm{A}$

17. Describe in $50-100$ words how you will ensure data collection is confidential and anonymous (e.g. interviews cannot be overheard, details will not be accessible to others), how data will be stored and who will have access to the data. If the data will not be confidential or anonymous outline the justification for this decision here and procedures for mitigating against potential harm. In particular, please outline consent and data protection procedures for the use of participants' images if photographic or video recordings are to be made in the course of the research.

All data should be stored securely. Documentation should be kept in a locked cabinet or desk, and electronic data should preferably be kept on a removable disk or data stick which can be locked away, or if this is not possible on a password protected computer. (see the University's Guidance on Good Practice in Research Ethics and Governance for further details)

For all interviews and focus groups I will use digital recording to improve accuracy of transcription. However, where participants request manual recording this will be done in a research diary.

Transcription of the interviews will only be by the researcher and validated by the respondent if they wish to do so. All transcriptions will be coded (names of participants and organisations) in order to ensure confidentiality and anonymity.

Analysis will be by manual discourse analysis with use of spreadsheets

The storage and audit trail of all research diaries and electronic communications (audio and transcriptions) will be either stored on a password protected computer and backed up on encrypted USB sticks; or filed in a lockable desk accessible only by the researcher. Only the researcher and my two supervisors will have access to this raw data. These files will be archived for ten years after the completion of my PhD and thereafter disposed as confidential waste. 


\section{Section D - Potential risk to members of the public indirectly involved in the research without their knowledge at the time}

The aim of this section is to check whether you have addressed any ethical issues arising from activities such as covert observation of people in non-public places and the use of methods that will affect privacy.

18. If the public are indirectly involved in the research without their knowledge at the time please provide brief details (e.g. how they will be involved and (where known) the age, gender, ethnicity and location of those who will be indirectly involved).

N/A

19. Provide details of any negative impacts members of the public will be likely to face and that would not be considered minimal impacts (e.g. invasion of privacy, harm to property, being subject to what an individual perceives to be inappropriate behaviour). If risks are only minimal please describe the risks and explain why you believe they are only minimal.

$\mathrm{N} / \mathrm{A}$

20. Describe any procedures that will be put in place to ensure safe and ethical indirect involvement of members of the public (include comments where necessary on providing information and feedback if requested by the public). Examples of information forms can be found on StudentCentral.

$\mathrm{N} / \mathrm{A}$

21. If covert or other controversial research methods are to be used or if the research procedures contravene conventional ethical protocols (including consent, confidentiality and feedback), justify the use of such methods or procedures here and outline the measures that will be put into place to mitigate against potential harm. If no controversial techniques will be used and the research will follow normal ethical protocols, please write 'normal ethical protocols' in the box below.

$\mathrm{N} / \mathrm{A}$

22. Describe in 50-100 words how you will ensure data collection is confidential and anonymous (e.g. people will not be able to be identified by photographs or notes taken by observers), how data will be stored and who will have access to the data. If the data will not be confidential or anonymous outline the justification for this decision here and procedures for mitigating against potential harm.

All data should be stored securely. Documentation should be kept in a locked cabinet or desk, and electronic data should preferably be kept on a removable disk or data stick which can be locked away, or if this is not possible on a password protected computer. For undergraduate projects normally only the 
student and supervisor will have access to the data (see the University's 'Guidance on Good Practice in Research Ethics and Governance' for further details).

\section{N/A}

\section{Section E - Secondary Data}

Secondary data refers to any data you plan to use that you will not collect yourself. Examples of sensitive secondary data include datasets held by organisations, patient records, confidential minutes of meetings, and personal diary entries (these are only examples and are not an exhaustive list)

23. Please provide details (50-100 words) regarding any secondary data to be used that may carry sensitive personal or sensitive organisational information.

The secondary data will be publicly available (e.g. in libraries, town halls, public body archives and on organisation websites) and as such will not contain sensitive or private data.

If there are documents considered sensitive or contain private or confidential data the procedures in section 24 will be followed

24. If secondary data sets containing sensitive personal or sensitive organisational information are to be used outline how such use will be ethically managed (include details such as anonymising data sets, ensuring protection of source agency, gaining consent of data owners, and how the data will be stored).

Where documents are private (i.e. not publicly available, such as Cabinet reports) I will gain the consent of the authors to use and store that data ethically, and ensure it is coded to guarantee confidentiality and anonymity (including names of participants and where relevant organisations given generic umbrella terms).

The storage of, and access to, this data will follow the same procedures as outlined in section 17.

\section{Section F - Further Details, Accompanying Documentation and Signature}

25. Please add anything relating to ethical issues that should be considered when assessing this project that has not been addressed elsewhere on this form. Continue on another sheet if necessary.

N/A

26. Indicate which of the following are attached to this form. 
The Research Ethics Checklist should be attached for all projects; you only need to provide the other documents if they are applicable to your project

Attached

SET research Ethics Checklist (please remember to attach) - Appendix 1

Participant information sheet - Appendix 2

Participant consent form - Appendices 3a, 3b

Dignity at Work - Appendix 4

Equalities scheme - Appendix 5

Material to be used to advertise the project - Appendix 6

Approval from Rother District Council - Appendix 7

Draft interview schedules - Appendices 8a, 8b

Bibliography - Appendix 9

Please note that projects that use questionnaires to be completed by respondents do not need a separate consent from, as consent is inferred if the questionnaire is completed; however, the opening statement on the questionnaire should indicate that this will be the case. All those completing a questionnaire should be offered an information sheet providing further details of the project and contact details of the University. When questionnaires are conducted by the researcher as part of an interview then a consent form should be signed.

27. Please sign this form.

Student / Principal researcher's name

Signed

Date

28. This form must be checked and approved by your supervisor (for MPhil / PhD students)

Any further Comments from supervisor:

Supervisor name

Signed

Date 


\section{Appendix 16}

16a. Population projection for Bexhill-on-Sea for 2031

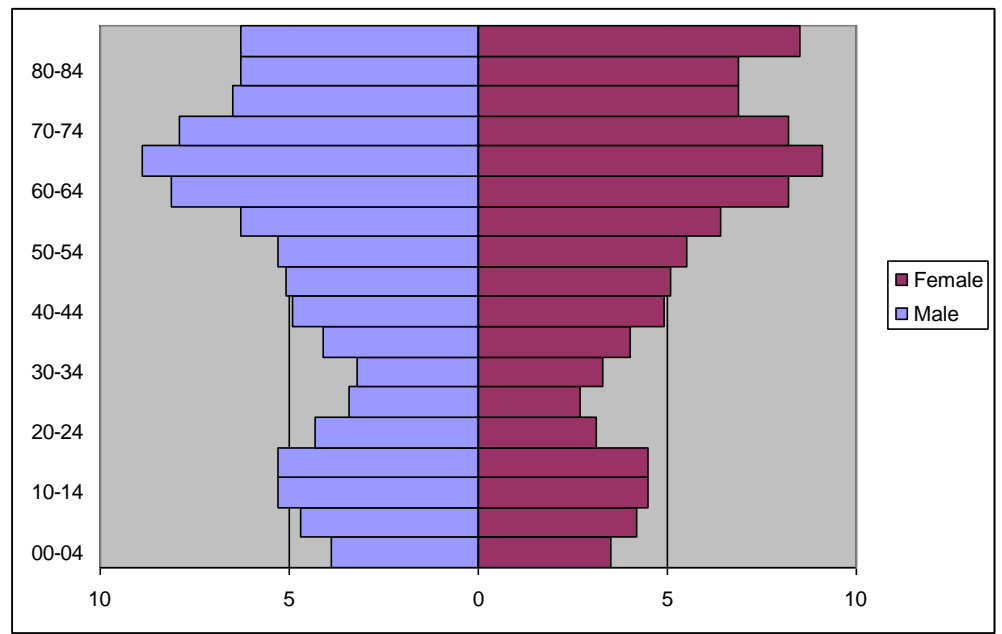

'Source: East Sussex in Figures Rother Census (2001) (www.statistics.gov.uk)'

16b. Population profile for Rother District, 2001 (against UK average)

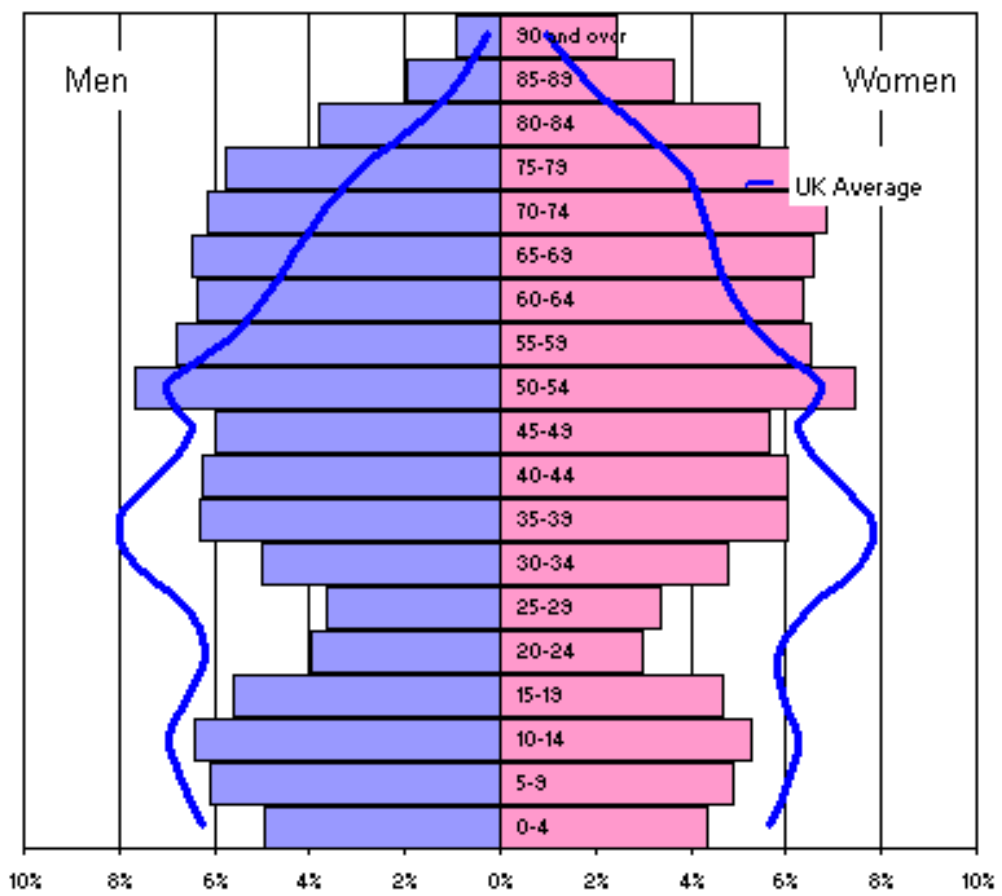

'Source: East Sussex in Figures Rother Census (2001) (www.statistics.gov.uk)' 
16c. Population projection for England 2018 and 2031

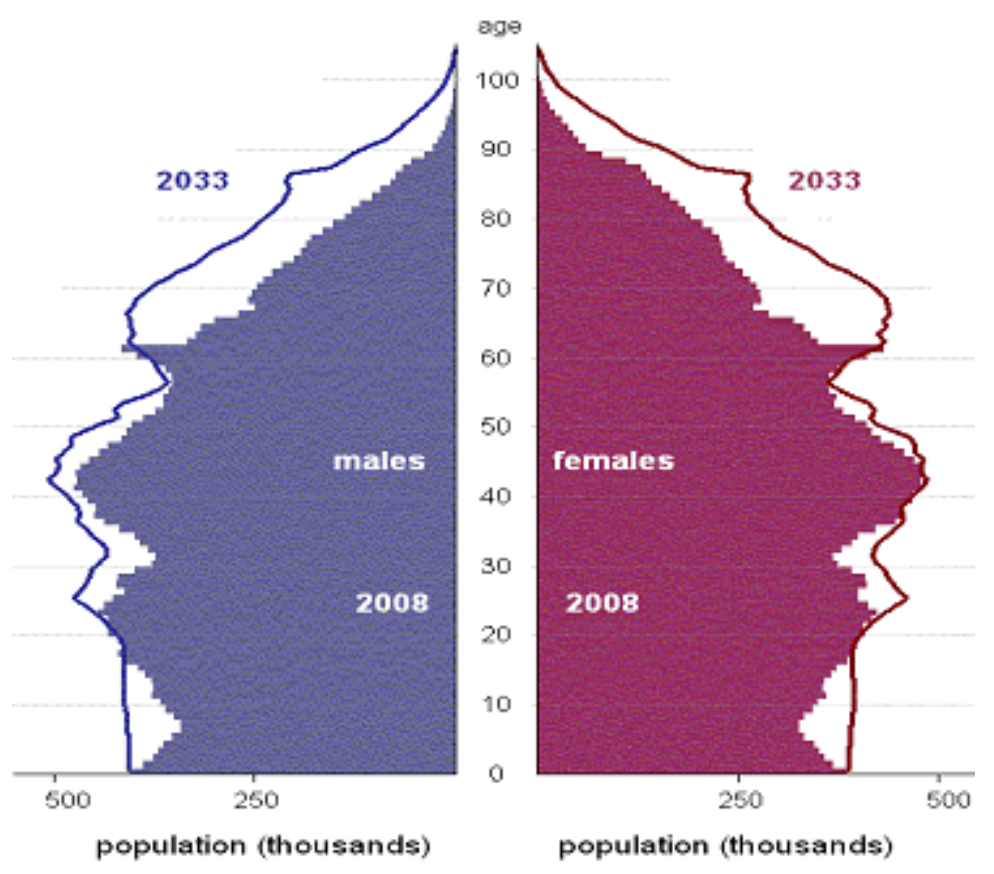

'Source: Office of National Statistics, (2010)' 
Appendix 17

Factors influencing English coastal resort capacity

\begin{tabular}{|c|c|}
\hline $\begin{array}{l}\text { Factors of } \\
\text { influence }\end{array}$ & Evidence of relevance \\
\hline $\begin{array}{l}\text { Failure to } \\
\text { appreciate the } \\
\text { nature, } \\
\text { influence and } \\
\text { consequence } \\
\text { of global forces }\end{array}$ & $\begin{array}{l}\text { Although resort decline has been endemic since the 1970s, } \\
\text { action has been belated (English Tourism Council, 2001). } \\
\text { This is perhaps because there is poor understanding of } \\
\text { resort dynamics (Agarwal, 2002). Furthermore, the } \\
\text { appropriateness of local action has been questioned by } \\
\text { Agarwal (2002), who contends that it focuses on the } \\
\text { symptoms rather than the causes of decline. }\end{array}$ \\
\hline $\begin{array}{l}\text { Degree to } \\
\text { which there is } \\
\text { public sector } \\
\text { commitment }\end{array}$ & $\begin{array}{l}\text { Cooper (1997) argues there has been a major civic } \\
\text { commitment to resort restructuring, however, Agarwal } \\
\text { (1997) revealed a marked variation in policy response in } \\
\text { County structure and Borough and District local plans along } \\
\text { the South coast of England. }\end{array}$ \\
\hline $\begin{array}{l}\text { The existence } \\
\text { of shared } \\
\text { economic } \\
\text { interests and } \\
\text { trust }\end{array}$ & $\begin{array}{l}\text { This is problematic in English coastal resorts due to the } \\
\text { fragmented structure of the tourism industry, the } \\
\text { involvement of a large number of decision-makers (Cooper, } \\
\text { 1997) and a weak culture of trust amongst firms (Coles and } \\
\text { Shaw, 2006) }\end{array}$ \\
\hline $\begin{array}{l}\text { Strong } \\
\text { institutional } \\
\text { presence }\end{array}$ & $\begin{array}{l}\text { Despite the existence of numerous public and private sector } \\
\text { organisations and agencies, English coastal resorts tend to } \\
\text { be characterised by a weak institutional presence. This is } \\
\text { because seaside resorts have had a low profile amongst } \\
\text { national, regional and local government departments } \\
\text { (English Tourist Council, 2001), and because of local } \\
\text { government reorganisation in } 1974 \text { and 1996, which } \\
\text { amalgamated many independent resorts with surrounding } \\
\text { hinterlands, thereby transferring political interests away } \\
\text { from resorts (Cooper, 1997). }\end{array}$ \\
\hline $\begin{array}{l}\text { Levels of } \\
\text { contract, co- } \\
\text { operation and } \\
\text { information } \\
\text { exchange }\end{array}$ & $\begin{array}{l}\text { There is a tradition of poor co-operation, particularly } \\
\text { between the public and private sectors in English coastal } \\
\text { resorts. According to Cooper (1997) the private sector has } \\
\text { traditionally held a short-term tactical view and has been } \\
\text { poorly disposed to attempts to co-ordinate the industry }\end{array}$ \\
\hline $\begin{array}{l}\text { Existence of } \\
\text { collective } \\
\text { representation } \\
\text { that serves to } \\
\text { minimise costs } \\
\text { and control } \\
\text { rogue } \\
\text { behaviour }\end{array}$ & $\begin{array}{l}\text { This is lacking in English coastal resorts as there is a long } \\
\text { tradition of poor investment and co-operation between the } \\
\text { public and private sectors (English Tourism Council, 2001). } \\
\text { There is also a substantial 'hidden' economy that is } \\
\text { considered part of the make-up of seaside resorts }\end{array}$ \\
\hline
\end{tabular}

'Source: Agarwal and Shaw (2007), modified from Memorandum by the Office of the Deputy Prime Minister (2006)' 


\section{Appendix 18}

\section{Median hourly earnings in England's principal seaside towns, 2007 (ranked by male earnings)}

\begin{tabular}{|c|c|c|}
\hline & Male (£) & Female $(£)$ \\
\hline Western-super-Mare & 13.45 & 9.17 \\
\hline Greater Brighton & 12.32 & 10.33 \\
\hline Bridlington & 12.05 & 8.09 \\
\hline Southend-on-Sea & 12.03 & 9.29 \\
\hline Deal & 11.86 & 8.62 \\
\hline Morecambe/Heysham & 11.76 & 8.13 \\
\hline Southport & 11.76 & 8.72 \\
\hline Whitstable/Herne Bay & 11.67 & 8.62 \\
\hline Folkestone/Hythe & 11.51 & 8.23 \\
\hline Greater Worthing & 11.29 & 8.73 \\
\hline Greater Bournemouth & 10.96 & 8.28 \\
\hline Eastbourne & 10.93 & 8.94 \\
\hline Burnham-on-Sea & 10.71 & 7.56 \\
\hline Greater Blackpool & 10.66 & 7.96 \\
\hline Clacton & 10.65 & 8.55 \\
\hline Hastings/Bexhill & 10.60 & 8.04 \\
\hline Isle of Wight & 10.47 & 7.38 \\
\hline Thanet & 10.35 & 8.01 \\
\hline Falmouth & 10.35 & 8.35 \\
\hline Whitley Bay & 10.31 & 7.85 \\
\hline Bognor Regis & 10.24 & 8.56 \\
\hline Exmouth & 10.03 & 8.52 \\
\hline Sidmouth & 10.03 & 8.52 \\
\hline Dawlish/Teignmouth & 9.68 & 8.03 \\
\hline Scarborough & 9.56 & 7.15 \\
\hline Whitby & 9.56 & 7.15 \\
\hline Great Yarmouth & 9.55 & 7.23 \\
\hline Lowestoft & 9.47 & 7.43 \\
\hline Ilfracombe & 9.21 & 7.45 \\
\hline Weymouth & 9.05 & 8.00 \\
\hline Torbay & 9.03 & 7.28 \\
\hline Skegness & 8.95 & 7.61 \\
\hline Newquay & 8.63 & 7.74 \\
\hline Penzance & 8.23 & 6.94 \\
\hline St Ives & 8.23 & 6.94 \\
\hline Minehead & Not available & Not available \\
\hline Swanage & Not available & Not available \\
\hline Seaside towns & 10.89 & 8.39 \\
\hline South East & 13.24 & 9.80 \\
\hline England & 11.80 & 9.04 \\
\hline
\end{tabular}

'Source: Annual Survey of Hours and Earnings taken from Beatty et al., (2008).

Figures refer to Districts covering seaside towns' 


\section{Appendix 19}

Employment in England's principal seaside towns, 2006

\begin{tabular}{|c|c|c|c|c|c|}
\hline & & $\begin{array}{c}\text { Recorded En } \\
\text { jobs }\end{array}$ & oloyee & $\begin{array}{r}\text { Estimate } \\
\text { employ }\end{array}$ & $\begin{array}{l}\text { total } \\
\text { ent }\end{array}$ \\
\hline & $\begin{array}{l}\text { Employment } \\
\text { rate }\end{array}$ & Actual & $\begin{array}{l}\% \text { as } \\
\text { total of } \\
\text { pop }\end{array}$ & Actual & $\begin{array}{l}\% \text { as } \\
\text { total of } \\
\text { pop }\end{array}$ \\
\hline Whitley Bay & 78 & 10,100 & 26 & 10,900 & 28 \\
\hline Gr'tr Worthing & 78 & 72,300 & 38 & 81,500 & 43 \\
\hline Sidmouth & 78 & 5,100 & 37 & 6,400 & 47 \\
\hline Exmouth & 76 & 9,000 & 26 & 10,100 & 30 \\
\hline Western-super-Mare & 75 & 28,600 & 38 & 31,500 & 41 \\
\hline Newquay & 75 & 9,300 & 40 & 11,100 & 47 \\
\hline Whitstable/Herne Bay & 74 & 16,100 & 23 & 18,400 & 26 \\
\hline Ilfracombe & 74 & 3,400 & 30 & 4,200 & 37 \\
\hline Dawlish/Teignmouth & 74 & 7,100 & 23 & 8,500 & 28 \\
\hline Southend-on-Sea & 74 & 61,200 & 38 & 68,500 & 43 \\
\hline Minehead & 74 & 5,300 & 44 & 6,200 & 51 \\
\hline Gr'tr Bournemouth & 74 & 155,500 & 46 & 175,400 & 52 \\
\hline St. Ives & 73 & 4,600 & 41 & 6,000 & 54 \\
\hline Isle of Wight & 73 & 50,100 & 36 & 58,300 & 42 \\
\hline Southport & 73 & 33,900 & 38 & 38,300 & 42 \\
\hline Hastings/Bexhill & 73 & 40,800 & 32 & 47,100 & 37 \\
\hline Scarborough & 73 & 22,900 & 42 & 26,000 & 47 \\
\hline Burnham-on-Sea & 73 & 6,100 & 32 & 6,900 & 36 \\
\hline Gr'tr Brighton & 72 & 127,500 & 45 & 146,600 & 51 \\
\hline Weymouth & 72 & 15,300 & 29 & 17,200 & 33 \\
\hline Eastbourne & 72 & 38,100 & 40 & 43,200 & 45 \\
\hline Torbay & 72 & 48,600 & 37 & 57,200 & 43 \\
\hline Swanage & 71 & 2,900 & 29 & 3,600 & 36 \\
\hline Bognor Regis & 71 & 12,800 & 30 & 14,600 & 35 \\
\hline Whitby & 71 & 5,300 & 39 & 6,200 & 45 \\
\hline Morecambe/Heysham & 71 & 15,000 & 30 & 16,400 & 32 \\
\hline Gr'tr Blackpool & 70 & 104,800 & 40 & 120,000 & 45 \\
\hline Skegness & 70 & 8,000 & 39 & 9,400 & 46 \\
\hline Deal & 69 & 5,200 & 18 & 5,800 & 20 \\
\hline Penzance & 69 & 8,100 & 38 & 10,100 & 47 \\
\hline Falmouth & 68 & 7,200 & 34 & 8,400 & 40 \\
\hline Folkestone/Hythe & 68 & 22,600 & 38 & 25,000 & 42 \\
\hline Lowestoft & 68 & 21,800 & 34 & 23,600 & 37 \\
\hline Thanet & 67 & 35,300 & 29 & 39,700 & 33 \\
\hline Bridlington & 65 & 9,700 & 25 & 11,400 & 29 \\
\hline Great Yarmouth & 65 & 28,300 & 49 & 31,100 & 53 \\
\hline Clacton & 62 & 15,000 & 26 & 17,100 & 30 \\
\hline Seaside towns & 72 & $1,072,900$ & 37 & $1,221,900$ & 43 \\
\hline South East & 79 & $3,668,700$ & 45 & $4,108,300$ & 50 \\
\hline England & 74 & $22,766,600$ & 45 & $25,192,500$ & 50 \\
\hline
\end{tabular}

'Source: Annual Business Inquiry, Census of Population adapted from Beatty et al., (2008)' Note: The table presents two figures. The first is the number of employee jobs recorded by the government's Annual Business Enquiry; the second is an estimate of the total number of people in work (adding in self-employed and deducting double-jobbing). 


\section{Appendix 20}

Economic inactivity among working age residents of seaside towns, 19812001

\begin{tabular}{|c|c|c|c|}
\hline & \multicolumn{3}{|c|}{$\%$ of working age population } \\
\hline & 1981 & 1991 & 2001 \\
\hline \multicolumn{4}{|l|}{ Men } \\
\hline Permanently sick & 3.7 & 5.5 & 9.0 \\
\hline Students & 5.7 & 4.5 & \multirow{3}{*}{7.5} \\
\hline Retired & 0.9 & 3.2 & \\
\hline Other inactive & 0.8 & 0.9 & \\
\hline All inactive of working age & 11.1 & 14.1 & 16.5 \\
\hline \multicolumn{4}{|l|}{ Women } \\
\hline Permanently sick & 3.0 & 3.4 & 5.9 \\
\hline Students & 5.6 & 4.7 & \multirow{3}{*}{ \} 21.0} \\
\hline Retired & \multirow[t]{2}{*}{31.9} & 1.3 & \\
\hline Other inactive & & 22.7 & \\
\hline All inactive of working age & 40.5 & 32.2 & 26.9 \\
\hline
\end{tabular}

'Source: Census of Population, Labour Force Survey, Department for Work and Pensions taken from Beatty, C. and Fothergill, S., (2004)' 


\section{Appendix 21}

\section{Working age and Pension credit claimant rates in England's principal seaside towns, May 2007 (working age ranked by total)}

\begin{tabular}{|c|c|c|c|c|c|}
\hline & \multicolumn{4}{|c|}{$\%$ of working age population } & \multirow{2}{*}{ 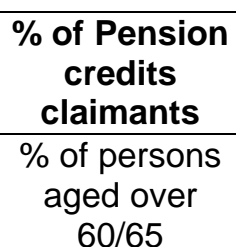 } \\
\hline & JSA & $\begin{array}{l}\text { IS as } \\
\text { lone } \\
\text { parent }\end{array}$ & $\mathrm{IB} / \mathrm{SDA}$ & Total & \\
\hline Skegness & 3.4 & 2.5 & 14.6 & 20.5 & 34.0 \\
\hline Great Yarmouth & 5.2 & 3.2 & 11.6 & 20.0 & 33.8 \\
\hline Clacton & 3.7 & 3.4 & 12.2 & 19.3 & 25.7 \\
\hline Penzance & 2.8 & 2.6 & 13.2 & 18.6 & 29.0 \\
\hline Bridlington & 3.5 & 2.3 & 11.7 & 17.4 & 28.8 \\
\hline Ilfracombe & 2.3 & 2.8 & 11.8 & 16.9 & 33.3 \\
\hline Hastings/Bexhill & 3.0 & 2.9 & 10.3 & 16.2 & 23.4 \\
\hline Thanet & 3.3 & 3.0 & 9.8 & 16.1 & 28.6 \\
\hline Lowestoft & 4.3 & 2.7 & 9.0 & 15.9 & 26.0 \\
\hline Gr'tr Blackpool & 2.4 & 2.2 & 11.2 & 15.8 & 26.3 \\
\hline Morecambe/Heysham & 3.0 & 2.2 & 10.6 & 15.8 & 28.1 \\
\hline Western-super-Mare & 1.8 & 2.4 & 11.3 & 15.4 & 26.1 \\
\hline Scarborough & 3.2 & 2.7 & 9.4 & 15.4 & 28.3 \\
\hline Torbay & 2.4 & 2.3 & 10.2 & 14.9 & 26.2 \\
\hline Folkestone/Hythe & 3.6 & 2.7 & 8.3 & 14.6 & 25.8 \\
\hline Southend-on-Sea & 2.9 & 2.7 & 7.7 & 13.4 & 24.8 \\
\hline Weymouth & 1.6 & 1.7 & 9.4 & 12.7 & 21.1 \\
\hline Eastbourne & 2.5 & 2.2 & 8.0 & 12.7 & 20.6 \\
\hline Burnham-on-Sea & 1.6 & 1.7 & 9.3 & 12.6 & 22.5 \\
\hline Minehead & 1.5 & 1.8 & 9.2 & 12.6 & 18.4 \\
\hline Whitby & 2.3 & 1.8 & 8.4 & 12.5 & 19.7 \\
\hline Gr'tr Brighton & 2.5 & 2.1 & 7.5 & 12.1 & 25.8 \\
\hline Isle of Wight & 2.1 & 2.1 & 7.9 & 12.1 & 22.1 \\
\hline Dawlish/Teighmouth & 1.2 & 1.6 & 9.0 & 11.9 & 21.0 \\
\hline Southport & 1.9 & 1.5 & 8.3 & 11.8 & 23.4 \\
\hline Newquay & 2.0 & 1.6 & 8.0 & 11.7 & 25.2 \\
\hline Deal & 1.9 & 1.7 & 7.8 & 11.5 & 22.7 \\
\hline Falmouth & 1.8 & 1.9 & 7.7 & 11.5 & 26.1 \\
\hline St Ives & 1.6 & 1.3 & 8.5 & 11.4 & 23.2 \\
\hline Bognor Regis & 2.0 & 1.8 & 7.2 & 10.9 & 19.7 \\
\hline Gr'tr Bournemouth & 1.4 & 1.9 & 7.4 & 10.7 & 21.9 \\
\hline Gr'tr Worthing & 1.3 & 1.7 & 6.8 & 9.9 & 18.1 \\
\hline Whitstable/Herne Bay & 1.7 & 1.6 & 6.5 & 9.7 & 21.4 \\
\hline Exmouth & 1.1 & 1.2 & 7.0 & 9.3 & 20.0 \\
\hline Whitley Bay & 1.7 & 0.7 & 5.6 & 8.0 & 19.7 \\
\hline Swanage & 0.7 & 1.3 & 5.4 & 7.3 & 17.1 \\
\hline Sidmouth & 0.7 & 0.8 & 4.9 & 6.4 & 10.8 \\
\hline Seaside towns & 2.4 & 2.2 & 8.8 & 13.3 & 24.0 \\
\hline South East & 1.4 & 1.5 & 4.8 & 7.7 & 17.9 \\
\hline England & 2.3 & 2.1 & 6.9 & 11.2 & 24.2 \\
\hline
\end{tabular}

'Source: DWP, ONS taken from Beatty et al., (2008)'

Note: JSA (Jobseekers Allowance), IS (Income Support) IB/SDA (Incapacity Benefit/Severe Disability Allowance). These three benefits are mutually exclusive so benefit claimants cannot receive more than one at the same time

Note: Although take-up of pension credits is less than comprehensive, this is a guide to the distribution of pensioners living on low incomes 
Across Britain as a whole, incapacity claimants are a predominantly older group: the nearer the state pension age, the greater the likelihood of claiming incapacity benefit. This is understandable because advancing age is often associated with declining health and rising disability (DWP, 2009a). Incapacity benefits are paid to men and women of working age who are out-of work but deemed too ill or disabled to be required to look for work. This differentiates them sharply from the claimant unemployed in that those in receipt of jobseeker's allowance are required to look for work as a condition of benefit receipt. These two groups of benefit claimants are mutually exclusive: it is not possible to claim incapacity benefit and jobseeker's allowance at the same time (DWP, 2010a). Importantly, there is also in most circumstances a financial incentive to claim incapacity benefit rather than jobseeker's allowance, as the latter is means tested whilst the former is mostly not means tested (Beatty and Fothergill, 2007b).

These differences in benefit rules, the large increase in the number of incapacity benefit claimants since the late 1970s, and the concentration of incapacity benefit claimants in many of the weakest labour markets across England have all fuelled the argument that incapacity benefits hide substantial unemployment. A paper that studied 'Changes in the profile of men claiming Incapacity Benefits - a case study in Barrow-in-Furness', (Beatty and Fothergill, 2007b) identified that the findings were consistent with the view that as redundancies from older industries recede into the past, a new group of less healthy, less skilled and less motivated men is becoming marginalised. One irony about this is that seaside towns began life as healthy therapeutic venues for the upper and middle classes, but now contain above-average numbers of unhealthy people claiming incapacity and disability benefits (Walton, and Browne, 2010) 


\section{Appendix 22}

\section{Bexhill Detailed Locality Data for some of the LSOAs, January 2012}

\section{Contents}

\begin{tabular}{|l|l|l|}
\hline LSOA ref & Ward & Locality \\
\hline $007 \mathrm{E}$ & Sidley & Glovers Lane Area \\
\hline $007 \mathrm{D}$ & Sidley & Buxton Drive Area \\
\hline $007 \mathrm{~F}$ & Sidley & West Sidley \\
\hline $009 \mathrm{G}$ & Sidley & Outer fringe \\
\hline $011 \mathrm{C}$ & Central & Amherst Road Area \\
\hline $011 \mathrm{~B}$ & Central & Town Centre \\
\hline $011 \mathrm{~A}$ & Central & Reginald Road Area \\
\hline $009 \mathrm{C}$ & St Marks & Peartree Lane area \\
\hline $009 \mathrm{D}$ & St Marks & Barnhorn Road area \\
\hline $009 \mathrm{E}$ & St Marks & Cooden Sea Road and fringes \\
\hline $008 \mathrm{C}$ & St Michaels & North De La Warr Road \\
\hline $008 \mathrm{D}$ & St Michaels & East Pebsham \\
\hline $008 \mathrm{E}$ & St Michaels & West Pebsham \\
\hline
\end{tabular}

'Source: East Sussex in Figures: www.eastsussexinfigures.org.uk Accessed 10 February (2012) ONS: www.neighbourhood.statistics.gov.uk Accessed 10 February (2012)'

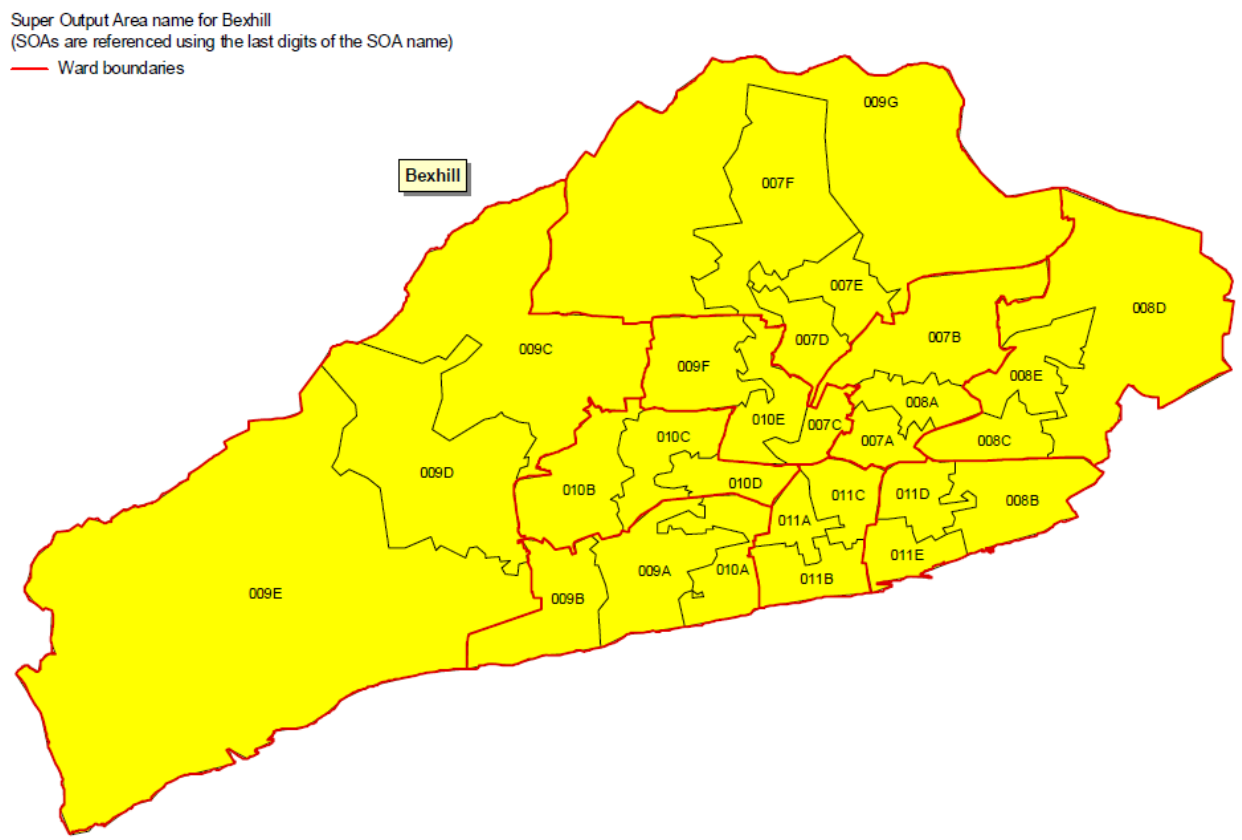




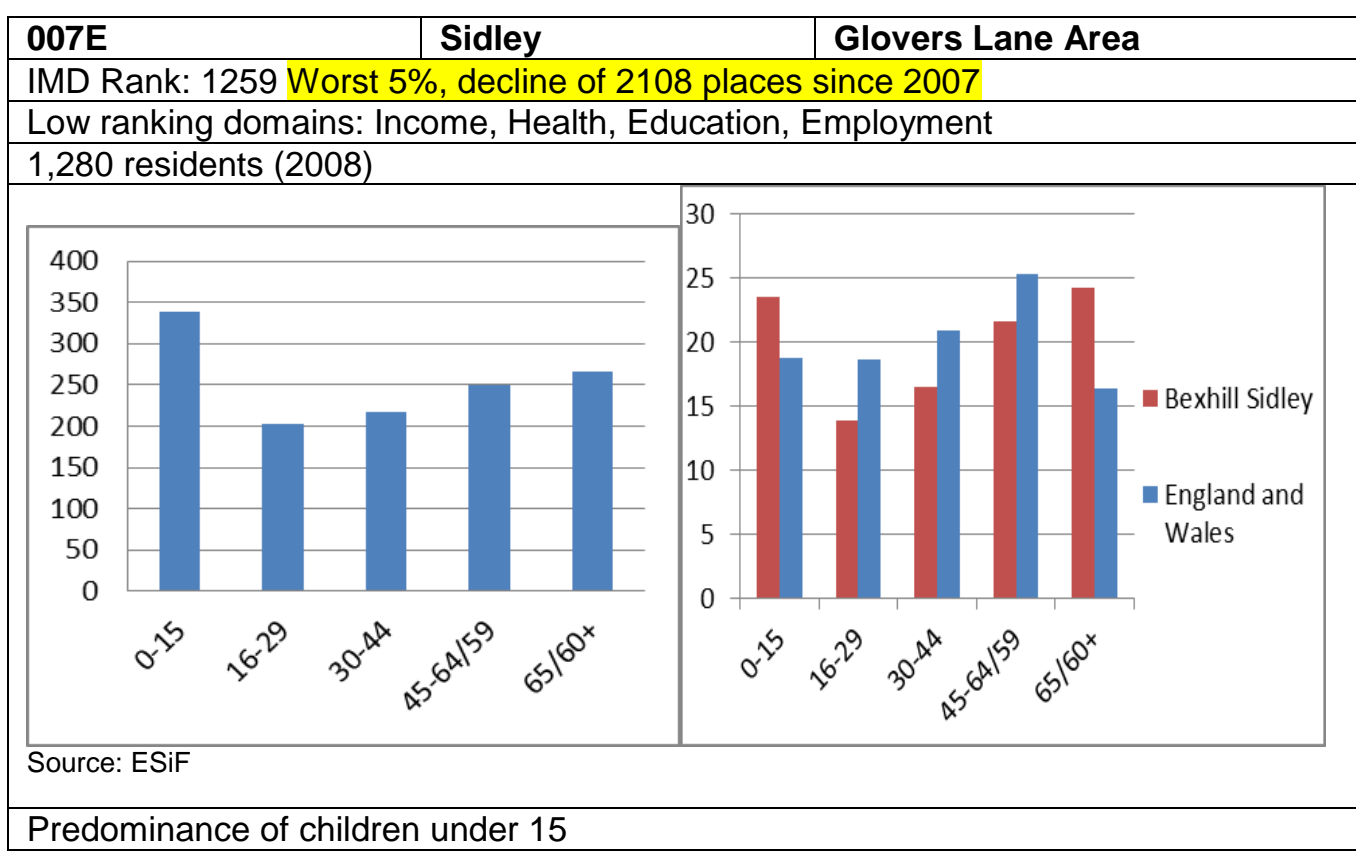

\begin{tabular}{|l|l|l|l|}
\hline & & Rother & England \\
\hline Benefits August 2010 & $\%$ & $\%$ & $\%$ \\
\hline Any working age benefit & 41 & 15 & 15 \\
\hline JSA & 8 & 3 & 4 \\
\hline Incapacity & 19 & 7 & 7 \\
\hline Lone parents & & & \\
\hline Youth unemployment & 38 & 30 & 27 \\
\hline Fuel poverty & 11 & & \\
\hline Pensions Credit, count & 140 & 4840 & 2287930 \\
\hline Occupations & & & \\
\hline Elementary & 20 & 11 & 12 \\
\hline Personal services & 14 & 9 & 7 \\
\hline Sales & 13 & 7 & 8 \\
\hline Skilled trades & 12 & 14 & 11 \\
\hline Health & & & \\
\hline Good & 57 & 65 & 68 \\
\hline Fairly good & 28 & 25 & 22 \\
\hline Not good & 14 & 9 & 9 \\
\hline Limiting illness, working age & 23 & 14 & 13 \\
\hline Carers, high level unpaid caring & 30 & 19 & 20 \\
\hline Education & & & \\
\hline Adults low/no qualifications & 57 & & \\
\hline $\begin{array}{l}\text { 5+ GCSEs A*-C, including English } \\
\text { and Maths }\end{array}$ & 40 & 59 & 55 \\
\hline Crime & & & \\
\hline Violent crime: rank of 18 wards & 2 & & \\
\hline Criminal Damage: rank of 18 wards & 2 & & \\
\hline Burglary Dwelling: rank of 18 wards & 5 & & \\
\hline $\begin{array}{l}\text { Theft from Motor vehicle: rank of } 18 \\
\text { wards }\end{array}$ & 3 & & \\
\hline Shoplifting: rank of 18 wards & 4 & & \\
\hline & & & \\
\hline
\end{tabular}




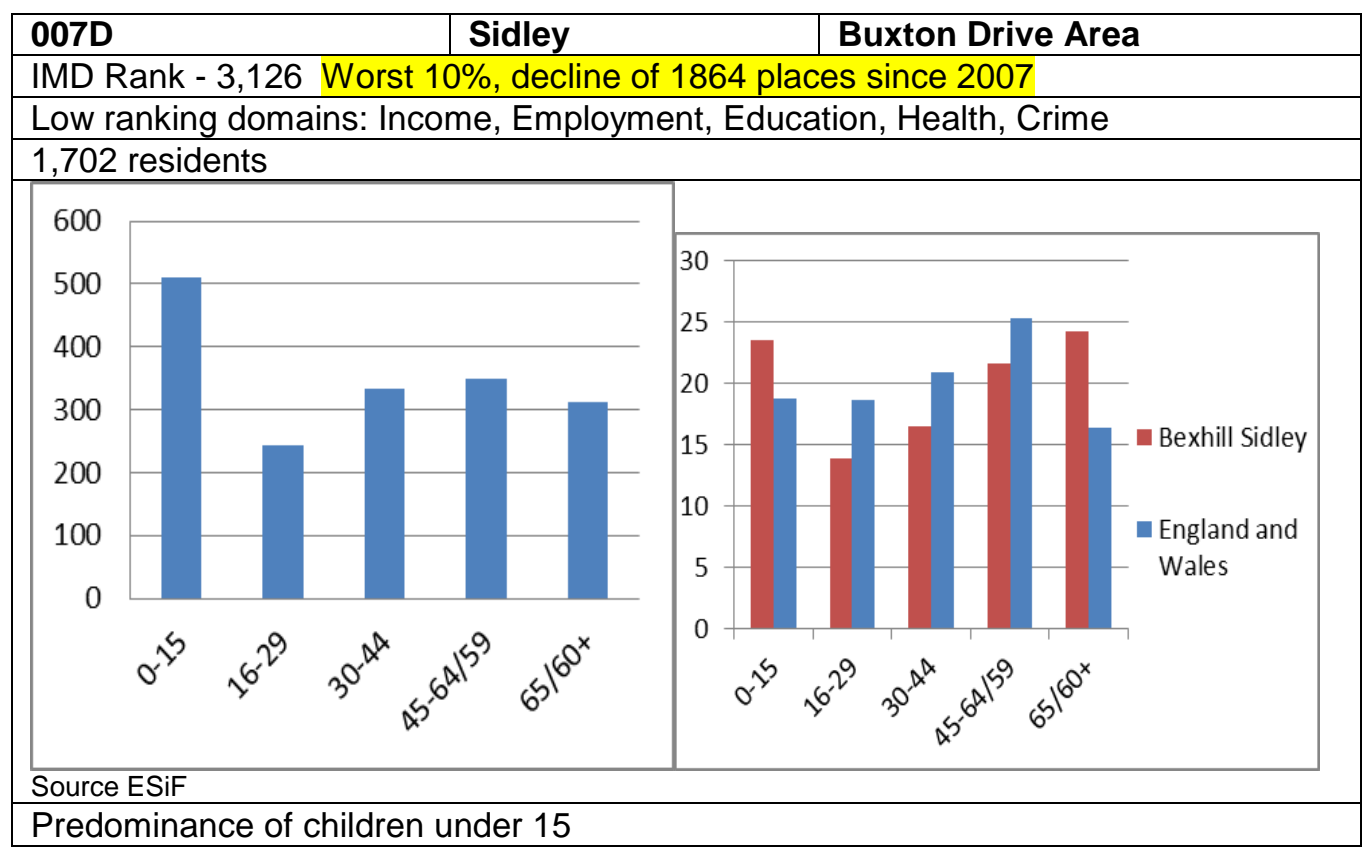

\begin{tabular}{|l|l|l|l|}
\hline & & Rother & England \\
\hline Benefit claimants August 2010 & $\%$ & $\%$ & $\%$ \\
\hline Any working age benefit & 33 & 15 & 15 \\
\hline JSA & 7 & 3 & 4 \\
\hline Incapacity & 14 & 7 & 7 \\
\hline Lone parents & & & \\
\hline JSA youth 18-24 age & 38 & 30 & 28 \\
\hline Fuel poverty (households) & 11 & & \\
\hline Pension Credit, count & 110 & 4840 & 2287930 \\
\hline Occupations & & & \\
\hline Elementary & 19 & 11 & 12 \\
\hline Skilled trades & 17 & 14 & 12 \\
\hline Personal service & 13 & 9 & 7 \\
\hline Process, plant, machine & 10 & 5 & 8 \\
\hline Administrative & 10 & 13 & 13 \\
\hline Health & & & \\
\hline Good & 62 & 65 & 68 \\
\hline Fairly good & 27 & 25 & 22 \\
\hline Not good & 11 & 9 & 9 \\
\hline Working age limiting illness & 19 & 14 & 13 \\
\hline High level unpaid caring & 26 & 19 & 20 \\
\hline Education & & & \\
\hline Adults low/no qualifications & 58 & & \\
\hline $\begin{array}{l}\text { 5+ GCSEs A } \\
\text { and Maths } \text { including English }\end{array}$ & 36 & 59 & 55 \\
\hline Crime & & & \\
\hline Violent crime: rank of 18 wards & 2 & & \\
\hline Criminal Damage: rank of 18 wards & 2 & & \\
\hline Burglary Dwelling: rank of 18 wards & 5 & & \\
\hline $\begin{array}{l}\text { Theft from Motor vehicle: rank of } 18 \\
\text { wards }\end{array}$ & 3 & & \\
\hline Shoplifting: rank of 18 wards & 4 & & \\
\hline & & & \\
\hline
\end{tabular}




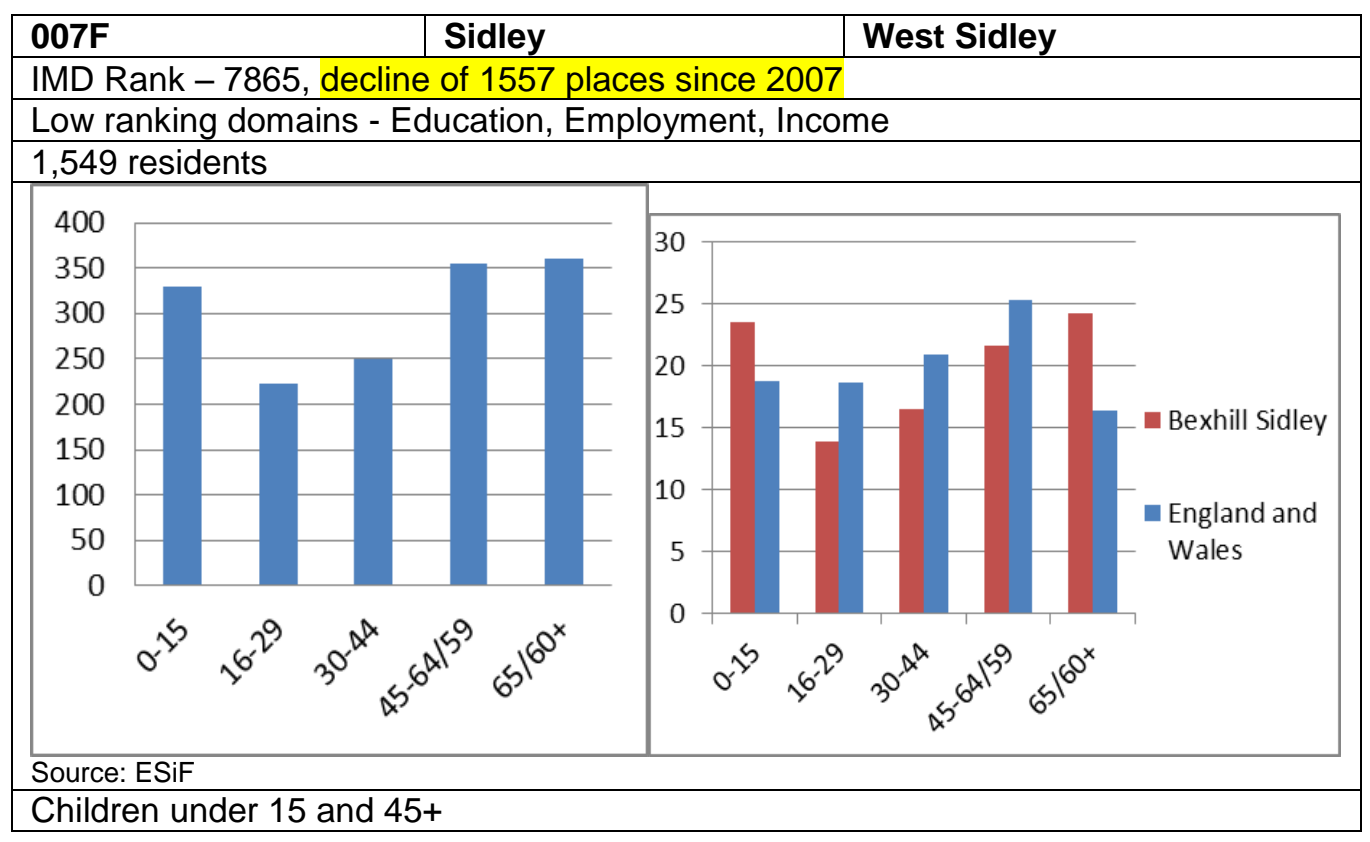

\begin{tabular}{|l|l|l|l|}
\hline & & Rother & England \\
\hline Benefits claimants August 2010 & $\%$ & $\%$ & $\%$ \\
\hline Any key working age benefit & 25 & 15 & 15 \\
\hline JSA & 6 & 3 & 4 \\
\hline Incapacity & 10 & 7 & 7 \\
\hline Youth JSA & & & \\
\hline Fuel poverty (households) & 13 & & \\
\hline Pension Credit, count & 130 & 4848 & 2287930 \\
\hline Occupations & & & \\
\hline Skilled trades & 16 & 14 & 11 \\
\hline Elementary & 14 & 11 & 12 \\
\hline Managers, senior officials & 13 & 17 & 15 \\
\hline Health & & & \\
\hline Good & 63 & 65 & 68 \\
\hline Fairly good & 27 & 25 & 22 \\
\hline Not good & 9 & 9 & 9 \\
\hline Working age limiting illness & 15 & 14 & 13 \\
\hline High level unpaid caring & 27 & 19 & 20 \\
\hline Education & & & \\
\hline Adults low/no qualifications & 54 & & \\
\hline $\begin{array}{l}\text { 5+ GCSEs A*-C, including English } \\
\text { and Maths }\end{array}$ & $\mathrm{n} / \mathrm{a}$ & 59 & 55 \\
\hline Crime & & & \\
\hline Violent crime: rank of 18 wards & 2 & & \\
\hline Criminal Damage: rank of 18 wards & 2 & & \\
\hline Burglary Dwelling: rank of 18 wards & 5 & & \\
\hline $\begin{array}{l}\text { Theft from Motor vehicle: rank of } 18 \\
\text { wards }\end{array}$ & 3 & & \\
\hline Shoplifting: rank of 18 wards & 4 & & \\
\hline & & & \\
\hline
\end{tabular}




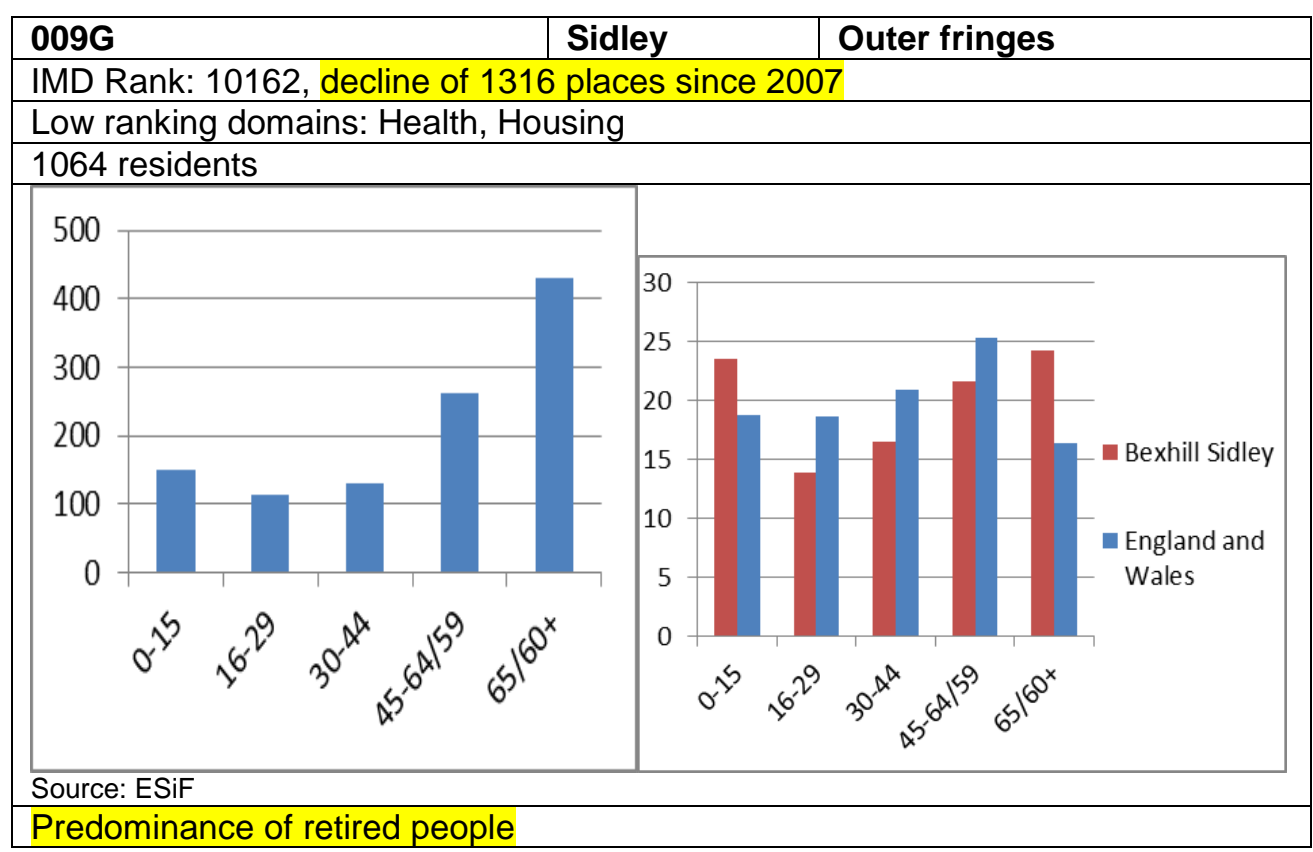

\begin{tabular}{|l|l|l|l|}
\hline & & Rother & England \\
\hline Benefits claimants August 2010 & $\%$ & $\%$ & $\%$ \\
\hline Any working age benefit & 22 & 15 & 15 \\
\hline JSA & 3 & 3 & 4 \\
\hline Incapacity & 12 & 7 & 7 \\
\hline Lone parents, count & 12 & 1733 & 1311974 \\
\hline Fuel poverty (households) & 18 & & \\
\hline Pension Credit, count & 75 & 4840 & 2287930 \\
\hline Occupations & & & \\
\hline Managers and senior officials & 17 & 17 & 15 \\
\hline Associate professionals & 12 & 14 & 14 \\
\hline Administrative & 17 & 13 & 13 \\
\hline Health & & & \\
\hline Good & 59 & 65 & 68 \\
\hline Fairly good & 30 & 25 & 22 \\
\hline Not good & 12 & 9 & 9 \\
\hline Working age limiting illness & 18 & 14 & 13 \\
\hline High level unpaid caring & 28 & 19 & 20 \\
\hline Education & & & \\
\hline $\begin{array}{l}\text { 5+ GCSEs A*-C, including English } \\
\text { and Maths }\end{array}$ & 43 & 59 & 55 \\
\hline Crime & & & \\
\hline Violent crime: rank of 18 wards & 2 & & \\
\hline Criminal Damage: rank of 18 wards & 2 & & \\
\hline Burglary Dwelling: rank of 18 wards & 5 & & \\
\hline $\begin{array}{l}\text { Theft from Motor vehicle: rank of } \\
\text { 18 wards }\end{array}$ & 3 & & \\
\hline Shoplifting: rank of 18 wards & 4 & & \\
\hline & & & \\
\hline & & & \\
\hline & & & \\
\hline
\end{tabular}




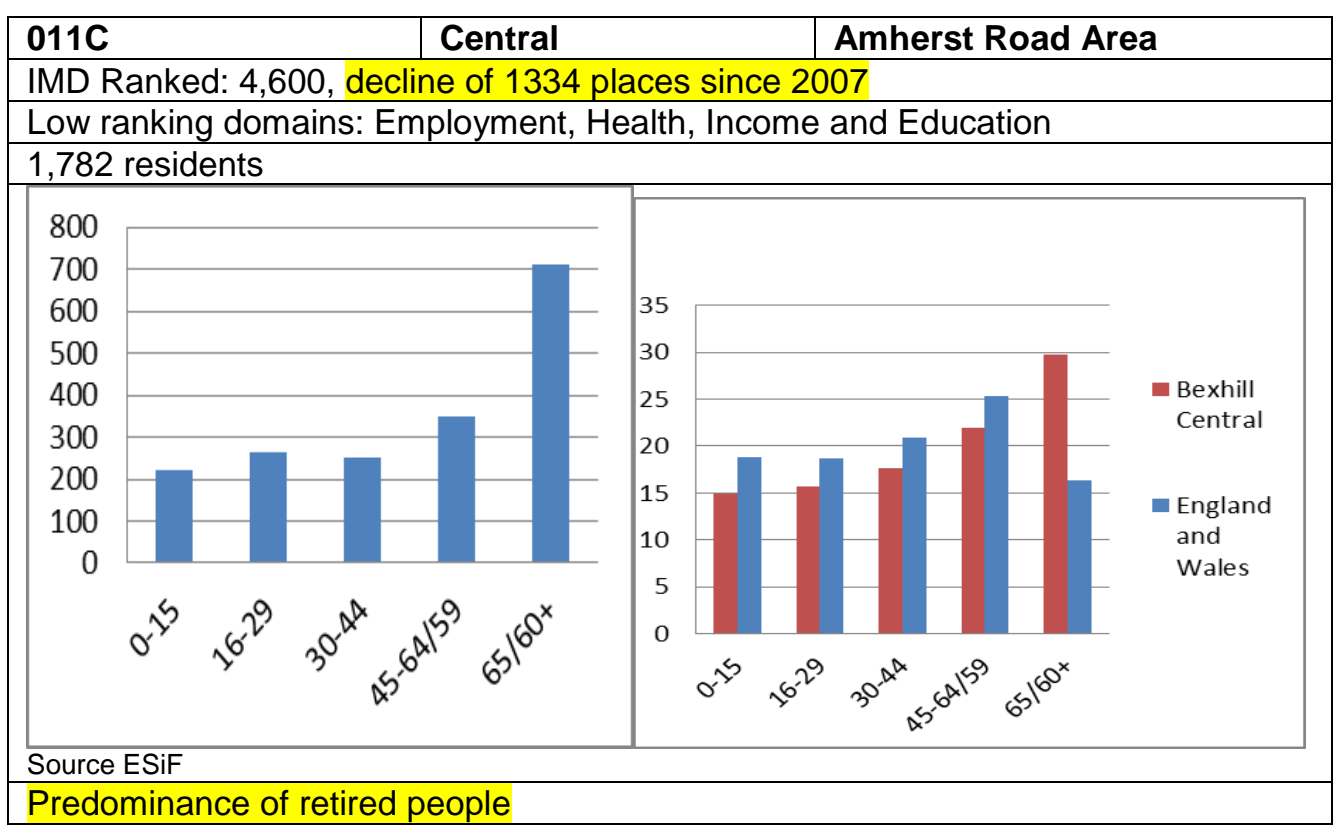

\begin{tabular}{|l|l|l|l|}
\hline & & Rother & England \\
\hline Benefits claimants August 2010 & $\%$ & $\%$ & $\%$ \\
\hline Any working age benefit & 28 & 15 & 15 \\
\hline JSA & 7 & 3 & 4 \\
\hline Incapacity & 15 & 7 & 7 \\
\hline Lone parents & & & \\
\hline JSA youth 18-24 age & & & \\
\hline Fuel poverty (households) & 17 & & \\
\hline Pension credit, count & 55 & 4840 & 2287930 \\
\hline Occupations & & & \\
\hline Managers & 14 & 17 & 15 \\
\hline Administrative & 16 & 13 & 13 \\
\hline Associate professionals & 13 & 13 & 13 \\
\hline Health & & & \\
\hline Good & 53 & 65 & 68 \\
\hline Fairly good & 32 & 25 & 22 \\
\hline Not good & 15 & 9 & 9 \\
\hline Working age limiting illness & 25 & 14 & 13 \\
\hline High level unpaid caring & 23 & 19 & 20 \\
\hline Education & & & \\
\hline $\begin{array}{l}5+\text { GCSEs A*-C, including English } \\
\text { and Maths }\end{array}$ & 33 & 59 & 55 \\
\hline Adults low/no qualifications & 47 & & \\
\hline Crime & 1 & & \\
\hline Violent crime: rank of 18 wards & 1 & & \\
\hline $\begin{array}{l}\text { Criminal damage : rank of } 18 \\
\text { wards }\end{array}$ & 1 & & \\
\hline Burglary: rank of 18 wards & 1 & & \\
\hline Theft from Motor: rank of 18 wards & 4 & & \\
\hline Stolen Motor & 1 & & \\
\hline Robbery: rank of 18 wards & 1 & & \\
\hline $\begin{array}{l}\text { Theft from person: rank of } 18 \\
\text { wards }\end{array}$ & 1 & & \\
\hline Shoplifting: rank of 18 wards & 1 & & \\
\hline & & & \\
\hline & & & \\
\hline & & & \\
\hline
\end{tabular}




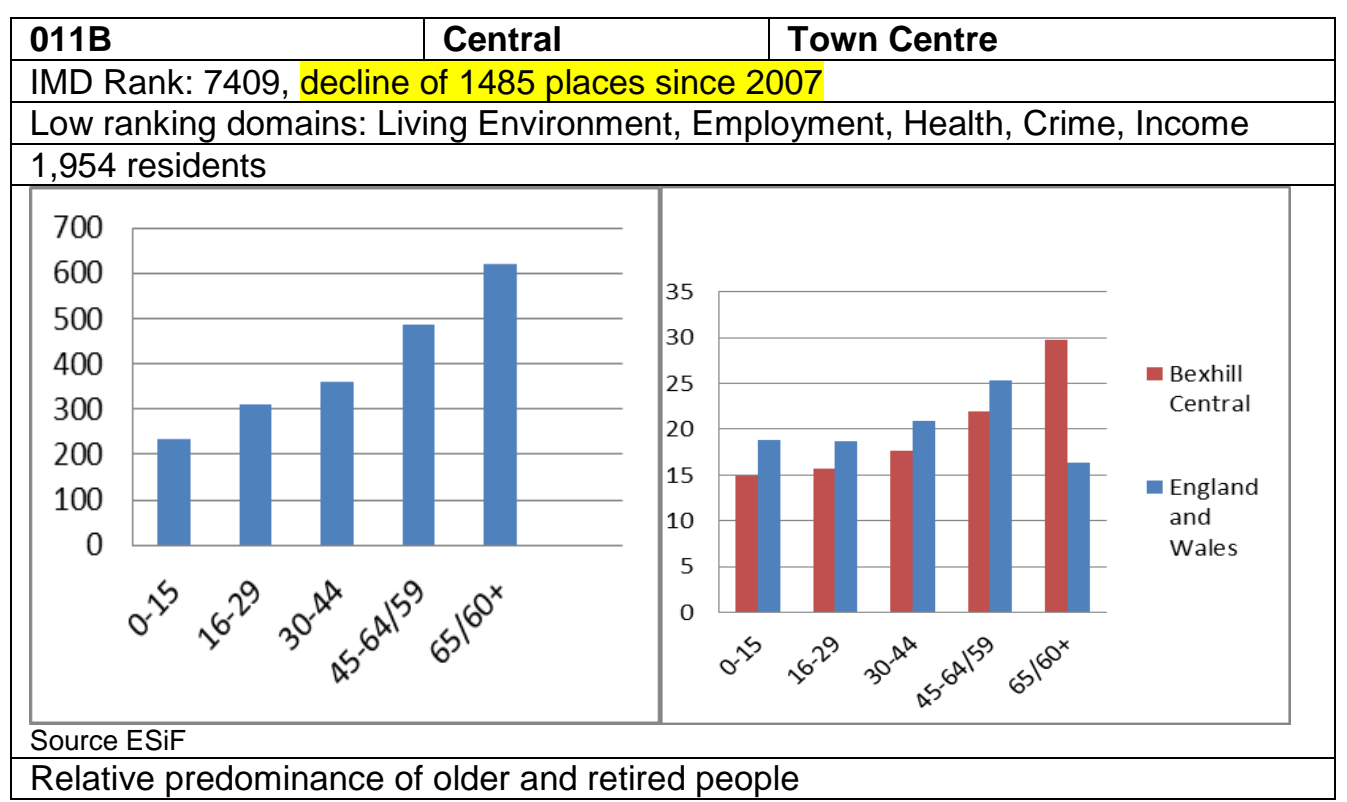

\begin{tabular}{|l|l|l|l|}
\hline & & Rother & England \\
\hline Benefits claimants August 2010 & $\%$ & $\%$ & $\%$ \\
\hline Any key working age benefit & 25 & 15 & 15 \\
\hline JSA & 6 & 3 & 4 \\
\hline Incapacity & 14 & 7 & 7 \\
\hline Fuel Poverty & 21 & & \\
\hline Pension credit count & 160 & 4840 & 2287930 \\
\hline Occupations & & & \\
\hline Managers & 14 & 17 & 15 \\
\hline Professionals & 13 & 10 & 11 \\
\hline Skilled trades & 12 & 14 & 11 \\
\hline Elementary & 13 & 11 & 12 \\
\hline Health & & & \\
\hline Good & 57 & 65 & 68 \\
\hline Fairly good & 30 & 25 & 22 \\
\hline Not good & 13 & 9 & 9 \\
\hline Limiting long-term illness & 19 & 14 & 13 \\
\hline High level unpaid caring & 17 & 19 & 20 \\
\hline Education & & & \\
\hline $\begin{array}{l}5+\text { GCSEs A*-C, including English } \\
\text { and Maths }\end{array}$ & 46 & 59 & 55 \\
\hline Adults low/no qualifications & 45 & & \\
\hline Crime & & & \\
\hline Violent crime: rank of 18 wards & 1 & & \\
\hline Criminal damage : rank of 18 wards & 1 & & \\
\hline Burglary: rank of 18 wards & 1 & & \\
\hline Theft from Motor: rank of 18 wards & 4 & & \\
\hline Stolen Motor & 1 & & \\
\hline Robbery: rank of 18 wards & 1 & & \\
\hline Theft from person: rank of 18 wards & 1 & & \\
\hline Shoplifting: rank of 18 wards & 1 & & \\
\hline & & & \\
\hline
\end{tabular}




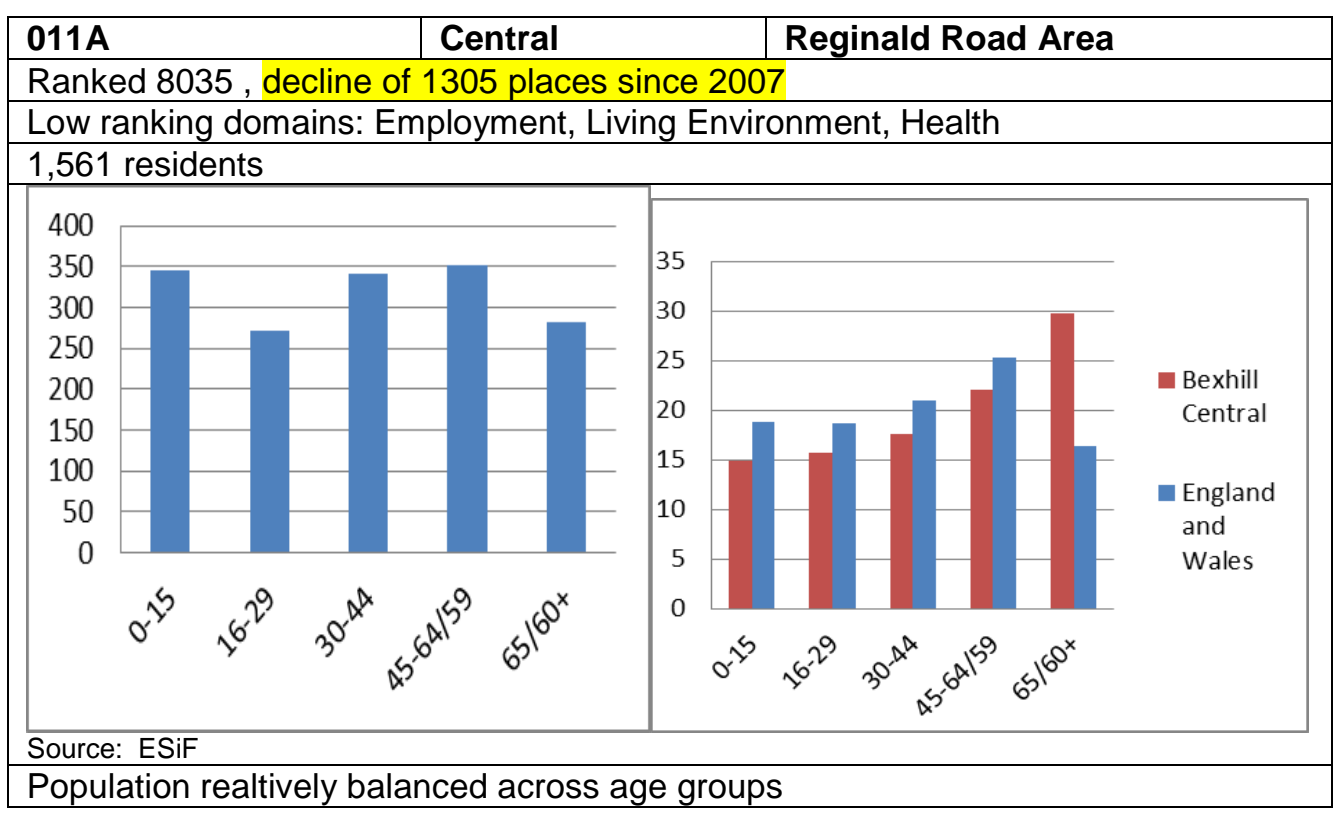

\begin{tabular}{|l|l|l|l|}
\hline & & Rother & England \\
\hline Benefits claimants August 2010 & $\%$ & $\%$ & $\%$ \\
\hline Any working age benefit & 22 & 15 & 15 \\
\hline JSA & 5 & 3 & 4 \\
\hline Incapacity & 9 & 7 & 7 \\
\hline Lone parents, count & 65 & 1733 & 1311974 \\
\hline Youth JSA & & & \\
\hline Fuel Poverty & 19 & & \\
\hline Pension credit, count & 105 & 4840 & 2287930 \\
\hline Occupations & & & \\
\hline Administrative & 14 & 12 & 13 \\
\hline Managers & 15 & 17 & 15 \\
\hline Elementary & 12 & 11 & 12 \\
\hline Health & & & \\
\hline Good & 65 & 65 & 68 \\
\hline Fairly good & 26 & 25 & 22 \\
\hline Not good & 9 & 9 & 9 \\
\hline Working age limiting illness & 15 & 14 & 13 \\
\hline High level unpaid caring & 14 & 19 & 20 \\
\hline Education & & & \\
\hline $\begin{array}{l}\text { 5+ GCSEs A*-C, including English } \\
\text { and Maths }\end{array}$ & 63 & 59 & 55 \\
\hline Adults low/no qualifications & 47 & & \\
\hline Crime & & & \\
\hline As above & & & \\
\hline
\end{tabular}




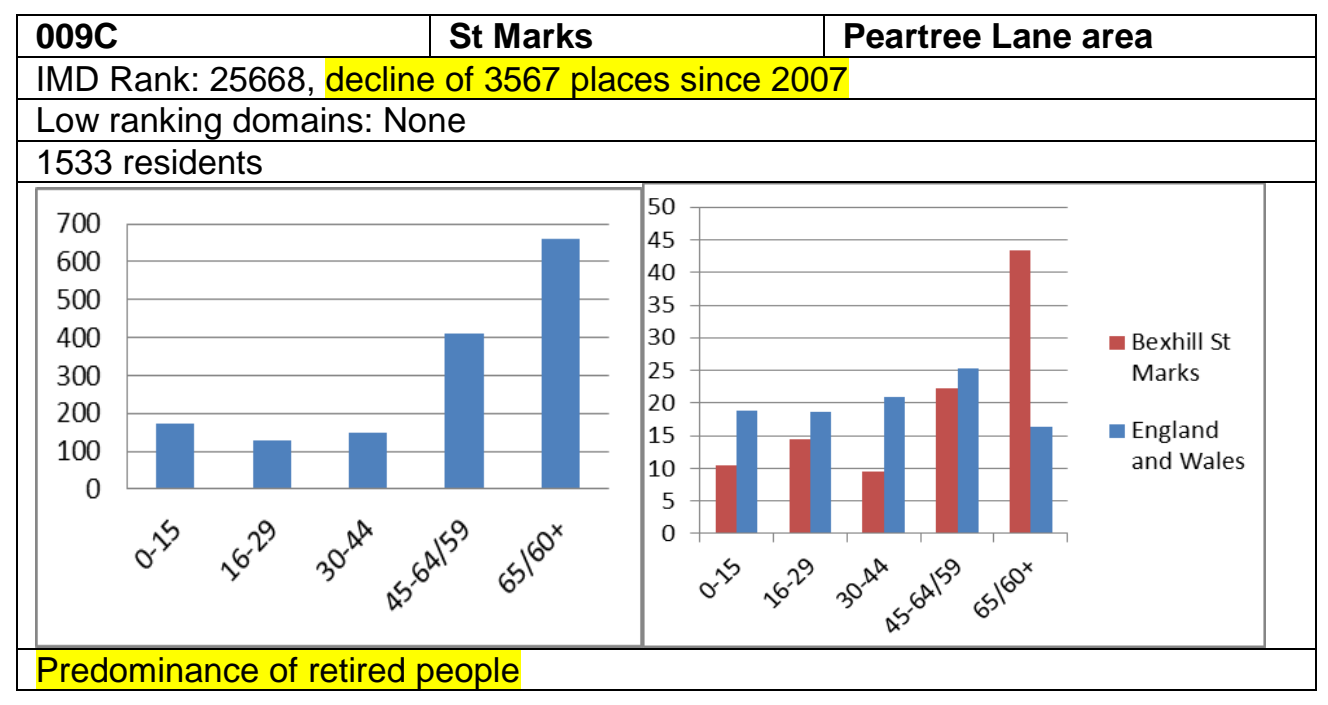

\begin{tabular}{|l|l|l|l|}
\hline & & Rother & England \\
\hline Benefits claimants August 2010 & $\%$ & $\%$ & $\%$ \\
\hline Any key working age benefit & 11 & 15 & 15 \\
\hline JSA & 2 & 3 & 4 \\
\hline Incapacity & 5 & 7 & 7 \\
\hline Lone parents, count & 16 & 1733 & 1311974 \\
\hline Fuel poverty & 14 & & \\
\hline Pension credit, count & 45 & 4840 & 2287930 \\
\hline Occupations & & & \\
\hline Administrative & 14 & 13 & 13 \\
\hline Skilled trades & 13 & 14 & 12 \\
\hline Managers and senior officials & 24 & 17 & 15 \\
\hline Health & & & \\
\hline Good & 65 & 65 & 68 \\
\hline Fairly good & 27 & 25 & 22 \\
\hline Not good & 8 & 9 & 9 \\
\hline Long-term illness, working age & 15 & 14 & 13 \\
\hline High level unpaid caring & 21 & 20 & 20 \\
\hline Education & & & \\
\hline Adults low/no qualifications & 36 & & \\
\hline $\begin{array}{l}\text { 5 GCSEs, grade A } \\
\text { English and Maths }\end{array}$ & $\mathrm{n} / \mathrm{a}$ & 59 & 55 \\
\hline Crime & & & \\
\hline $\begin{array}{l}\text { Stolen Motor vehicle: rank of } 18 \\
\text { wards }\end{array}$ & 4 & & \\
\hline Theft from person: rank of 18 wards & 3 & & \\
\hline & & & \\
\hline
\end{tabular}




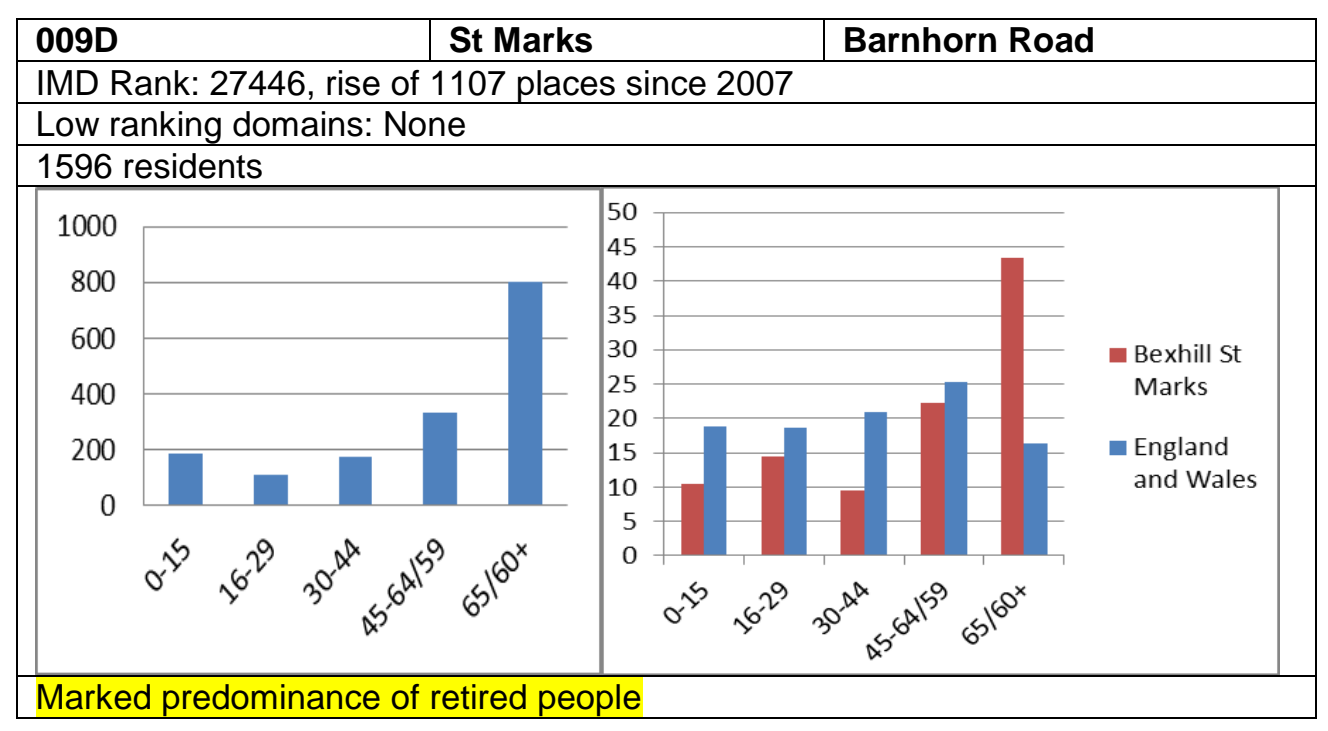

\begin{tabular}{|l|l|l|l|}
\hline & & Rother & England \\
\hline Benefits claimants August 2010 & $\%$ & $\%$ & $\%$ \\
\hline Any working age benefit & 10 & 15 & 15 \\
\hline JSA & 2 & 3 & 4 \\
\hline Incapacity & 5 & 7 & 7 \\
\hline Fuel poverty & 14 & & \\
\hline Lone Parents, count & 18 & 1733 & 1311974 \\
\hline Pension Credit, count & 45 & 4840 & 2287930 \\
\hline Occupations & & & \\
\hline Administrative & 13 & 13 & 13 \\
\hline Associated professional & 18 & 14 & 14 \\
\hline Managers and senior professionals & 20 & 17 & 15 \\
\hline Health & & & \\
\hline Good & 61 & 65 & 68 \\
\hline Fairly good & 29 & 25 & 22 \\
\hline Not good & 10 & 9 & 9 \\
\hline Working age limiting illness & 14 & 14 & 13 \\
\hline High level unpaid caring & 19 & 20 & 20 \\
\hline Education & & & \\
\hline $\begin{array}{l}\text { 5+ GCSEs A*-C, including English } \\
\text { and Maths }\end{array}$ & 67 & 59 & 55 \\
\hline Adults low/no qualifications & 37 & & \\
\hline Crime & & & \\
\hline $\begin{array}{l}\text { Stolen Motor vehicle: rank of } 18 \\
\text { wards }\end{array}$ & 4 & & \\
\hline Theft from person: rank of 18 wards & 3 & & \\
\hline & & & \\
\hline
\end{tabular}




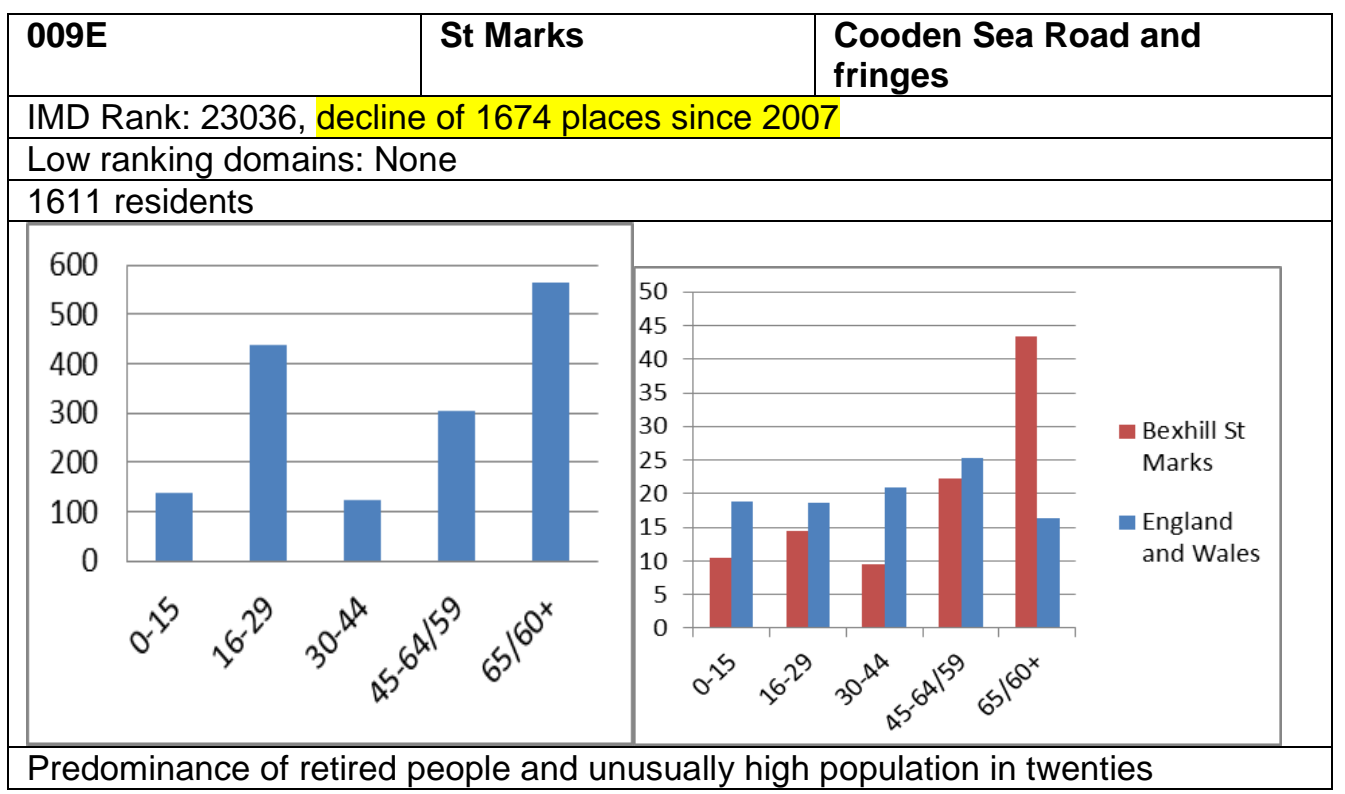

\begin{tabular}{|l|l|l|l|}
\hline & & Rother & England \\
\hline Benefits claimants August 2010 & $\%$ & $\%$ & $\%$ \\
\hline Any working age benefit & 7 & 15 & 15 \\
\hline JSA & 1 & 3 & 4 \\
\hline Incapacity & 3 & 7 & 7 \\
\hline Fuel poverty (households) & 20 & & \\
\hline Lone parents, count & 18 & 1733 & 1311974 \\
\hline Pension Credit & 55 & 4840 & 2287930 \\
\hline Occupations & & & \\
\hline Professional & 14 & 10 & 11 \\
\hline Administrative & 14 & 14 & 14 \\
\hline Managers and senior officials & 21 & 17 & 15 \\
\hline Health & & & \\
\hline Good & 70 & 65 & 68 \\
\hline Fairly good & 20 & 25 & 22 \\
\hline Not good & 10 & 9 & 9 \\
\hline Working age limiting illness & 9 & 14 & 13 \\
\hline High level unpaid caring & 16 & 20 & 20 \\
\hline Education & & & \\
\hline $\begin{array}{l}\text { 5+ GCSEs A } \\
\text { Maths } \text {-C, including English and }\end{array}$ & 69 & 59 & 55 \\
\hline Adults low/no qualifications & 31 & & \\
\hline Crime & & & \\
\hline Stolen Motor vehicle: rank of 18 wards & 4 & & \\
\hline Theft from person: rank of 18 wards & 3 & & \\
\hline
\end{tabular}




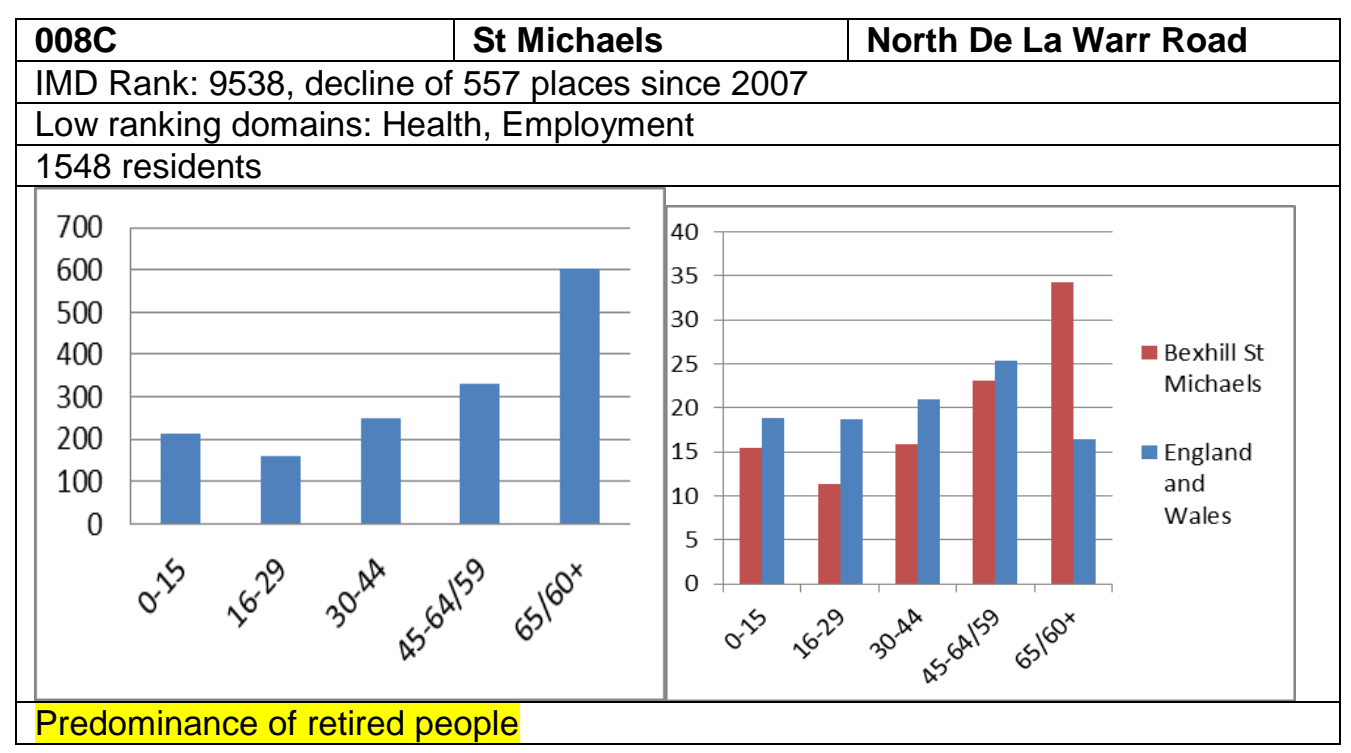

\begin{tabular}{|l|l|l|l|}
\hline & & Rother & England \\
\hline Benefits claimants August 2010 & $\%$ & $\%$ & $\%$ \\
\hline Any working age benefit & 24 & 15 & 15 \\
\hline JSA & 3 & 3 & 4 \\
\hline Incapacity & 15 & 7 & 7 \\
\hline Fuel poverty (households) & 18 & & \\
\hline Lone Parents, count & 23 & 1733 & 1311974 \\
\hline Pension Credit & 125 & 4840 & 2287930 \\
\hline Occupations & & & \\
\hline Associated professionals & 12 & 14 & 14 \\
\hline Administrative & 14 & 13 & 13 \\
\hline Managers and senior officials & 17 & 17 & 15 \\
\hline Health & & & \\
\hline Good & 61 & 65 & 68 \\
\hline Fairly good & 25 & 25 & 22 \\
\hline Not good & 15 & 9 & 9 \\
\hline Working age limiting illness & 21 & 14 & 13 \\
\hline High level unpaid caring & 27 & 20 & 20 \\
\hline Education & & & \\
\hline $\begin{array}{l}\text { 5+ GCSEs A*-C, including English } \\
\text { and Maths }\end{array}$ & 50 & 59 & 55 \\
\hline Adults low/no qualifications & 44 & & \\
\hline Crime & & & \\
\hline No areas of concern & & & \\
\hline & & & \\
\hline
\end{tabular}




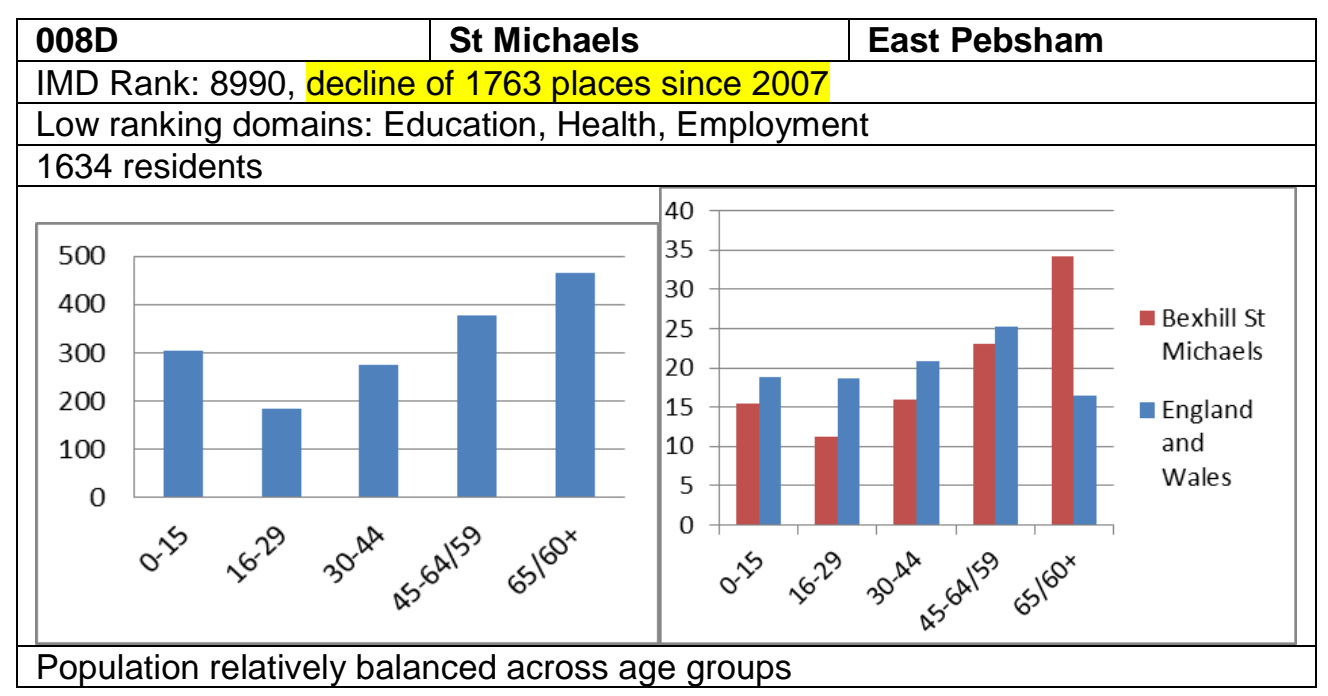

\begin{tabular}{|l|l|l|l|}
\hline & & Rother & England \\
\hline Benefits claimants August 2010 & $\%$ & $\%$ & $\%$ \\
\hline Any working age benefit & 25 & 15 & 15 \\
\hline JSA & 5 & 3 & 4 \\
\hline Incapacity & 11 & 7 & 7 \\
\hline Fuel poverty (households) & 13 & & \\
\hline Lone parents, count & 51 & 1733 & 1311974 \\
\hline Pension Credit & 90 & 4840 & 2287930 \\
\hline Occupations & & & \\
\hline Skilled trades & 14 & 14 & 12 \\
\hline Elementary occupations & 18 & 11 & 12 \\
\hline Managers and senior officials & 14 & 17 & 15 \\
\hline Health & & & \\
\hline Good & 62 & 65 & 68 \\
\hline Fairly good & 27 & 25 & 22 \\
\hline Not good & 12 & 9 & 9 \\
\hline Working age limiting illness & 18 & 14 & 13 \\
\hline High level unpaid caring & 34 & 20 & 20 \\
\hline Education & & & \\
\hline $\begin{array}{l}\text { 5+ GCSEs A*-C, including English and } \\
\text { Maths }\end{array}$ & 33 & 59 & 55 \\
\hline Adults low/no qualifications & 53 & & \\
\hline Crime & & & \\
\hline No areas of concern & & & \\
\hline
\end{tabular}




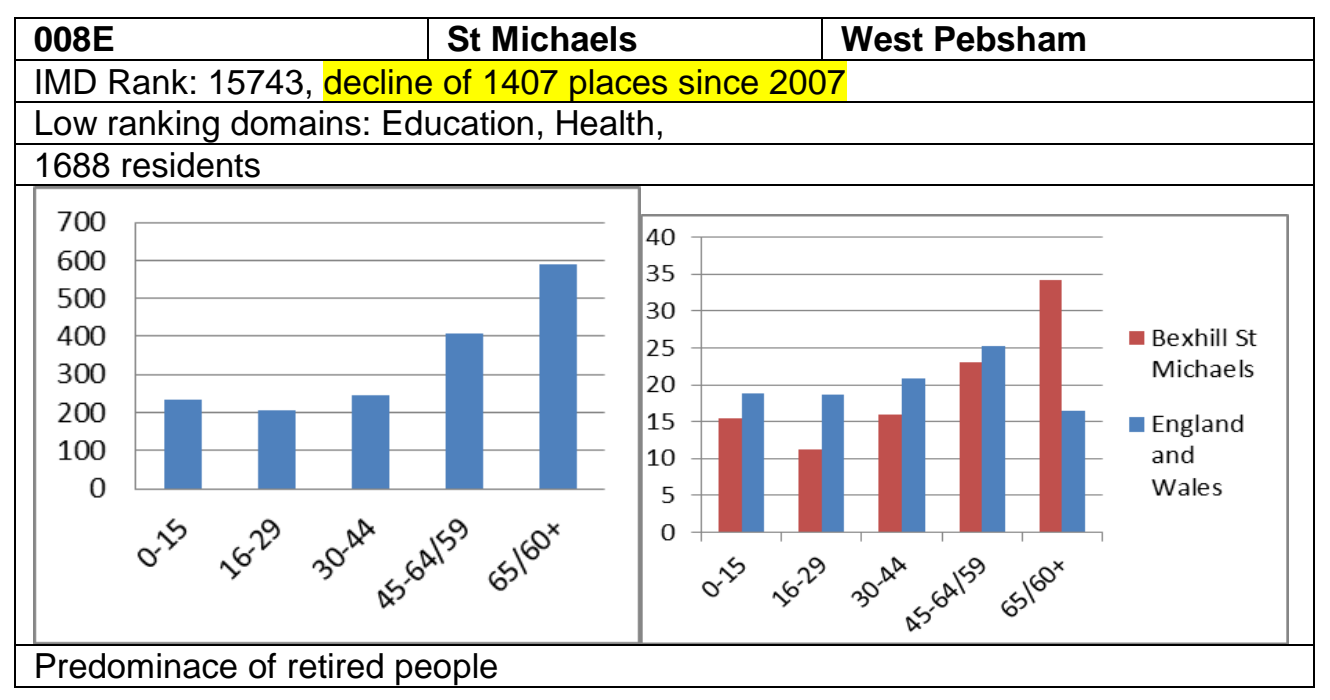

\begin{tabular}{|l|l|l|l|}
\hline & & Rother & England \\
\hline Benefits claimants August 2010 & $\%$ & $\%$ & $\%$ \\
\hline Any working age benefit & 17 & 15 & 15 \\
\hline JSA & 3 & 3 & 4 \\
\hline Incapacity & 8 & 7 & 7 \\
\hline Fuel poverty (households) & 14 & & \\
\hline Lone parents, count & 29 & 1733 & 1311974 \\
\hline Pension credit, count & 80 & 4840 & 2287930 \\
\hline Occupations & & & \\
\hline Administrative & 16 & 13 & 13 \\
\hline Skilled trades & 15 & 14 & 11 \\
\hline Associated professionals & 13 & 14 & 14 \\
\hline Health & & & \\
\hline Good & 61 & 65 & 68 \\
\hline Fairly good & 28 & 25 & 22 \\
\hline Not good & 11 & 9 & 9 \\
\hline Working age limiting illness & 14 & 14 & 13 \\
\hline High level unpaid caring & 26 & 20 & 20 \\
\hline Education & & & \\
\hline $\begin{array}{l}\text { 5+ GCSEs A*-C, including English } \\
\text { and Maths }\end{array}$ & 47 & 59 & 55 \\
\hline Adults low/no qualifications & 45 & & \\
\hline Crime & & & \\
\hline No areas of concern & & & \\
\hline & & & \\
\hline
\end{tabular}




\section{Appendix 23}

\section{Rother Indices of Multiple Deprivation Data 2010}
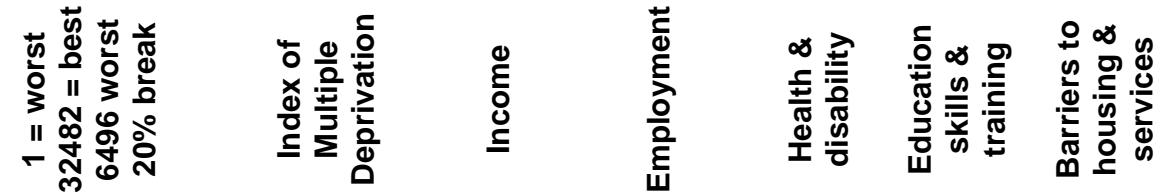

튼

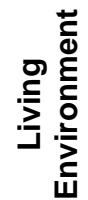

\section{Battle Town}

Rother 006A

Rother 006B

Rother 006C

Brede Valley

Rother 005A

Rother 005B

Rother 005C

Bexhill

Central

Rother 011A

Rother 011B

Rother 011C

Bexhill

Collington

Rother 009A

Rother 010A

Rother 009B

Crowhurst

Rother 006D

Rother 006E

Darwell

Rother 003A

Rother 003B

Rother 003C

27439

11026

11134

1006

18826

13511

16470

5309

2245720493

26907

23230

21054

19956

8549

8264

27755

24245

$16181 \quad 19285$

$14589 \quad 20279$

1868

18035

23015

13061

24172

15244

$15459 \quad 1252$

18842

18156

26065

808

405

26270

13062

14348

18026

11013

10208

27333

16791

21291

$8035^{-} 9336$

$6552 \quad 6$

$6019 \quad 10572$

18123

13769

3446

$7409 \quad 10194$

4764

7316

10666

15237

8375

4313

4600

7042

2367

$2827 \quad 7745$

18807

9198

4330

Eastern

Rother

Rother 004A

Rother 004B

Rother 002A

Ewhurst and

Sedlescombe

Rother 003D

Rother 003E

Bexhill

Kewhurst

Rother 010B

Rother 010C

Rother 010D

$22106 \quad 26018$

12365

1885420053

17505

14121

12237

25949

8498

$27295 \quad 30367$

20963

20404

25314

10262

31491

31453

$12364 \quad 18448$

16809

20007

4252

9882

30592

16677

$\begin{array}{ll}12364 & 18448 \\ 17030 & 20600\end{array}$

22346

18624

25107

70

(3)

31891

$20424 \quad 18035$

$\begin{array}{ll}20424 & 18035 \\ 16854 & 26287\end{array}$

18704

176

16538

17652

26038

943

19886

2067

1551

18235

2084

18322

23780

13601

23593

10802

Marsham

Rother 005D

Rother 005E

Bexhill Old

Town

Rother 007A

Rother 008A

Rother 007B

Rother

Levels

$12922 \quad 12259$

$8368 \quad 13050$

14747

$15887 \quad 12529$

$13415 \quad 14458 \quad 5094$

756

28337

11054

692

10780

1201

15618

7963

$17388 \quad 22040$

23150

19334

25502

1168

17094

20972

27171

1393

12046

18708

19062

31807

23374

$19516 \quad 18725$

12277

$12726 \quad 21014$

5719

31981

29541

$16951 \quad 16061$

13598

$\begin{array}{ll}17155 & 24889 \\ 14422 & 16487\end{array}$

12116

27610

15660

$18158 \quad 22393$

2013

$18426 \quad 18568$

208

25883

17028

$17483 \quad 22998$

$15318 \quad 17606 \quad 15844$

2055

27465

27756

$8614 \quad 8220$

626

518

23726

22342

20684

14088

12860

710

16547

19909

$14010 \quad 11194$

14143

12165

16517

29563

27546

8931

19425

24797

13525 


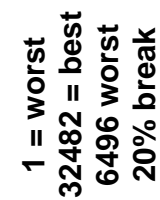

Rother 002B

Rother 002C

Rother 002D

Rye

Rother 004C

Rother 004D

Rother 004E

Bexhill

Sackville

Rother 008B

Rother 011D

Rother 011E

Bexhill St

Marks

Rother $009 \mathrm{C}$

Rother 009D

Rother 009E

Bexhill St

Michaels

Rother 008C

Rother 008D

Rother 008E

Bexhill St

Stephens

Rother 009F

Rother 010E

Rother 007C

Salehurst

Rother 001A

Rother 001B

Rother 001C

Bexhill

\section{Sidley}

Rother 007D

Rother 007E

Rother 007F

Rother 009G

Ticehurst

and

Etchingham

Rother 001D

Rother 001E

Rother 001F

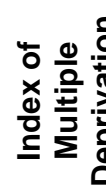

27657

15994

11510

15496

4669

13405

11618

5980

\section{8}

27446

23036

25038

26030

23402

$9538 \quad 11773$

$8990 \quad 9600$

$15743 \quad 14807$

$22490 \quad 21581$

$18117 \quad 18566$

$8467 \quad 5854$

$26167 \quad 24827$

$14357 \quad 13537$

$18346 \quad 17374$

3126

2477
691
5293

1259

7865

10162

$2477 \quad 3046$

$691 \quad 1349$

6020

12269

7342

4397

6598

13891

15804

12758

6823

25557

15731

25134

$$
17717
$$

16093

18284
17717

100607740

$24842 \quad 25259$

$17946 \quad 13657$

$22964 \quad 13125$

-

$\begin{array}{rrrrr}3947 & 2316 & 24726 & 4373 & 9814 \\ 1426 & 2469 & 20319 & 7314 & 8136 \\ 10075 & 4188 & 19307 & 15728 & 20181 \\ 5904 & 10632 & 6521 & 21777 & 21329\end{array}$

$\begin{array}{lllll}17961 & 12057 & 20898 & 18463 & 14102\end{array}$

11443

2400929676

20898

18463

14102

25760
$24915 \quad 29502$

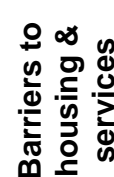

$\stackrel{Ð}{\frac{\Xi}{5}}$

:

$\begin{array}{rrr}4123 & 25354 & 21523 \\ 10596 & 29054 & 13649 \\ 6871 & 21694 & 13060\end{array}$

$\begin{array}{lll}10120 & 4367 & 5770\end{array}$

$\begin{array}{lll}12310 & 8462 & 5800\end{array}$

$\begin{array}{lll}16018 & 18207 & 12387\end{array}$

$23682 \quad 29541$

$\begin{array}{rrr}5209 & 18686 & 24824 \\ 14573 & 14100 & 10596 \\ 16055 & 21382 & 5813\end{array}$

$\begin{array}{rrr}20521 & 31730 & 29027 \\ 27897 & 31731 & 27227 \\ 4479 & 28371 & 23092\end{array}$

$\begin{array}{lll}13896 & 24626 & 17339 \\ 16390 & 21686 & 21828 \\ 12259 & 27206 & 28248\end{array}$

$\begin{array}{lrr}11172 & 28370 & 29644 \\ 22515 & 17357 & 15223 \\ 14816 & 13308 & 15177 \\ & - & - \\ 6101 & 30661 & 24684 \\ 6270 & 17111 & 11392 \\ 4151 & 28715 & 11846\end{array}$




\section{Appendix 24}

\section{Summary of Participant Career and Income Profiles}

\begin{tabular}{|c|c|c|c|}
\hline Ref & Age & $\begin{array}{l}\text { Work/retired } \\
\text { (nature of career) }\end{array}$ & $\begin{array}{l}\text { Household } \\
\text { Income/an }\end{array}$ \\
\hline \multicolumn{4}{|l|}{$\mathbf{a}$} \\
\hline $103 a$ & $55(f)$ & $\begin{array}{l}\text { Unemployed actively seeking work (worked in Public } \\
\text { Administration) } \\
\text { husband retired }\end{array}$ & $<£ 15 k$ \\
\hline $104 a$ & $51(\mathrm{~m})$ & $\begin{array}{l}\text { Gatwick airport (worked in Private sector airports: } \\
\text { supervisor) } \\
\text { wife lives in Spain }\end{array}$ & $£ 25-50 k$ \\
\hline $106 a$ & $52(\mathrm{~m})$ & $\begin{array}{l}\text { Retired - ill health (Bank Manager - Banking career) } \\
\text { single }\end{array}$ & £15-25k \\
\hline $116 a$ & $53(\mathrm{~m})$ & $\begin{array}{l}\text { Self-employed (p/t university lecturer, playwright, } \\
\text { consultant) } \\
\text { (Lecturer in Higher Education) } \\
\text { wife university lecturer }\end{array}$ & $£ 25-50 k$ \\
\hline $121 a$ & $55(\mathrm{~m})$ & $\begin{array}{l}\text { Semi-retired first aid consultant (worked in policing - Met } \\
\text { police) } \\
\text { wife housewife }\end{array}$ & $£ 25-50 k$ \\
\hline \multicolumn{4}{|l|}{ b } \\
\hline $102 b$ & $59(f)$ & $\begin{array}{l}\text { Working (Finance Officer) (Private and Public) } \\
\text { husband retired }\end{array}$ & $£ 25-50 k$ \\
\hline $113 b$ & $60(f)$ & $\begin{array}{l}\text { Semi-retired ill health. Now Health Consultant (worked } n \\
\text { Public sector - NHS nurse) } \\
\text { husband ill health retirement }\end{array}$ & $£ 15-25 k$ \\
\hline $114 b$ & 58 (f) & $\begin{array}{l}\text { Retired (worked as local govt Librarian) } \\
\text { husband retired }\end{array}$ & $£ 25-50 k$ \\
\hline $115 b$ & $59(\mathrm{~m})$ & $\begin{array}{l}\text { Unemployed (Surveyor in Private and Public sector) } \\
\text { single }\end{array}$ & $<£ 15 k$ \\
\hline $119 b$ & $\begin{array}{l}56(f) \\
\text { (Dis) }\end{array}$ & $\begin{array}{l}\text { Retired (disabled) (worked in Public sector - NHS nurse) } \\
\text { partner retired }\end{array}$ & $£ 25-50 k$ \\
\hline \multicolumn{4}{|c|}{${ }^{2}$} \\
\hline $101 \mathrm{c}$ & $65(f)$ & $\begin{array}{l}\text { Retired (worked as Bookkeeper - predominantly private } \\
\text { sector) } \\
\text { partner disabled }\end{array}$ & $£ 25-50 \mathrm{k}$ \\
\hline $109 \mathrm{c}$ & $63(\mathrm{~m})$ & $\begin{array}{l}\text { Retired (worked career in Banking) } \\
\text { single divorced }\end{array}$ & $£ 25-50 k$ \\
\hline $110 \mathrm{c}$ & $63(\mathrm{~m})$ & $\begin{array}{l}\text { Retired (Met police) } \\
\text { wife housewife }\end{array}$ & $£ 25-50 k$ \\
\hline $112 \mathrm{c}$ & $61(\mathrm{~m})$ & $\begin{array}{l}\text { Semi-retired (Private sector - Manufacturing) } \\
\text { wife retired }\end{array}$ & $>£ 50 k$ \\
\hline $118 \mathrm{c}$ & 65 (f) & $\begin{array}{l}\text { Retired (PA London - Private sector) } \\
\text { husband retired }\end{array}$ & $£ 25-50 k$ \\
\hline $120 \mathrm{c}$ & $\begin{array}{l}62(f) \\
\text { (Dis) }\end{array}$ & $\begin{array}{l}\text { Retired ill-health (Public sector - NHS administration) } \\
\text { single divorced }\end{array}$ & $<£ 15 k$ \\
\hline \multicolumn{4}{|c|}{ 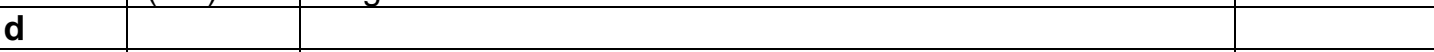 } \\
\hline $105 d$ & $70(\mathrm{~m})$ & $\begin{array}{l}\text { Retired (owner of company) } \\
\text { widowed }\end{array}$ & $£ 25-50 k$ \\
\hline $107 d$ & 69 (f) & $\begin{array}{l}\text { Retired (Public sector - NHS nursing) } \\
\text { single divorced }\end{array}$ & $<£ 15 k$ \\
\hline $108 d$ & 68 (f) & $\begin{array}{l}\text { Retired (Administration - Public sector) } \\
\text { single divorced }\end{array}$ & $<£ 15 k$ \\
\hline $111 d$ & $67(f)$ & $\begin{array}{l}\text { Retired (Secretary - Public sector) } \\
\text { Partner retired }\end{array}$ & $£ 25-50 k$ \\
\hline $117 d$ & 69 (f) & $\begin{array}{l}\text { Retired (University Librarian - Higher Education) } \\
\text { re-married, husband retired }\end{array}$ & $£ 15-25 k$ \\
\hline
\end{tabular}

